

\section{DISCLAIMER}

This report was prepared as an account of work sponsored by an agency of the United States Government. Neither the United States Government nor any agency Thereof, nor any of their employees, makes any warranty, express or implied, or assumes any legal liability or responsibility for the accuracy, completeness, or usefulness of any information, apparatus, product, or process disclosed, or represents that its use would not infringe privately owned rights. Reference herein to any specific commercial product, process, or service by trade name, trademark, manufacturer, or otherwise does not necessarily constitute or imply its endorsement, recommendation, or favoring by the United States Government or any agency thereof. The views and opinions of authors expressed herein do not necessarily state or reflect those of the United States Government or any agency thereof. 


\section{DISCLAIMER}

Portions of this document may be illegible in electronic image products. Images are produced from the best available original document. 


\section{DO MOROFIA? SOR}

This report has been reproduced directly from the best available copy.

Available from the National Technical Information Service, U. S. Department of Commerce, Springfield. Virginia 22161.

Price: Printed Copy A09

Microfiche A01

Codes are used for pricing all publications. The code is determined by the number of pages in the publication. Information pertaining to the pricing codes can be found in the current issues of the following publications, which are generally available in most libraries: Energy Research Abstracts, (ERA): Government Reports Announcements and Index (GRA and I); Scientific and Technical Abstract Reports (STAR); and publication, NTIS.PR-360 available from (NTIS) at the above address. 
DATE: DEC 3 i 109 s

DE87 004572

AEPLY TO NE-12

sUbuect: Technical Reference Book and Phase VIII Update (1986) Report for the Energy Economic Data Base Program

To: Distribution

We are pleased to send you for your information one copy each of the "Phase VIII Update (1986) Report for the Energy Economic Data Base Program" (DOE/NE-0051/3) and the "Technical Reference Book" (DOE/NE-0059/2). The program is sponsored and administered at the Department of Energy by the Planning and Analysis Group of the Office of Nuclear Energy.

The first volume is a review of the 1986 update of detailed reference powerplant cost estimates. The second provides the current technical design bases for each technical data model (of an electric generating plant) in the eighth update. It contains a set of detailed system design descriptions (supplemented with engineering drawings) for the technical data models.

This distribution is the latest in a series published since 1978. The overall program purpose is to provide periodically updated, detailed base construction cost estimates for large nuclear electric operating plants. These data, which are representative of current U.S. powerplant construction cost experience, are a useful contribution to program planning by the Office of the Assistant Secretary for Nuclear Energy.

The eighth update incorporates the results of a comprehensive update of the technical and cost information for the pressurized water reactor (PWR), large scale prototype breeder reactor nuclear powerplant (LSPB), and 488 Mwe high sulfur, coal-fired powerplant (HS5) data models. During the Phase VIII update, the LSPB, which was first incorporated into the previous update, was brought into full conformance with EEDB ground rules, and the level of detail of the data models was extended to the EEDB fully detailed level. We remind the user that the LSPB must still be considered a secondof-a-kind, pre-commercial unit, and any comparisons of it with other EEDB data models should be carefully made recognizing dissimilarity in achievement of design and cost maturity, particularly for the nuclear steam supply system and other equipment.

We welcome any comments or suggestions, and if you have any questions, please cal1 B. H. Shapiro (301-353-4874 or FTS 233-4874). Additional copies should be requested from the Office of Scientific and Technical Information, P.0. Box 62, Oak Ridge, TN 37831.
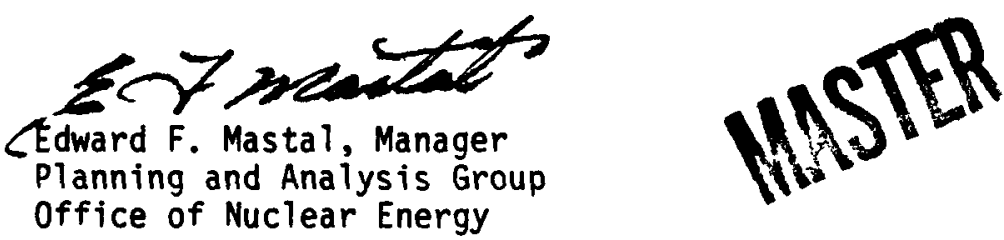


\section{Phase VIII Update (1986) Report For The Energy Economic Data Base Program EEDB - VIII}

Published: December 1986

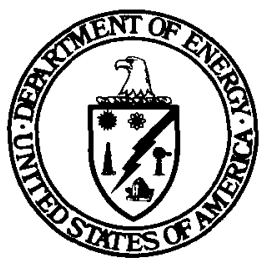

Prepared for:

U.S. Department of Energy

Assistant Secretary for Nuclear Energy

Office of Program Support

Plans and Evaulation Division

Washington, D.C. 20545

Prepared by:

United Engineers \& Constructors Inc.

Philadelphia, PA 19101

Under the Direction of

Oak Ridge National Laboratory

Under Contract No.: DE-ACO5-84OR21400 
TABLE OF CONTENTS

ENERGY ECONOMIC DATA BASE (EEDB) PROGRAM

PHASE VIII UPDATE (1986) REPORT

$\begin{array}{ll}\text { Legal Notice } & \text { i } \\ \text { List of Principal Contributors } & \text { ii } \\ \text { List of Tables } & \text { iii } \\ \text { List of Figures } & \text { vi }\end{array}$

Section

Title

Page

ES Executive Summary of the Energy Economic Data Base

ES-1

(EEDB) Program Phase VIII Update (1986) Report

Description of the Energy Economic Data Base Program

ES-1

Summary of the Phase VIII Update Technical Changes

ES-4

Results of the Phase VIII Update Cost Changes

ES-6

Implications of the Results of the Phase VIII Update ES-8

Prospects for the Future

ES-9

1.0 Overview of the Phase VIII Update Report 1-1

1.1 EEDB Program Background 1-1

1.2 The Phase VIII EEDB Program Objectives, Goals and Scope 1-3

1.3 Summary of Phase VIII Update Activities $1-4$

1.4 Presentation of the EEDB Program Report 1-5

1.5 Organization of the Phase VIII Update Report 1-7

2.0 Summary of the Phase VIII Technical/Cost Update Results 2-1

2.1 Technical Summary 2-1

2.2 Cost Summary 2-1

3.0 Causes and Effects of the Phase VIII Update Changes 3-1

3.1 Introduction 3-1

3.2 Perception of Nuclear/Coal Cost Trends 3-1

3.3 Results of the Phase VIII Update 3-2

3.4 Base Construction Cost Trends Between 1978 and 1986 3-5

4.0 Technical Update for Single Unit Nuclear and Comparison 4-1 Power Plants

4.1 Technical Data Model Update Procedure 4-1

4.2 Technical Summary 4-2

4.3 Technical Update Results 4-2

4.4 Detailed Technical Changes 4-7 
TABLE OF CONTENTS

ENERGY ECONOMIC DATA BASE (EEDB) PROGRAM

PHASE VIII UPDATE (1986) REPORT

Section

Title

$\underline{\text { Page }}$

5.0 Cost Update for Single Unit Nuclear and Comparison

5-1

Power Plants

5.1 Cost Data Model Update Procedure

5.2 Cost Summary

5.3 Detailed Costs

5.4 Cost Changes

5.5 Basis for Cost Comparisons for the PWR MEE, the PWR $\cdot B E$, the LSPB and the HS5

6.0 Review of Advanced LWR Concepts for the 1990's and Beyond 6-1

6.1 Introduction 6-1

6.2 Potential Design Features and Practices for Future 6-1 Nuclear Power Plants

6.3 U.S. Advanced LWR Program 6-2

6.4 Advanced LWR Nuclear Power Plant Technical Design 6-3 Features

6.5 Advanced LWR Nuclear Power Plant Potential Cost and 6-5 Schedules

7.0 References, Definitions and Glossary 7-1

7.1 References and Bibliography 7-1

7.2 Definitions 7-3

7.3 Glossary of Acronyms and Abbreviations 7-5

\section{Appendix}

A-1

Energy Economic Data Base (EEDB) Program - Bibliography of Potential Design Features/Practices for Nuclear

$A-1-1$

Power Plants of the 1990's and Beyond

A1.1 Bibliography I (Alphabetica1)

A-1-3

Al.2 Bibliography II (Topical)

$A-1-6$ 
LEGAL NOTICE

ENERGY ECONOMIC DATA BASE (EEDB) PROGRAM

PHASE VIII UPDATE (1986) REPORT

This report was prepared under the funding of the U.S. Department of Energy through Oak Ridge National Laboratory Subcontract 43X-86004V. Neither the United States Government or any agency thereof, nor any person acting on behalf of the United States Government:

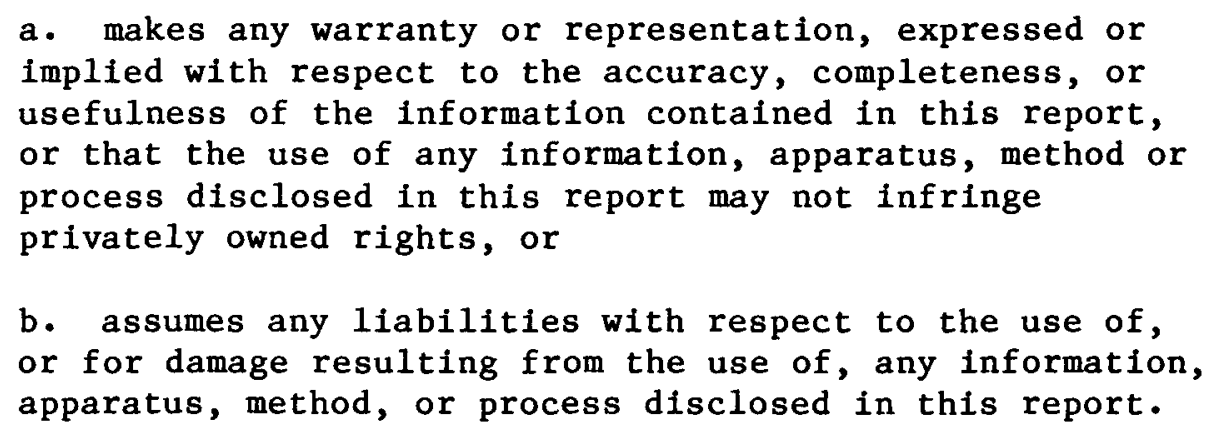

As used in the above "person acting on behalf of the United States Government" includes any employee or contractor thereof or any employee of such contractor to the extent that such employee prepares, disseminates, or provides access to, any information pursuant to his employment or contract with the United States Government, or his employment with such contractor. 
LIST OF PRINCIPAL CONTRIBUTORS

ENERGY ECONOMIC DATA BASE (EEDB) PROGRAM

PHASE VIII UPDATE (1986) REPORT

J. H. Crowley, Manager Advanced Engineering Department

*R. E. Allen, Program Project Manager Energy Economic Data Base Program

R. G. Benedict

M. S. Cooper

R. J. Gramatges

A. J. Karalis

*B. J. Menaker

*D. 0 . Nicodemus

$*_{H}$. E. Painter

J. J . Wolf

E. J. Ziegler

J. F. Smith

‡ Princlpal Authors 


\section{LIST OF TABLES}

ENERGY ECONOMIC DATA BASE (EEDB) PROGRAM

PHASE VIII UPDATE (1986) REPORT

Table

Title

Page

Number

Nuclear and Coal-Fired Power Plant Cost Experience

Number

ES-1

Summary of the Phase VIII Update (1986)

ES-11

ES-2

Base Construction Costs

ES-3

Comparison of Phase I (1978) and Phase VIII (1986)

ES-13

Updates - Base Construction Costs

ES-4

Phase I (1978) to Phase VIII (1986) Update

ES-14

Base Construction Cost Increases for the

$\mathrm{PWR} \cdot \mathrm{ME}, \mathrm{PWR} \cdot \mathrm{BE}$ and $\mathrm{HS} 5$

ES-5

Comparison of Phase I (1978) and Phase VIII (1986)

ES-15

Updates - PWR/HS5 Base Construction Cost Ratios

ES-6

LWR Plant Designs for the Future (LWR $\cdot F$ ) -

ES-16

Potential Schedule and Cost

$1-1$

Technical Data Model Update History -

Nuclear Power Generating Stations (Sheet 1)

$1-8$

Comparison Power Generating Stations (Sheet 3)

Notes (Sheet 4)

$1-2$

Nuclear and Comparison Power Generating Stations

$1-12$

for the Phase VIII Update (1986)

$1-3$

EEDB Phase VIII Update (1986) Technical Reference

$1-13$

Book - Table of Contents - Table List - Figure List

2-1 Nuclear Power Generating Stations -

$2-2$

Technical Data Models Base Parameter Summary

$2-2$

Comparison Power Generating Station -

$2-16$

Technical Data Model Base Parameter Summary

$2-3$

Base Construction Cost Update Summary ( $\$ 1986)$

$2-23$

$2-4$

Normalized (1144 MWe) Base Construction Cost

$2-24$

Update Summary ( $\$ 1986)$

$2-5$

Normalized ( 3800 MWt) Base Construction Cost

$2-25$

Update Summary ( $\$ 1986)$

4-1 Direct Commodity/Equipment/Labor Summary for

$4-18$

Nuclear and Comparison Power Generating Stations

for 1986 


\author{
LIST OF TABLES (cont 'd) \\ ENERGY ECONOMIC DATA BASE (EEDB) PROGRAM \\ PHASE VIII UPDATE (1986) REPORT
}

Table

Title

Page

Number

4-2

Direct Commodity/ Equipment/Labor Summary for

Number

Nuclear and Comparison Power Generating Stations for 1984

4-3

Direct Commodity and Craft Manhour Summary -

$4-20$ 1144 MWe Pressurized Water Reactor Nuclear Power Generating Station - Median Experience (PWR·ME)

4-4 Direct Commodity and Craft Manhour Summary 1144 MWe Pressurized Water Reactor Nuclear Power Generating Station - Best Experience (PWR・BE)

4-5 Direct Commodity and Craft Manhour Summary 1311 MWe Large Scale Prototype Breeder Nuclear Power Generating Station (LSPB)

$4-6$

Direct Commodity and Craft Manhour Summary 488 MWe High Sulfur Coa1-Fired Power Generating Station (HS5)

$4-7$

Division I Regulatory Guides Issued or Revised Between January 1, 1984 and January 1, 1986 and Their Projected Impact on EEDB Phase VIII Update Technical Features and Cost Estimates

Cooling Methods Utilized in U.S. Nuclear Power

$4-27$ Plants (Two Sheets)

Natural Draft vs. Round Mechanical Draft Cooling Towers for the PWR on the Middletown Site

$5-1$

Major Equipment Estimating Quotations Received for the PWR, LSPB and HS5 and Major Equipment Estimates Based on Estimating Quotations Received During the Phase VII Update

5-2 Preferential and Discretionary Items Excluded from EEDB Base Construction Costs

5-3

1144 MWe Pressurized Water Reactor NPGS - Median Experience (PWR・ME) - Base Construction Cost Estimate 
LIST OF TABLES (cont' $d$ )

ENERGY ECONOMIC DATA BASE (EEDB) PROGRAM

PHASE VIII UPDATE (1986) REPORT

Table

Number

Title

$$
5-5
$$

1311 MWe Large Scale Prototype Breeder NPGS

(LSPB) - Base Construction Cost Estimate

$5-6$

488 MWe High Sulfur Coal FPGS (HS5) -

$5-17$

Base Construction Cost Estimate

$5-7$

Phase VII to Phase VIII Update Base Construction Cost Increases for the Pressurized Water Reactor Nuclear Power Generating Station - Median

Experience (PWR $\cdot M E)$

$5-8$

Phase VII to Phase VIII Update Base Construction

$5-19$

Cost Increases for the Pressurized Water Reactor

Nuclear Power Generating Station - Best

Experience $(\mathrm{PWR} \cdot \mathrm{BE})$

5-9

Phase VII to Phase VIII Update Base Construction

Cost Increases for the 488 MWe High Sulfur CoalFired Power Generating Station (HS5)

Contents of Commodity/Equipment/Labor Accounts

in Tables 5-7, 5-8 and 5-9

Comparison of the PWR $\cdot \mathrm{ME}, \mathrm{PWR} \cdot \mathrm{BE}, \mathrm{LSPB}$ and HS5

Factory Equipment Plus Site Material Costs

Comparison of the $\mathrm{PWR} \cdot \mathrm{ME}, \mathrm{PWR} \cdot \mathrm{BE}, \mathrm{LSPB}$ and HS 5

NSSS Advanced Design Feature Objectives for the

6-2

BOP Advanced Design Feature Objectives for the LWR $\cdot F$

$6-8$

Potentlal Construction Schedules for the LWR $F$

6-9 As Compared with the EEDB PWR BE and the EEDB HS 5

6-4

LWR $\cdot \mathrm{F} / \mathrm{PWR} \cdot \mathrm{BE} / \mathrm{HS} 5$ Base Construction Cost Comparison

$6-10$ 


\section{LIST OF FIGURES}

ENERGY ECONOMIC DATA BASE (EEDB) PROGRAM

PHASE VIII UPDATE (1986) REPORT

Figure

Number

3.1

3.2

3.3
Title

Comparison of Phase I (1978), Phase VII (1984) and Phase VIII (1986) PWR and HS5 Base Construction Costs

Comparison of Phase I (1978), Phase VII (1984) and Phase VIII (1986) PWR and HS5 Factory Equipment

Plus Site Material Cost

Comparison of Phase I (1978), Phase VII (1984) and

Phase VIII (1986) PWR and HS5 Labor Cost
Page

Number

3-12

$3-13$

$3-14$ 


\author{
EXECUTIVE SUMMARY OF THE \\ ENERGY ECONOMIC DATA BASE (EEDB) PROGRAM \\ PHASE VIII UPDATE (1986) REPORT
}

DESCRIPTION OF THE ENERGY ECONOMIC DATA BASE PROGRAM

The objective of the Energy Economic Data Base (EEDB) Program is to provide current, representative and consistent power plant technical and cost information to the U.S. Department of Energy (DOE). These data are useful for program planning by the Office of the Assistant Secretary for Nuclear Energy, because they are representative of current U.S. power plant construction cost experience and are developed on a consistent, organized basis.

\title{
Historica1 Basis
}

This update of the Energy Economic Data Base is the latest in a series of technical and cost studies prepared by United Engineers \& Constructors Inc., during the last 22 years. These studies have been sponsored by the U.S. Department of Energy, the U.S. Nuclear Regulatory Commission and their predecessor organizations, the U.S. Energy Research and Development Admintstration, and the U.S. Atomic Energy Commission. The EEDB and its antecedent studies provide a common and consistent detailed basis for evaluating and comparing the economic viability of a variety of nuclear and alternative power generating stations.

The data base was assembled in 1978 and has been updated regularly since then. The purpose of the updates has been to reflect the Impact of changing regulations and technology on the costs of electric power generating stations. This Phase VIII Update (1986) Report documents the results of a comprehensive update of the technical and cost information for the pressurized water reactor nuclear power plant (PWR), large scale prototype breeder reactor nuclear power plant (LSPB) and 488 MWe high sulfur coal-fired power plant (HS5) technical and cost data models. The update provided costs for these nuclear and comparison power plants that are representative of current experience and practice. Costs for the PWR are given in terms of both the best* and median industry cost experience. Consequently, the Phase VIII Update has continued to provide important benchmark information through which technical and cost trends may be identified that have occurred since 1978 .

Power Plant Cost Trends and The EEDB Program

The EEDB updates regularly reported cost estimates for selected types of power plants based on technical developments and economic and regulatory conditions current for the reference date of each update. That is, they incorporate costs for current regulatory requirements, design, construction and management practices, labor productivity, and labor/material. Costs are developed as direct and indirect base construction costs. Direct costs are the costs of commodities, equipment and their installation labor. Indirect costs are the costs of construction sexvices, engineering, construction management, field supervision and testing. Contingency, owner's and inflation related costs are not included.

* "Best industry cost experience" is indicative of a small group of units at the low end of the cost range rather than a single specific untt. 
The Phase VI (1983) and the preceding five EEDB Updates 1dentified a continuing upward trend in light water reactor power plant (LWR) costs, relative to those of coal-fired power plants. The principal drivers behind this trend were increases caused by sharp rises in the quantities of commodities, construction (craft) labor manhours, and englneering and construction supervision manhours. Labor (craft, engineering and field supervision) costs became the predominant cost driver in the Phase VI Update. These trends have continued in the Phase VII (1984) and Phase VIII (1986) Updates, and are reflected in the median industry experience based PWR data model. On the othex hand, best industry cost experience plant designs appear to have the continued potential to remain competitive with coal-fired power plants. This potential is reflected in the best experience based PWR data model incorporated into the Phase VII Update and updated during the Phase VIII Update. Realization of the potential, however, may be dependent on the application of advanced design features currently being investigated and the taking of government and industry actions necessary to encourage deployment.

Continuing increases in nuclear power plant base construction costs are not in themselves a new development. Nevertheless, through the use of this cost driver information, the EEDB has become an important tool in identifying those cost elements that have been most susceptible to change, and the factors that have been dominant in causing their change. The EEDB may also be utilized to identify those proposed advanced features that are most effective in reducing or eliminating cost elements that have been contributing to increasing plant costs. This kind of information may provide a basis for identifying and developing corrective measures that permit nuclear energy to develop as an increasingly competitive source of electric power.

Data Base Content

The data base contains technical and base construction cost data models for 16 different types of nuclear and comparison power generating stations. The 16 power plant data models included in the data base are 1isted below. Those data models that were updated in the Phase VIII Update are marked with a "VIII." The remaining data models are marked with a number indicating the year in which they were last updated. The 1195 MWe Boiling Water Reactor Plant was updated in 1986 in a separate effort sponsored by the U.S. Nuclear Regulatory Commisston.

\section{Nuclear Power Generating Stations}

861195 MWe Boiling Water Reactor Plant (BWR)

82858 MWe High Temperature Gas Cooled Reactor Plant - Steam Cycle (HTGR-SC)

82150 MWe High Temperature Gas Cooled Reactor Plant - Process Steam (HTGR-PS)

VIII 1144 MWe Pressurized Water Reactor Plant - Median Cost Experience (PWR ME)

VIII 1144 MWe Pressurized Water Reactor Plant - Best Cost Experience (PWR·BE)

821260 MWe Pressurized Heavy Water Reactor Plant (PHWR)

831457 MWe Liquid Metal Fast Breeder Reactor Plant (LMFBR)

VIII 1311 MWe Large Scale Prototype Breeder Reactor Plant (LSPB) 


\section{Comparison Fossil Power Generating Stations}

$81 \quad 1240$ MWe High Sulfur Coal Plant (HS12)

83791 MWe High Sulfur Coal Plant (HS8)

$81 \quad 1244$ MWe Low Sulfur Coal Plant (LS12)

81795 MWe Low Sulfur Coal Plant (LS8)

81630 MWe Coal Gasification Combined Cycle Plant (CGCC)

VIII 488 MWe High Sulfur Coal Plant (HS5)

82480 MWe Low Sulfur Coal Plant (LS5)

82360 MWe Low Sulfur Coal Plant - District Heating (LS5-D/H)

In the EEDB, base construction costs are in constant dollars and contain no arbitrary factors, such as contingency or escalation. Capital costs are the sum of the base construction costs and a number of other factors, such as owner's costs, contingency, escalation and allowance for funds used during construction. Users of the data base may apply these factors to the base construction costs to develop capital costs that sutt their unique scenarios.

\section{Technica1/Cost Data Mode1s}

Each cost estimate is based upon a detailed technical data model for over 50 major structure/systems and up to 400 subsystems. Each technical data model includes system design descriptions, engineering drawings, milestone schedules and a detailed equipment 1ist. Each equipment list contains up to 1250 mini-specifications and up to 10,000 data lines of commodity, equipment and labor hour quantities and costs. The technical data models are based on actual current power plant designs and over 50 years of power plant design and construction experience. Additionally, the data models are periodically checked against actual field data to assure compatibility with current technical practice and cost experience.

Perhaps the most important attribute of the EEDB is the fact that assumptions and ground rules are clearly identified and are applied uniformly to all technical/cost updates. Site related factors are normalized by locating each technical data model on a common hypothetical "Middletown" site, for which there is a detalled geological and environmental description. Adjustments may be made to the technical/cost data models to reflect the characteristics of actual sites.

Each technical and cost data model is assembled and manipulated in accordance with a detalled and uniform code-of-accounts. Because of this code-of-accounts and the level of detall, ground rules, and periodic alignments with field data mentioned above, the users of the EEDB may have confidence that the data models are highly comparable, internally consistent and representative of current experience.

EEDB Program Estimating Approach

The EEDB estimating approach promotes understanding and acceptance of reported 
costs, because all components of "bottom-1ine" amounts in the different estimates are readily identified and compared through a uniform accounting system. For example, base construction costs are presented as equipment, 1abor, and site material costs in the uniform code-of-accounts. Each system, component or commodity that is common to two or more of the technical data models has the same account identifier in every data model. This feature, together with the high level of detail furnished, is the means by which a consistent comparison of commodities, components and manhours is accomplished among diverse a1ternatives whether they are already incorporated in the data base or an existing data model is modified to represent their unique characteristics.

All estimates are point estimates that generally represent the median of an actual or projected range of costs for each data model for the update year. Variations from update to update in the avallability and quality of power plant technical and cost data tend to obscure actual year to year cost changes. Consequently, the series of point estimates generated during the eight EEDB updates are considered to be more representative of cost trends than of point-to-point variations.

\section{SUMMARY OF THE PHASE VIII UPDATE TECHNICAL CHANGES}

\section{Major Activities}

Selection of technical/cost data models to be updated or added to the data base and related activities for a given update are based on current DOE objectives and availability of resources. During the Phase VIII Update, the following major activities were completed:

- Phase VII (1984) PWR $\cdot \mathrm{ME}, \mathrm{PWR} \cdot \mathrm{BE}$ and HS5 technical/cost data models were refined and updated to January 1, 1986 with particular emphasis on improving the technical maturity of the various accounts and particularly the main condenser heat rejection system accounts.

- The EEDB LSPB technical/cost data model, based on the EPRI/COMO (Consolidated Management office for the Liquid Metal Fast Breeder Reactor) LSPB and EEDB Program ground rules, was extended to a level of detail comparable to that of the PWR and HS5, particularly in the structural accounts.

- The EEDB Technical Reference Book was updated for the PWR, LSPB and HS 5 and expanded to include bullding/equipment arrangement drawings and design/construction milestone schedules for the LSPB.

\section{Phase VIII Technical Update Process}

It was observed during the Phase VIII Update that nuclear power plant costs continued to escalate above the general inflation rate, but that the slackening in the rate of increase, first observed during the Phase VII Update, 
continued. This observation was based on confidential 1984/1985 field data that was available during the update. Table ES-1 provides the current cost experience for large light water reactor power plants and a range of coal-fired power plants, which are under construction and over 45 percent complete. For the nuclear power plants, the predominant cost drivers remalned craft labor, engineering and field supervision manhours.

An in-depth review was performed on the PWR PEE, PWR BE and HS5 to adjust their technical data models against current construction experience, as represented by avallable field data, other appropriate studies and United Engineers' current experience. Adjustments were made to quantities of commodities, equipment and manhours for each technical data model's structures/systems at the nine-digit code-of-accounts level of detail. After these adjustments were made, the PWR and HS5 technical data models' total quantities of commodities and manhours were found to be reasonably representative of current industry experience and practice.

The PWR has been representative of median nuclear power plant cost experience in the EEDB program since 1978. Because of the increasing spread in the nuclear power plant cost range, however, it has become less representative of better nuclear power plant cost experience. During the Phase VI Update (1983), the PWR BE was created from the old EEDB PWR to support cost estimates that were more representative of commercially viable systems anticipated for the 1990's. The modified technical data models were based on the current best industry cost and manhours experience and the assumption that regulatory reforms and improved construction practices would be promulgated and implemented. The old EEDB PWR became the PWR ME and continued to be representative of median industry cost experience. During the Phase VII Update (1984), additional refinements were made to the $\mathrm{PWR} \cdot \mathrm{BE}$ to improve its representation of best nuclear power plant cost experience. During the Phase VIII Update, the PWR 'ME and PWR'BE were updated to reflect the current median or best experlence and practice. For the Phase VIII Update, the PWR $\cdot M E$ costs are on the order of 97 percent of median experience costs, and the PWR BE costs are are on the order of 90 percent of best experience costs. The differential between PWR BE and best experience costs in the Phase VIII Update represents the upward drift in best experience costs since 1983 .

A variety of technical refinements were made to the PWR $M E$, PWR $\cdot \mathrm{BE}$ and HS5 during the technical and cost review of these technical data models. This effort was supplemented with the updating of the structure/system design descriptions, building/equipment arrangement drawings and licensing/engineering/construction milestone schedules. Reviews of structure/system configuration, parameters and installation manhours were made based on these documents. In particular, the mechanical draft cooling towers in each of the technical data models were replaced with natural draft cooling towers to reflect current practice and economics for the Middletown site. This change resulted in an increase in net plant output (five MWe for the PWR and LSPB and two MWe for the HS5) and a decrease in base construction costs. 
A new liquid metal reactor technical data model was incorporated into the EEDB to replace the LMFBR during the Phase VII (1984) Update. The new data model was based on the EPRI/CoMO (Consolidated Management office for the Liquid Metal Fast Breeder Reactor) Large Scale Prototype Breeder Nuclear Power Plant (LSPB). The substitution of the LSPB for the LMFBR in the EEDB reflected the designation of the LSPB as the base liquid metal reactor concept by the U.S. Department of Energy. A major effort during the Phase VIII Update was to extend the level of detail of the LSPB data models from the EEDB summary level to the EEDB fully detailed level. During this process, the data model was also brought into full conformance with EEDB ground rules. Engineering drawings and milestone schedules were developed for the LSPB in support of this effort. Consequently, the EEDB LSPB is fully consistent and comparable with the other EEDB data models. Although the EEDB nth-of-a-kind cost parameters (installation rates, labor rates and unit costs) were incorporated into the LSPB cost data model, the LSPB must still be considered and referred to as a second-of-a-kind, precommercial unit. This differentiation is required because the EPRI/CoMO replica (second-of-a-kind) costs were used for the costs for the EEDB LSPB nuclear steam supply system and other equipment. Further analysis on an nth-of-a-kind basis is required for these items before the EEDB LSPB may be considered to be on an nth-of-a-kind basis. The PWR and HS5 represent nth-of-a-kind, commercial designs and costs.

The Phase VII Technical Reference Book was updated during the Phase VIII Update. The conceptual system design descriptions for the 50 major structures and systems for each of the nuclear and coal-fired power plants updated or extended in Phase VIII were revised to reflect the changes, modifications, extensions and refinements discussed above. Their supporting tabulations of key technical parameters, plot plans, summary heat balance diagrams, engineering drawings and milestone schedules were revised accordingly. In addition, the newly developed engineering drawings and milestone schedules for the LSPB were incorporated into the Phase VIII Technical Reference Book.

The adjustments described above comprise a major update to the EEDB technical data models. An lmportant result of the effort is that the EEDB technical features and resulting cost estimates are currently reflective of nuclear and coal-fired power plant construction experience. Consequently, the data base is available to serve as a current benchmark for identifying trends in quantities of commodities, equipment and manhours and their related costs for those types of power plants included in the update.

\section{RESULTS OF THE PHASE VIII UPDATE COST CHANGES}

\section{Summary of the Cost Results}

The Phase VII (1984) base construction costs were recalculated during the Phase VIII Update based on updated pricing information and the adjusted and revised technical data models described above. Quantities of commodities and equipment were derived from the updated technical data models. Updated equipment prices were obtained from manufacturers of major equipment or estimated based on data recelved in the Phase VII Update. Installation rates, and 
engineering and field supervision manhours were based on avallable field data and Untted Engineers' current experience. Commodity untt costs and labor rates were revised in accordance with national indices and United Engineers' project experience.

A summary of the Phase VIII Update base construction cost results is given in Table ES-2. All costs are given in January 1, 1986 constant dollars and include the consequences of safety and environmental regulations in effect on that date. The table illustrates the cost differences between median and best experience nuclear power plants and between nuclear and coal-fired power plants.

The base construction (direct + indirect) costs exclude owner's costs, contingency, fixed charges, escalation and allowance for funds used during construction. The nuclear power plant costs include the effects of design features that resulted from the Three Mile Island incident. The coal-fired power plant costs include costs for enclosed bollers and turbinegenerators, electrostatic precipitators, and wet lime flue gas desulfurization systems (scrubbers). Both nuclear and coal-fired power plants include costs for natural draft cooling towers, river water make-up, and treatment of waste water discharge. The coal-fired power plant costs include costs for coal storage pile water runoff and seepage control systems.

Summaxy of Cost Changes

An overview of the EEDB cost changes, that have occurred between the Phase I Update in 1978 and the Phase VIII Update in 1986, is given in Table ES-3. A11 of the values in Table ES-3 are given in 1986 constant dollars to remove the effects of inflation. Th1s table shows that the PWR $\cdot M E$ has experienced a significant real base construction cost increase between 1978 and 1986, relative to that experienced by the HS5. On the other hand, the table shows that the cost increase for the PWR BE is similar to that experienced by the HS5. The PWR ME has more than doubled in cost between 1978 and 1986, while the PWR $\cdot B E$ and the HS5 costs have increased by about 30 and 25 percent, respectively. Of the increase for the PWR $\cdot M E$, over 25 percent occurred between the Phase V (1982) and Phase VI (1983) Updates, while the HS5 remained relatively constant in cost between these two updates.

The cost changes for the PWR's from 1978 through 1986 have been driven by increases in commodities and manhours, particularly in the case of the PWR ME during 1982 and 1983. The cost change for the PWR $M E$ was primarily caused by the large increase in manhour costs that occurred in the Phase VI Update (1983). The cost change for the HS5 was primarily caused by the increase in costs for flue gas cleaning, waste water treatment and various technical refinements; however, the major impact was caused by a near tripling of the flue gas cleaning equipment installed costs. The HS 5 cost changes largely offset the PWR $\cdot B E$ cost changes between 1978 and 1986 even though they had different aspects. The HS5 cost changes were unable to offset the large labor cost increases in the PWR ME. In all three cases, however, the major cost increases appear to be related to changes in and interpretation of regulations. 
The LSPB was a new cost entry for the Phase VII Update and was updated in the Phase VIII Update. It is based on a second-of-a-kind replica plant that has a "best experience" class of labor costs, but has not reached the cost maturity of a commercial power plant, particularly in the area of the nuclear steam supply system. Since the other updated EEDB data models represent nth-of-akind commercial costs, comparisons between the LSPB and the PWR or HS5 in Table ES-3 must be made with care.

\section{IMPLICATIONS OF THE RESULTS OF THE PHASE VIII UPDATE}

\section{Influence of Base Construction Cost on Nuclear/Coal Cost Comparisons}

Historically, light water reactor nuclear power plants have had lower fuel costs but higher base construction costs and, therefore, higher total capital costs than coal-fired power plants. On the average, the much higher fuel costs for the coal-fired power plants have in the past been projected to more than offset the higher base construction costs of nuclear power plants. Therefore, nuclear power plants were percelved to produce electrictity at a lower cost than many coal-fired power plants over their projected life cycles.

As shown in Tables ES-3 through ES-5, the base construction costs for the median cost experience nuclear power plants have been increasing at a much faster rate than for coal-fired power plants. Consequently, these nuclear power plants may no longer be competitive with coal-fired power plants except on a marginal or individual basis.

\section{Comparison of Nuclear/Coal Cost Ratios}

The EEDB PWR and HS5 base construction cost comparison in Table ES-4 for the period between 1978 and 1986 shows a much faster rate of increase for the PWR $M E$ costs than for either the PWR BE costs or the HS5 costs. The values in this table are given in 1986 constant dollars to remove the effects of inflation from the comparison. Table ES-4 indicates that during this period the PWR $M E$ base construction costs increased at a rate of 11 percent per year above general inflation, while the PWR $\cdot \mathrm{BE}$ and the HS5 base construction costs have each increased at a rate of three percent per year above general inflation. The table also indicates that the indirect costs for each of the PWR's and the HS5 have increased at a faster rate than the direct costs; however, the PWR $\cdot \mathrm{ME}$ increase in indirect costs is much more significant. The increase in indirect costs for the PWR $M E$ is primarily a result of rapidly rising engineering and field supervision manhours.

Table ES-4 exemplifies the nuclear and coal-fired power plant cost trends implied by the results of the Phase I through Phase VIII Updates of the EEDB. The cost trends are most significant when they are used to compare the relative costs of the nuclear option (as represented by the EEDB PWR) and an alternative (as represented by the EEDB HS5). The nuclear/coal-fired power plant cost trends, as identified by the changes in EEDB cost ratios between 1978 and 1986, are given in Table ES-5. On a dollars per kilowatt basis, the PWR $M E$ costs increased from a two percent differential in 1978 to an 82 
percent differential in 1986. On the same basis, the PWR BE costs increased slightly from a two percent differential in 1978 to a four percent differential in 1986 .

\section{Cost Drivers}

As shown in Table ES-4, base construction costs for the EEDB median experience nuclear power plant have increased at a rate above general inflation which is over three times that for the comparable EEDB coal-fired power plant. The rate of increase is also three times that of the average rate of inflation over the period. As shown in Table ES-5, the median experience PWR/HS5 cost ratio has increased by four-fifths between 1978 and 1986. The increases represented in Tables ES-4 and ES-5 have been driven by increases in commodities, equipment and manhours, but more recently the increases have been in craft manhours and indirect costs. Since 1978, 1abor (craft, engineering and field supervision) costs have increased by 220 percent above general inflation for the $P W R \cdot M E, 40$ percent for the PWR ${ }^{\cdot B E}$ and 25 percent for the HS5. By contrast, commodities/equipment costs have only grown by 35,15 and 20 percent above inflation respectively for these three plants.

The fleld surveys made concurrently with the Phase $V$ and Phase VI Updates and $1984 / 1985$ field data found and verified that both regulatory and construction practices were responsible for the continuing increase in manhouxs. Engineering and engineering support services, construction crafts and indirect labor associated with temporary facilities and field supervision were primary contributors to this increase. The data also showed a correlation between high manhours and high costs as well as low manhours and low costs.

\section{PROSPECTS FOR THE FUTURE}

\section{Current Prospects}

If the cost and manhour experience of the median to high cost nuclear power plants continues, nuclear power plants will become increasingly less competitive with coal-fired power plants and other alternatives. On the other hand, if the best nuclear power plant cost and manhour experience were to prevail in the future, nuclear power plants would most likely be at least as competitive with coal-fired power plants as they were in 1978, when the EEDB Phase I Update showed a PWR/HS5 dollars per kilowatt (electrical) capital cost ratio of 1.02 .

\section{Future Prospects}

During the Phase VII and VIII Updates, a review was conducted to determine the activities under way that might improve the designs and costs for the nuclear power plants for the 1990's and beyond that might be included in future updates. A variety of organizations are working on design, maintenance and operation improvements for current plants, and advanced concepts based on current technology for future plants. Among the subjects being investigated by such organizations as DOE, EPRI, INPO, NRC and various utilities, manufacturers and architect-engineers are: 


\section{ALARA *}

Advanced Concepts

Availability

Computers

Equipment Qualification

Maintenance Activities
Modularization

Operations/Operability

Radioactive Waste Management

Robotics \& Axtificial Intelligence

Selsmic Qualification

Steam Generators and Turbine Generators

* (Radiation Exposure) As Low as Reasonably Achievable

In particular, EPRI is sponsoring the development of a requirements document for advanced light water reactor nuclear power plants (ALWR) and, in conjunction with DOE, conceptual designs for advanced small (600 MWe) 1ight water reactor nuclear power plants. The requirements document will give detailed requirements for future ALWR units that have improved and advanced design characteristics. The objective is to promote destgns that are safer, simpler, cheaper and easier to construct, maintain and operate.

An assessment of the various activities and objectives described above indicates that they will have a significant impact on cost and schedule when applied to current best industry experience designs. Results of a preliminary application of this assessment to the Phase VIII PWR BE technical/cost data are given in Table ES-6. The LWR for the future (LWR $\cdot F$ ) appears to have the potential for being more affordable than current best experience nuclear power plants and, consequently, for being cost competitive with future coalfired power plants. 
Effective Date $1 / 1 / 86$

TABLE ES-1

ENERGY ECONOMIC DATA BASE

PHASE VIII UPDATE

NUCLEAR (a) AND COAL-FIRED (b) POWER PLANT
COST EXPERIENCE

\begin{tabular}{c} 
Cost \\
Experience \\
Level \\
\hline
\end{tabular}

Best

Average

Highest

\begin{tabular}{|c|c|}
\hline NPGS & FPGS \\
\hline 1618 & 648 (d) \\
\hline 2574 & $1340(d)$ \\
\hline 3739 & 1942 \\
\hline
\end{tabular}

1942

\footnotetext{
(a) 900 MWe to 1300 MWe, Single or First-on-Site LWR Units Under Construction, but Over 65 Percent Complete

(b) 250 MWe to 850 MWe, Single or First-on-Site Coal-Fired Units, with Flue Gas Desulfurization Systems, Under Construction, but Mostly Over 45 Percent Complete

(c) Base Construction Cost: Data in January 1, 1986 Constant Dollars without AFUDC, but Including Contingency and Owner's Costs

(d) Units in the low end of the cost range burn low sulfur coal or are single units built on sites occupled by older coal-fired units
} 


\section{TABLE ES-2}

\section{ENERGY ECONOMIC DATA BASE \\ PHASE VIII UPDATE}

SUMMARY OF THE PHASE VIII UPDATE (1986) BASE CONSTRUCTION COSTS

(Constant January 1, 1986 Do1lars)

Designator

MWt (Rated)

MWe

Total Base

Construction Costs ( $\left.\$ \times 10^{6}\right)$

$(\$ / \mathrm{kWe})$
2438

1401

2190

1670

1225

2131
Coal-Fired

Power Plant $\underline{\mathrm{PWR} \cdot \mathrm{ME}(\mathrm{a})} \underline{\mathrm{PWR} \cdot \mathrm{BE}(\mathrm{a})} \mathrm{LSPB}(\mathrm{b})(\mathrm{c}) \underline{\mathrm{HS} 5(\mathrm{a})}$

$$
3417(d) \quad 3417(d) \quad 3500(d)
$$

1377

1144

1144

1311

488
573

1174

\footnotetext{
(a) Costs are based on an nth-of-a-kind, commercial unit

(b) Costs are based on a second-of-a-kind, pre-commercial unit

(c) Costs do not include the cost of the initial inventory of sodium

(d) Core Thermal Power
} 
TABLE ES-3

ENERGY ECONOMIC DATA BASE

PHASE VIII UPDATE

COMPARISON OF PHASE I (1978) AND PHASE VIII (1986) UPDATES

BASE CONSTRUCTION COSTS

(Constant January 1, 1986 Dollars)

\section{Designator}

EEDB Program Update Phase

MWt (Rated)

MWe

$\stackrel{1}{\omega}$

\section{Total Base}

Construction Costs $\left(\$ \times 10^{6}\right) 1087$

$(\$ / \mathrm{kWe})$

$\frac{\mathrm{PWR} \cdot \mathrm{ME}}{\frac{\mathrm{I}(\mathrm{a})}{3411(\mathrm{e})} \quad \frac{\mathrm{VIII}(\mathrm{b})}{3417(\mathrm{e})}}$

1139

1144

2438

955

2131

\begin{tabular}{|c|c|}
\hline \multicolumn{2}{|c|}{$\mathrm{PWR} \cdot \mathrm{BE}$} \\
\hline I (a) & VIII $(b$ \\
\hline $3411(\mathrm{e})$ & $3417^{(e}$ \\
\hline 1139 & 1144 \\
\hline 1087 & 1401 \\
\hline 955 & 122 \\
\hline
\end{tabular}

$\begin{array}{llll}\frac{L S P B}{\operatorname{VIII}(c)} & & \frac{\mathrm{HS} 5}{\mathrm{I}(\mathrm{d})} & \text { VIII (b) } \\ 3500(\mathrm{e}) & & 1379 & 1377 \\ 1311 & & 486 & 488\end{array}$

2190

$457 \quad 573$

1670

$940 \quad 1174$

(a) The Phase I Update (1978) PWR data model was the antecedent for both the PWR $\cdot M E$ and PWR BE data models

(b) Ratings were adjusted in the Phase VIII Update to reflect various technical changes, including the change from mechanical to natural draft cooling towers

(c) The LSPB has no Phase I Update antecedent for comparison because it was a new data model in the Phase VII Update; it is based on a second-of-a-kind, pre-commercial unit, while the PWR ME, PWR BE and HS5 are based on nth-of-a-kind, commercial units; costs do not include the cost of the initial inventory of sodium

(d) The Phase I Update (1978) 795 MWe HS8 costs were adjusted to reflect the costs for a 486 MWe HS5

(e) Core Thermal Power 
TABLE ES-4

ENERGY ECONOMIC DATA BASE

PHASE VIII UPDATE

PHASE I (1978) TO PHASE VIII (1986) UPDATE

BASE CONSTRUCTION COST INCREASES FOR THE

PWR $\cdot$ ME, PWR $\cdot$ BE AND HS 5

(Constant January 1, 1986 Dollars)

$\begin{array}{lccc}\text { PWR·ME } & \text { EEDB Program Update } & \begin{array}{c}\text { Average } \\ \text { Compound } \\ \text { Annual }\end{array} \\ \text { Rating } & 1139 \mathrm{MWe} & 1144 \mathrm{MWe} & \underline{\text { Pncrease (\%) }} \\ \text { Direct Costs } & 765 & 1153 & \underline{19} \\ \text { Indirect Costs } & \underline{322} & \underline{1285} & 19 \\ \text { Base Construction Costs } & 1087 & 2438 & 11\end{array}$

$\underline{\mathrm{PWR} \cdot \mathrm{BE}}$

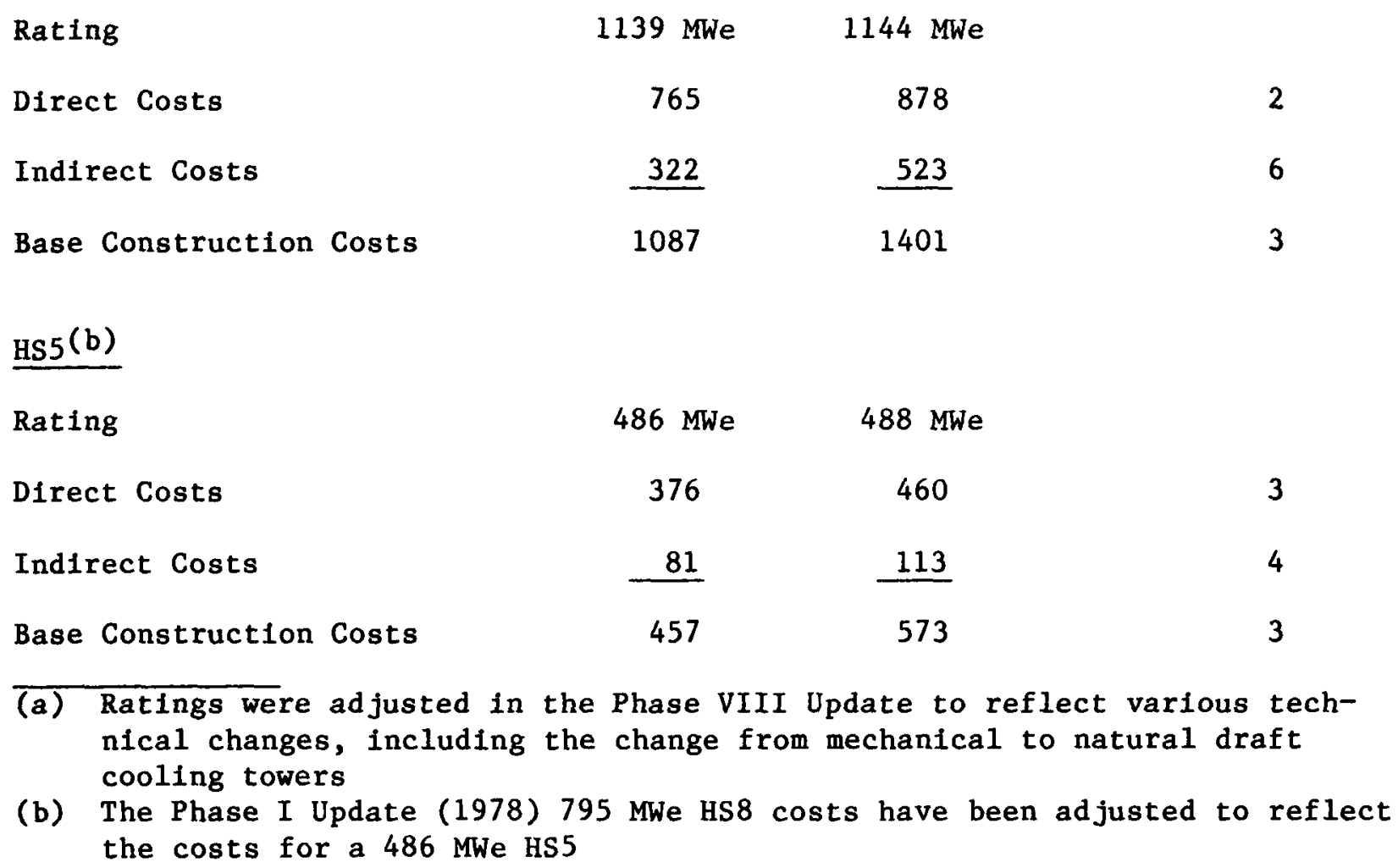


TABLE ES-5

ENERGY ECONOMIC DATA BASE

PHASE VIII UPDATE

COMPARISON OF PHASE I (1978) AND PHASE VIII (1986) UPDATES

PWR/HS5(a) BASE CONSTRUCTION COST RATIOS

PWR/HS5 Base

Construction

Cost (b) Ratio

Phase I Update (1978)

PWR

1.02

Phase VIII Update (1986)

PWR $\cdot M E$

$\mathrm{PWR} \cdot \mathrm{BE}$

1.82

1.04

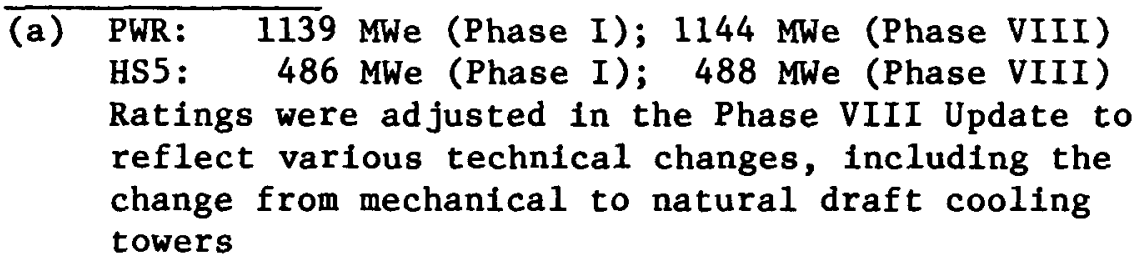

(b) Based on Constant Dollars per kWe in the Year of the Estimate; the Phase I Update (1978) 795 MWe HS 8 costs have been adjusted to reflect the costs for a 486 MWe HS5 
TABLE ES-6

ENERGY ECONOMIC DATA BASE

PHASE VIII UPDATE

LWR PLANT DESIGNS FOR THE FUTURE (LWR - F)

POTENTIAL SCHEDULE AND COST

\begin{tabular}{|c|c|c|c|}
\hline & $\underline{L W R} \cdot F(a)$ & $\mathrm{PWR} \cdot \mathrm{BE}$ (b) & $\mathrm{HS} 5(\mathrm{c})$ \\
\hline Rating (MWe) & 600 & 1144 & 488 \\
\hline $\begin{array}{l}\text { Licensing/Engineering/ } \\
\text { Construction Schedule (Months) }\end{array}$ & 70 & 120 & 88 \\
\hline $\begin{array}{l}\text { Base Construction Costs } \\
(1 / 1 / 86 \text { Constant Dollars })\end{array}$ & 733 & 1401 & 573 \\
\hline$\$ / k W e(1 / 1 / 86$ Constant Dollars) & 1222 & 1225 & 1174 \\
\hline
\end{tabular}

\footnotetext{
(a) Advanced Simplified Concept Light Water Reactor Nuclear Power Generating Station Based on Current Technology

(b) EEDB-Phase VIII Commercial Pressurized Water Reactor Nuclear Power Generating Station Based on Best Industry Experfence

(c) EEDB-Phase VIII Commercial High Sulfur Coal-Fired Fossil Power Generating Station
} 
SECTION 1

\subsection{OVERVIEW OF THE PHASE VIII UPDATE REPORT}

\subsection{EEDB PROGRAM BACKGROUND}

\subsubsection{Purpose and Objective}

The Energy Economic Data Base (EEDB) Program provides power plant technical and cost information to the U.S. Department of Energy (DOE) that is representative of current U.S. power plant construction cost experience. These data are used in program planning by the office of the Assistant Secretary for Nuclear Energy. The internally consistent, technical and cost data models for these large power plants ( 500 MWe to 1500 MWe) provide detailed design data and cost information as the basis for base construction cost estimates.

The objective of the data base is to develop credible cost estimates that clearly indicate what is included in the costs. This objective is achieved through the inclusion of a high level of detail in the technical data models, cleax statements of the ground rules and assumptions on which the technical and cost detail is based, and assembly and presentation of data in accordance with a uniform code-of-accounts. Additionally, the data models are periodically and extensively aligned with actual power plant technical practice and cost experience. Because of the level of detail, ground rules, code-ofaccounts and periodic alignments, the user may have confidence that the various alternatives contained in the data base are highly comparable, internally consistent, and representative of current expexience.

\subsubsection{Design and Application of the Data Base}

The data base is structured to provide point estimates of base construction costs (direct plus indirect costs) for each updated data model in a given update year. The estimates are based on quantities of commodities and equipment, unit costs, unit installation hours and labor rates that are representative of actual design and construction experience for each updated data model for the year of the update. The experience is gleaned from internal sources, field information, other cost reports and national cost indices. The most important and effective source of experience is the field information, which reflects the experiences of the entire power industry.

For any given update, the point estimates provide a valid and reasonably accuxate resource for making technical and cost comparisons of alternatives. The details in the technical data models provide a basis for determining cost related design feature differences. Analyses of these differences lead to an understanding and acceptance of the cost relationships among the compared alternatives. Such analyses may also show cost anomalies that identify opportunities for cost effective design improvements. Comparisons may be made among alternatives in the data base, between an actual plant and a data base model, or among two or more actual plants. When actual plants are 
compared, similar data base models are reconfigured to represent the actual plants before the comparison is made.

The point estimates generally represent the median of an actual or projected range of cost estimates for a particular data model. The succession of point estimates, over a period of several years, represents a trend rather than actual point to point variations. This is particularly true of the yearly variations in quantities of commodities, equipment and manhours. Year to year vartations reflect differences in the availability and accuracy of the experience resources, differences in the point in time at which the available data has been recorded and differences in the level-of-effort available for a particular update. The variations caused by these differences have exhibited the tendency to become normalized over the eight EEDB updates conducted over the last nine years.

\subsubsection{Historical Development}

The technical data models in the data base have evolved from studies performed by United Engineers \& Constructors Inc. over the last 22 years. The data base was assembled in 1978 by formally incorporating and updating the data from earlier studies. The resultant cost estimates and supporting technical descriptions were published in the EEDB Program - Phase I Update (1978) Report. 1 The EEDB has been updated and new update reports have been issued on an annual basis for the years 1979 through 1984.2-7 This report for the year 1986 describes the results of the elghth update in the EEDB Program.

Cost estimates based on updated technical data models recelve the primary emphasis in the update reports and may be used to generate the capital associated component of electric generating cost. In the earlier updates, this component was estimated and was added to fuel and operating/maintenance costs to predict present and future electricity costs. Beginning with the Phase VI (1983) Update, the effort was focused on estimating the base construction costs without predicting electricity costs. The Eighth (Phase VIII) Update has incorporated 1986 technical and economic data for a cost and regulation date of January 1, 1986. The Phase VIII Update of the EEDB Program was authortzed under ORNL Subcontract Number $43 \mathrm{X}-86004 \mathrm{~V}$.

\subsubsection{Recent Developments}

Each year, various technical and cost data models have been selected to be updated. Plant types and cost areas selected have been dependent on avatlable resources and related objectives of the Department of Energy. Data models for plant types and cost areas not included in an update were retained in the EEDB for possible reactivation in a future update. At the conclusion of the Seventh (Phase VII) Update, the EEDB contained technical and capital, fuel, and operating and maintenance cost data models for 33 different types and capacities of nuclear or fossil power generating stations; however, technical and cost data models for only four plant types were updated. 
The seven updates preceding this Eighth Update focused on a variety of power plant types and capacities at varying levels of effort. An historical record of the plant types and capacities included in each of these updates is given in Table 1-1. The existing data models selected for this update, the PWR $M E$, PWR $\cdot B E$, LSPB and HS5, are defined in Table 1-2.

\subsubsection{Resources for Use of the EEDB}

The EEDB Program Reference Book ${ }^{8}$ provides the assumptions, reference conditions, and ground rules under which the EEDB technical and cost data models have been developed. The results presented in the EEDB Update Reports are valid only under the described conditions. Therefore, a good understanding of the items included in and excluded from the data models is necessary before the results may be effectively extended to a wider range of conditions and sensitivities. A discussion of what is and is not included in EEDB Program reported costs may be found in the EEDB Program Reference Book and, in part, in Table 5-2 of this report.

The Program Reference Book provides important descriptive and tutorial information concerning the structure and use of the EEDB, particularly with respect to the data models' level of detall and the "code-of-accounts" system that provides order and consistency to that detail. It also contains reports of work done to support various aspects of earlier updates, together with significant reference data developed during those updates. The first three sections of the Reference Book describe the EEDB Program, identify the components of the data base and state the ground rules under which costs are developed and updated. Other sections describe the data base components and the technical and cost updating procedures in detail. The user of this Update Report is urged to review the Reference Book before attempting to utilize the data reported herein, in order to derive the maximum benefit from the Update. As a minimum, the user should be familiar with Sections 1 through 3 of the Reference Book.

\subsection{THE PHASE VIII EEDB PROGRAM OBJECTIVES, GOALS AND SCOPE}

\section{2 .1 objectives and Goals}

The objective of the Phase VIII Update of the EEDB was to support ongoing DOE assessment of the long-term economic potential of the nuclear option on a relative basis. The Phase IX Update will continue to pursue this objective. Consequently, the major goals for the Phase VIII Update were to maintain, expand and refine the technical/cost data models, to continue the development (started in the Phase VI Update) of cost's representative of the best nuclear power plant construction experience, to review advanced design concepts that may be used in future nuclear power plants and to lay the groundwork for Phase IX work. 


\section{2 .2 Scope}

The scope of the Phase VIII Update included three major efforts. The first major effort was the technical and cost updates of the PWR ME and HS5 data models. The PWR $\cdot \mathrm{ME}$ is the historic PWR data model with its cost history going back to the Phase I Update (1978). It reflects the median cost experience (ME) for nuclear power generating stations currently under construction. The HS 5 (488 MWe) is the lead comparison plant data model, having replaced the HS8 (791 MWe) in the Phase VII Update (1984). This change reflected the current industry trend for selection of lower coal-fired power plant capacities.

The second major effort for the Phase VIII Update was the technical and cost update and continued refinement of the PWR-BE data model. The PWR BE is based on the PWR $\cdot M E$, but has quantities of commodities, equipment, and installation manhours and indirect costs that reflect the best cost experience (BE) for nuclear power generating stations currently under construction. The PWR BE to HS5 cost comparison continues to provide an interesting and revealing contrast to the PWR $\cdot \mathrm{ME}$ to HS 5 cost comparison.

The third major effort for the Phase VIII Update was the extension and conformation (to EEDB ground rules) of technical detail for the LSPB. The EEDB LSPB is based on the concept developed by the Consolidated Management office for the LMFBR of the Electric Power Research Institute (EPRI/CoMO). It was first included in the EEDB and modified to generally meet EEDB ground rules during the Phase VII Update.

\subsection{SUMMARY OF PHASE VIII UPDATE ACTIVITIES}

Specifically, the activities pursued to meet the objectives and goals of the Phase VIII Update are listed below:

1. The EEDB was updated to include January 1, 1986 regulatory, technical and base construction cost data.

2. The PWR 'ME, PWR $\cdot B E$ and HS5 technical data models were updated, refined and expanded.

3. The LSPB was extended from the three-digit (summary) to the nine-digit (detailed) code-of-accounts level of detall.

4. An extensive and detailed technical review was made of the Structures and Improvements (Account 21) for the LSPB relative to the $\mathrm{PWR} \cdot \mathrm{ME} / \mathrm{BE}$, in connection with Item 3 above.

5. A detalled technical and economic review was made of the main condenser heat rejection system for the $\mathrm{PWR} \cdot \mathrm{ME} / \mathrm{BE}$, LSPB and HS5.

6. Coolling towers for all data models were changed from mechanical draft to natural draft type as a result of the review described in Item 5 . 
7. Building and equipment arrangement drawings and licensing/ engineering/construction milestone schedules were prepared for the LSPB and updated and refined for the PWR $M E / B E$ and HS5.

8. Heat balances were prepared for the LSPB and updated and refined for the PWR $M E / B E$, LSPB and HS 5 .

9. System design descriptions for the PWR ME/BE, LSPB and HS5 were updated, refined and expanded, based on Items 2 through 8 above.

10. The Phase VII Update (1984) Technical Reference Book ${ }^{9}$ was updated for the PWR $\cdot M E / B E$, LSPB and HS5, based on Items 1 through 9 above, and was issued as the Phase VIII Update (1986) Technical Reference Book.10

11. Indirect cost accounts were reviewed and refined for the $\mathrm{PWR} \cdot \mathrm{ME}, \mathrm{PWR} \cdot \mathrm{BE}, \mathrm{LSPB}$ and $\mathrm{HS} 5$ relative to craft, engineering and field supervision manhours.

12. Advanced LWR design concepts for future nuclear power plants were reviewed.

13. This update report was prepared based on Items 1 through 12 above.

The data tables, which make up a large part of this Phase VIII Update (1986) Report, were updated to the cost and regulation basis date of January 1 , 1986. The data in these tables supersede the corresponding technical and cost information presented in the Phase VII Update (1984) Report. 7

\title{
1.4 PRESENTATION OF THE EEDB PROGRAM REPORT
}

For the Eighth Update (1986), the EEDB Program Report is divided into two paxts.

\author{
This Volume: Energy Economic Data Base Program \\ Phase VIII Update (1986) Report \\ Companion Energy Economic Data Base Program \\ Volume: Technical Reference Book, Phase VIII \\ Update $(1986)^{10}$
}

These volumes are supplemented and amplified by the following five previously published EEDB report volumes:

Energy Economic Data Base Program

Phase VII Update (1984) Report ${ }^{7}$ 


\author{
Energy Economic Data Base Program \\ Phase VI Update (1983) Report \\ Energy Economic Data Base Program \\ Phase V Update (1982) Report ${ }^{5}$ \\ Energy Economic Data Base Program \\ Reference Book $(1982)^{8}$ \\ Energy Economic Data Base Program \\ Technical Reference Book $(1983)^{11}$
}

1.4.1 Purpose of the Phase VI (1983) and Phase VIII (1986) Technical Refexence Books

The purpose of the EEDB Technical Reference Book Phase VIII Update $(1986)^{10}$ is to provide the current technical design bases for each of the technical data models updated in the Eighth Update (1986). It contains a set of detailed stand-alone system design descriptions for each of these technical data models, which are supplemented with engineering drawings and licensing/ engineering/construction schedules. The system design descriptions reflect regulatory and industry practice and experience for nuclear and coal-fired power generating stations that were current on January 1, 1986.

The purpose of the EEDB Technical Reference Book (1983) 11 is to provide the technical destgn bases for the HS8 technical data model which was last updated in the Phase VI Update (1983). It contains detailed system design descriptions for the HS8, supplemented with engineering drawings. These system design descriptions reflect regulatory and industry practice and experience that were current on January 1, 1983.

The Table of Contents, the List of Tables and the List of Figures for the Phase VIII (1986) Technical Reference Book are included for reference in Table 1-3. Sections 1 and 2 discuss the technical data models and the assumptions and ground rules on which they are based. Sections 3 through 5 present the system design descriptions, engineering drawings and licensing/engineering/construction schedules. It is intended that the Technical Reference Book be periodically updated.

\title{
1.4.2 Purpose of the Program Reference Book and the Phase V (1982), Phase VI
} (1983) and Phase VII (1984) Update Reports

The purpose of the Program Reference Book, 8 which is a consolidatiaon of the Phase I (1978) through Phase IV (1981) Updates, and the Phase V (1982), Phase VI (1983) and Phase VII (1984) Update Reports $5,6,7$ is to provide the historical development of the EEDB through the Phase VII Update. These documents contain important descriptive and tutorial information concerning the structure and use of the EEDB. They also contain reports of work done to support various aspects of the first seven updates, $1-7$ together with significant reference data developed during those updates. 


\subsubsection{Purpose of the Phase VIII Update Report}

The purpose of the Phase VIII Update Report is to present the results of the current update, including a detailed description of technical and cost changes made to the data base following completion of the Phase VII Update. The report includes a detailed description of changes made to the technical data models, and summaries of the updated costs based on these changes. It is intended that a new Update Report be published for each update, using a standard format for easy cross referencing.

\subsection{ORGANIZATION OF THE PHASE VIII UPDATE REPORT}

This overview (Section 1) presents a general introduction to the EEDB Phase VIII Update. The remaining sections of the Update Report discuss and present the results of the Phase VIII Update at the level of detail necessary to convey the findings of the update activities.

Section 2 of this report presents a summary of the technical and cost update results.

Section 3 discusses the causes and effects of the Phase VIII Update cost changes, relative to the results of previous updates and to nuclear/coal-fired power plant cost trends. The section discusses costs and cost trends for nuclear power plants reflecting the median industry experience and the best industry experience.

Section 4 presents the details of the Phase VIII technical changes for each of the updated data models, including key technical parameters and quantities of commodities, equipment and labor manhours.

Section 5 presents the Phase VIII base construction costs for each of the updated data models. The direct cost changes, relative to the technical changes presented in Section 4, and the major indirect cost changes, relative to the available field information, are also discussed.

Section 6 presents the results of a review of advanced LWR concepts that may be utilized in future LWR nuclear power plants.

Section 7 contains the complete list of references cited in the Phase VIII Update Report. Definitions of terms and abbreviations used in this report are also included. 
TABLE $1-1$

ENERGY ECONOMIC DATA BASE

TECHNICAL DATA MODEL UPDATE HISTORY

NUCLEAR POWER GENERATING STATIONS

\begin{tabular}{|c|c|c|c|c|c|c|c|c|c|}
\hline \multirow[b]{2}{*}{ Plant Type } & \multirow[b]{2}{*}{$\begin{array}{c}\text { Net } \\
\text { Capacity } \\
\text { (MWe) } \\
\end{array}$} & \multicolumn{8}{|c|}{ EEDB Data Mode1(a) Number } \\
\hline & & $\begin{array}{l}\text { Initial } \\
\text { Update } \\
\text { (1978) } \\
\end{array}$ & $\begin{array}{c}\text { 2nd } \\
\text { Update } \\
\text { (1979) } \\
\end{array}$ & $\begin{array}{c}\text { 3rd } \\
\text { Update } \\
\text { (1980) } \\
\end{array}$ & $\begin{array}{c}\text { 4th } \\
\text { Update } \\
\text { (1981) } \\
\end{array}$ & $\begin{array}{c}\text { th } \\
\text { Update } \\
\text { (1982) } \\
\end{array}$ & $\begin{array}{c}\text { 6th } \\
\text { Update } \\
\text { (1983) } \\
\end{array}$ & $\begin{array}{c}\text { th } \\
\text { Update } \\
(1984) \\
\end{array}$ & $\begin{array}{c}\text { 8th } \\
\text { Update } \\
\text { (1986) } \\
\end{array}$ \\
\hline $\begin{array}{l}\text { Boiling Water Reactor } \\
\text { Plant - Median } \\
\text { Experience (BWR } \cdot M E)\end{array}$ & $\begin{array}{l}1190 \\
1195\end{array}$ & A1 & $\begin{array}{l}\text { A1 } \\
--\end{array}$ & $\begin{array}{l}\text { A1 } \\
--\end{array}$ & $\begin{array}{l}\text { A1 } \\
--\end{array}$ & Al & $\stackrel{\mathrm{A} 1 \cdot \mathrm{ME}}{--}$ & $\overline{-}$ & $\mathrm{Al} \cdot \mathrm{ME}(\overline{\mathrm{b}})(\mathrm{c})(\mathrm{d})$ \\
\hline $\begin{array}{l}\text { Boiling Water Reactor } \\
\text { Plant - Best Experience } \\
\text { (BWR } \cdot \mathrm{BE})\end{array}$ & 1190 & - & -- & -- & -- & -- & $\mathrm{A} 1 \cdot \mathrm{BE}$ & - & -- \\
\hline $\begin{array}{l}\text { High Temperature Gas } \\
\text { Cooled Reactor Plant } \\
\text { (HTGR) }\end{array}$ & 1330 & A2 & A2 & - & -- & - & -- & - & -- \\
\hline $\begin{array}{l}\text { High Temperature Gas } \\
\text { Cooled Reactor P1ant - } \\
\text { Steam Cycle (HTGR-SC) }\end{array}$ & 858 & - & -- & $\mathrm{A} 2$ (d) & A2 & $\mathrm{A} 2(\mathrm{e})$ & - & -- & -- \\
\hline $\begin{array}{l}\text { Pressurized Water Reactor } \\
\text { Plant - Median } \\
\text { Experience (PWR } \cdot \mathrm{ME})\end{array}$ & $\begin{array}{l}1139 \\
1144\end{array}$ & A3 & A3 & A3 & $\begin{array}{l}\text { A3 } \\
--\end{array}$ & $\begin{array}{l}\text { A3 } \\
--\end{array}$ & $\mathrm{A3}^{\mathrm{A} \cdot \mathrm{ME}}$ & $\stackrel{\mathrm{A} 3 \cdot \mathrm{ME}}{--}$ & $\mathrm{A} 3 \cdot \overline{\mathrm{ME}}(\mathrm{b})$ \\
\hline $\begin{array}{l}\text { Pressurized Water Reactor } \\
\text { Plant - Best Experience } \\
(\text { PWR } \cdot B E)\end{array}$ & $\begin{array}{l}1139 \\
1144\end{array}$ & -- & -- & - & $\begin{array}{l}-- \\
-\end{array}$ & $\overline{-}$ & $\mathrm{A} 3 \cdot \mathrm{BE}$ & $\mathrm{A} 3 \cdot \mathrm{BE}$ & $\overline{\mathrm{A} 3} \cdot \overline{\mathrm{BE}}(\mathrm{b})$ \\
\hline $\begin{array}{l}\text { Pressurized Heavy Water } \\
\text { Reactor Plant (PHWR) }\end{array}$ & 1162 & A4 & A4 & - & -- & - & - & -- & -- \\
\hline
\end{tabular}


TABLE $1-1$

ENERGY ECONOMIC DATA BASE

TECHNICAL DATA MODEL UPDATE HISTORY

NUCLEAR POWER GENERATING STATIONS

\begin{tabular}{|c|c|c|c|c|c|c|c|c|c|}
\hline \multirow{2}{*}{ Plant Type } & \multirow[b]{2}{*}{$\begin{array}{c}\text { Net } \\
\text { Capacity } \\
\text { (MWe) } \\
\end{array}$} & \multicolumn{8}{|c|}{ EEDB Data Mode1(a) Number } \\
\hline & & $\begin{array}{l}\text { Intitial } \\
\text { Update } \\
\text { (1978) } \\
\end{array}$ & $\begin{array}{c}\text { 2nd } \\
\text { Update } \\
\text { (1979) } \\
\end{array}$ & $\begin{array}{c}\text { rdd } \\
\text { Update } \\
(1980) \\
\end{array}$ & $\begin{array}{c}4 \text { th } \\
\text { Update } \\
\text { (1981) } \\
\end{array}$ & $\begin{array}{c}5 \text { th } \\
\text { Update } \\
\text { (1982) } \\
\end{array}$ & $\begin{array}{c}6 \text { th } \\
\text { Update } \\
\text { (1983) } \\
\end{array}$ & $\begin{array}{c}7 \text { th } \\
\text { Update } \\
\text { (1984) } \\
\end{array}$ & $\begin{array}{c}8 \text { th } \\
\text { Update } \\
\text { (1986) } \\
\end{array}$ \\
\hline $\begin{array}{l}\text { Pressurized Heavy Water } \\
\text { Reactor Plant (PHWR) }\end{array}$ & 1260 & - & -- & $\mathrm{A} 4$ (d) & A4 & $\mathrm{A} 4(\mathrm{e})$ & - & - & - \\
\hline $\begin{array}{l}\text { Gas Cooled Fast Reactor } \\
\text { Plant GCFR }(f)\end{array}$ & 917 & B1 & B1 & -- & -- & -- & -- & - & - \\
\hline $\begin{array}{l}\text { High Temperature Gas } \\
\text { Cooled Reactor Plant - } \\
\text { Process Steam (HTGR-PS) }\end{array}$ & 150 & - & -- & $B 1(d)$ & B1 & B1 & -- & - & - \\
\hline $\begin{array}{l}\text { Liquid Metal Fast Breeder } \\
\text { Reactor Plant - Median } \\
\text { Experience (LMFBR } \cdot M E \text { ) }\end{array}$ & $\begin{array}{l}1390 \\
1457\end{array}$ & $\begin{array}{l}\text { B2 } \\
--\end{array}$ & $A 5^{(d)}$ & $\overline{\text { A5 }}$ & $\overline{\mathrm{A} 5}$ & $-\overline{A 5}$ & $\mathrm{~A} 5 \cdot \overline{\mathrm{ME}}$ & -- & -- \\
\hline $\begin{array}{l}\text { Liquid Metal Fast Breeder } \\
\text { Reactor Plant - Best } \\
\text { Experience (LMFBR } \cdot B E \text { ) }\end{array}$ & 1457 & -- & - & - & - & -- & $\mathrm{A} 5 \cdot \mathrm{BE}$ & - & -- \\
\hline $\begin{array}{l}\text { Large Scale Prototype } \\
\text { Breeder Plant (LSPB) }\end{array}$ & $\begin{array}{l}1320 \\
1311\end{array}$ & -- & -- & -- & -- & -- & - & B3(d) & ${ }_{A 5}(\bar{b})(g)$ \\
\hline
\end{tabular}


TABLE $1-1$

ENERGY ECONOMIC DATA BASE

TECHNICAL DATA MODEL UPDATE HISTORY

COMPARISON POWER GENERATING STATIONS

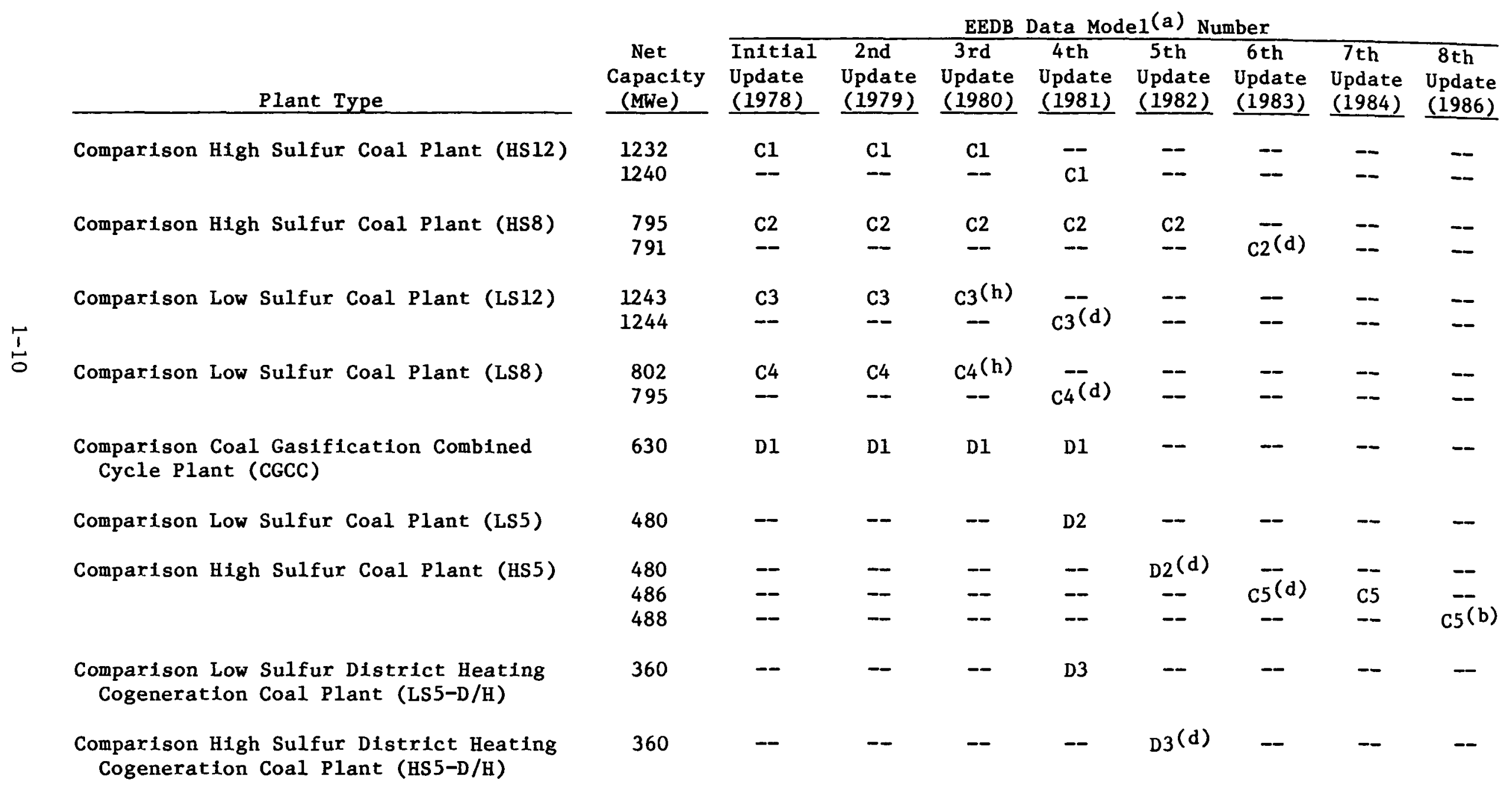


TABLE $1-1$

ENERGY ECONOMIC DATA BASE

TECHNICAL DATA MODEL UPDATE HISTORY

NOTES

(a) $\mathbf{A}=$ Detailed Data Mode1, Detailed Level Update

B = Summary Data Model, Summary Level Update

C = Detailed Data Model, Detailed Level Update

D = Summary Data Model, Summary Level Update

(b) Mechanical draft cooling towers were replaced with natural draft cooling towers causing a capactty change

(c) Phase VIII Update (1986) - BWR Supplement sponsored by U.S. Nuclear Regulatory Commission - Refer to Reference 12

(d) Indicates replacement of preceding data model

(e) Summary Level Update of Detalled Data Model

(f) GCFR data model was deleted from the data base in the Third Update (1980)

(g) Data model refined to fully conform to EEDB ground rules and site conditions

(h) Added flue gas cleaning to technical data model 
TABLE $1-2$

ENERGY ECONOMIC DATA BASE

NUCLEAR AND COMPARISON POWER GENERATING STATIONS FOR THE PHASE VIII UPDATE (1986)

EEDB Data Model (a)

Number $\quad$ Designator

A3 $\cdot$ ME

$\mathrm{A} 3 \cdot \mathrm{BE}$

A5

C5
PWR $\cdot$ ME

$\mathrm{PWR} \cdot \mathrm{BE}$

LSPB
Plant Type

Pressurized Water Reactor Plant

Pressurized Water Reactor Plant

Large Scale Prototype Breeder Plant

Comparison High Sulfur Coal Plant
Net

Capacity

1144 MWe

1144 MWe

1311 MWe

488 MWe

\footnotetext{
(a) A and C = Detailed Data Model, Detalled Level Update

ME = Median Industry Construction Experience Basis

$\mathrm{BE}=$ Best Industry Construction Experience Basis
} 
TABLE $1-3$

EEDB PHASE VIII UPDATE (1986) TECHNICAL REFERENCE BOOK

- TABLE OF CONTENTS

- TABLE LIST

- FIGURE LIST 


\section{ENERGY ECONOMIC DATA BASE (EEDB) PROGRAM \\ TECHNICAL REFERENCE BOOK \\ PHASE VIII UPDATE (1986)}

Legal Notice

List of Principal Contributors

i

List of Tables

List of Figures

Title

$\underline{\text { Page }}$

Section

Introduction

1-1

1.1 Technical Data Models

1-1

1.2 Data Base Reports

1.3 Organization of the Technical Reference Book

$1-1$

$1-3$

2.0

Rules and Procedures

$2-1$

2.1 Assumptions and Ground Rules

2.2 Design and Regulatory Criteria

2.3 Code-of-Accounts System

$2-1$

$2-4$

$2-8$

3-1

3.0 System Design Description for the 1144 MWe Pressurized Water Reactor Nuclear Power Generating Station (EEDB Program Technical Data Mode1 A3 - PWR)

3.1 Introduction

$3-1$

3.2 Design Criteria and Key Parameters

3-1

3.3 System Design Descriptions

3-9

3.4 Engineering Drawings

3.5 Design/Construction Schedule

$3-105$

$3-111$

4.0 System Design Description for the 1311 MWe

4-1

Large Scale Prototype Breeder Nuclear Power

Generating Station (EEDB Program Technical Data Model A5 - LSPB)

4.1 Introduction

4.2 Design Criteria and Key Parameters

4-1

4.3 System-Design Descriptions

4-1

4-11

4.4 Engineering Drawings

$4-96$

4.5 Design/Construction Schedule 
TABLE OF CONTENTS (cont'd)

\section{ENERGY ECONOMIC DATA BASE (EEDB) PROGRAM \\ TECHNICAL REFERENCE BOOK \\ PHASE VIII UPDATE (1986)}

Section

5.0

6.0

References, Definitions and Glossary

6.1 References and Bibllogxaphy

6.2 Definitions

6.3 Glossary of Acronyms and Abbreviations

5.1 Introduction

5.2 Design Criteria and Key Parameters

5.3 System Design Descriptions

5.4 Engineering Drawings

5.5 Design/Construction Schedule

Page

5-1

5-1

$5-1$

$5-8$

$5-88$

$5-98$

6-1

6-1

$6-4$

6-6

\section{Appendices}

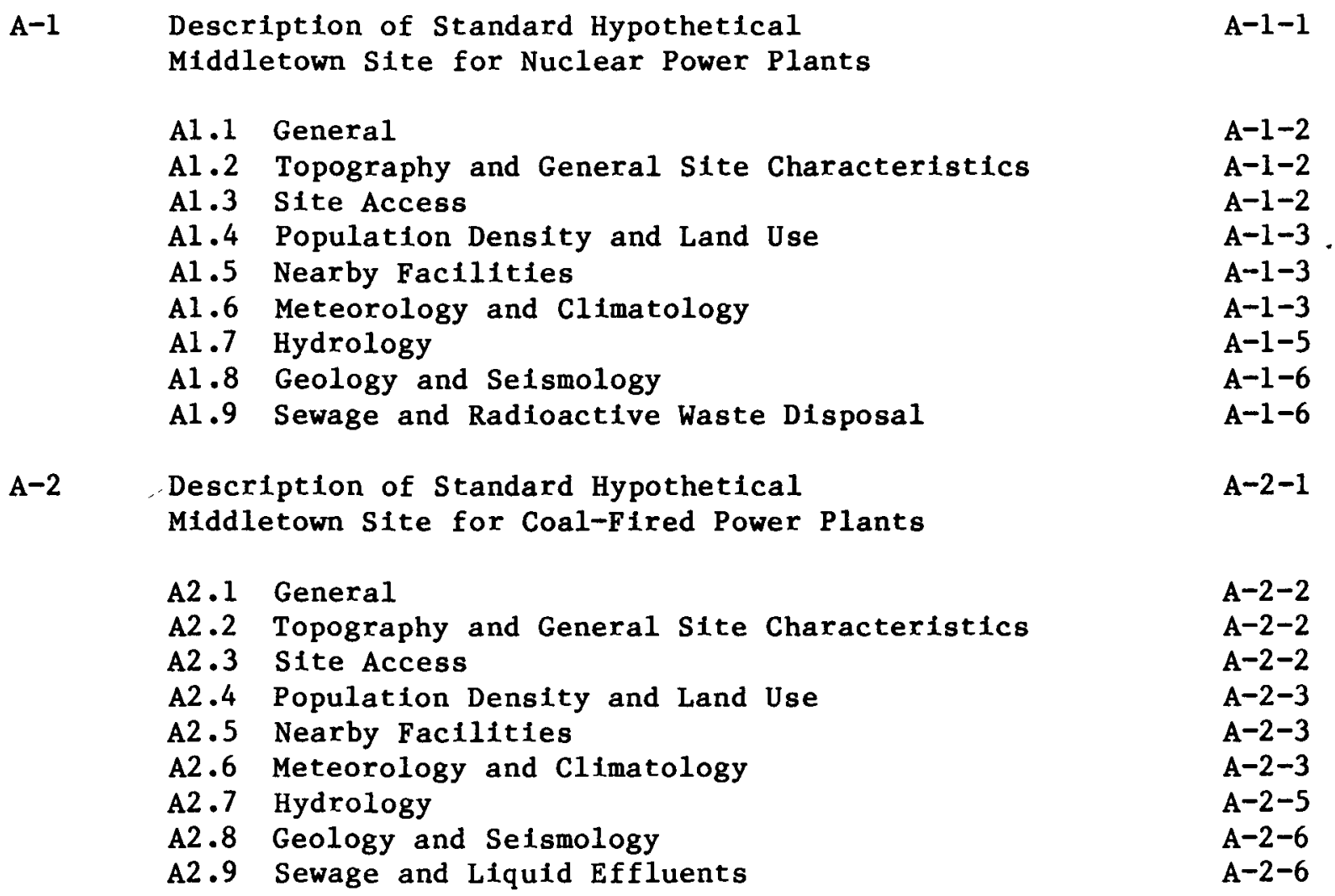




\title{
LIST OF TABLES
}

\author{
ENERGY ECONOMIC DATA BASE (EEDB) PROGRAM \\ TECHNICAL REFERENCE BOOK \\ PHASE VIII UPDATE (1986)
}

Table

Number

Title

$\underline{\text { Page }}$

1-1

Technical and Capttal Cost Data Models

$1-4$

Base Data Studies and Reports

1-2

Technical Data Model Update History

$1-6$

$1-3$

Nuclear and Comparison Power Generating Stations

$1-9$

for the Phase VIII Update (1986)

3-1

Pressurized Water Reactor

3-2

Nuclear Power Generating Station (PWR)

Technical Data Model Base Parameter Summary

$3-2$

1144 MWe Pressurized Water Reactor

$3-82$

Nuclear Power Generating Station (PWR)

Design Ambient Conditions for Electric Equipment

4-1 Large Scale Prototype Breeder

$4-3$

Nucleax Power Generating Station (LSPB)

Technical Data Model Base Parameter Summary

4-2

1311 MWe Large Scale Prototype Breeder

$4-72$

Nuclear Power Generating Station (LSPB)

Design Ambient Conditions for Electric Equipment

5-1 488 MWe High Sulfur Coal

$5-2$

Fossil Powex Generating Station (HS5)

Comparison Plant Technical Data Model

Base Parameter Summary

5-2 488 MWe High Sulfur Coal

Fossil Power Generating Station (HS5)

Design Ambient Conditions for Electric Equipment

5-3 488 MWe High Sulfur Coal

$5-97$

Fossil Power Generating Station (HS5)

Flue Gas Desulfurization System Flows

A1-1

Accident Meteorology for a Ground Level Release

$A-1-7$

Al-2

Accident Meteorology for an Elevated Release

$A-1-8$ 


\section{ENERGY ECONOMIC DATA BASE (EEDB) PROGRAM \\ TECHNICAL REFERENCE BOOK \\ PHASE VIII UPDATE (1986)}

\begin{tabular}{|c|c|c|}
\hline $\begin{array}{l}\text { Figure } \\
\text { Number } \\
\end{array}$ & Title & Page \\
\hline 3.1 & $\begin{array}{l}\text { Plot Plan - } 1144 \text { MWe PWR NPGS - } \\
\text { Middletown Hypothetical Site }\end{array}$ & $3-106$ \\
\hline 3.2 & $\begin{array}{l}\text { General Arrangement - } \\
\text { P1an at E1. 19'-0" (Ground F1.) - } \\
1144 \text { MWe PWR NPGS - } \\
\text { Middletown Hypothetical Site }\end{array}$ & $3-107$ \\
\hline 3.3 & $\begin{array}{l}\text { General Arrangement - } \\
\text { Plan at E1. } 73^{\prime}-0^{\prime \prime}\left(\text { Oper. F1.) }^{-}\right. \\
1144 \text { MWe PWR NPGS - } \\
\text { Middletown Hypothet1cal Site }\end{array}$ & $3-108$ \\
\hline 3.4 & $\begin{array}{l}\text { General Arrangement - } \\
\text { Sections "A-A" and "B-B" Elevations - } \\
1144 \text { MWe PWR NPGS - } \\
\text { Middletown Hypothetical Site }\end{array}$ & $3-109$ \\
\hline 3.5 & $\begin{array}{l}\text { Steam Heat Balance Diagram - } \\
1144 \text { MWe PWR NPGS - } \\
\text { Middletown Hypothetical Site }\end{array}$ & $3-110$ \\
\hline 3.6 & $\begin{array}{l}\text { Single Unit } 1144 \text { MWe Pressurized Water Reactor } \\
\text { Nuclear Power Generating Station (PWR) - } \\
\text { Preconstruction Permit Licensing Process Flow Diagram }\end{array}$ & $3-116$ \\
\hline 3.7 & $\begin{array}{l}\text { Single Unit } 1144 \text { MWe Pressurized Water Reactor } \\
\text { Nuclear Power Generating Station (PWR) - } \\
\text { Licensing Milestone Schedule }\end{array}$ & $3-117$ \\
\hline 3.8 & $\begin{array}{l}\text { Single Unit } 1144 \text { MWe Pressurized Water Reactor } \\
\text { Nuclear Power Generating Station (PWR) - } \\
\text { Engineering \& Construction Milestone Schedule }\end{array}$ & $3-120$ \\
\hline 4.1 & $\begin{array}{l}\text { Plot Plan - } 1311 \text { MWe LSPB NPGS - } \\
\text { Middletown Hypothetical Site }\end{array}$ & $4-97$ \\
\hline 4.2 & $\begin{array}{l}\text { Genexal Axrangement - } \\
\text { Plan at E1. 19'-0" (Ground F1.) - } \\
1311 \text { MWe LSPB NPGS - } \\
\text { Middletown Hypothetical S1te }\end{array}$ & $4-98$ \\
\hline
\end{tabular}


LIST OF FIGURES (cont'd)

ENERGY ECONOMIC DATA BASE (EEDB) PROGRAM

TECHNICAL REFERENCE BOOK

PHASE VIII UPDATE (1986)

Figure

Number

Title

Page

$4 \cdot 3$

General Arrangement -

4-99

Plan at E1. 88'-9" (Oper. F1.) -

1311 MWe LSPB NPGS -

Middletown Hypothetical Site

4.4

General Arrangement -

$4-100$

Section "A-A" Elevation -

1311 MWe LSPB NPGS -

Middletown Hypothetical Site

4.5

Steam Heat Balance Diagram -

$4-101$

1311 MWe LSPB NPGS -

Middletown Hypothetical Site

4.6

Single Unit 1311 MWe

4-107

Large Scale Prototype Breeder

Nuclear Power Generating Station (LSPB)

Preconstruction Permit Licensing

Flow Diagram

4.7

Single Unit 1311 MWe

4-108

Large Scale Prototype Breeder

Nuclear Power Generating Station (LSPB)

Licensing Milestone Schedule

4.8

Single Unit 1311 MWe

$4-111$

Large Scale Prototype Breeder

Nuclear Power Generating Station (LSPB)

Engineering and Construction Milestone

Schedule

5.1

Plot Plan - 488 MWe HSC FPGS -

5-89

Middletown Hypothetical Site

5.2

General Arrangement -

Plan at E1. 19'-0' (Ground F1.) -

Boiler \& Turbine Area -

488 MWe HSC FPGS -

Middletown Hypothetical Site 


\title{
LIST OF FIGURES (cont'd)
}

\author{
ENERGY ECONOMIC DATA BASE (EEDB) PROGRAM \\ TECHNICAL REFERENCE BOOK \\ PHASE VIII UPDATE (1986)
}

\begin{tabular}{|c|c|c|}
\hline $\begin{array}{l}\text { Figure } \\
\text { Number } \\
\end{array}$ & Title & Page \\
\hline 5.3 & $\begin{array}{l}\text { General Arrangement - } \\
\text { Plan at E1. } 64^{\prime}-0^{\circ}(0 \text { per. F1.) - } \\
\text { Boiler and Turbine Area - } \\
488 \text { MWe HSC FPGS - } \\
\text { Middletown Hypothetical Site }\end{array}$ & $5-91$ \\
\hline 5.4 & $\begin{array}{l}\text { General Arrangement - } \\
\text { Section "A-A" Elevation - } \\
488 \text { MWe HSC FPGS - } \\
\text { Middletown Hypothetical Site }\end{array}$ & $5-92$ \\
\hline 5.5 & $\begin{array}{l}\text { Steam Heat Balance Diagram } \\
488 \text { MWe HSC FPGS - } \\
\text { Middletown Hypothetical Site }\end{array}$ & $5-93$ \\
\hline 5.6 & $\begin{array}{l}\text { Flow Diagram - Balanced Draft System - } \\
488 \text { MWe HSC FPGS - } \\
\text { Middletown Hypothetical Site }\end{array}$ & $5-94$ \\
\hline 5.7 & $\begin{array}{l}\text { Flow Diagram - Coal Handling System - } \\
488 \text { MWe HSC FPGS - } \\
\text { Middletown Hypothetical Site }\end{array}$ & $5-95$ \\
\hline 5.8 & $\begin{array}{l}\text { Flow Diagram - Flue Gas Desulfurization } \\
\text { System - } 488 \text { MWe HSC FPGS - } \\
\text { Middletown Hypothetical Site }\end{array}$ & $5-96$ \\
\hline 5.9 & $\begin{array}{l}\text { States with Power Plant Site Certification } \\
\text { Authorities and/or Those Requiring a State } \\
\text { EIS/EA }\end{array}$ & $5-102$ \\
\hline 5.10 & $\begin{array}{l}488 \text { MWe High Sulfur Coal-Fired } \\
\text { Power Generating Station (HS5) } \\
\text { Licensing Activity and Chronology Diagram } \\
\text { Site Certification Stage (Group } 1 \text { States) }\end{array}$ & $5-103$ \\
\hline 5.11 & $\begin{array}{l}488 \text { MWe High Sulfur Coal-Fired } \\
\text { Power Generating Station (HS5) } \\
\text { Licensing Activity and Chronology Diagram } \\
\text { Power Plant Permitting Stage } \\
\text { (Group } 1 \text { and Group } 2 \text { States) }\end{array}$ & $5-104$ \\
\hline
\end{tabular}




\section{LIST OF FIGURES (cont'd)}

\section{ENERGY ECONOMIC DATA BASE (EEDB) PROGRAM TECHNICAL REFERENCE BOOK \\ PHASE VIII UPDATE (1986)}

Figure

Number

Title

Page

5.12

Single Unit 488 MWe High Sulfur

5-105

Coal-Fired Power Generating Station (HS5)

Licensing Milestone Schedule

5.13

Single Unit 488 MWe High Sulfur

$5-108$

Coal-Fired Power Generating Station (HS5)

Engineering \& Construction Milestone Schedule

A1.1

Temperature Duration Curves; Middletown, U.S.A.

$A-1-9$

A2 .1

Temperature Duration Curves; Middletown, U.S.A.

$A-2-7$ 


\section{SECTION 2}

\subsection{SUMMARY OF THE PHASE VIII TECHNICAL/COST UPDATE RESULTS}

\subsection{TECHNICAL SUMMARY}

The status of the technical data model base parameters for the Phase VIII Update is summarized in Table 2-1 for the nuclear power plants and Table 2-2 for the comparison power plant. The summaries present listings of important or key parameters that establish the technical envelope for the $P W R \cdot M E / B E$, LSPB and HS5. The system design descriptions and engineering drawings found in the Phase VIII Update Technical Reference Book ${ }^{10}$ supplement and support the key parameters.

\section{$2.2 \cos T$ SUMMARY}

Costs have been prepared for the EEDB as "overnight" base construction costs, which are the sum of the direct and indirect costs. Direct costs comprise equipment and commodity costs, the costs of necessary site material and labor for installation of the equipment and commodities. Indirect costs include the costs of construction services, engineering, engineering support, construction management, field supervision, quality assurance, insurance and taxes, and other expenses such as payroll taxes, overhead, and fees. EEDB base construction costs exclude those cost elements described in the EEDB Program Reference Book ${ }^{8}$ and in Table 5-2. They specifically exclude owner's costs, contingencles, escalation and allowance for funds used during construction. Direct, indirect and base construction costs are summarized in Table 2-3 for the updated power plant data models.

Tables 2-4 and 2-5 also summarize the same data for the updated technical/cost data models, except that the costs have been normalized to the identical electrical or thermal capacities, respectively. The normalization process is discussed in Section 6 of the Program Reference Book. The net electrical capacity chosen for this process is that of the EEDB PWR, so that the costs of the other technical data models can be compared to this most frequently chosen industry cost base. The nominal thermal capacity chosen for the normalization process is the maximum licensable nuclear power plant thermal rating of $3800 \mathrm{MWt}$, so that costs may also be compared on the basis of maximum licensable capacity.

Costs tabulated in Tables 2-3, 2-4 and 2-5 for the PWR $\cdot M E$, PWR $B E$ and HS5 are representative of nth-of-a-kind commercial units. The costs for the LSPB, although complying with EEDB Program ground rules and assumptions, are representative of a second-of-a-kind, precommercial unit. Consequently, LSPB costs are expected to decrease relative to the PWR ME, PWR'BE and HS5 when this data model is evolved to an nth-of-a-kind, commercial unit basis.

Al1 costs in this update report are presented as January 1, 1986 constant dollars. 
ENERGY ECONOMIC DATA BASE

PHASE VIII UPDATE

NUCLEAR POWER GENERATING STATIONS TECHNICAL DATA MODELS BASE PARAMETER SUMMARY

PWR - ME /BE (1144 MWe)

LSPB (1311 MWe)

\section{GENERAL}

1. Site

2. Operation

3. Technical Data Model Reference Date

Middletown, USA

Base Load

January $1,1986 *$

30

Single

$3,417 *$

$3,431 *$

$10,192 *$

$33.48 *$

1144 *

10
Middletown, USA

Base Load

January 1, 1986*

40

Single

3,500

3,542

$9,109 *$

37.46 *

1311 *

10

10. Water Table (Feet above mean river level)

Designates a Reference 7

(a) Includes Reactor Coolant Pumping Power

(b) Based on Rated Thermal Power

(c) Generator Step-up Transformer 
Effective Date $1 / 1 / 86$

Sheet 2 of 14

TABLE $2-1$

ENERGY ECONOMIC DATA BASE

PHASE VIII UPDATE

NUCLEAR POWER GENERATING STATIONS

TECHNICAL DATA MODELS BASE PARAMETER SUMMARY

$\mathrm{PWR} \cdot \mathrm{ME} / \mathrm{BE}$ (1144 MWe)

GENERAL (cont'd)

11. 100 Year Flood (Feet above mean river level)

12. External Missiles

\section{LICENSING}

13. Codes \& Standards Reference Date

January $1,1986 *$

\section{CIVIL/STRUCTURAI}

14.1 Containment

a. Type

b. Overall Height (ft)

c. Inside Diametex (ft)

d. Outside Dimension - Length \& Width ( $f t)$ *

e. Volume $\left(10^{6} \mathrm{cu} \mathrm{ft}\right)$ *
140

N/A

Reinforced Concrete

with Steel Plate Liner

219

$2.8($ Free $) *$
LSPB (1311 MWe)

Tornadoes Only

January 1,1986 *

Designates a data or description line change from the Phase VII Update (1984) Base Parameter Summary - Refer to Reference 7

N/A Not Applicable 
ENERGY ECONOMIC DATA BASE

PHASE VIII UPDATE

NUCLEAR POWER GENERATING STATIONS

TECHNICAL DATA MODELS BASE PARAMETER SUMMARY

$\mathrm{PWR} \cdot \mathrm{ME} / \mathrm{BE} \quad(1144 \mathrm{MWe})$

\section{CIVIL/STRUCTURAL (cont'd)}

f. Design Pressure (psig)

1. Inerted Cells

2. Areas except inerted cells
52

N/A

N/A

N/A

N/A

$\mathbf{N} / \mathbf{A}$

N/A

Enclosed

High Tuned

4/3 (Fuel)*
LSPB (1311 MWe)

N/A

10

3

Steel*

$95(e)$

$241 \times 200 *$

4.6

Enclosed

High Tuned

1.3 (Fue1/Blanket)*

17. Fuel or Fuel/Blanket Storage (Number of Cores)

* Designates a data or description line change from the Phase VII Update (1984) Base Parameter Summary - Refer to Reference 7

N/A Not Applicable

(d) The combination of the Confinement Structure, other confinement buildings (portions of nuclear island structures surrounding the contalnment designed for this purpose) and the nuclear island HVAC System are designed to 1imit radioactive release; "other confinement buildings" are not included in these parameters

(e) This height is the distance above the containment structure "roof" 
Effective Date $1 / 1 / 86$

TABLE $2-1$

\section{ENERGY ECONOMIC DATA BASE}

PHASE VIII UPDATE

NUCLEAR POWER GENERATING STATIONS

TECHNICAL DATA MODELS BASE PARAMETER SUMMARY

\section{$\mathrm{PWR} \cdot \mathrm{ME} / \mathrm{BE}$ (1144 MWe)}

CIVIL/STRUCTURAL (cont'd)

18. Flooding Provisions

19. Seismic SSE/OBE (g)

20. Foundations (Type)

a. Seismic Category I

b. Non-Seismic Category I

21. Grade Elevation (Feet above mean river leve1)

\section{MECHANICAL}

22. Reactor
a. Type

\author{
Cylindrical Carbon \\ Steel Pressure Vessel \\ with Stainless Steel \\ cladding, Hemispherical \\ Bottom Head and \\ Removable Hemispherical \\ Upper Head
}

LSPB (1131 MWe)

No Special Provisions

$0.25 / 0.125$

Mat

Spread Footings and/or Mats

18 
ENERGY ECONOMIC DATA BASE

PHASE VIII UPDATE

NUCLEAR POWER GENERATING STATIONS

TECHNICAL DATA MODELS BASE PARAMETER SUMMARY

PWR PME / BE (1144 MWe)

LSPB (1131 MWe)

MECHANICAL (cont'd)

b. Vessel Inside Diameter/Height (in/in)

$173 / 516$

$569 / 739$

c. Number Fuel Assemblies

193

372 (Drivex),

175 (Axial Blanket),

90 (Radial Blanket),

432 (Radial Shield),

36 (Control Assy.)/

6.2 (PItch)

d. Initial Core

$\mathrm{UO}_{2}$

e. Enrichment (\%)

3

f. Refueling Method
- Horizontal Tube Fuel* Transfer System Between Fuel Pool and Reactor Vessel

- Rail Mounted Refueling Platform with Telescoping Grapple over Fuel Pool
$(\mathrm{Pu}-\mathrm{U}) \mathrm{O}_{2}$

24.4 (Equilibrium Core)

- Rotating Plugs and* In-Vessel Through-the-* Head Refueling System*

- Tilting "A" - Frame* and Inclined Tube Fuel* Transfer System Be-* tween EVST and Reactor* Vesse1*

* Designates a data or description line change from the Phase VII Update (1984) Base Parameter Summary - Refer to Reference 7 


\section{TABLE 2-1}

ENERGY ECONOMIC DATA BASE

PHASE VIII UPDATE

NUCLEAR POWER GENERATING STATIONS

TECHNICAL DATA MODELS BASE PARAMETER SUMMARY

$\mathrm{PWR} \cdot \mathrm{ME} / \mathrm{BE}$ (1144 MWe)

MECHANICAL (cont'd)

23. Primary Fluid Type/Wt $\left(10^{5} 1 \mathrm{bs}\right)$

$\stackrel{N}{I} \quad 24$. Moderator

25. Reactor Coolant Conditions at

Reactor Outlet
a. Temperature $\left({ }^{\circ} \mathrm{F}\right)$
b. Pressure (psia)

c. Flow $\left(10^{6} 1 \mathrm{~b} / \mathrm{h}\right)$

26. Reactor Coolant/Recirculation Loops

a. Number of Loops/Number of Pumps per Loop
618

2,250

$140.6 *$

Light Water $/ 5.70$

Light Water

Four/One
LSPB (1311 MWe)
Liquid Sodium

(Primary and

Intermediate)/

52 (Primary)

32 (Intermediate)

(at $500^{\circ} \mathrm{F}$ )*

N/A

950

Near Atmospheric

(6 in-w.g.)

141.2

Four (Primary)/One

Four (Intermediate)/One

* Designates a data or description line change from the Phase VII Update (1984) Base Parameter Summary - Refer to Reference 7

N/A Not Applicable 
ENERGY ECONOMIC DATA BASE

PHASE VIII UPDATE

NUCLEAR POWER GENERATING STATIONS

TECHNICAL DATA MODELS BASE PARAMETER SUMMARY

$\mathrm{PWR} \cdot \mathrm{ME} / \mathrm{BE}$ (1144 MWe)

MECHANICAL (cont'd)

b. Drive Type/hp

$N$
1
$\infty$

27a. Intermediate Heat Exchangers*

a Number per Primary Coolant Loop*

b. Type*

c. Classification (Section III, * ASME B\&PVC) *

d. Total Heat Transfer Surface* (Effective) (SF)*

e. Total Coolant Flow at Rated* Load (Int. Loops) $\left(10^{6} 1 \mathrm{~b} / \mathrm{h}\right)$ *

27b. Steam Generators

a. Number per Coolant Loop
$\mathrm{N} / \mathrm{A}^{*}$

N/A*

N/A*

\section{Electric Motor $/ 7000$ \\ (hot) \\ Electric Motor $/ 9000$ \\ (cold)}

N/A*

N/A*

1
LSPB (1311 MWe)

\author{
Variable Speed Electric \\ Motor (Primary and \\ Intermediate)/ \\ 14,000 (Primary) \\ 7,000 (Intermediate)
}

$1 *$

She11 and Tube Type Heat* Exchanger*

Tube Side - Class $1 *$

Shell Side - Class 2*

$249,200 *$

$136.4 *$

* Designates a data or description line change from the Phase VII Update (1984) Base Parameter Summary - Refer to Reference 7

N/A Not Applicable 
ENERGY ECONOMIC DATA BASE

PHASE VIII UPDATE

NUCLEAR POWER GENERATING STATIONS

TECHNICAL DATA MODELS BASE PARAMETER SUMMARY

$\mathrm{PWR} \cdot \mathrm{ME} / \mathrm{BE}$ (1144 MWe)

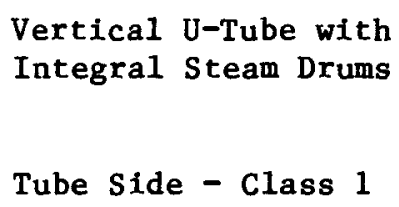

Vertical U-Tube with Integral Steam Drums

Tube Side - Class 1

c. Classification (Section III,

MECHANICAL (cont'd) ASME B\&PVC)

b. Type
LSPB (1311 MWe)

\author{
Benson Cycle Once- \\ Through Combined \\ Evaporator/Superheater \\ None (Steam generators \\ are external to the reac- \\ tor containment building \\ and separated from the \\ safety class portion of \\ the intermediate sodium \\ 10op; they are designed \\ to Section VIII of the \\ ASME B\&PVC) \\ $327,816 *$ \\ 12.6 \\ 2,290
}

Tandem-Compound,

6-Flow
Tandem-Compound, 6-Flow
$220,600 *$

$15.2 *$

1,000

Outlet Stean
Load (psia)

28. Turbine-Generator

a. Configuration 
Effective Date $1 / 1 / 86$

TABLE 2-1

ENERGY ECONOMIC DATA BASE

PHASE VIII UPDATE

NUCLEAR POWER GENERATING STATIONS

TECHNICAL DATA MODELS BASE PARAMETER SUMMARY

PWR - ME / BE (1144 MWe)

LSPB (1311 MWe)

MECHANICAL (cont'd)
b. Speed $(r / m i n)$
c. Last Stage Blade Length (in)

1,800

1,800

43

44

29. Main Steam Conditions at High Pressure Turbine Inlet (Rated Load)
a. Pressure (psia)
b. Temperature $\left({ }^{\circ} \mathrm{F}\right)$
c. Flow $\left(10^{6} 1 \mathrm{~b} / \mathrm{hr}\right)$ *

975

2,215

544

850

13.7

12.6

30. Gross Turbine-Generator Output at 2.5 In-HgA (MWe)*

1,192

$1,418 *$

31. Condensers
a. Type
b. She11/Divisions per She11
c. Arrangement
d. Number of Passes
e. Water Box

Single-Pressure

Single-Pressure*

$3 / 1$

Transverse

$3 / 1$

Two

Transverse

Sp1it

Two*

Split

* Designates a data or description line change from the Phase VII Update (1984) Base Parameter Summary - Refer to Reference 7 
TABLE 2-1

ENERGY ECONOMIC DATA BASE

PHASE VIII UPDATE

NUCLEAR POWER GENERATING STATIONS

TECHNICAL DATA MODELS BASE PARAMETER SUMMARY

LSPB (1311 MWe)

\section{MECHANICAL (cont'd)}
f. Pressure (In-HgA)
g. Total Heat Transfer Surface (SF)

2.5

$1,225,050 *$

$2.5 *$

$1,165,530$ *

32. Cooling Tower

$\stackrel{n}{1}$

\author{
Natural Draft* \\ Wet Evaporative \\ $2 / 717,000$ *
}

33. Cooling Tower Conditions
Natural Draft* Wet Evaporative
$2 / 711,400 *$

34. U1timate Heat Sink 
ENERGY ECONOMIC DATA BASE

PHASE VIII UPDATE

NUCLEAR POWER GENERATING STATIONS

TECHNICAL DATA MODELS BASE PARAMETER SUMMARY

PWR • ME / BE (1144 MWe)
2/Turbine

- 1/Emergency/Turbine

- 1/Emergency/Motor

None

$1 / 2$

$4 / 3,1 / 2$

One/Steam

$2 / 500,2 / 230$

$1,390 *$
LSPB (1311 MWe)

$2 /$ Turbine

- 2/Booster/Main Shaft*

- 1/Startup/Motor

One

$1 / 2$

$3 / 3$

One/Steam

$2 / 500,2 / 230$

38. Connection to off-Site Power (Number $/ \mathrm{kV}$ )

39. Generator

a. Rating (MVA) 


\section{TABLE 2-1}

ENERGY ECONOMIC DATA BASE

PHASE VIII UPDATE

NUCLEAR POWER GENERATING STATIONS

TECHNICAL DATA MODELS BASE PARAMETER SUMMARY

$\mathrm{PWR} \cdot \mathrm{ME} / \mathrm{BE}$ (1144 MWe)

ELECTRICAL (cont'd)
b. Voltage (kV)
c. Power Factor
d. $\mathrm{H}_{2}$ Pressure (psig)

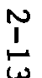

40. Generator Disconnect
a. Type
b. Rating (kV)

41. Auxilfary Power System
a. Mediun Voltage System A (kV)
b. Medium Voltage System B $(\mathrm{kV})$
c. Low Voltage System (V)
d. Direct Current Systems (V)

42. Unit Auxiliary Transformer

Nameplate Rating(f) (MVA)

\section{5}

0.9

75

Load Break Switch

36

$\begin{array}{ll}13.8 & 13.8 \\ 4.16 & 4.16 \\ 480 & 480 \\ 250 / 125 & 125 \text { and } 250 *\end{array}$

75
Load Break Switch

36

188

Designates a data or description line change from the Phase VII Update (1984) Base Parametex Summary - Refer to Reference 7

(f) Total of all transformers at top class of cooling rating 
Effective Date $1 / 1 / 86$

TABLE $2-1$

Sheet 13 of 14

ENERGY ECONOMIC DATA BASE

PHASE VIII UPDATE

NUCLEAR POWER GENERATING STATIONS

TECHNICAL DATA MODELS BASE PARAMETER SUMMARY

$\mathrm{PWR} \cdot \mathrm{ME} / \mathrm{BE}$ (1144 MWe) LSPB (1311 MWe)

ELECTRICAL (cont'd)

43. Reserve Auxiliary Transformer

44.1 Diese1-Generator Unit
a. Type/Number
Medium Speed/2
N/A
b. Voltage $(\mathrm{kV})$
4.16
N/A
c. $\operatorname{Rating}(\mathrm{kW} / \mathrm{PF})$
$6083 / 0.8$
N/A

44.2 Gas-Turbine Units

a. Standby Power Supply (Class 1E)
1. Number
N/A
2. Voltage $(\mathrm{kV})$
N/A
4.16
3. Rating (kW/PF)
N/A
$3000 / 0.9$

b. Emergency Power Supply (Non-Class 1E)

1. Number

N/A

Designates a data or description line change from the Phase VII Update (1984) Base Parameter Summary - Refer to Reference 7

N/A Not Applicable

(f) Total of all transformexs at top class of cooling rating 
Effective Date $1 / 1 / 86$

TABLE 2-1

\section{ENERGY ECONOMIC DATA BASE}

PHASE VIII UPDATE

NUCLEAR POWER GENERATING STATIONS

TECHNICAL DATA MODELS BASE PARAMETER SUMMARY

$\mathrm{PWR} \cdot \mathrm{ME} / \mathrm{BE}$ (1144 MWe)

ELECTRICAL (cont'd)

2. Voltage (V)

3. Rating (kW/PF)

45. Control Room Wiring

46. Multiplexing of Cables*

47. Instrumentation

Independent Sensors for Computer Input
LSPB (1311 MWe)

480

$1500 / 0.9$

Wired Directly to Panels in Control Room

Plant Wide/Distributed* Control System*

Independent Sensors for Computer Input

* Designates a data or description line change from the Phase VII Update (1984) Base Parameter Summary - Refer to Reference 7

N/A Not Applicable 
TABLE 2-2

\section{ENERGY ECONOMIC DATA BASE}

PHASE VIII UPDATE

COMPARISON POWER GENERATING STATION

TECHNICAL DATA MODEL BASE PARAMETER SUMMARY

HS5 (488 MWe)

\section{GENERAL}

1. Site

2. Operation

3. Technical Data Model Reference Date

4. Plant Life (years)

5. Number of Units

6. Rated Thermal Power (MWt)*

7. Net Plant Heat Rate (Btu/kWh)

8. Net Plant Efficiency (\%)

9. Net Power to GSU(a) (MWe)

10. Water Table (Feet above mean river level)

11. 100 Year Maximum Water Level (Feet above mean river level)

CIVIL/STRUCTURAL

12. Botler House

13. Turbine Butlding

14. Turbine Pedestal

15. Flooding Provisions

16. Seismic
Middletown, USA

Base Load

January 1, 1986*

30

Single

$1,377 *$

9,628 *

$35.44 *$

488*

10

8

Enclosed

Enclosed

High Tuned

No Spectal Provisions

Uniform Building

Code Zone 1

\footnotetext{
* Designates a data or description line change from the Phase VII Update (1984) Base Parameter Summary - Refer to Reference 7
}

(a) Generator Step-Up Transformer 
TABLE 2-2

\section{ENERGY ECONOMIC DATA BASE \\ PHASE VIII UPDATE}

COMPARISON POWER GENERATING STATION

TECHNICAL DATA MODEL BASE PARAMETER SUMMARY

HS5 (488 MWe)

CIVIL/STRUCTURAL (cont'd)

17. Foundations

18. Grade Elevation (Feet above mean rivex leve1)

\section{MECHANICAL}

19. Steam Generator Type

20. Steam Generator Outlet Conditions

a. Superheater Outlet at Maximum* Continuous Rating (MCR)* $\left(10^{6} \mathrm{lb} / \mathrm{h} / \mathrm{psig} /{ }^{\circ} \mathrm{F}\right)$

b. Superheater Outlet at Rated Load $\left(10^{6} \mathrm{lb} / \mathrm{h} / \mathrm{psig} /{ }^{\circ} \mathrm{F}\right)$

c. Reheater Outlet at Rated Load $\left(10^{6} \mathrm{lb} / \mathrm{h} / \mathrm{psig} /{ }^{\circ} \mathrm{F}\right)$

21. Forced Draft Fan
a. Number
2
b. Drive
c. Capacity (acfm boiler MCR)
Motor
489,000

Pulverized Coal Balanced

Draft Furnace

$3.97 / 2640 / 1010$

$3.5 / 2515 / 1010$

$3.2 / 530 / 1005 *$

22. Induced Draft Fan
a. Number
2
b. Drtve
Motor
c. Capacity (acfm boiler MCR)
867,000

* Designates a data or description line change from the Phase VII Update (1984) Base Parameter Summary - Refer to Reference 7 


\author{
ENERGY ECONOMIC DATA BASE \\ PHASE VIII UPDATE \\ COMPARISON POWER GENERATING STATION \\ TECHNICAL DATA MODEL BASE PARAMETER SUMMARY
}

HS5 (488 MWe)

MECHANICAL (cont'd)

23. Fue1 Type

a. Motsture (\% by wt)

b. U1timate Analysis (\% by dry wt)

c. Ash (\% by Dry Wt)

d. Calorific Value (Btu/1b)

24. Coal Delivery

25. Coal Storage

26. Coal Firing Rate at Rated Load (tons/day)

27. Coal Handling System

28. Number of Pulverizers/Spares

29. Stack
a. Height ( $f t)$
650
b. Gas Velocity (fps)
$40 \max$.

$\underline{\text { Eastern Coal }}$

11.3

Carbon $\quad 69.34$ Chlorine 0.04

Hydrogen 4.90 Sulfur 3.61

Nitrogen 0.86 Oxygen 9.65

11.6

As Received 11,026

Dry 12,432

100 Car Unit Train at 5 Hour Nominal Turnaround

60 Days at Rated Load,

8 Hours in Silos

5,112

Rotary Car Dumper, Coal

Breaker, Stacker/Reclaimer

Transfer Tower, Stacker/

Reclaimer, Crusher, Boiler

House Transfer Tower,

Trippers

$5 / 1$

* Designates a data or description line change from the Phase VII Update (1984) Base Parameter Summaxy - Refer to Reference 7 
TABLE 2-2

\section{ENERGY ECONOMIC DATA BASE}

PHASE VIII UPDATE

COMPARISON POWER GENERATING STATION

TECHNICAL DATA MODEL BASE PARAMETER SUMMARY

HS5 (488 MWe)

MECHANICAL (cont'd)

30. $\mathrm{SO}_{2}$ Scrubber
a. Type
b. Number of Modules/ Number of Spares

Lime (Wet) Preceded by*

$4 / 1$

31. Sludge Fixation

On-Site

32. Waste Disposal

Trucked off-site

33. Turbine-Generator
a. Configuration
Tandem-Compound, 4-Flow
b. Speed $(r / m i n)$
3600
c. Last Stage Blade Length (in)
30

34. Main Steam Conditions at High

Pressure Turbine Inlet (Rated Load)
a. Pressure, psia
2415
b. Temperature $\left({ }^{\circ} \mathrm{F}\right)$
1000
c. Throttle Flow $\left(10^{6} \mathrm{lb} / \mathrm{hr}\right)$
3.5

35. Gross Turbine-Generator Output at 2.5

in-HgA (MWe)

36. Condensers
a. Type
Single Pressure
b. Shell/Divisions per shell
$1 / 1$

\footnotetext{
* Designates a data or description line change from the Phase VII Update (1984) Base Parameter Summary - Refer to Reference 7
} 


\section{ENERGY ECONOMIC DATA BASE}

PHASE VIII UPDATE

COMPARISON POWER GENERATING STATION

TECHNICAL DATA MODEL BASE PARAMETER SUMMARY

HS5 (488 MWe)

MECHANICAL (cont'd)

c. Arrangement

Longitudinal

d. Number of Passes

1

e. Water Box

Split

f. Pressure (In-HgA)

2.5

g. Total Heat Transfer Surface (SF)

332,600

37. Cooling Tower
a. Type
Natural Draft Wet*
Evaporative
b. Number/Total Flow - Normal (gpm)
$1 / 239,000$

38. Cooling Tower Conditions
a. Approach $\left({ }^{\circ} \mathrm{F}\right)$
14
b. Range ( $\left.{ }^{\circ} \mathrm{F}\right)$
22
c. Wet $\operatorname{Bulb}\left({ }^{\circ} \mathrm{F}\right)$
74

39. Feedwater Pumps
a. Main (Number/Drive)
2/Turbine
b. Other (Number/Service/Drive)
2 /Booster/Motor

40. Feedwater Heaters
a Open Stages (Number)
One
b. High Pressure Closed Stages
(Number/Number Trains)
$2 / 1$

\footnotetext{
* Designates a data or description line change from the Phase VII Update (1984) Base Parameter Summary - Refer to Reference 7
} 
TABLE 2-2

\section{ENERGY ECONOMIC DATA BASE}

PHASE VIII UPDATE

COMPARISON POWER GENERATING STATION

TECHNICAL DATA MODEL BASE PARAMETER SUMMARY

MECHANICAL (cont'd)

$$
\begin{aligned}
& \text { c. Low Pressure Closed Stages } \\
& \text { (Number/Number Trains) }
\end{aligned}
$$

41. Stages of Reheat (Number/Type)

\section{ELECTRICAL}

42. Connection to Off-Site Power (Number/kV)

43. Generator
a. Rating (MVA)
$610 *$
b. Voltage $(\mathrm{kV})$
25
c. Power Factor
0.9
d. $\mathrm{H}_{2}$ Pressure (psig)
75

44. Generator Disconnect

45. Auxiliary Power System

a. Medium Voltage System A(kV)

b. Medium Voltage System B (kV)

c. Low Voltage System (V)

d. Direct Current Systems (V)

46. Unit Auxiliary Transformers

Nameplate Rating (b) (MVA)
None

4.16

480

$250 / 125$

$2 / 230,2 / 115$

Bolted Links

44

\footnotetext{
* Designates a data or description line change from the Phase VII Update (1984) Base Parameter Summary - Refer to Reference 7

(b) Total of all transformers at top class of cooling rating
} 


\title{
ENERGY ECONOMIC DATA BASE
}

PHASE VIII UPDATE

\author{
COMPARISON POWER GENERATING STATION \\ TECHNICAL DATA MODEL BASE PARAMETER SUMMARY
}

HS5 (488 MWe)

ELECTRICAL (cont $\left.{ }^{\prime} d\right)$

47. Reserve Auxiliary Transformer 22 Nameplate Rating(b) (MVA)

48. Diesel-Generator Unit
a. Type/Number
High Speed/2
b. Voltage (V)
480
c. Rating (kW/PF)
$400 / 0.8$
49. Control Room Wiring
Wired Directly to Panels in Control Room
50. Multiplexing of Cables
None
Independent Sensors for Computer Input

51. Instrumentation

\footnotetext{
* Designates a data or description line change from the Phase VII Update (1984) Base Parameter Summary - Refer to Reference 7

(b) Total of all transformers at top class of cooling rating
} 
TABLE 2-3

ENERGY ECONOMIC DATA BASE

PHASE VIII UPDATE

BASE CONSTRUCTION COST UPDATE SUMMARY

$\left(\$ 1986 \times 10^{6}\right)(a)$

Nuclear Plant Data Models

\section{Designator}

MWt (Rated)

MWe

Direct Cost

Indirect Cost

Base Construction Cost

$\$ / k W e$

\begin{tabular}{c}
\hline PWR $\cdot M$ \\
341 \\
1144 \\
1153 \\
1285 \\
2438
\end{tabular}

2131

$\mathrm{PWR} \cdot \mathrm{BE}(\mathrm{b})$

$3417(e)$

1144

878

$\underline{523}$

1401

2190

1225

1670

\section{Comparison Plant Data Model} HS5 (b)

1377

488

460

113

573

1174

(a) Data in January 1, 1986 Constant Dollars

(b) Costs are based on an nth-of-a-kind, commercial unit

(c) Costs are based on a second-of-a-kind, pre-commercial unit

(d) Reported costs do not include cost of the intitial inventory of sodium

(e) Core Thermal Power 
TABLE $2-4$

ENERGY ECONOMIC DATA BASE

PHASE VIII UPDATE

NORMALIZED (a) BASE CONSTRUCTION COST UPDATE SUMMARY $\left(\$ 1986 \times 10^{6}\right)(\mathrm{b})$

Nuclear Plant Data Models

\section{Designator}

MWt (Rated)

MWe

Direct Cost

Indirect Cost

Base Construction Cost

$\$ / \mathrm{kWe}$

$\$ / \mathrm{kWe}$

$\overline{\mathrm{PWR} \cdot \mathrm{ME} \$ / \mathrm{kWe}}$

\begin{tabular}{|c|c|c|}
\hline Nuc & Plant Dat & Models \\
\hline$\overline{\mathrm{PWR} \cdot \mathrm{ME}(\mathrm{d})}$ & $\mathrm{PWR} \cdot \mathrm{BE}(\mathrm{d})$ & LSPB $(e)(f)$ \\
\hline $3417(\mathrm{~g})$ & $3417(\mathrm{~g})$ & $3054(g)$ \\
\hline 1144 & 1144 & 1144 \\
\hline
\end{tabular}

1153

878

$\underline{523}$

1401

1225

0.57

1.00

2131

1399

$\underline{656}$

2055

1796

\footnotetext{
(a) Normalized to a plant size providing 1144 MWe (net)

(b) Data in January 1, 1986 Constant Dollars

(c) Normalization of HS5 is subject to greater error than for other models

(d) Costs are based on an nth-of-a-kind, commercial unit

(e) Costs are based on a second-of-a-kind, pre-commercial untt

(f) Reported costs do not include cost of the initial inventory of sodium

(g) Core Thermal Power
}

1014

886

0.42

Comparison Plant Data Model(c) HS5(d)

3228

1144

814

$\underline{200}$ 
TABLE 2-5

ENERGY ECONOMIC DATA BASE

PHASE VIII UPDATE

NORMALIZED(a) BASE CONSTRUCTION COST UPDATE SUMMARY

$\left(\$ 1986 \times 10^{6}\right)(b)$

Nuclear Plant Data Models

\section{Designator}

MWt (Rated)

MWe

Direct Cost

Indirect Cost

Base Construction Cost

$\$ / \mathrm{kWe}$

$\frac{\$ / \mathrm{kWe}}{\mathrm{PWR} \cdot \mathrm{ME} \$ / \mathrm{kWe}}$

1272

1272

0.57

1.00

2002

1150

$\begin{array}{lll}\frac{\mathrm{PWR} \cdot \mathrm{ME}(\mathrm{d})}{3800(\mathrm{~g})} & \frac{\mathrm{PWR} \cdot \mathrm{BE}(\mathrm{d})}{3800(\mathrm{~g})} & \frac{\mathrm{LSPB}(\mathrm{e})(\mathrm{I})}{3800(\mathrm{~g})}\end{array}$

1423

1550

727

2277

1600

0.80

Comparison Plant Data Model(c) HS5(d)

3800

1347

908

$\underline{223}$

1131

840

0.42

\footnotetext{
(a) Normalized to a plant size of 3800 MWt or its equivalent

(b) Data in January 1, 1986 Constant Dollars

(c) Normalization of HS5 is subject to greater error than for other models

(d) Costs are based on an nth-of-a-kind, commercial unit

(e) Costs axe based on a second-of-a-kind, pre-commercial unit

(f) Reported costs do not include cost of the initial inventory of sodium

(g) Core Thermal Power
} 
SECTION 3

\subsection{CAUSES AND EFFECTS OF THE PHASE VIII UPDATE CHANGES}

\subsection{INTRODUCTION}

The Phase VIII Update of the Energy Economic Data Base has developed base construction (direct plus indirect) costs for nuclear and coal-fired power plants, in terms of January 1, 1986 constant dollars. The EEDB Program provides this updated cost information on a periodic basis for use in other estimates of total generating costs. The updates are also used to indicate trends from which general conclusions may be made about future power costs.

High cost increases from update to update were identified for nuclear power plants during the first stx updates, particularly in the Phase $V(1982)$ and Phase VI (1983) Updates. Since the Phase VI Update and through the Phase VIII Update, however, these extreme cost increases appear to have moderated. This may be partly attributable to the fact that by January 1, 1986 all plants in the construction pipeline were nearing completion and several of the potentially most expensive units had been cancelled. In any event, because of theix timing and comprehensive nature, the Phase I (1978), Phase V (1982), Phase VI (1983) and Phase VIII (1986) Updates have become benchmarks through which significant cost drivers may be identified for the period from 1978 to 1986.

Recently constructed coal-fired power generating stations are smaller in size than those constructed during the early years of the EEDB Program. In recognition of this fact, the 791 MWe High Sulfur Coal-Fired Power Generating Station (HS8) was replaced with the 486 MWe (now 488 MWe) H1gh Sulfur Coal-Fired Power Generating Station (HS5) during the Phase VII Update as the lead comparison plant data model. This change is significant when comparing cost results on a dollar per kilowatt basis from the Phase VII and Phase VIII Update Reports with those of previous update reports.

\subsection{PERCEPTION OF NUCLEAR/COAL COST TRENDS}

In 1978, the year of the Phase I Update, the cost for electrictty was estimated to be significantly less from a nuclear power plant than from a coal-fired power plant of comparable capacity. However, when the Phase V Update (1982) was issued, the estimated costs of electricity from large nuclear and coalfired power plants were almost the same. Today, median to high cost experience light water reactor power plants are perceived by many to be not competitive with coal-fired power plants. Best to median cost experience plants axe perceived to be questionably competitive with coal-fired power plants. On the other hand, it appears that current best cost experience nuclear power plant designs could be competitive with coal-fired power plants when the necessary advanced design features are incorporated and the necessary government and industry actions are taken to encourage their deployment. 
A significant factor contributing to the decrease in economic advantage for nuclear power plants was their large and continuing cost increases between 1978 and 1986 compared with the smaller cost increases for coal-fired power plants. As was discussed in the Phase V, VI and VII Update Reports, $5,6,7$ the rapid rise in nuclear power plant costs was due primarily to large increases in the quantities of craft, engineering and fleld service manhours and to a lesser extent to increases in the quantities of commodities and equipment. These and other factors which are not evaluated in the EEDB analysis (such as mounting interest on funds used during construction, the ability to generate funds needed for construction, licensing delays, long construction times and the current moderate load growth) have essentially created a de facto moratorium on the construction of new nuclear power plants. No new nuclear power plants have been ordered since 1978 and none are forecast for the immediate future.

\subsection{RESULTS OF THE PHASE VIII UPDATE}

\subsubsection{EEDB and Actual Power Plant Costs}

The Phase VIII Update continued to focus on the principal nuclear power plant cost drivers when the overnight base construction costs of nuclear and comparison power plants were estimated for 1986. The base construction cost for the EEDB PWR $\cdot M E ~ 1144$ MWe data model, representing median light water reactor power plant cost experience, was estimated to be $\$ 2131 / \mathrm{kWe}$. The estimated base construction cost for the EEDB PWR BBE 1144 MWe data model, representing the best light water reactor power plant cost experience, was $\$ 1225 / \mathrm{kWe}$. The base construction cost for the EEDB HS5 488 MWe data model, representing average coal-fired power plant experience, was estimated to be $\$ 1174 / \mathrm{kWe}$. Details of the Phase VIII technical and cost data model updates are discussed in Sections 4 and 5 respectively.

Field information from confidential sources during the 1984/1985 time frame for nuclear and coal-fired power plants nearing completion indicate that the estimated nuclear power plant base construction costs in 1986 dollars without AFUDC, but including owner's costs and contingency, ranged from $\$ 1618 / \mathrm{kWe}$ to $\$ 3739 / \mathrm{kWe}$. The sources also indicate that base construction costs in 1986 dollars without AFUDC, but including owner's costs and contingency, for coal-fired power plants with flue gas desulfurtzation ranged from $\$ 648 / \mathrm{kWe}$ to $\$ 1942 / \mathrm{kWe}$. Plants at the low end of the cost range, however, were built on sites occupied by older coal-fired units or burned coal with a very low sulfur content. It should also be noted that the range of plant capacities and configurations varied more widely for the coal-fired power plants than for the nuclear power plants.

\subsubsection{Comparison of EEDB and Actual Power Plant Costs}

The relationship between industry reported costs in January, 1986 dollars and the EEDB estimated nuclear and coal-fired power plant base construction costs are as follows: 
EEDB Estimated Costs

EEDB Update

Phase VIII Update (1986)

Phase I Update (1978)

Phase VIII Update (1986) Adjusted(d)

Phase I Update (1978) Adjusted (d)

EEDB Update Period

Phase VIII Update (1986)

EEDB Adjusted Estimate Costs

Industry Reported Costs

\begin{tabular}{|c|c|c|c|c|}
\hline \multicolumn{3}{|c|}{ Constant $\$ / \mathrm{kWe}(1986)$} & \multicolumn{2}{|c|}{ Ratios } \\
\hline PWR -ME & $\mathrm{PWR} \cdot \mathrm{BE}$ & HS5 & $\begin{array}{c}\mathrm{PWR} \cdot \mathrm{ME} \\
\text { /HS5 }\end{array}$ & $\begin{array}{c}\mathrm{PWR} \cdot \mathrm{BE} \\
/ \mathrm{HS} 5\end{array}$ \\
\hline $\begin{array}{r}2131 \\
955\end{array}$ & $\begin{array}{c}1225 \text { (a) } \\
\text { (b) }\end{array}$ & $\begin{array}{l}1174 \\
940(c)\end{array}$ & $\begin{array}{l}1.82 \\
1.02\end{array}$ & $\begin{array}{l}1.04 \\
(\mathrm{~b})\end{array}$ \\
\hline $\begin{array}{l}2509 \\
1132\end{array}$ & $\begin{array}{c}1449(\mathrm{a}) \\
\text { (b) }\end{array}$ & $\begin{array}{l}1331 \\
1066(c)\end{array}$ & $\begin{array}{l}1.89 \\
1.06\end{array}$ & $\begin{array}{l}1.09 \\
\text { (b) }\end{array}$ \\
\hline
\end{tabular}

Industry Reported Costs $(d)$

\begin{tabular}{|c|c|c|c|c|}
\hline \multicolumn{3}{|c|}{ Constant $\$ / \mathrm{kWe}(1986)$} & \multicolumn{2}{|c|}{ Ratios } \\
\hline NPGS $\cdot M E$ & NPGS - BE & $\underline{F P G S} \cdot M E$ & $\begin{array}{l}\text { NPGS }{ }^{\circ} \mathrm{ME} / \\
\text { FPGS } \cdot \mathrm{ME}\end{array}$ & $\begin{array}{l}\mathrm{NPGS} \cdot \mathrm{BE} \\
\mathrm{FPGS} \cdot \mathrm{ME}\end{array}$ \\
\hline 2574 & $1618^{(a)}$ & $1340(\mathrm{e})$ & 1.92 & 1.20 \\
\hline 0.97 & 0.90 & 0.99 & -- & - \\
\hline
\end{tabular}

(a) Based on industry experience in 1983 when the PWR-BE was first created; the NPGS $\cdot B E$ value represents deterioration in that experience

(b) The Phase I Update PWR was representative of the PWR cost range in 1978 and is the predecessor to the Phase VIII PWR ME and PWR・BE, which are representative of the much wider PWR cost range in 1986

(c) The Phase I Update (1978) 795 MWe HS8 costs were adjusted to reflect the costs for a 486 MWe HS5

(d) Includes Owner's Costs and Contingency

(e) Based on units with scrubbers only

ME $=$ Median Cost Experience

$\mathrm{BE}=$ Best Cost Experience

NPGS = Nuclear Power Generating Station

FPGS = Coa1-Fired Power Generating Station

A comparison of the Phase VIII adjusted EEDB Estimated Costs with the Industry Reported Costs indicates reasonable agreement for both median and best cost experience cases, with the EEDB costs being on the order of 97 to 90 percent of the industry reported costs. This difference is within the uncertainty band for EEDB estimates when adjusted to reflect an owner's cost plus contingency. The 90 percent figure for the best experience comparison reflects the upward drift of best experience costs between 1983 when the PWR BE was first created and 1986. In order to meet the objective of providing a stable data model based on occurrence of regulatory reform and industry practice improvement, the EEDB PWR $B E$ has not been specifically adjusted to reflect this drift. The comparison shows that the primary EEDB objective that technical/cost data models remain reasonably representative of major nuclear power industry cost trends is met for the Phase VIII Update.

In earlier EEDB updates, the PWR data model was more representative of better cost experience than in later updates because the range of nuclear power plant 
costs was not as wide as in the later updates. Because of the cost range that evolved during recent years, the $P W{ }^{\cdot B E}$ was developed to reflect the best costs that the industry was experiencing, while the older PWR became the PWR $\cdot M E$ to reflect median industry cost experience. The early comparison plant data models represented best to median cost experience for coal-fired power plants. The HS8 in the Phase VI and earlier Updates and the HS5 in the Phase VII and later Updates continue to reflect best to median cost experience. The EEDB Estimated Cost Table above indicates PWR/HS 5 cost ratios. It is interesting to note that the $1986 \mathrm{PWR} \cdot \mathrm{ME} / \mathrm{HS} 5$ cost ratio has increased substantially since 1978, when nuclear power plants were considered to be competitive with coalfired power plants. On the other hand, the PWR BE/HS5 cost ratio is very close to the $1978 \mathrm{PWR} \cdot \mathrm{ME} / \mathrm{HS} 5$ cost ratio. The 1986 EEDB cost ratios are supported by the cost ratios shown in the Industry Reported Costs table above, within the uncertainty band of the adjusted EEDB estimates.

\subsubsection{EEDB and Actual Power P lant Cost Trends}

The Phase $V$ and Phase VI Updates 5,6 and associated field data identified the fact that nuclear power plant costs were rising at twice the rate of inflation and were being driven by rapidly increasing quantities of manhours and commodities. These increases were identified as being caused by a variety of conditions that are clear in hindsight, but could not have been anticipated by regulators and decision makers in the industry. Among them were rapid proliferation of regulations, codes and standards; owner/designer overreaction to the rapid appearance of these regulations, codes and standards; rework caused by field interferences, constantly changing designs in response to new requirements and inadequate engineering-to-construction lead times; extreme precision required in analyses, coupled with inflexible design and construction quality assurance requirements; management preoccupation with regulatory inspection, enforcement personnel site visits and lately prudency reviews; and low worker morale, caused by all of the above. Although these conditions led to increased commodities and base construction costs, their predominant impact was to increase craft labor, engineering and field service manhours.

During the Phase VII and Phase VIII Updates, it appeared that this trend was slowing. Consistent information, however, has become increasingly difficult to obtain. Although the rate of rise in manhours and costs has decreased, it is clear that the rise does continue during the Phase VIII Update. As previously mentioned, the rise now also seems to be affecting the best experience plants. Experience has shown that as time passes, the best experience plants have become more vulnerable to the conditions and delays experienced earlier by higher cost units. Conceptual designs for advanced LWR plants with simplified designs, more passive safety features, fewer components and reduced safety-class sprawl are currently being developed. It would appear that these plants have significant potential for removing the conditions that have created the escalation in manhours and the related cost increases. Section 6 provides a discussion of this subject. 


\subsection{BASE CONSTRUCTION COST TRENDS BETWEEN 1978 AND 1986}

\subsubsection{Base Construction Cost Components}

Base construction costs are controlled by the following five factors:

- quantities of commodities and equipment;

- unit installation manhours for commodities and equipment;

- home office, field supervision and construction service manhours shown as indirect costs;

- unit costs for commodities and equipment; and

- craft labor rates and professional and non-manual salary rates.

Total installation manhours are determined as the product of the first two items. Base construction costs are driven by variations in the first three items; the quantities of commodities, equipment and manhours. The last two items are mainly affected by inflation and amplify the cost levels determined by the first three items.

\subsubsection{Base Construction Cost Trends}

Figures $3.1,3.2$ and 3.3 show PWR and HS5 base construction cost trends from the Phase I Update (1978) through the Phase VIII Update (1986). The costs are normalized on the basis of dollars per kilowatt and are all in terms of 1986 constant dollars. The cost bars display costs for two important cost areas: "factory equipment plus site material" (commodities/equipment) costs and "labor" (craft, engineering and field supervision) costs. The accounting system used for these figures has a commodity/equipment/labor basis rather than the plant account basis reflected in Tables 2-3 through 2-5. For a detailed discussion of these accounts and what they contain, refer to Section 5.5 .

In 1978, the PWR point estimate represented the then current naxrow range of costs for PWR nuclear power plants. By 1983, that range had widened to the extent that a single point estimate was no longer representative of the range of costs. Consequently, the $\mathrm{PWR} \cdot \mathrm{ME}$ and $\mathrm{PWR} \cdot \mathrm{BE}$ estimates were developed to represent the median and best cost experience. Figures 3.1 through 3.3 show the cost trends that have taken place from the 1978 PWR to the $1984 / 1986$ $\mathrm{PWR} \cdot \mathrm{ME}$ and $\mathrm{PWR} \cdot \mathrm{BE}$.

Likewise in 1978, the HS8 (791 MWe) represented a medium capacity coal-fired power plant. In 1982 , the HS5 (486 MWe) was developed to reflect the trend to construction of smaller medium capacity coal-fired power plants. In 1984, the HS8 was no longer updated because the trend to smaller units persisted. Figures 3.1 through 3.3 show the cost trends that have taken place for an 
HS5 between 1978 and 1984/1986. This was accomplished by making adjustments to the 1978 HS8 costs to reflect an HS5 486/488 (the rating of the HS5 was changed to 488 MWe during the Phase VIII Update) MWe unit in that time frame. The adjustment added $\$ 188 / \mathrm{kWe}$ to the base construction costs of the 1978 HS in 1986 constant dollars.

Figure 3.1 shows the following:

- Commodities/equipment costs have grown by about 35,15 and 20 percent above inflation for the PWR $\cdot M E$, the PWR $\cdot B E$ and the HS5 respectively since 1978 .

- Total labor costs (craft, engineering and field supervision) have increased by about 220 percent above inflation for the PWR ${ }^{\circ} \mathrm{ME}$ between 1978 and 1986 .

- Total labor costs have increased by almost 40 percent above inflation for the PWR BE between 1978 and 1986 .

- Total labor costs have increased by more than 25 percent above inflation for the HS5 between 1978 and 1986.

By the Phase VI (1983) Update, it was apparent that labor costs had become the most predominant of the cost drivers that are investigated in the EEDB analyses. It is now also apparent that this trend has continued through the Phase VII and Phase VIII Updates, but at a slower pace. Since 1983, labor costs have caused nuclear power plants to become less and less competitive with coal-fired power plants. Since all labor rates in the figures have been adjusted for inflation, the labor cost increase is real.

This craft labor increase has been caused mostly by decreased productivity and to a lesser extent by rising quantities of commodities and equipment being installed. Craft labor productivity in nuclear power plants has two components. One is controlled by the workman and is related to his competence, thoroughness, organization and incentive to do quality work. The second is outside his control and is related to rework (design changes, interferences, inadequate lead times) and delays (extended schedules, quality assurance, inspections). It is the second component that predominates in the causes for decreased productivity.

It must be emphasized, however, that increases in indirect costs rather than in craft labor are the most significant part of the labor related increase for the $P W R \cdot M E$ data model. The indirect costs include the cost of labor associated with construction services, engineering/services and field nonmanual personnel. In the case of engineering/services, the increases are caused by proliferating regulations, codes and standards, repetitive changes in designs, escalating need for complex analyses and checking/rechecking of analyses, and increasingly rigorous quality assurance programs. In the case of the field non-manual personnel, the increases are caused by increasing interfaces with engineering, increasingly rigorous quality control programs, 
Increasing numbers of inspections, and the rising number of craftsmen to supervise.

\subsubsection{Equipment and Material Cost Trends}

Figure 3.2 shows the relationships and content of the commodity/equipment part of the cost bars in Figure 3.1. These equipment/material costs are discussed under the following three broad categories.

Items whose costs are primarily associated with manufacturers' prices: NF (nuclear or fossil steam supply system), $\mathrm{SR}$ (HS5 $\mathrm{SO}_{2}$ removal system), T-G (turbinegenerator unit), and $M$ (mechanical equipment, such as pumps, heat exchangers, tanks, insulation, and equipment for fire control, HVAC and water purification).

The overall costs in this group did not increase significantly above the inflation rate between 1984 and 1986 for the PWR data models or for the HS 5 data model. However, the lower than inflation tncrease for many ttems of mechantcal equipment offset a large increase in the cooling tower cost caused by the change from mechanical draft to natural draft type (refer to Section 4.3.2.2).

Between 1978 and 1984 , the steam supply system and the turbine-generator costs increased faster than the average inflation rate. During the same period, the $\mathrm{SO}_{2}$ removal system cost increased greatly because of design changes required to meet the 1977 (became effective in 1979) New Source Performance Standards. (This change applies equally to the HS5 and HS 8 data models even though the HS5 data model did not exist in the Phase I Update [1978].)

Items whose costs are primarily associated with quantity: $P$ (piping, including valves and supports), E (electrical equipment, wiring and raceways, instruments, and communication equipment), SS (structural support, which includes concrete with its formwork, retnforcing steel and embedded steel, and structural steel), and $S$ (other structural, including non-load bearing interfor and exterior wa1ls, elevators, painting, and excavation).

Between 1984 and 1986, these costs changed at essentially the inflation rate for all three data models. A more detailed analysis than may be gained from the figure showed increases that were somewhat less than the inflation rate. Some structural items previously shown separately from the mechantcal draft cooling towers, such as concrete and reinforcing steel for the cooling tower basins, are now included as mechanical equipment, because their costs are normally included in the scope of the natural draft cooling tower subcontract.

Between 1978 and 1984 , these costs increased faster than inflation by 27 percent for the PWR $\cdot M E$ and 20 percent for the HS5, while they nominally kept pace with inflation for the $\mathrm{PWR} \cdot \mathrm{BE}$. These increases reflect the rising quantities of commodities for the PWR $\cdot M E$, as discussed in the Phase V and VI Update Reports, 5,6 the FGD system changes for the HS5, and the relative constancy of commodity quantities for the PWR BE. 
Construction services ttems whose costs are indirect costs: CS (temporary buildings, temporary facilities, and construction tools and equipment).

This account grew at about the inflation rate between 1984 and 1986 for the PWR 'ME, PWR・BE and HS5 data models. Between 1978 and 1984 , however, this account increased at a rate exceeding inflation by about 130,30 and 10 percent for the $P W R \cdot M E, P W R \cdot B E$ and $H S 5$ respectively. These increases reflect lengthened construction schedules, an increase in temporary buildings for the increased field supervision personnel, an increase in tools and construction equipment to reflect the increase in craft labor, and the need for more temporary services (e.g., electric power distribution equipment).

\subsubsection{Labor Cost Trends}

Figure 3.3 shows the relationship and breakdown of the labor part of the cost bars in Figure 3.1. This figure emphasizes the extreme increase in labor costs above the inflation rate for the PWR PEE compared to the relatively small increases for the PWR $\cdot \mathrm{BE}$ and HS5 between 1978 and 1986.

\subsubsection{Labor Cost Trends From 1984 to 1986}

The overall increases in labor costs for the PWR ME, PWR BE and HS5 were relatively close to the general inflation rate between 1984 and 1986 . Generally, an increase in one area of site labor cost for the PWR $M E$ and $P W R \cdot B E$ was offset by small decreases in other areas. Following is a discussion of the 1984 to 1986 increases and decreases above or below general inflation in the various labor cost categories shown on Figure 3.3. Adjustments are included for the small changes in unit capacity caused by the 1986 change to natural draft cooling towers.

SC - Structural craft labor costs decreased by five percent for the PWR $M E$, less than one percent for the PWR $\cdot B E$ and $s i x$ percent for the HS5. The main reason for this decrease was the transfer of structural quantities for the cooling tower basin to the mechanical account for the change from mechanical draft to natural draft cooling towers, as discussed in Section 3.4.3. Some changes were also made in installation rates for various commodities based on $1984 / 1985$ field data. In the case of the PWR $B E$, these changes mostly offset the cooling tower basin related labor cost decrease.

MC - Mechanical craft labor costs increased by seven percent for the PWR ME, nine percent for the $\mathrm{PWR}^{\circ} \mathrm{BE}$ and three percent for the HS5. The principal reasons for these increases were the change from mechanical draft to natural draft cooling towers (as discussed in SC above) and an increase in the installation rate for small bore pipe in the PWR data models based on 1984/1985 field data.

EC - Electrical/I\&C craft labor rates increased by ten percent for the PWR $M E$, 12 percent for the $\mathrm{PWR} \cdot \mathrm{BE}$ and three percent for the HS5. These increases were generally caused by increases in the wire/cable quantities and installation rates based on $1984 / 1985$ field data. Additionally, the hourly pay for electricians increased at a rate greater than inflation. 
CS - Construction service craft labor costs did not change by more than two percent for any of the data models.

E - Engineering costs increased by 27 percent for the PWR ME caused by a 25 percent increase in engineering and home office service manhours and by hourly costs that increased faster than inflation. The increase in manhours was based on 1984/1985 field data. The PWR BE and HS5 engineering costs increased about one percent which was caused by hourly costs that increased faster than inflation.

FS - Field supervision costs increased by seven percent for the PWR $M E$, eight percent for the PWR・BE and three percent for the HS5. These increases were mainly caused by increases in site labor hours of four, seven and two percent respectively and by hourly costs for field supervision that increased faster than inflation.

0 - Other labor costs cover home and field office quality assurance and plant start-up and testing. These costs increased by 13 percent for the PWR $M E$, five percent for the $\mathrm{PWR} \cdot \mathrm{BE}$ and one percent for the HS5. The reasons for the increases are simflar to those given for the engineering and field supervision increases discussed in $E$ and FS above.

I\&T - Direct payroll taxes increased by two percent for the PWR ME, four percent for the PWR $B E$ and less than one percent for the HS5 because of the increase in craft labor hours identified in FS above.

\subsubsection{Labor Cost Trends from 1978 to 1984}

The increases in labor costs above general inflation for the three data models between 1978 and 1984 were 192 percent for the PWR・ME, 34 percent for the PWR $\cdot B E$ and 27 percent for the HS5. The following discussions provide reasons for the increases, particularly the very large increases for the $P W{ }^{\circ} M E$. These increases primarily reflect the rising quantities of manhours as well as the rising quantities of commodities for the PWR $M E$, as discussed in the Phase V and VI Update Reports.5,6 The comments refer to the PWR ME, unless noted otherwise.

SC - Structural craft labor costs increased by 73 percent between 1978 and 1984. This change resulted primarily from a significant decrease in installation labor productivity* and partly from an increase in quantities of structural commodities.

MC - Mechanical craft labor costs increased by 113 percent between 1978 and 1984, partly because of mechanical equipment and piping quantity increases, but primarily because of significant productivity decreases. Mechanical craft labor for the PWR is mainly utilized for the installation of the reactor,

* Productivity includes elements over which a worker has control (e.g., initiative, competence, interest) and elements over which the worker has no control (e.g., refer to Section 3.3.3.). 
piping, and other mechanical equipment. The HS5 mechanical craft labor increased by 33 percent primarily because of increases in the quantity and complexity of the equipment in the FGD system.

EC - Electrical/I\&C craft labor costs increased by 123 percent between 1978 and 1984, because of electrical/I\&C equipment and wiring quantity increases coupled with significant productivity decreases.

CS - Construction services labor costs to install the temporary buildings and facilities plus the other "craft labor" under indirect costs increased by 435 percent between 1978 and 1984 . These increases were partly caused by the conditions identified in the discussion of Item CS in Section 3.4 .3 and were partly caused by labor hour categories not previously identified. Similar causes were responstble for increases of 177 percent for the PWR BE and 28 percent for the HS5.

E - Engineering costs, which include payroll expenses, engineering support costs and other related costs, increased 145 percent from 1978 to 1984 . These increases resulted from the uncertainties caused by regulation and the inefficiencies of current practice (for a complete discussion of these effects refer to Appendix $C$ of the Phase VI Update Report ${ }^{6}$ ). Similar causes were responsible for increasing the PWR $\cdot B E$ by 32 percent. The HS 5 increase of 40 percent resulted primarily from the avallability of new and more complete data in 1983.

FS - Field supervision costs, which include payroll expenses and other related costs, increased by 846 percent from 1978 to 1984 . The dramatic increase in this account resulted from the greater number of craft laborers being supervised and, for the nuclear power plants, from the uncertainties caused by regulation and the inefficiencies of current practice (for a complete discussion of these effects refer to Appendix $C$ of the Phase VI Update Report ${ }^{6}$ ). Similar causes were responsible for increasing the PWR BE field supervision costs by 89 percent. The 41 percent increase in the HS5 field supervision costs was related primarily to increases in the number of craft laborers to supervise.

The field supervision (FS) plus engineering (E) costs now equal more than half of the total labor dollars required to build a new PWR $M E$. If the remaining indirect costs associated with labor (CS, 0 , and I\&T) are added to engineering and field supervision costs, the total 1986 labor related indirect costs equal 237 percent of the craft labor costs. These indirect-costs/craft-laborcost percentages were 217 percent in 1984 and 113 percent in 1978. For comparison, these indirect costs in 1986 equal 168 percent of the craft labor costs for the PWR BE and 69 percent of the craft labor costs for the HS5.

0 - Other labor costs cover home and field office quality assurance plus plant start-up and testing. Although this account grew significantly in terms of $\$ / \mathrm{kWe}$ from 1978 to 1984 , the account still represents less than five percent of the total labor cost for all three data models. 
I\&T - Insurance and taxes are a direct function of the cost for craft plus construction services labor. Between 1978 and 1984, however, the average percentage of wages to which insurance costs and taxes are applied increased to the extent that this account increased more rapidly than the direct labor account increased.

\subsubsection{Base Construction Cost Trend Recapttulation}

In summary, the Indirect labor costs have increased much more rapidly for the PWR $\cdot M E$ than for the PWR $\cdot B E$ and the HS5. Consequently, the total indirect costs are 111 percent of the direct costs for the PWR ME in 1986, versus 60 percent of the direct costs for the PWR $\mathrm{BE}$ and 25 percent of the direct costs for the HS5. The large rise in PWR ME labor costs may be attributed to continuation of the trends discussed in the previous section and verifled by industry experience. This experience includes low cost as well as high cost unit expertence. The low cost unit experience coincides with low craft, engineering and field supervision manhours as shown in Figures 3.2 and 3.3 .

Therefore, the PWR $B E$ and the HS5 data models showed similar increases in costs between 1978 and 1986, while the PWR ME costs increased at a significantly faster rate. This comparison implies that nuclear power is still a viable option (based on the method for calculating electricity costs given in the Phase V Update (1982) Report ${ }^{5}$ ), when the related regulatory reform and construction practice improvements discussed in the Phase V (1982) and Phase VI (1983) Updates 5,6 are implemented and/or when the advanced design features discussed in Section 6 are incorporated in future designs. 
FIGURE 3.1

ENERGY ECONOMIC DATA BASE (EEDB) PROGRAM

COMPARISON OF PHASE I (1978), PHASE VII (1984) AND PHASE VIII (1986)

PWR AND HS 5 BASE CONSTRUCTION COSTS

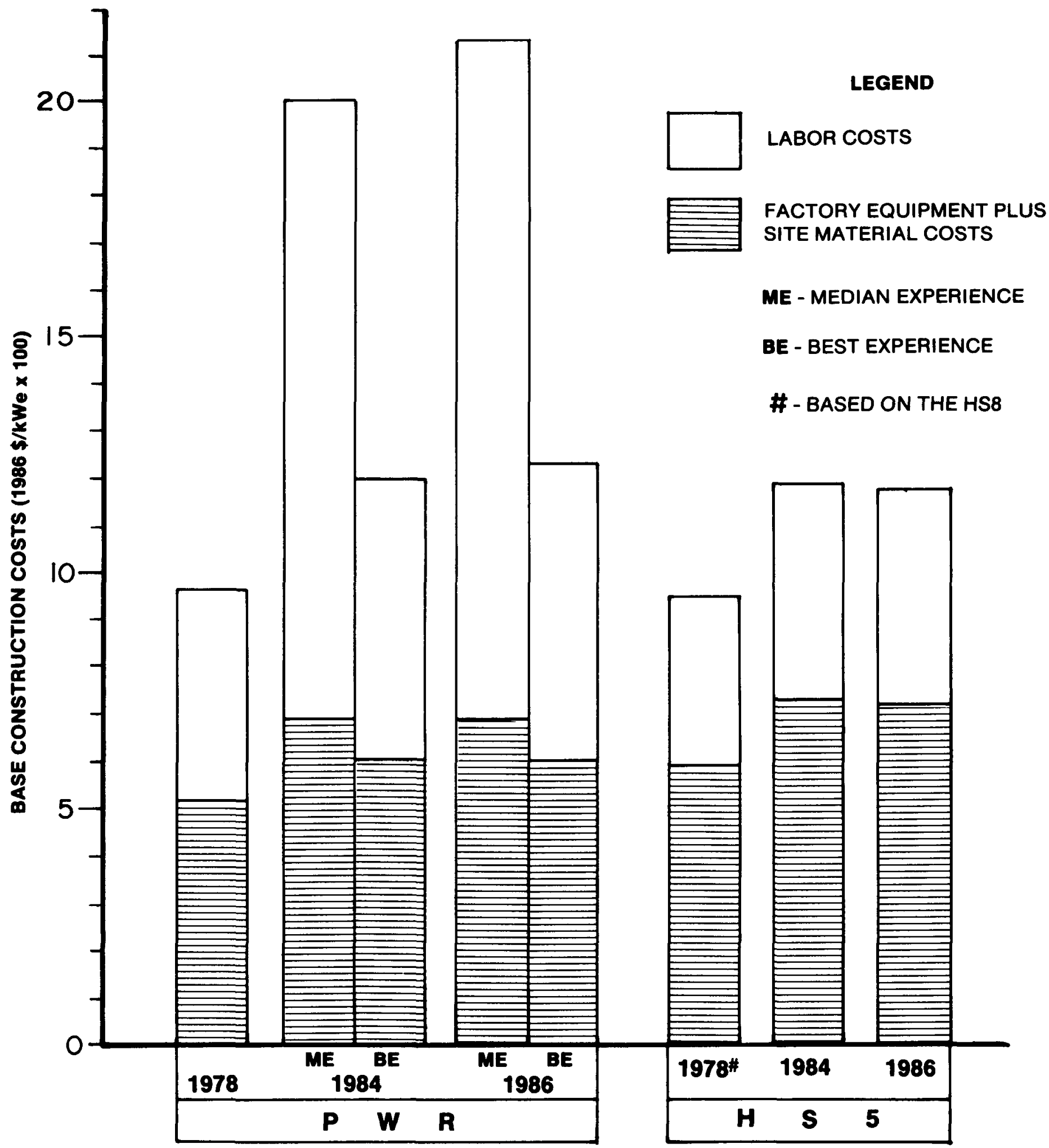

JANUARY 1 OF YEAR OF ESTIMATE 
FIGURE 3.2

ENERGY ECONOMIC DATA BASE (EEDB) PROGRAM

COMPARISON OF PHASE I (1978), PHASE VII (1984) AND PHASE VIII (1986)

PWR AND HS 5 FACTORY EQUIPMENT PLUS SITE MATERIAL COST

LEGEND (EQUIPMENT/MATERIAL)

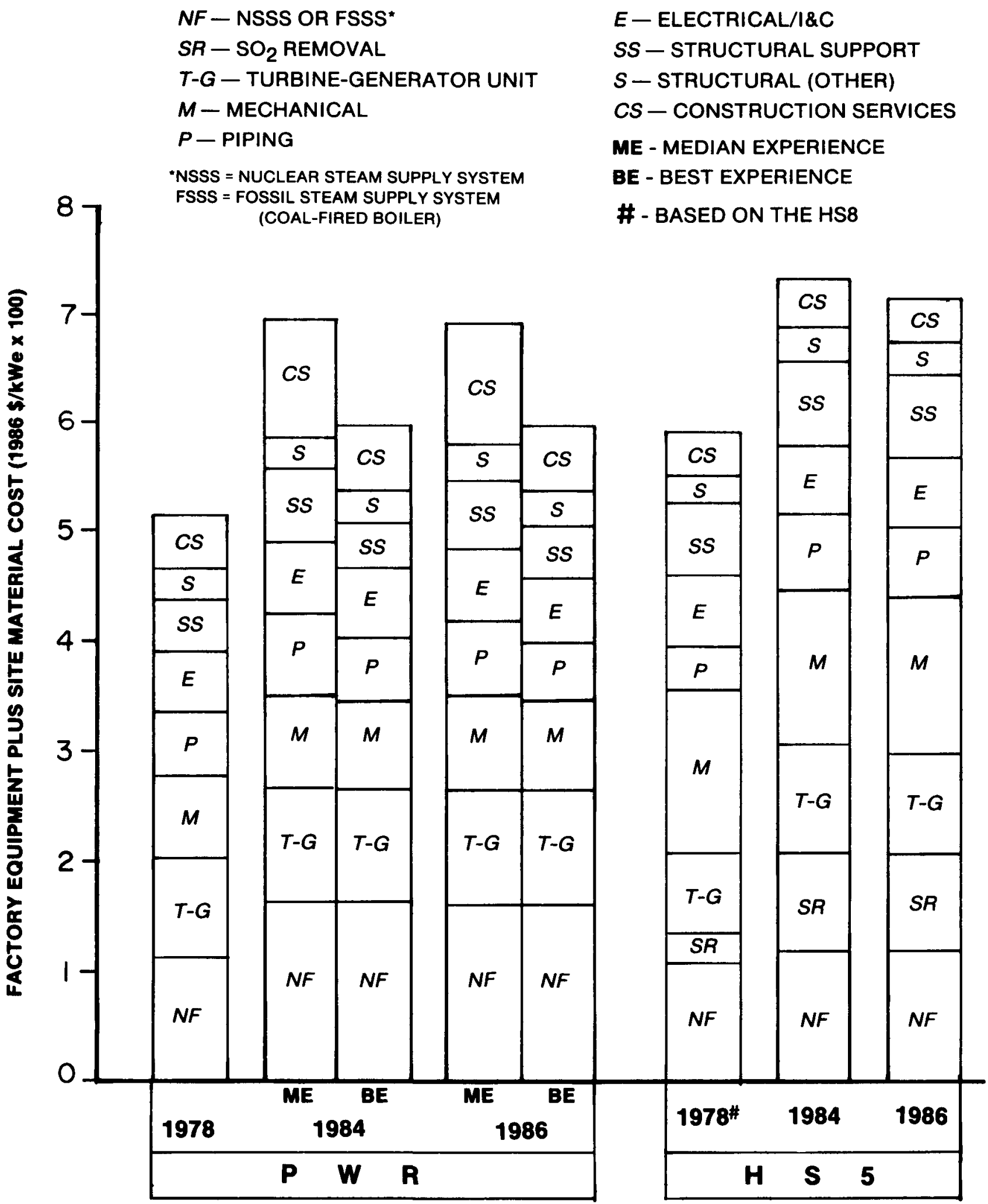

JANUARY 1 OF YEAR OF ESTIMATE 
FIGURE 3.3

ENERGY ECONOMIC DATA BASE (EEDB) PROGRAM

COMPARISON OF PHASE I (1978), PHASE VII (1984) AND PHASE VIII (1986)

PWR AND HS 5 LABOR COST

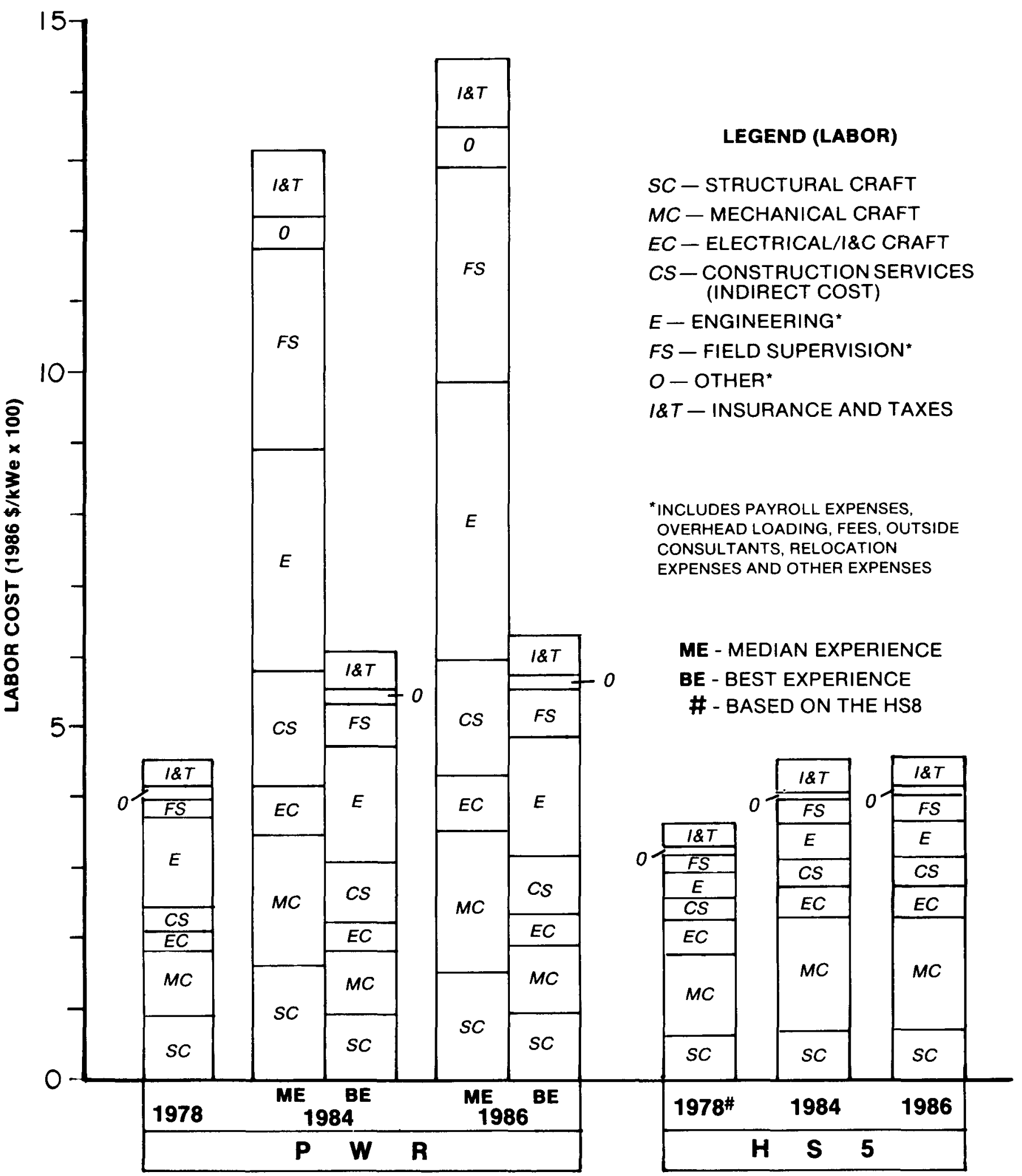

JANUARY 1 OF YEAR OF ESTIMATE 


\section{SECTION 4}

\subsection{TECHNICAL UPDATE FOR SINGLE UNIT NUCLEAR AND COMPARISON POWER PLANTS}

The Phase VIII Update of the Base Construction Costs only in the EEDB has been accomplished in two separate steps, in accordance with the EEDB Cost Update Procedure. The first step was the evaluation and adjustment or extension of the technical data models to assure that they reflected current power plant design practice and construction experience (including quantities of commodities, equipment and manhours), and current regulations, codes and standards. The second step was the adjustment of the cost data models to accommodate the technical data model revisions and to reflect the effects of equipment and labor cost escalation since the Phase VII (1984) Update. This section of the report presents the results of the detailed changes to or extension of the technical data models.

\subsection{TECHNICAL DATA MODEL UPDATE PROCEDURE}

A specific technical data model update procedure was developed for the EEDB, as a part of the cost Update Procedure. This procedure is described in Section 6 of the EEDB Program Reference Book. ${ }^{8}$ The procedure was utilized for the update of the PWR $\cdot \mathrm{ME}, \mathrm{PWR} \cdot \mathrm{BE}, \mathrm{LSPB}$ and HS5 technical data models listed in Table 1-2 of this report.

The Phase VIII Update performed a modified Level 2B (nine-digit code-of-accounts level of detail) Technical Update on these power plant technical data models. The PWR $\cdot M E, P W R \cdot B E$ and HS5 data models had been developed to the nine-digit code-of-accounts level of detail in previous updates. A major task of the Phase VIII Update was the extension of the LSPB data model from the three-digit (summary level) to the nine-digit (detalled level) code-of-accounts level of detail. The LSPB is a new data model which was included in the EEDB at the three-digit code-of-accounts level of detail during the Phase VII Update (1984). It was substituted for the LMFBR technical data mode1 previously included (Phase VI Update [1983]) in the data base.

Norma11y, a Level 2B technical update examines the major cost drivers: structures, nuclear or fossil steam supply system, turbine-generator unit, piping, electrical/I\&C, flue gas cleaning system (coal-fired power plants only) and condenser/cooling towers. In the Phase VIII update, many systems/equipment outside of that envelope were evaluated in order to assure current technical credibility for the PWR, LSPB and HS5. Sections 4.3.2, 4.4.1, 4.4.2 and 4.4.3 discuss the results of this evaluation.

During the Phase VIII Update, the BWR ME technical data model was updated under the sponsorship of the U.S. Nuclear Regulatory Commission. This activity included the update of the BWR $M E$ with a Mark III containment and the development of a BWR'ME with a Mark II containment. The BWR ME (Mark III) had been developed to the nine-digit code-of-accounts level of detail in previous updates and was last updated in the Phase VI Update (1983).6 The results of this work are given in the Phase VIII Update (1986) Report - BWR Supplement.12 
The BWR ME technical data model update was done in conjunction with and benefitted from the results of the Phase VIII PWR cost data model update. Consequently, the updated BWR $\cdot M E$ (Mark III) and BWR $M E$ (Mark II) technical data models and estimates are consistent with and comparable to the EEDB technical data models updated in the Phase VIII Update.

\subsection{TECHNICAL SUMMARY}

Summaries of the Phase VIII Update quantities of commodities and equipment are given for the PWR $\cdot M E$, PWR・BE, LSPB and HS5 in Table 4-1. Comparison summaries of Phase VII Update (1984) data are given in Table 4-2. The installed costs of the commodities/equipment summarized in these tables for the PWR $M E, P W R \cdot B E$, LSPB and HS5 represent approximately 75 percent of the total direct costs.

Summaries of the installed costs per unit of commodity or per item of equipment are given in Tables 4-3, 4-4, 4-5 and 4-6 for the PWR ${ }^{M}$, PWR $\cdot$ BE, LSPB and HS-5 respectively. These tables also show the distribution of dixect site labor between the various crafts. The data were provided in the same format in the Phase V (1982), Phase VI (1983) and Phase VII (1984) Update Reports $5,6,7$ for the $P W R \cdot M E$ and in the Phase VI and VII Update Reports for the PWR $\cdot B E$ and HS5. Information at this level of detail for the LSPB has not been previously available in the EEDB.

The Phase VIII Update changes in the quantities of commodities and equipment, as summarized in Tables 4-1 through 4-6, were a direct result of the technical changes described in the following sections.

\subsection{TECHNICAL UPDATE RESULTS}

The Phase VIII technical update supported the EEDB objective of assuring that the technical data models continue to represent current industry design and construction practice at the level established by the Phase VII Update. This objective was achieved by comparing commodity, equipment and manhour quantities for the updated EEDB technical data models with historical project experience, company confidential field data, and complementary and parallel studies.

\subsubsection{Review of Impact of Changing Regulations, Codes and Standards}

As a first step in the process of updating the technical data models to January 1, 1986, a review of new and revised regulations was conducted.13 As in the past two (1983 and 1984) updates, it was found for the Phase VIII Update that changes to existing regulations and promulgation of new regulations occurring since the previous update have had a negligible effect on the quantities of commodities and equipment in the technical data models.

For example, of the 153 Division 1 (Power Reactors) Regulatory Guides effective on January 1, 1986, six had been revised and two had been added during the period from 1984 to 1986. Additionally, two that were effective in 1984 had been withdrawn. Three of the revised regulatory guides were related to ASME 
Boller and Pressure Vessel Code Case Acceptability. Another three of the revised and the two new regulatory guides involved voluntary standards that reflected existing industry practice. Table 4-7 identifies the withdrawn, revised and added regulatory guides for the period. It also provides their projected impact on quantities and costs and the reason for the projections.

There was considerable activity from 1984 to 1986 in NRC Inspection and Enforcement Information Bulletins, Circulars and Notices, and Generic Letters with a total of 121 and 126 being issued during 1984 and 1985 respectively. Generally, these documents promulgated requirements that affected the administration of the engineering and construction of nuclear power plants, particularly in areas such as documentation, surveillance and testing. As such, they contributed to pressures that tended to increase craft, engineering and field supervision manhours, rather than changes to commodities and equipment.

\subsubsection{Review of Commodities and Manhours}

In the second step of the update process for the technical data models, overall detailed technical reviews were made against historical project and current field data. The reviews were conducted for the following purposes:

1. Improve and refine the data models in accordance with current practice and regulations.

2. Assure that the PWR and HS5 data models continue to reflect actual industry experience.

3. Expand the level of detail in the LSPB data model and improve its conformance to EEDB ground rules.

\subsubsection{PWR and HS5 Review}

An overall review was conducted on the PWR and HS5 to improve and refine the technical data models' design features and their associated quantities of commodities, equipment and installation manhours. The review was based on the Phase VII Update system design descriptions, building/equipment arrangement drawings and licensing/engineering/construction milestone schedules. It should be noted that the PWR $M E$ and the PWR $B E$ versions of the PWR are very similar with respect to site, building and system/equipment configuration. The principle differences in the two versions lie in their quantities of commodities, equipment and manhours.

The review resulted in a variety of refinements in the PWR and HS5 technical data models. These changes were reflected in the update of the system design descriptions, included in the Phase VIII Technical Reference Book, 10 and the commodity/equipment/manhour summaries included in Tables 4-1, 4-3, 4-4 and 4-6. The differences found in a comparison of the data in Tables 4-1 and 4-2 for the PWR $\cdot M E, P W R \cdot B E$ and HS5 are primarily a result of these refinements. The specific changes are listed in Sections 4.4 .1 and 4.4 .2 at the three-digit code-of-accounts level of detail. 
The increases in total craft manhours for the PWR $\cdot M E$, PWR $\cdot B E$ and HS 5 caused by these refinements are considered minor adjustments to achieve higher technical data model credibility. On a manhours per kilowatt basis, the PWR $\cdot$ ME manhours remain closely representative of craft manhours associated with the industry median cost experience. The PWR-BE manhours remain close to the best experience benchmark established during the Phase VI Update (1983). The HS5 manhours remain slightly higher than those associated with the industry median cost experience. The base construction costs for the HS5, however, remain closely representative of average coal-fired power plant expertence when compared on a dollar per kilowatt basis.

\subsubsection{General Review of Structures and the Main Condenser Heat Rejection System}

During the Phase VIII Update an extensive review was initiated of structural designs and quantities for major buildings. This effort had three purposes:

1. Review PWR and HS5 data to assure continued consistency between the technical designs (system design descriptions, arrangement drawings) and the representation of those designs (quantities of commodities and installation manhours).

2. Support the development of the LSPB structural design details required at the EEDB nine-digit code-of-accounts level of detail.

3. Assure the existence of an up-to-date EEDB structural design basis to support the BWR supplement effort mentioned in Section 4.1 .

The structural design review is expected to be extended to the remaining buildings for the PWR and HS5 and completed during the Phase IX Update (1987).

In particular, this review provided the basis for developing structural quantities (e.g. formwork, reinforcing steel, concrete, structural steel), architectural quantities (e.g. roofing, miscellaneous steel, exterior metal siding, interior walls) and their installation manhours from the CoMo design building dimensions and descriptions. This approach enabled the EEDB LSPB structures to reflect the CoMO LSPB design in accordance with EEDB practice and ground rules. Consequently, the CoMO structure design has been developed into a detailed accounting that is consistent and comparable with the other EEDB data models.

During the Phase VIII Update, a general review was also made of the Main Condenser Heat Rejection Systems. This review was focused on the use of mechanical draft cooling towers at the Middletown site. Recent plant design data indicates that cooling towers at nuclear power plants located on northeastern U.S. sites, such as Middletown, are of the natural draft type. The data also indicates that in all but the southwest regions of the U.S., natural 
draft cooling towers are utilized more often than mechanical draft cooling towers. Table 4-8 summarizes this data.

The EEDB has used mechanical draft cooling towers since the First Update (1978), because the studies 14 on which it was initially based used this type of tower. Generally, an increase of nearly 2-5 MWe in net capacity would be achieved for each technical data model if the mechanical draft cooling towers were replaced with natural draft cooling towers. The 2-5 MWe increase would be accrued from the elimination of the mechanical draft cooling tower fan auxiliary power requirement.

In the past, natural draft cooling tower factory equipment estimating quotations were significantly higher than those of mechanical draft cooling towers. The higher cost of natural draft cooling towers was sufficient to more than offset the capacity and energy charges for the cooling tower fans at the Middletown site. In recent years, however, the cost differences between the two types of towers have narrowed.

For the Phase VIII Update, the estimated installed cost, including auxiliaries, is lower for the natural draft cooling tower than for the mechanical draft cooling tower. Table 4-9 shows the advantages of the natural draft cooling tower over the mechanical draft cooling tower for the PWR on the Middletown site. Consequently, the decision was made to use natural draft cooling towers for all of the EEDB data models in the Phase VIII Update. As a result, the PWR capacity, which has held constant during the first seven updates, and the capacity of the HS5, which has held constant since its introduction in the Phase V Update (1982), have been changed as follows from their traditional values:

\section{Net Capacity (MWe)}

$\begin{array}{lll} & \text { PWR } & \text { HS5 } \\ \text { Prior to Phase VIII } & 1139 & 486 \\ \text { Phase VIII } & 1144 & 488\end{array}$

\subsubsection{LSPB Expansion}

An overall review and expanston of detail was conducted on the LSPB to place this technical data model on an equal basis with the other EEDB data models relative to extent of detail, degree of conformance with EEDB ground rules and level of consistency with other data models. The LSPB is based on the report, Large Scale Prototype Breeder, 15 a conceptual design developed by the Consolidated Management Office for the LMFBR of the Electric Power Research Institute (EPRI/CoMO) for the U.S. Department of Energy. During the Phase VII Update, the COMO LSPB was incorporated into the EEDB at the three-digit code-of-accounts level of detail and "moved" from the Idaho site, for which It was designed, to the EEDB Middletown site with a minimum of change. During the Phase VIII Update, it was extended to the nine-digit code-of-accounts level of detail and brought into full conformance with the EEDB ground rules. 
As a part of the extension and conformance effort, building/equipment arrangement drawings, a steam heat balance and licensing/engineering/construction milestone construction schedules were prepared. Additionally, the system design descriptions prepared during the Phase VII Update were revised. The arrangement drawings and heat balance were based on the Large Scale Prototype Breeder report, 15 EEDB ground rules and the Middletown site parameters. The milestone schedules were based on current field experience and EEDB ground rules.

These activities resulted in a variety of changes, as well as increased detail, for the EEDB LSPB. The changes were reflected in the update of the system design descriptions and other material included in the Phase VIII Technical Reference Book 10 and the commodity/equipment manhour summaries included in Tables 4-1 and 4-5. The differences found in a comparison of LSPB data in Tables $4-1$ and 4-2 are primarily a result of these changes and the increase in detail. The data in Table 4-2 for the LSPB was taken directly from the CoMO design documents. The data in Tables 4-1 and 4-5 for the EEDB LSPB was extracted from the detailed EEDB commodity/equipment/manhour lists developed at the nine-digit code-of-accounts level of detail.

A small decrease ( 0.6 percent) in net plant output from 1320 MWe for the CoMO LSPB to 1311 MWe for the EEDB LSPB also resulted from these changes. The difference is primarily caused by Idaho/Middletown site differences and by differences between the CoMO LSPB design basis and EEDB ground rules. In particular, the back-end of the heat cycle was revised to reflect the Middletown design wet bulb temperature of $68^{\circ} \mathrm{F}$ and the EEDB ground rule for a single pressure condenser. The Idaho site had a design wet bulb temperature of $63^{\circ} \mathrm{F}$ and the CoMO design utilized a multi-pressure condenser. Most of the capacity change was a result of the difference in the design wet bulb temperatures for the two sites. As a result of the changes, the EEDB LSPB net rating is on a consistent basis with the other EEDB technical data models.

A major increase (86 percent) in total craft manhours from $11.5 \times 10^{6}$ for the CoMO LSPB to $21.4 \times 10^{6}$ for the EEDB LSPB resulted from the extension/conformance changes. This was primarily due to differences in installation rates (installation manhours per untt of equipment/commodity) used by CoMO and EEDB and partly due to differences in the CoMO design basis and EEDB ground rules. A more recent CoMO LSPB estimate, however, has indicated that site labor has been increased to $19.7 \times 10^{6}$ craft manhours. 16

Examination of Tables $4-1$ and 4-2 reveals that the raceway quantity for the LSPB is significantly higher than that of the PWR BE, while the wire and cable quantity for the LSPB is about the same as for the PWR BE. Because the CoMO LSPB utilizes distributed microprocessors for control and data acquisition, the ratio of power cable to instrumentation and control cable is significantly higher for the LSPB than for the PWR. Power cable bulk and heat dissipation requirements are stronger drivers of raceway volume than control and instrumentation cable space requirements. Therefore, the LSPB feet of raceway per feet of cable is higher than for the PWR. Although hard wiring is the ground rule for the EEDB, distributed microprocessors were incorporated into the EEDB LSPB to reflect the CoMO design. 


\subsection{DETAILED TECHNICAL CHANGES}

The EEDB Technical Reference Book, 10 prepared during the Phase VIII Update, contains the base data for each of the technical data models updated. The base data axe in the form of assumptions, ground rules, design criteria, system design descriptions, engineering drawings and milestone schedules.

This section describes the modifications made to the Phase VII technical data models required for the Phase VIII Update and preparation of the Technical Reference Book. Commodities, equipment and craft labor manhours and costs resulting from this update are tabulated for each of the four mature (ninedigit code-of-accounts level of detail) techntcal data models in Tables 4-3 through 4-6 as follows:

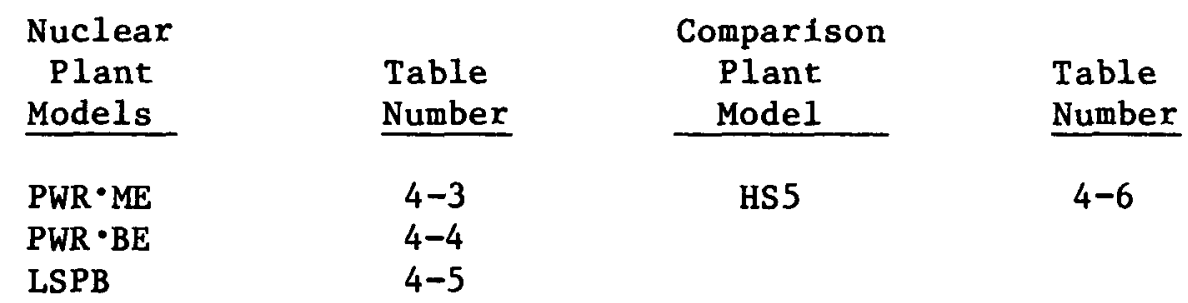

The following pages discuss the specific technical data model modifications made during the Phase VIII Update. The discussions of these modifications are given at the three-digit code-of-accounts level of detail. A parenthetical statement is provided at the end of each modification description to indicate the effect of the modification on the base construction costs. For convenience, the discussion of each technical data model is staxted at the top of a new page. 
4.4.1 EEDB Data Mode1 Number A3 -ME, Type PWR -ME, EEDB Phase VIII Update (1986) EEDB Data Mode1 Number $A 3^{\cdot} B E$, Type PWR ${ }^{\circ} B E$, EEDB Phase VIII Update (1986)

The following technical data model modifications are common to the two PWR nuclear power generating stations selected for the Phase VIII Update. These modifications take the form of changes in quantities of commodities and equipment and their installation manhours, which are an integral part of the technical data models. In many cases, however, the quantities differ for the $P W R \cdot M E$ and $P W R \cdot B E$ to the extent that median and best industry experience differ. This is particularly true for installation manhours. The changes are based on current designs and construction practice as reflected in the Phase VIII technical reviews, ongoing assessments of field data and United Engineers' experience.

\section{General}

During the Phase VIII Update review, a discrepancy in the feedwater heater No. 1 drain cooler outlet temperature was discovered. This was corrected, but the result was a reduction of approximately 0.4 MWe in generator output. Although this is a small decrease, it was considered important to maintain generator output consistent with that previously reported in order to maintain data model continuity. To do so, the turbine throttle flow was increased 0.15 percent and a new heat balance calculated.

This heat balance revision necessitated mtnor changes to various equipment in the reactor plant, turbine plant and main condenser heat rejection system. A description of each change and its effect on cost is included in the following overall discussion of changes at the three-digit level of detail.

ACCOUNT 212 Reactor Containment Building

A review of the containment liner plate was conducted which resulted in a definition of the quantity and an increase in the cost. A mini-spectification was added to the plant equipment list to clarify the composition of the listed lot of material. A review of the installation manhours indicated that no changes should be made. (Cost Increase)

The control rod drive cooling system supply fans were changed to exhaust fans. The type of fan was changed from belt driven centrifugal to vaneaxial, based on a review of the design. (No Cost Change)

ACCOUNT 218B Administration and Service Building

The quantity of auxiliary boiler room exhaust fans was changed from one to two based on a review of the design. (Cost Increase)

ACCOUNT 218H Non-Essential Swttchgear Building

Quantities of some structural commodities were decreased based on a general review of this structure. (Cost Decrease) 
ACCOUNT 220A Nuclear Steam Supply System

Reactor core thermal power was changed from 3411 MWt to 3417 MWt and NSSS thermal power was changed from $3423 \mathrm{MWt}$ to $3431 \mathrm{MWt}$, as a result of the heat balance corrections. (Negligible Cost Increase)

ACCOUNT 222 Main Heat Transfer and Transport System

The steam generator capacity (total of four) was changed from $15.1 \times 10^{6}$ $\mathrm{lb} / \mathrm{hr}$ to $15.2 \times 10^{6} \mathrm{lb} / \mathrm{hr}$ in accordance with the revised heat balance. (Cost Increase)

ACCOUNT 224 Radioactive Waste System

The solid waste system was revised to reflect an asphalt type waste solidification system to be consistent with current practice. (Cost Increase)

ACCOUNT 226 Othex Reactor Plant Equipment

The reactor water make-up system (except for a small quantity of pipe) was changed from safety class to non-safety class. This system does not have a safety class function. (Cost Decrease)

ACCOUNT 231 Turbine-Generator

Turbine-generator and moisture separator/reheater parameters were revised to agree with the revised heat balance. (No Cost Change)

Two lubricating oll transfex pumps were added to transfer oil between the storage tanks, reservoir and conditioner. (Cost Increase)

A second lubricating ofl storage tank was added so that one could be dedicated to clean ofl and one to dirty o1l. This makes it possible to transfer from the dirty oil tank to the clean oil tank through the conditioner, or receive a new batch of oil before the discarded oil has been picked up. (Cost Increase)

ACCOUNT 233 Condensing Systems

The condenser heat transfer surface was increased to match the revised heat balance. (Cost Increase)

The low pressure condensate booster pumps were deleted to simplify the condensate system. The total dynamic head (TDH) of the condensate pumps was increased to replace the TDH of the booster pumps. (Cost Decrease)

The quantity of condensate piping was increased to correct an error introduced in the Phase VII Update. (Cost Increase)

The low pressure condensate boostex pump foundations were deleted consistent with deleting the condensate booster pumps. (Cost Decrease) 
The condensate polishing system design parameters were revised to be consistent with the revised heat balance. (No Cost Change)

The condensate storage tank foundation (including shield wall) was reviewed resulting in a decrease in the quantity of structural commodities. (Cost Decrease)

\section{ACCOUNT 234 Feedheating System}

The feedwater heaters were resized to match the revised heat balance. (Cost Increase)

The design point of the steam generator feed pumps was revised to match the revised heat balance. (No Cost Change)

ACCOUNT $241 \quad$ Switchgear

Switchgear associated with the mechanical draft cooling tower fans was deleted since it was not needed with natural draft cooling towers. (Cost Decrease)

ACCOUNT 242 Station Service Equipment

Eight Non-Class 1E load center buses assoctated with the mechanical draft cooling towers were deleted due to the change to natural draft cooling towers. The two accounts containing this equipment were combined into one as a result of this deletion. (Cost Decrease)

Eight Non-Class IE load center transformers associated with the mechanical draft cooling towers were deleted due to the change to natural draft cooling towers. The two accounts containing this equipment were combined into one as a result of this deletion. (Cost Decrease)

The piping, valves, Instrumentation and controls associated with the dieselgenerator subsystems were reviewed and found to include several items which are furnished with the diesel-generator units. The separate listings in the plant equipment list for these items were deleted. (Cost Decrease)

ACCOUNT 245 Electrical Structures and Wiring Containers

The quantity of conduit and cable tray was reviewed considering current field data and the replacement of the mechanical draft cooling towers with natural draft cooling towers. For the median experience (ME) data model, a net increase resulted. (Cost Increase) For the best experfence (BE) data model, a net decrease resulted. (Cost Decrease)

ACCOUNT 246 Power and Control Wiring

The quantity of power wiring was reviewed considering current field data and replacement of the mechanical draft cooling towers with natural draft cooling towers. A net decrease resulted. (Cost Decrease) 
ACCOUNT 252 Air, Water and Steam Service Systems

The quantity of service water piping was increased to correct an error from the Phase VII Update. (Cost Increase)

Auxiliary steam piping sizes $1-1 / 2$ inches and 2 inches were changed from Schedule 80 to Schedule 40 since Schedule 40 was adequate. (Cost Decrease)

ACCOUNT 261 Main Condenser Heat Rejection System Structures

The cooling tower switchgear building was deleted because it is not required with natural draft cooling towers. (Cost Decrease)

ACCOUNT 262 Main Heat Rejection System Mechanical Equipment

The intake traveling water screens were changed to fish protection type to be consistent with current practice. (Cost Increase)

The configuration and quantity of concrete circulating watex pipe were revised In accordance with the change to natural draft cooling towers. The associated valves, excavation, backf $\$ 11$ and compacted sand bed were also revised. (Cost Decrease)

The bulk sulfuric acid storage tank was changed from carbon steel to lined carbon steel for better corrosion resistence. (Cost Increase)

The sulfuric acid feed piping was changed from carbon steel to stainless steel for better corrosion resistance. (Cost Increase)

The three mechanical draft cooling towers were replaced by two natural draft cooling towers, because natural draft is more economical at Middletown. This resulted in a reduction of five MWe of auxillary power. (Although the cost of the natural draft cooling towers exceeds that of mechanical draft cooling towers, the natural draft towers have lower operating costs which, at Middletown, more than compensate for the higher cost.) (Cost Increase)

The cooling tower basins were eliminated as a separate account because they are included in the scope of the natural draft cooling towers subcontract. (Cost Decrease)

The blowdown piping was changed from concrete to fiberglass reinforced plastic (FRP) because concrete is not readily available in the required size. (Cost Increase)

The circulating water flow was increased to be consistent with the revised heat balance. Pipe sizes, cooling towers, and circulating water pumps were reviewed, and any necessary changes made. Because the flow change was so small $(<0.2 \%)$, no cost change resulted. (No Cost Change) 


\subsubsection{EEDB Data Mode1 Number A5, Type LSPB, EEDB Phase VIII Update (1986)}

The LSPB data model was first incorporated into the EEDB during the Phase VII Update, when it replaced the LMFBR data model. At that time, it existed as system design descriptions and a three-digit code-of-accounts level of detail cost estimate only. No detalled EEDB LSPB equipment list had been developed. During this Phase VIII Update, the level of detail was expanded to the ninedigit code-of-accounts level of detail and the first EEDB LSPB equipment list was prepared. The level of detail included in the equipment list varies, but in most accounts it is fully developed to the nine-digit level of detail.

Technical changes to this data model made during the Phase VIII Update were mostly for the purpose of achieving greater consistency with EEDB ground rules and/or to make changes comparable to those made in the PWR data models. (A technical change is constdered to be one where a quantity or type of equipment or commodity is changed, or where a configuration or capacity of equipment is changed to reflect a structure or system design change.)

\section{Genera1}

The net station output has been decreased from 1320 MWe to 1311 MWe. This change results from a combination of: moving the design from the CoMO Idaho site to the EEDB Middletown site, which has different meteorological and other conditions; changing the multi-pressure condenser to a single pressure condenser, consistent with the other EEDB data models; and replacing the mechanical draft cooling towers with natural draft cooling towers. The impact of these changes on the various technical design features is described in the following paragraphs.

\section{ACCOUNT 211 Yardwork}

The temporary sanitary sewage system has been deleted from the system design description. It had not been included in the cost. (No Cost Change)

\section{ACCOUNT 231 Turbine-Generator}

The generator rating was changed from 1667 MVA to 1660 MVA consistent with the output at 2.5 in-HgA. (Negligible Cost Decrease)

A description of the steam packing exhauster was added to the system design description. (No Cost Change)

\section{ACCOUNT 233 Condensing Systems}

The condenser was changed from a multi-pressure type, based on a design wet bulb temperature of $63^{\circ} \mathrm{F}$, to a single pressure $(2.5 \mathrm{in}-\mathrm{HgA})$ type, based on a design wet bulb temperature of $68^{\circ} \mathrm{F}$, consistent with EEDB ground rules. (Cost Increase) 
The condenser gas removal system was revised to include three vacuum pumps instead of four vacuum pumps and air ejectors. This change is related to the change to a single pressure condenser. (Cost Decrease)

\section{ACCOUNT 241 Electric Switchgear}

Switchgear assoclated with the mechanical draft cooling tower fans was deleted since it was not needed with natural draft cooling towers. (Cost Decrease)

ACCOUNT 242 Station Service Equipment

Twelve Non-Class IE load center buses assoclated with the mechanical draft cooling towers were deleted due to the change to natural draft cooling towers. (Cost Decrease)

Twelve Non-Class IE load center transformers associated with the mechanical draft cooling towers were deleted due to the change to natural draft cooling towers. (Cost Decrease)

ACCOUNT 245 Electric Structures and Wixing Containers

Raceways associated with providing power for the cooling tower fans were deleted with the change to natural draft cooling towers. (Cost Decrease)

ACCOUNT 246 Power and Control Wiring

Wire and cable associated with providing power for the cooling tower fans were deleted with the change to natural draft cooling towers. (Cost Decrease)

ACCOUNT 261 Main Condenser Heat Rejection System Structures

The cooling tower switchgear building was deleted because it is not required whth natural draft cooling towers. (Cost Decrease)

ACCOUNT 262 Main Condenser Heat Rejection System Mechanical Equipment

The intake traveling water screens were changed to fish protection type to be consistent with current practice. (Cost Increase)

The configuration and quantity of concrete circulating water pipe were revised in accordance with the change to natural draft cooling towers. The associated valves, excavation, backf 111 and compacted sand bed were also revised. (Cost Decrease)

The three mechanical draft cooling towers were replaced by two natural draft cooling towers, because natural draft is more economical at Middletown. This resulted in a reduction of five MWe of auxiliary power. (Although the cost of the natural draft cooling towers exceeds that of mechanical draft cooling towers, the natural draft towers have lower operating costs which, at Middletown, more than compensate for the higher cost.) (Cost Increase) 
The cooling tower basins were eliminated as a separate account because they are included in the scope of the natural draft cooling towers subcontract. (Cost Decrease)

The circulating water flow was increased because of the lower temperature rise required by the single pressure condenser. This necessitated increases in pipe sizes and pump capacity. (Cost Increase)

The cooling towex design conditions were revised in accordance with the different circulating water flow and temperatures of the $2.5 \mathrm{in}-\mathrm{HgA}$ single pressure condenser. (Cost Decrease) 


\subsubsection{EEDB Data Model Number C5, Type HS5, EEDB Phase VIII Update (1986)}

Duxing the Phase VI Update, the HS5 technical data model was expanded from the three-digit to the nine-digit code-of-accounts level of detail, using the HS8 as a basis. For the Phase VII Update, the HS5 replaced the HS8 as the lead comparison plant data model. Consequently, a detalled review of the complete HS 5 technical data model was conducted to bring the HS 5 to a level of maturity comparable with that of the HS8. During the Phase VIII Update, the HS5 was again reviewed and further refinements were made. The following technical data model modifications resulted from this review.

ACCOUNT 225 Flue Gas Desulfurization Structures

The HVAC system for the Lime Feed Preparation and Storage Facility was revised to separate it from the Lime Preparation Electrical and Control Building HVAC. (Cost Increase)

\section{ACCOUNT 226 Desulfurization Equipment}

A blower and other equipment to pneumatically transfer lime from the maln storage silos to the sludge stabilization area silos were added. This equipment was erroneously omitted during the Phase VII Update. (Cost Increase)

Flue gas booster fans were added to overcome the pressure drop through the flue gas desulfurization system. The induced draft fans alone were not adequate. (Cost Increase)

\section{ACCOUNT 231 Turbine-Generatox}

The steam packing exhauster from Account 233 was added to the gland sealing system, because it is actually part of the gland sealing system and is supplied with the turbine-generator. (No Cost Change)

The description of the turbine gland sealing system was improved. (No Cost Change)

The description of the turbine lube oil storage and conditioning system was expanded. (No Cost Change)

An error on the heat balance was corrected. The correction was minor and resulted in no performance change. (No Cost Change)

\section{ACCOUNT 233 Condensing Systems}

The steam packing exhauster was moved to Account 231 as described above. (No Cost Change)

The low pressure condensate booster pumps were deleted to simplify the condensate system. The TDH of the condensate pumps was increased to replace the TDH of the booster pumps. (Cost Decrease) 
The low pressure condensate booster pump foundations were deleted consistent with deleting the condensate booster pumps. (Cost Decrease)

ACCOUNT 235 Other Turbine Plant Equipment

A separate demineralized water storage tank was added to be consistent with the PWR and because it is good practice.

ACCOUNT 241 Electric Switchgear

Switchgear assoclated with the mechanical draft cooling tower fans was deleted since it was not needed with natural draft cooling towers. (Cost Decrease)

ACCOUNT 242 Station Service Equipment

Four load center buses associated with the mechanical draft cooling towers were deleted due to the change to natural draft cooling towers. The two accounts contalning this equipment were combined into one as a result of this deletion. (Cost Decrease)

Four load center transformers associated with the mechanical draft cooling towers were deleted due to the change to natural draft cooling towers. The two accounts containing this equipment were combined into one as a result of this deletion. (Cost Decrease)

ACCOUNT 245 Electric Structures and Wiring Containers

Raceways assoctated with providing power for the cooling tower fans were deleted with the change to natural draft cooling towers. (Cost Decrease)

ACCOUNT 246 Power and Control Wiring

Wire and cable associated with providing power for the cooling tower fans was deleted with the change to natural draft cooling towers. (Cost Decrease)

ACCOUNT 261 Heat Rejection System Structures

The battery and switchgear room at the Intake Structure was eliminated from the system design description. It was not included in the cost. (No Cost Change)

The discharge structure was eliminated from the cost data model since it was previously deemed unnecessary and was replaced by a circulating water system blowdown discharge pipe in the system design description. (Cost Decrease)

ACCOUNT 262 Heat Rejection System Mechantcal Equipment

The circulating water blowdown pipe size was changed from ten inches to eight inches because the discharge velocity was too low in a ten inch pipe. (Cost Decrease) 
The intake traveling water screens were changed to fish protection type to be consistent with current practice. (Cost Increase)

The mechanical draft cooling tower was replaced by a natural draft cooling tower because natural draft is more economical at Middletown. This resulted in a reduction of two MWe of auxiliary powex. (Although the cost of the natural draft cooling towers exceeds that of mechanical draft cooling towers, the natural draft towers have lower operating costs which, at Middletown, more than compensate for the higher cost.) (Cost Increase)

The cooling tower basin was eliminated as a separate account because it is included in the scope of the natural draft cooling tower subcontract. (Cost Decrease) 
TABLE 4-1

ENERGY ECONOMIC DATA BASE

PHASE VIII UPDATE (1986)

DIRECT COMMODITY/EQUIPMENT/LABOR SUMMARY FOR NUCLEAR AND COMPARISON POWER GENERATING STATIONS FOR 1986

\section{Data Model/Rating (MWe)}

\section{Commodity/Equipment}

\section{Excavation}

Formwork

Reinforcing, Embedded and

Structural Steel

Structural Concrete

BOP Pumps ( $1000 \mathrm{HP}$ and up) (c)

Piping (d)

Raceways

$\stackrel{t}{\infty}$

Wire and Cable

Steam Supply System

Coal and Ash Handling

Precipitator and FGD System $(f)$

Turbine-Generator

Heat Exchangers ( $g$ )

Instrumentation and Control

Craft Manhours (h)

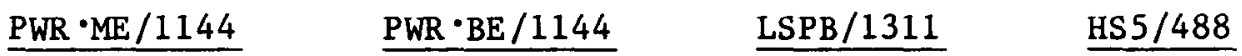

$\underline{\text { Unit }}(\mathrm{a})$

Commodities (Quantity $\times 10^{3(\mathrm{~b})}$ )

$\begin{array}{lrrrr}\text { CY } & 573 & 470 & 361 & 131 \\ \text { SF } & 2,132 & 1,885 & 2,118 & 633 \\ \text { TN } & 38 & 29 & 42 & 19 \\ & & & & \\ \text { CY } & 161 & 133 & 182 & 57 \\ \text { HP } & 67 & 67 & 89 & 25 \\ \text { LB } & 11,074 & 8,957 & 9,129 & 3,708 \\ \text { LF } & 872 & 672 & 849 & 442 \\ \text { LF } & 6,272 & 4,979 & 4,655 & 2,660\end{array}$

Equipment $\left(\$ / \mathrm{kWe}^{(\mathrm{e})}\right)$

$\begin{array}{lrrrr}\text { LT } & 164 & 164 & 480 & 144 \\ \mathrm{LT} & \text { N/A } & \text { N/A } & \text { N/A } & 64 \\ \text { LT } & \text { N/A } & \text { N/A } & \text { N/A } & 131 \\ \text { LT } & 108 & 108 & 105 & 100 \\ \text { LT } & 46 & 44 & 39 & 40 \\ \text { LT } & 32 & 29 & 32 & 19\end{array}$

Manhours (Quantity $\times 10^{3(b)}$ )

$\begin{array}{lllll}\mathrm{N} / \mathrm{A} & 30,481 & 16,623 & 21,414 & 6,752\end{array}$

$16,623 \quad 21,414$

, 752

(a) $\mathrm{CY}=$ Cubic Yards; $\mathrm{HP}=$ Horsepower; $\mathrm{LB}=$ Pounds; LF = Linear Feet; LT = Lot; SF = Square Feet; TN = Tons

(b) Rounded off to the Nearest 1,000 Units

(c) Includes Steam Generator or Boiler Feed Pumps; Does Not Include NSSS Primary (or Intermediate) System Pumps

(d) Includes Carbon Steel and Stainless Steel Piping for PWR $\cdot M E$ and PWR·BE, Plus Chrome-Moly Piping for LSPB and HS5

(e) Installed Costs in January 1, 1986 Constant Dollars

(f) Flue Gas Desulfurization System: Does Not Include Comodities for Structures and Equipment Foundations; Draft Booster Fans; Piping; or HVAC, I\&C, and Electrical Equipment

(g) Includes Cooling Towers and Condensers

(h) Includes Indirect Craft Manhours 
TABLE $4-2$

ENERGY ECONOMIC DATA BASE

PHASE VII UPDATE (1984)

II: I

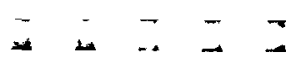

$-\pi+7$
$4-4$

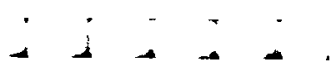

DIRECT COMMODITY/EQUIPMENT/LABOR SUMMARY FOR NUCLEAR AND COMPARISON POWER GENERATING STATIONS FOR 1984

Data Mode1/Rating (MWe)
Commodity/Equipment
Excavation
Formwork
Reinforcing, Embedded and
Structural Stee1
Structural Concrete
Bop Pumps (1000 HP and up)(c)
Piplag(d)
Raceways
Wire and Cable
$\hat{i} \quad$

Steam Supply System

Coal and Ash Handling

Precipitator and FGD System(f)

Turbine-Generator

Heat Exchangers $(g)$

Instrumentation and Control

\begin{tabular}{l} 
Undt (a) \\
\hline CY \\
SF \\
TN \\
CY \\
UP \\
LB \\
LF \\
LF
\end{tabular}

PWR $\cdot M E / 1139$

LSPB / 1320

HS5/486 Commodities (Quantity $\times 10^{3(b)}$ )

\begin{tabular}{rrrr}
\hline 645 & 541 & 522 & 155 \\
2,158 & 1,698 & 1,764 & 645 \\
39 & 30 & 40 & 19 \\
& & & \\
168 & 139 & 199 & 59 \\
66 & 66 & 39 & 25 \\
10,781 & 8,730 & N/Av & 3,708 \\
725 & 676 & 520 & 445 \\
6,276 & 5,416 & 4,675 & 2,661 \\
& & & \\
& Equipment $(\mathrm{S} / \mathrm{kWe}(\mathrm{e}))$ & \\
154 & & & \\
N/A & 154 & N/A & 62 \\
N/A & N/A & N/A & 128 \\
101 & N/A & 93 & 94 \\
39 & 101 & N/Av & 30 \\
30 & 38 & N/Av & 17
\end{tabular}

Manhours (Quantity $\times 10^{3(b)}$ )

Craft Manhours (h)

N/A

15,572

11,532

6,640

(a) $\mathrm{CY}=$ Cubic Yards, $\mathrm{HP}=$ Horsepower; LB = Pounds; LF = Linear Feet; LT = Lot; SF = Square Feet; TN $=$ Tons

(b) Rounded off to the Nearest 1,000 Units

(c) Includes Steam Generator or Boiler Feed Pumps; Does Not Include NSSS Primary (or Intermediate) System Pumps

(d) Includes Carbon Steel and Stainless Steel Piplng for PWR -ME and PWR-BE, Plus Chrome-Moly Piping for LSPB and HS5

(e) Installed Costs in January 1, 1984 Constant Dollars

(f) Flue Gas Desulfurization System: Does Not Include Commodities for Structures and Equipment Foundations; Piping; or HVAC, I\&C, and Electrical Equipment

(g) Includes Cooling Towers and Condensers $\mathrm{N} / \mathrm{A}=$ Not Applicable (h) Includes Indirect Craft Manhours N/AV = Not Avallable 
TABLE $4-4$

ENERGY ECONOMIC DATA BASE

PHASE VIII UPDATE

DIRECT COMMODITY AND CRAFT MANHOUR SUMMARY
1144 MWe PRESSURIZED WATER REACTOR NUCLEAR POWER GENERATING STATION - BAERT REACTOR NUCLEAR POWER
- EXPENCE (PWR'BE)

NUCLEAR PLANT QUANTITIES

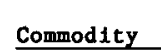

\section{Excavation (Rock/Earth)}

F11l (Rock/Earth)

Formwork

Reinforcing Steel

Structural Concrete

Concrete Fill

Structural Steel

Spectal Steel Liner

Carbon Steel Piping (NS)

Stainless Steel Piping (NS)

Carbon Steel Piping (NNS)

Stalnless Steel Piplng (NNS)

Valves

Fire Protection

BOP Pumps (1000 HP \& above)(d)

Heat Exchangers $(\mathrm{e})$

Turbine Generator

Instrumentation and Control

heat., Ventilating, \& Alr Cond. (f)

Lighting Service Power

Duct Runs and Wire Containers

Wre and Cable

Electrical Balance of Plant

Nuclear Steam Supply Systeu

\begin{tabular}{c} 
\\
Installed \\
Cost/Unit (b) \\
\hline 13.21 \\
3.81 \\
16.57 \\
$1,449.57$ \\
106.58 \\
84.89 \\
$9,705.74$ \\
$2,397.71$ \\
$22.25(\mathrm{c})$ \\
11.31 \\
36.71 \\
8.13 \\
24.65 \\
$10.55(\mathrm{c})$ \\
$5.98(\mathrm{c})$ \\
115.39 \\
$44.49(\mathrm{c})$ \\
$107.72(\mathrm{c})$ \\
$28.91(\mathrm{c})$ \\
$7.16(\mathrm{c})$ \\
$4.50(\mathrm{c})$ \\
28.95 \\
3.54 \\
$39.20(\mathrm{c})$ \\
$163.93(\mathrm{c})$ \\
$100.78(\mathrm{c})$
\end{tabular}

NUCLEAR PLANT MANHOURS

\begin{tabular}{|c|c|c|}
\hline Craft & Manho & $\begin{array}{l}\text { Cost } x \\
10^{3(b)} \\
\end{array}$ \\
\hline Boller Makers & 670,414 & 15,621 \\
\hline Bricl & 263, & 5,012 \\
\hline Carpenters & $1,356,253$ & 27,925 \\
\hline Electricians & $2,207,985$ & 52,241 \\
\hline Iron & 0,765 & 28,861 \\
\hline Laborers & $1,783,337$ & 29,282 \\
\hline Millwrights & 195,232 & 4,598 \\
\hline 8 Engineers & 906 & 19,402 \\
\hline Painters & 302,353 & 5,521 \\
\hline Pipe & $2,952,599$ & 72,575 \\
\hline Sheet Metal Worke & 18,552 & 433 \\
\hline Teamsters & 151,152 & 2,438 \\
\hline All Others & 149,559 & 3,694 \\
\hline atal Craft Labo & 188,515 & \\
\hline
\end{tabular}

(a) CY - CubIC Yards; HP = Horsepower; LB = Pounds; LF = Linear Feet; LT = Lot; SF = Square Feet; TN = Tons

(b) Data In January 1, 1986 Constant Dollars

(c) Cost per Unit is in Dollars per Kilowatt - Electrical ( $\$ / \mathrm{kWe}$ )

(NNS) $=$ Non-Nuclear Safety Grade

(e) Includes Steam Generator Feed Pumps; Does

(f) Includes Ductwork and All HVAC Equipment

(g) Does Not Include Indirect Costs

$r i$
$r i$
$r$
$i$,

$r$
$r$
$r$
$r 1$

$r$

11

$m$

11
11
11
11
.1

1
+1
1
1
1




\begin{tabular}{|c|c|c|c|c|c|c|c|c|c|c|c|}
\hline 1 & $*:$ & $\approx$ & $\therefore ;$ & $\cdots$ &. & $\cdots$ & . & $\ldots$ & $\cdots$ & . & \\
\hline$c i$ & $F$ & 3 & 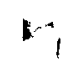 & 1 & E: & 11 & k 1 & $\omega$ & wa & $E 1$ & \\
\hline$\theta j$ & $r$ & E: & $E ;$ & L. & 11 & b. & $t$ & $\omega$ & $m$ & 11 & 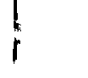 \\
\hline and & Fis & F: & $5:$ & $b$ & k: & $w$ & $: 1$ & 4 & $M$ & A. 1 & \\
\hline$\rightarrow$ & o & 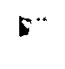 & $r$ & $b$ & 14 & $w$ & 11 & id & $\omega$ & 11 & s. \\
\hline$\Leftrightarrow$ & $k_{1}$ & $\mathbf{E}$ & 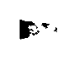 & 1 & Lid & $u$ & $\omega$ & $\omega$ & 14 & t & \\
\hline
\end{tabular}

Effective Date $1 / 1 / 86$

TABLE 4-5

ENERGY ECONOMIC DATA BASE

PHASE VIII UPDATE

DIRECT COMMODITY AND CRAFT MANHOUR SUMMARY

1311 MWe LARGE SCALE PROTOTYPE BREEDER NUCLEAR POWER GENERATING STATION (LSPB)

NUCLEAR PLANT QUANTITIES

Commodity
Excavation (Rock/Earth)
Fill (Rock/Earth)
Formwork
Reinforcing Steel
Structural Concrete
Concrete Fill
Embedded Steel
Structural Steel
Special Steel Liners
Carbon Steel Piping (NS)
Stainless Steel Piping (NS)
Carbon Steel Piping (NNS)
Stainless Steel P1ping (NNS)
Chrome-Moly Piping (NNS)
Valves
Fire Protection
BOP Pump (1000 HP \& above)(d)
Heat Exchangers(e)
Turbine Generator
Instrumentation and Contro1
Heat., Ventilating, \& Air Cond.(f)
Lighting \& Service Power
Duct Runs and Wire Containers
Wire and Cable
Electrical Balance of Plant
Nuclear Steam Supply System
All Others(g)

\begin{tabular}{|c|c|c|}
\hline Unit $(a)$ & Quantity & $\begin{array}{l}\text { Installed } \\
\text { Cost/Unit (b) }\end{array}$ \\
\hline CY & 361,423 & 7.63 \\
\hline$C Y$ & 236,976 & 4.93 \\
\hline $\mathrm{SF}$ & $2,118,003$ & 14.72 \\
\hline TN & 21,999 & $1,417.22$ \\
\hline $\mathrm{CY}$ & 182,383 & 109.03 \\
\hline CY & 96,169 & 84.89 \\
\hline TN & 1,709 & $9,702.90$ \\
\hline TN & 18,499 & $2,397.71$ \\
\hline $\mathbf{L T}$ & - & $28.93(c)$ \\
\hline$L B$ & 177,895 & 18.93 \\
\hline LB & 400,731 & 31.73 \\
\hline LB & $7,036,303$ & 8.14 \\
\hline LB & 177,260 & 24.60 \\
\hline LB & $1,336,396$ & 16.54 \\
\hline $\mathbf{L T}$ & - & $13.24(c)$ \\
\hline LT & - & $4.12(c)$ \\
\hline $\mathrm{HP}$ & 89,230 & 116.01 \\
\hline$L T$ & 8 & 38.90 (c) \\
\hline LT & - & 104.66 (c) \\
\hline LT & - & $32.34(c)$ \\
\hline$L T$ & - & 36.98 (c) \\
\hline $\mathbf{L T}$ & - & $6.38(c)$ \\
\hline $\mathbf{L F}$ & 849,200 & 27.77 \\
\hline LF & $4,655,000$ & 3.84 \\
\hline LT & , & $43.36(c)$ \\
\hline$L T$ & - & $479.85(c)$ \\
\hline $\mathrm{L} T \mathrm{~T}$ & - & $114.58(c)$ \\
\hline
\end{tabular}

NUCLEAR PLANT MANHOURS

\begin{tabular}{|c|c|c|}
\hline Craft & Manhours & $\begin{array}{l}\text { Cost } x \\
10^{3}(b) \\
\end{array}$ \\
\hline Boller Makers & 588,540 & 13,713 \\
\hline Bricklayers & 332,612 & 6,323 \\
\hline Carpenters & $1,473,315$ & 30,336 \\
\hline Electriclans & $3,084,336$ & 72,975 \\
\hline Ironworkers & $2,079,134$ & 48,756 \\
\hline Laborers & $1,971,887$ & 32,378 \\
\hline Millwrights & 291,992 & 6,876 \\
\hline Operating Engineers & $1,174,155$ & 25,127 \\
\hline Painters & 391,775 & 7,154 \\
\hline Pipe Fitters & $3,765,757$ & 92,562 \\
\hline Sheet Metal Workers & 100,000 & 2,335 \\
\hline Teamsters & 222,710 & 3,592 \\
\hline All others & 337,420 & 8,379 \\
\hline Cotal Craft Labor $(\mathrm{h})$ & 633 & 507 \\
\hline
\end{tabular}

(a) CY = Cubic Yards; HP = Horsepower; LB = Pounds; LF = Linear Feet; LT = Lot; SF = Square Feet; TN = Tons

(b) Data 1n January 1, 1986 Constant Dollars

(c) Cost per Unit is In Dollars per Kilowatt - Electrical ( $\$ / \mathrm{kWe}$ )

(d) Includes Steam Generator Feed Pumps; Does Not Include NSSS Primary or Intermediate System Pumps

(e) Includes Cooling Towers and Condenser

(f) Includes Ductwork and All HVAC Equipment; Excludes Estimated Piping Quantities/Cost

$\begin{array}{ll}\text { (g) Does Not Include Indirect Costs } & \text { (NNS) }=\text { Non-Nuclear Safety Grade } \\ \text { (h) Does Not Include Indirect Manhours } & \text { (NS) }=\text { Nuclear Safety Grade }\end{array}$

(NS) = Nuclear Safety Grade 
5

$r:$

Fis

v.

$*$

\section{i}

i

i

I ;

$5:$

$r$

5

1
L.
b.
1.

$1:$
11
$1:$
11

I
I
1
L.d

Effective Date $1 / 1 / 86$

TABLE 4-6

ENERGY ECONOMIC DATA BASE

PHASE VIII UPDATE

DIRECT COMMODITY AND CRAFT MANHOUR SUMMARY

488 MWe HIGH SULFUR COAL-FIRED POWER GENERATING STATION (HS5)

COMPARISON COAL PLANT QUANTITIES

Commodity
Excavation (Rock/Earth)
Fill (Rock/Earth)
Formwork
Reinforcing Steel
Structural Concrete
Concrete F1l1
Embedded Steel
Structural Steel
Carbon Steel P1ping
Stainless Steel Piping
Chrome-Moly Piping
Valves
Fire Protection
Pumps (1000 HP \& above)(d)
Heat Exchangers(e)
Turbine Generator
Coal Handling(f)
Electrostatic Precipitator
FGD System(g)
Ash Handling
Instrumentation and Control
Heat. Vent1lating, \& Alr Cond. (h)
Lighting \& Service Power
Duct Runs \& Wire Containers
Wire and Cable
Electrical Balance of Plant
Fossll Steam Supply System
All Others(i)

COMPARISON COAL PLANT MANHOURS

\begin{tabular}{|c|c|c|}
\hline Unit & Quantity & $\begin{array}{l}\text { Installed } \\
\text { Cost/Unit (b) }\end{array}$ \\
\hline $\mathrm{CY}$ & 131,241 & 7.46 \\
\hline CY & 111,081 & 21.61 \\
\hline SF & 633,137 & 10.60 \\
\hline TN & 3,551 & $1,172.66$ \\
\hline CY & 56,617 & 85.05 \\
\hline CY & 4,000 & 70.69 \\
\hline TN & 251 & $7,177.15$ \\
\hline $\mathrm{TN}$ & 14,923 & $1,939.02$ \\
\hline $\mathrm{LB}$ & $2,659,740$ & 8.14 \\
\hline LB & 1,560 & 24.74 \\
\hline LB & $1,046,400$ & 11.10 \\
\hline LT & - & $5.77(\mathrm{c})$ \\
\hline LT & - & $5.64(\mathrm{c})$ \\
\hline HP & 25,000 & 147.57 \\
\hline LT & - & $39.98^{(c)}$ \\
\hline LT & - & 100.45 (c) \\
\hline LT & - & $51.95(c)$ \\
\hline LT & - & 14.79 (c) \\
\hline $\mathrm{LT}$ & - & $116.22(c)$ \\
\hline LT & - & 12.01 (c) \\
\hline LT & - & $18.68^{(c)}$ \\
\hline LT & 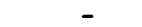 & $8.49(c)$ \\
\hline LT & - & $8.49(c)$ \\
\hline LF & 442,400 & 22.87 \\
\hline LF & $2,660,000$ & 3.22 \\
\hline LT & - & 40.56 (c) \\
\hline LT & - & 144.26 (c) \\
\hline & & \\
\hline
\end{tabular}

\begin{tabular}{|c|c|c|}
\hline Craft & an! & $\begin{array}{l}\cos t{ }^{x} \\
10^{3}(\mathrm{~b}) \\
\end{array}$ \\
\hline oiler Makers & 636,073 & 14,820 \\
\hline Bric & 99,630 & 1,894 \\
\hline Carpenters & 317,139 & 6,530 \\
\hline Electricians & $, 020,739$ & 24,151 \\
\hline Ironworkers & 482,831 & 11,322 \\
\hline Labo & 681 & 11,193 \\
\hline Millwrights & 157,353 & 3,706 \\
\hline g Engine & 389 , & 8,340 \\
\hline Painters & 156,069 & 2,850 \\
\hline Pipe Fitters & $1,747,626$ & 42,957 \\
\hline Sheet Metal Workers & 91,455 & 2,135 \\
\hline Teamsters & 73,136 & 1,180 \\
\hline All Others & 74,753 & 1,838 \\
\hline TOTAL CRAFT LABOR & 928,200 & 91 \\
\hline
\end{tabular}

(a) CY = Cubic Yards; HP = Horsepower; LB $=$ Pounds; LF $=$ Linear Feet; LT $=$ Lot; SF = Square Feet; TN $=$ Tons

(b) Data in January 1, 1986 Constant Dollars 4 (c)

(d) Includes Boiler Feed Pumps

(e) Includes Cooling Towers and Condensers

(f) Does Not Include Ignition 011 System

(g) Flue Gas Desulfurization System: Does Not Include Commodities for Structures and Equipment Foundations; Draft Booster Fans; Piping; or HVAC, I\&C, and Electrical Equipment

h) Includes Ductwork and All HVAC Equipment; Excludes Flue Gas Ductwork

(1) Does Not Include Indirect Costs

(j) Does Not Include Indirect Manhours 
ENERGY ECONOMIC DATA BASE PHASE VIII UPDATE

DIVISION I REGULATORY GUIDES ISSUED OR REVISED BETWEEN JANUARY I, 1984 AND JANUARY 1,1986 AND THEIR PROJECTED IMPACT ON BEDB PHASE VIII UPDATE TECHNICAL FEATURES AND COST ESTIMATES

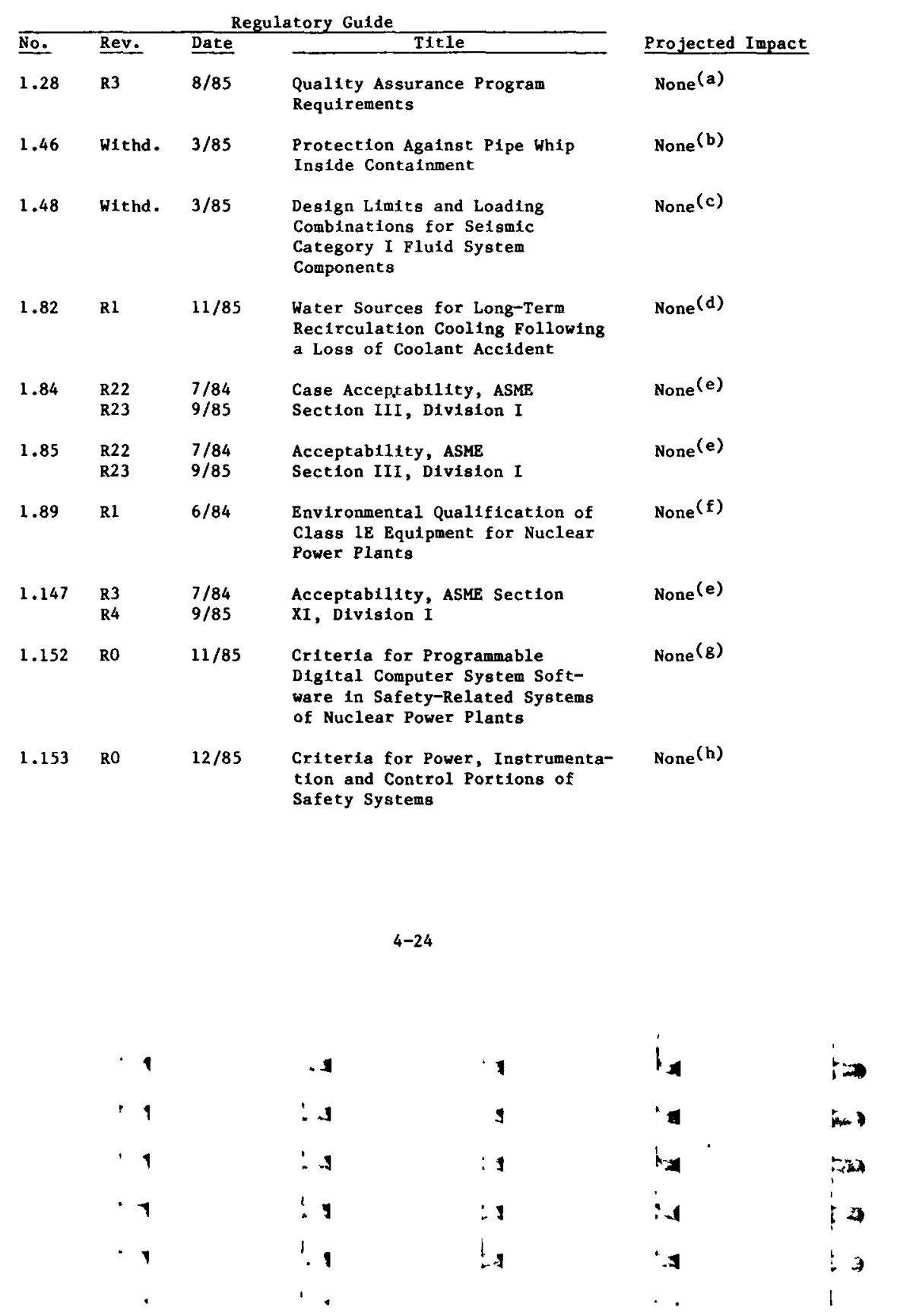


TABLE 4-7

ENERGY ECONOMIC DATA BASE

PHASE VIII UPDATE

DIVISION I REGULATORY GUIDES ISSUED OR REVISED BETWEEN

JANUARY 1, 1984 AND JANUARY 1, 1986 AND THE IR PROJECTED

IMPACT ON EEDB PHASE VIII UPDATE TECHNICAL

FEATURES AND COST ESTIMATES

NOTES

(a) This revised regulatory guide invokes the 1983 ANSI/ASME Standard NQA-1 and Addenda as being acceptable to the NRC staff as a basis for complying with Appendix B to 10CFR50 with exceptions. Since the standard is representative of existing industry practice, there is no impact on the Phase VIII Update.

(b) This regulatory guide was withdrawn because Standard Review Plan 3.6.2 (7/81 Revision) provides more current information. Therefore, this action has no impact for the 1986 Update because no new obligations are imposed, nor are any old obligations rescinded.

(c) This regulatory guide was withdrawn because Standard Review P1an 3.9.3 (7/81 Revision) provides more current information. Therefore, this action has no impact for the 1986 Update because no new obligations are imposed, nor are any old obligations rescinded.

(d) This regulatory guide revision addresses the resolution of Unresolved Safety Issue A-43 "Containment Emergency Sump Performance" as given in NUREG $0869, \mathrm{Rl}, 10 / 85$. It is not clear what the cost impact will be on completed or nearly completed plants at this time. In any event, its effective date and the EEDB regulatory and cost dates are so close, that there is essentially no impact for the Phase VIII Update.

(e) These regulatory guide revisions indicate acceptability of individual ASME code cases and, therefore, have no genexic impact on the Phase VIII Update.

(f) This regulatory guide revision invokes the 1974 revision of IEEE Standard 323 as being acceptable for meeting the Commission's requirements for electric equipment qualification with some exceptions. Since the standard is representative of existing industry practice, there is no impact on the Phase VIII Update.

(g) This new regulatory guide invokes the 1982 ANSI/IEEE-ANS Standard 7.4.3.2 as establishing a methodology acceptable to the NRC staff relative to software used in safety-related systems. Since the standard is representative of existing industry practice, there is no impact on the Phase VIII Update. 
TABLE 4-7

ENERGY ECONOMIC DATA BASE

PHASE VIII UPDATE

DIVISION I REGULATORY GUIDES ISSUED OR REVISED BETWEEN

JANUARY 1, 1984 AND JANUARY 1, 1986 AND THEIR PROJECTED

IMPACT ON EEDB PHASE VIII UPDATE TECHNICAL

FEATURES AND COST ESTIMATES

NOTES (cont'd)

(h) This new regulatory guide invokes the 1980 revision of IEEE Standard 603 as providing an acceptable method for meeting the Commission's requirements on the design, reliability, qualification and testability of the power, instrumentation and control portions of safety systems with some exceptions. Since the standard is representative of existing industry practice, there is no impact on the Phase VIII Update. 
TABLE 4-8

ENERGY ECONOMIC DATA BASE

PHASE VIII UPDATE

COOLING METHODS UTILIZED IN U.S. NUCLEAR POWER PLANTS

\begin{tabular}{l} 
Geographical \\
Regions (See \\
Sheet 2 of 2 ) Units \\
\hline
\end{tabular}

Cooling Type

Cooling Tower

Once Thru Nat. Draft Mech. Draft Helper(a) Other

\begin{tabular}{|c|c|c|c|c|c|c|}
\hline 1 (NE) & 6 & 6 & 0 & 0 & 0 & 0 \\
\hline 2 (NE) & 8 & 7 & 1 & 0 & 0 & 0 \\
\hline $3(\mathrm{NE})$ & 16 & 6 & 6 & 0 & 2 & 4 \\
\hline 4 & 37 & 5 & 14 & 6 & 5 & 12 \\
\hline 5 & 23 & 5 & 6 & 5 & 2 & 7 \\
\hline $6(S W)$ & 9 & 2 & 1 & 2 & 0 & 4 \\
\hline 7 & 5 & 2 & 1 & 1 & 0 & 1 \\
\hline 8 & 1 & 0 & 0 & 1 & 0 & 0 \\
\hline 9 (SW) & 8 & 4 & 1 & 3 & 0 & 0 \\
\hline 10 & 4 & 0 & 2 & 2 & $\underline{0}$ & 0 \\
\hline TOTAL & $117^{(b)}$ & 37 & 32 & 20 & - & 28 \\
\hline
\end{tabular}

\footnotetext{
(a) In conjunction with another cooling system. Includes both mechanical and natural draft.

(b) Excludes units that went into commercial operation prior to the Federal Water Pollution Control Act of October, 1972. Includes units that have been cancelled or indefinitely deferred.
}

Source: Commercial Nuclear Power Plants, Edition No. 17 by NUS Corporation. 
TABLE $4-8$

\section{ENERGY ECONOMIC DATA BASE \\ PHASE VIII UPDATE}

COOLING METHODS UTILIZED IN U.S. NUCLEAR POWER PLANTS IDENTIFICATION OF STATES IN TEN GEOGRAPHICAL REGIONS

IN THE CONTIGUOUS UNITED STATES

\begin{tabular}{ll} 
Region & \multicolumn{1}{c}{ States } \\
1 & $\mathrm{CT}, \mathrm{MA}, \mathrm{ME}, \mathrm{NH}, \mathrm{RI}, \mathrm{VT}$ \\
3 & $\mathrm{NJ}, \mathrm{NY}$ \\
4 & $\mathrm{DE}, \mathrm{MD}, \mathrm{PA}, \mathrm{VA}, \mathrm{WV}$ \\
5 & $\mathrm{AL}, \mathrm{FL}, \mathrm{GA}, \mathrm{KY}, \mathrm{MS}, \mathrm{NC}, \mathrm{SC}, \mathrm{TN}$ \\
6 & $\mathrm{IL}, \mathrm{IN}, \mathrm{MI}, \mathrm{MN}, \mathrm{OH}, \mathrm{WI}$ \\
7 & $\mathrm{AR}, \mathrm{LA}, \mathrm{OK}, \mathrm{NM}, \mathrm{TX}$ \\
8 & $\mathrm{IA,KS}, \mathrm{MO}, \mathrm{NE}$ \\
9 & $\mathrm{ID}, \mathrm{OR}, \mathrm{WA}$
\end{tabular}


TABLE 4-9

ENERGY ECONOMIC DATA BASE

PHASE VIII UPDATE

NATURAL DRAFT VS. ROUND MECHANICAL DRAFT COOLING TOWERS FOR THE PWR ON THE MIDDLETOWN SITE

$\underline{\text { Natural Draft }}$

Number of Towers

Piping Complexity

Capital Cost (1986 Constant Dollars $\left.\times 10^{6}\right)$

Cooling Towers (CT)

Cooling Tower Basins

CT Switchgear Butlding

CT Switchgear

Power Wiring

Total

Delta
2

Base

29.2

Incl. w/Towers

N/A

N/A

Negligible

29.2

Base
Round Mechanical Draft

3

Higher

24.5

3.6

0.4

1.1

$\underline{0.6}$

30.2

1.0

Auxtliary Power (kWe)

Fans

Circulating Water Pumps

Tota1

Delta
0

6,290

6,290

Base
5,375

$\underline{5,190}$

10,565

4,275 


\section{SECTION 5}

\subsection{COST UPDATE FOR SINGLE UNIT NUCLEAR AND COMPARISON POWER PLANTS}

As stated in Section 4, the Phase VIII Update of the Base Construction Costs only in the EEDB was accomplished in two separate steps. Section 4 presented the results of the first step, which was the evaluation and adjustment or extension of the technical data models selected for the update. This section of the report presents the results of the second step, which was the adjustment of the cost data models to accommodate the technical data model revisions and to reflect the effects of cost escalation since the last update.

\subsection{COST DATA MODEL UPDATE PROCEDURE}

A specific cost update procedure was developed for the EEDB during the Phase I Update in 1978, which includes both the technical and cost update steps. This procedure is described in Section 6 of the EEDB Program Reference Book. 8 The Phase VIII Update utilized a Level 2 (nine-digit code-of-accounts leve1 of detail) Cost Update for the PWR ME, PWR BE, LSPB and HS5, in conjunction with their Level 2B Technical Updates discussed in Section 4.

In the Phase VIII Update, commodity and equipment costs were updated to January 1,1986 , in accordance with the applicable specifications for Level 2 Cost Updates. As discussed in Section 4.1, a Level 2 update normally examines the major cost drivers. In the Phase VIII Update, costs for many systems/ equipment outside of the cost driver envelope were estimated in order to assure current cost credibility for the PWR, LSPB and HS5. This was particularly true for the LSPB, in order to support the extension of detail and conformance effort discussed in Section 4.3.2.3. Table 5-1 identifies the major equipment in the PWR, LSPB and HS5 for which estimating quotations were recelved. It also identifies major equipment for which estimates were made based on estimating quotations for similar equipment that were obtained for the Phase VII (1984) Update. In the case of the LSPB, the estimating quotations are based on the CoMO LSPB data where it was available and appeared to be consistent with EEDB data. Where quotations included installation, manhours and installation costs were provided to preserve the integrity of the EEDB factory equipment and labor costs. Installation costs were deducted from quoted installed equipment costs prior to inclusion in the data base. Quoted manhours and "Middletown" labor rates were then used to develop the associated EEDB labor cost.

Unit costs were obtained for structural (e.g., concrete, formwork, structural steel) and piping commodities utilizing national indices, United Engineers' power plant experience and field data from company private sources. Labor rates were updated by craft to January 1, 1986. Equipment costs that were not updated by estimating quotations were escalated to January 1,1986 by standard percentages, based on United Engineers' experience and national indices. Generally, the unweighted average increase for all industrial commodities, due to escalation between January 1, 1984 and January 1, 1986, was approximately one percent for equipment and six percent for labor. 
As mentioned in Section 4.1, a major task for the Phase VIII Update was the extension of the LSPB from the three-digit to the nine-digit code-of-accounts level of detail. A corresponding cost data model was developed and was incorporated into the data base. It was also noted that the BWR $M E$ technical data model was updated under a separate contract. Corresponding cost data models for a BWR $\cdot M E$ with a Mark III and a Mark II containment were also developed under that contract. The results of this work are given in the Phase VIII Update (1986) Report - BWR Supplement.12 The BWR ME cost data model update was done in conjunction with and benefitted from the results of the Phase VIII PWR -ME cost data model update. Consequently, the updated BWR ME cost data models and estimates are consistent with and comparable to the EEDB data models updated in the Phase VIII Update.

\subsection{COST SUMMARY}

Costs were prepared for the EEDB as base construction costs, which equal the direct plus indirect costs. EEDB base construction costs include only those cost elements described in the EEDB Program Reference Book. They specifically exclude owner's costs, contingencies, escalation and allowance for funds used during construction. These and other excluded preferential and discretionary items are listed in Table 5-2. Direct, indirect and base construction costs are summarized in Tables $2-3,2-4$ and 2-5.

During the Phase VI Update, a new PWR technical/cost data model was created which was called the "PWR for the 1990's." This data model had reduced quantities of materials, improved labor productivities and reduced indirect costs, which reflected the best cost experlence for nuclear power plants then under construction. At the same time, the old PWR technical/cost data model, based on median nuclear power plant cost experience, was renamed the "PWR for the 1980's." The designation "PWR for the 1990's" implied that the improved construction practices plus nuclear regulatory and licensing reforms anticipated during the next decade would result in future plants having average costs that were comparable to the costs for the current best experience plants. Since the terms "PWR for the 1980's" (median cost experience) and "PWR for the 1990's" (best cost experience) led to confusion in interpreting the results of the Phase VI Update, these data models were redesignated PWR-ME and PWR·BE respectively in the Phase VII and later updates.

Events observed during the Phase VIII Update seem to indicate that the Light Water Reactor Nuclear Power Generating Station (LWR) for the 1990 's and beyond will be based on current technology with advanced design features. The advanced design features will most 11kely be in the direction of plant simplification that improves safety, maintainability, operability, cost and construction schedule. Consequently, the EEDB best experience data models ( $P W R \cdot B E$ and $B W R \cdot B E$ ) will become technical and cost benchmarks against which the LWR's of the 1990's may be compared. Section 6 provides a report on the Phase VIII Update review of potential nuclear power plant designs for the $1990^{\prime}$ 's and beyond. 


\subsection{DETAILED COSTS}

Cost results of the Phase VIII Update are presented for each technical data model at the two-digit and three-digit code-of-accounts level of detail in Tables 5-3 through 5-6 as indicated below. The first sheet of each table is a cost and manhours tabulation at the two-digit code-of-accounts (plant account) level of detail. The following four sheets comprise the cost and manhour tabulations at the three-digit code-of-accounts (structure/system account) level of detail. Additional detail for the PWR $\cdot M E, P W R \cdot B E, L S P B$ and HS5 to the nine-digit code-of-accounts (commodity/component account) level of detail, is available in the EEDB Backup Data File.

\begin{tabular}{|c|c|c|c|}
\hline $\begin{array}{c}\text { Nuclear } \\
\text { Plant Data } \\
\text { Models } \\
\end{array}$ & $\begin{array}{l}\text { Table } \\
\text { Number }\end{array}$ & $\begin{array}{c}\text { Comparison } \\
\text { Plant Data } \\
\text { Mode1 } \\
\end{array}$ & $\begin{array}{l}\text { Table } \\
\text { Number }\end{array}$ \\
\hline $\mathrm{PWR} \cdot \mathrm{ME}$ & $5-3$ & HS 5 & $5-6$ \\
\hline $\mathrm{PWR} \cdot \mathrm{BE}$ & $5-4$ & & \\
\hline LSPB & $5-5$ & & \\
\hline
\end{tabular}

\subsection{COST CHANGES}

The Phase VIII Update costs reflect the technical changes discussed in Section 4, including the commodity/equipment changes summarized in Tables 4-1 and 4-2. These changes were made to meet the primary objective of the Phase VIII Update, which was to refine the EEDB technical/cost data models to reflect current experience. The following paragraphs focus on the Phase VII to Phase VIII cost changes for the PWR, LSPB and HS5.

As was discussed in Section 4.3.1, "Review of Impact of Changing Regulations, Codes and Standards," none of the regulatory changes that have occurred between January 1, 1984 and January 1, 1986 required significant changes to be made to the quantities of commodities and equipment in the technical data models and, consequently, in the related cost data models. The changing regulations did have an impact on the indirect costs in the form of increases in craft, engineering and field supervision manhours. These cost increases were reflected in the overall increased manhours in the PWR Update. Insufficient data is available, however, to identify what portion of these increases are caused by the changed regulations.

A major emphasis in the Phase VIII Update was the detailed technical reviews and resulting technical changes described in Sections 4.3 .2 and 4.4. Specific cost changes to the Phase VII (1984) PWR $\cdot M E$, PWR $\cdot B E$, LSPB and HS5 were based on these changes. The PWR $\cdot M E, P W R \cdot B E$ and HS 5 cost changes were generated by the technical refinements resulting from the overall technical reviews of the PWR and HS5. The LSPB cost changes were based on the LSPB extension of detail and EEDB conformance activities. Additional cost changes were made to the $\mathrm{PWR} \cdot \mathrm{ME}, \mathrm{PWR} \cdot \mathrm{BE}, \mathrm{LSPB}$ and $\mathrm{HS} 5$ to reflect the effects of inflation that has occurred between the Phase VII and Phase VIII Updates, as discussed in Section 5.1 . 


\subsubsection{Presentation of the PWR and HS5 Cost Changes}

Tables 5-7 through 5-9 present the base construction cost changes that have occurred in the PWR $\cdot \mathrm{ME}, \mathrm{PWR} \cdot \mathrm{BE}$ and HS5, between the Phase VII (1984) and Phase VIII (1986) Updates. No table was prepared for the LSPB because the Phase VII Update LSPB data at the three-digit code-of-accounts level of detail was of insufficient depth to support this type of analysis. The Phase VII Update costs in these tables were increased by a uniform factor of approximately 7.4 percent, in order to express the Phase VII Update dollars in terms of Phase VIII Update dollars. The tables present the base construction costs for the Phase VII and Phase VIII Updates in terms of 1986 constant dollars, the differences between the Phase VII and Phase VIII Update costs, and the cost differences stated as a percentage of the inflated Phase VII costs. In these tables, the direct costs were regrouped from the building/system (Account) orientation of Tables 5-3 through 5-6, to a commodity/equipment orientation for Structural, Mechanical and Electrical/Instrumentation and Control (I\&C) items. Table 5-10 specifically indicates what is included in each of these accounts.

The direct equipment/labor cost accounts may be related to the direct building/ system cost accounts as follows. The Structural direct costs are the installed costs for structural commodities for the buildings in Accounts 21, 22 (HS5 only), and 26 plus the installed costs for equipment foundations and underground encasements for all accounts (Accounts 21 through 26). The Mechanical direct costs are the installed costs for the building service equipment, piping and ducting in Account 21 and for a11 of the mechanical equipment and piping in Accounts 22 through 26. The Electrical/I\&C direct costs are the installed costs for the lighting and service power systems in Account 21 , the I\&C equipment in Accounts 21 through 26, all of Account 24 (including the diesel-generator units) except foundations and underground encasements, and the communication equipment in Account 25.

\subsubsection{PWR ME Cost Changes (Table 5-7)}

The base construction costs for the PWR ME increased between the Phase VII and Phase VIII Updates at a rate which was seven percent above the general inflation rate. This increase was primarily attributable to the general technical refinements discussed in Section 4.4. The relationship of this cost change to previous EEDB and actual power plant cost trends is discussed in Sections 3.3 .3 and 3.4

\subsubsection{PWR.ME Direct Costs}

The direct costs increased by one percent above general inflation. The largest dollar cost increase above general inflation was in the "Mechanical (w/o NSSS + T/G)" account. This account increased by four percent above the general rate of inflation. The increase was primarily caused by the mechanical technical changes described in Section 4.4 .1 and by a change in the small bore piping installation rate to reflect current field data. 
The "NSSS + T/G" account kept pace with the general rate of inflation. Both the nuclear steam supply system (NSSS) and the turbine-generator unft (T/G) costs were based on 1986 vendor estimates.

The "Total Mechanical" account was driven to increase by two percent above inflation by the increase in the "Mechanical (w/o NSSS $+T / G$ )" account described above.

The "Structural" account decreased by two percent below general inflation. This was primarily caused by the structural technical changes described in Section 4.4.1 and structural labor costs that increased slower than inflation. The latter effect was caused by the fact that the structural account was highly labor intensive and the average structural labor escalation for the period $1984 / 1985$ was lower than the general inflation rate. The decrease was partly offset by an increase in structural installation rates based on field data.

The "Electrical/I\&C" account increased by four percent above general inflation because of the electrical/I\&C technical changes described in section 4.4.1. Factory equipment plus site material (FE + SM) costs were about equal to installation labor costs in this account. While labor costs increased at a rate higher than general inflation, they were partly offset by $\mathrm{FE}+\mathrm{SM}$ costs which increased at a lower rate than general inflation.

\subsubsection{PWR $\cdot$ ME Indirect Costs}

The indirect costs increased by seven percent above general inflation. Construction Services showed an increase of two percent above general inflation. This increase was caused by an increase in construction service craft labor hours to reflect current field data.

The Engineering and Home Office Services account increase of 27 percent above general inflation was caused by an increase in engineering hours to reflect current field experience and by an increase in salaries and expenses that was somewhat higher than general inflation. The Field Supervision and Field Office Services account increase of seven percent above general inflation was attributable to the increase in craft manhours associated with the changes described in Section 4.4.1, an increase in the percentage of supervisors to craftsmen to reflect current experience, and an increase in salaries and expenses that was higher than general inflation.

\subsubsection{PWR $\cdot \mathrm{BE}$ Cost Changes (Table 5-8)}

The base construction costs for the PWR $B E$ increased between the Phase VII and Phase VIII Updates at a rate that was two percent above the general inflation rate. This increase was primarily attributable to inflation and the general technical refinements discussed in Section 4.4. The relationship of this cost change to previous EEDB and actual power plant cost trends is discussed in Sections 3.3 .3 and 3.4 . 


\subsubsection{PWR BE Direct Costs}

The direct costs increased by two percent above general inflation. The largest dollar cost increase above general inflation was in the "Mechanical ( $w / 0$ NSSS $+T / G) "$ account. This account increased by four percent above the general rate of inflation for the same reasons as given for the similar increase in the PWR PE. The "NSSS + T/G" account kept pace with inflation and the "Total Mechanical" account increased by two percent above inflation for the same reasons given for similar effects in the PWR ME.

The "Structural" account for the PWR・BE increased by two percent above general inflation. The increase was primarily caused by increases in structural installation rates that were generally higher than similar increases made to the PWR $\cdot \mathrm{ME}$. Greater percentage increases were made to the $\mathrm{PWR} \cdot \mathrm{BE}$ in order to adjust the PWR $M E$ to $P W R \cdot B E$ structural installation rate ratios to reflect current field data. The increase was partly offset by the structural changes described in Section 4.4.1 that were very similar for both the PWR ${ }^{M E}$ and PWR $\cdot B E$ and by structural labor costs that increased more slowly than inflation as discussed in Section 5.4.2.1. The latter effect, however, did not influence the results for the $\mathrm{PWR} \cdot \mathrm{BE}$ as much as for the $\mathrm{PWR} \cdot \mathrm{ME}$ because the $\mathrm{PWR} \cdot \mathrm{BE}$ had lower overall structural manhours.

The "Electrical/I\&C" account increased at a rate that was four percent above that of general inflation because of the electrical/I\&C technical changes described in Section 4.4.1.

\subsubsection{2 $\mathrm{PWR} \cdot \mathrm{BE}$ Indirect Costs}

The indirect costs increased by three percent above general inflation. Construction Services showed an increase of three percent above inflation for the same reason given for the PWR $M E$ increase. The PWR $\cdot B E$ increase on a percentage basis is higher than that of the PWR ME because the increase was measured against a smaller base.

The Engineering and Home Office Services account increase of two percent above inflation was caused by an increase in salaries and expenses that was higher than the general inflation rate. The Field Supervision and Field office Services account increase of six percent above inflation was caused by the increase in craft manhours associated with the changes described in Section 4.4.1 and an increase in salaries and expenses that was higher than general inflation.

\subsubsection{LSPB Cost Changes}

The base construction cost for the LSPB increased between the Phase VII and Phase VIII Updates at a rate that was five percent above general inflation. This increase was caused by the expansion of detall and EEDB conformance activities described in Section 4.3.2.3 and the technical changes described in Section 4.4.2. 
Insufficient detail was available from the Phase VII Update LSPB to perform the type of direct and indirect cost change analysis reported for the PWR $\cdot \mathrm{ME}$ and $\mathrm{PWR} \cdot \mathrm{BE}$ above and the HS5 below.

\subsubsection{HS5 Cost Changes (Table 5-9)}

The base construction cost for the HS5 has essentially kept pace with inflation between the Phase VII and Phase VIII Updates.

\subsubsection{HS5 Direct Costs}

The direct costs decreased by one percent below general inflation, where the majox changes were decreases in the "FGD System" and "structural" accounts.

"FGD Systems" decreased by three percent below general inflation because the components of this equipment intensive account escalated at a rate that was much lower than inflation. The "FSSS $+\mathrm{T} / G$ " account kept pace with inflation for the same reasons given for simflar effects in the PWR data models. The "Other Mechanical" account kept pace with inflation because increases caused by the technical changes described in Section 4.4 .3 were offset by equipment prices that escalated at a rate lower than inflation. Consequently, the "Mechanical" account essentially kept pace with inflation because the major components of the account did so.

The "Structural" account decreased by four percent below inflation for the same reasons given for similar effects for the PWR ME. The percentage decrease, however, was higher than for the PWR ME because the decrease was measured against a smaller base.

The "Electxical/I\&C" account increased by two percent above inflation because of the technical changes described in Section 4.4.3.

\subsubsection{HS5 Indirect Costs}

The indirect costs increased by two percent above inflation. Construction services showed an increase of two percent above inflation for the same reason given for the PWR ME increase.

The Engineering and Home Office Services account increase of two percent above inflation was caused by an increase in salaries and expenses that was higher than the general inflation rate. The Field Supervision and Field office Services account increase of one percent above inflation was caused by a small increase in craft manhours associated with the changes described in Section 4.4.3 and a small increase above inflation for salaries and expenses.

5.5 BASIS FOR COST COMPARISONS FOR THE PWR •ME, THE PWR B BE, THE LSPB AND THE HS 5

Base construction costs for the PWR $\cdot M E, P W R \cdot B E$, LSPB and HS 5 are compared in Tables 5-11 and 5-12 on both a dollars and a dollars per kilowatt basis. 
Factory equipment plus site materials costs are given in Table 5-11, while labor (craft, engineering and field supervision) costs are given in Table 5-12.

Tables 5-11 and 5-12 have the base construction costs grouped in a commodity/ equipment and labor orientation for structural, mechanical and electrical items. These tables form the basis for Figures $3.1,3.2$ and 3.3 and for the direct cost portions of Tables 5-7, 5-8 and 5-9. Following is a listing of what is included in each account:

\subsubsection{Factory Equipment plus Site Material Costs (Table 5-11)}

- NSSS or FSSS - the price for the nuclear or fossil steam supply system scope of supply plus the cost of site materials required for its installation. When installation manhours are included in a quotation, quantities and costs of the installation manhours are requested. The installation costs are deducted from the quotation and the result is entered in the factory equipment account. The installation manhours are multiplied by Middletown labox rates and the resulting costs are entered in the labor account (Table 5-12).

- Flue Gas Desulfurization (FGD) System (Coal-Fired Power Plants only) - the quoted estimating cost of the flue gas desulfurization system and the site materials required for its installation. Costs of associated structural commodties, bullding or equipment foundations, draft booster fans, piping, HVAC equipment, I\&C equipment and electrical equipment are included in the appropriate accounts described below.

- T/G Unit - the price for the turbine-generator unit plus the cost of site materials required for its installation.

- Mechanical - the quoted estimating cost or apparatus handbook price of the mechanical equipment listed plus the cost of site materials required for their installation. Installation costs included in system/equipment quotations are deducted and distributed in the labor accounts as described in the "NSSS or FSSS" section. "Other Equipment" includes all other mechanical equipment not listed, such as tanks, filters and HVAC equipment.

- Piping - the cost for all interconnecting piping systems and equipment, except for the NSSS primary piping (included with the NSSS). PIping costs include costs for valves, supports, specialties and the site materials required for theix installation. 
- Electrical/I\&C - the quoted estimating cost or apparatus handbook price for all electrical, instrumentation and control equipment, including interconnecting wiring and its supporting or enclosing raceways. Electrical/I\&C costs include the costs of building lighting, building service power, communications equipment and site materials required for installation.

- Structural Support - the cost of the structural support commodities; structural steel, reinforcing steel, structural concrete, embedded steel and formwork, including fabricated items. These items are mostly site material, but include some fabricated items of factory equipment.

- Structural (Other) - the cost of all other structural and architectural commodities and items, such as roofing, interior and exterior non-load bearing walls, doors, miscellaneous steel, protective coatings, plus structure equipment, such as elevators. These items are mostly site material, but include some fabricated or manufactured items of factory equipment.

- Construction Services - the rental or purchase cost of and expenses associated with construction equipment (e.g., earth moving machinery and construction cranes), temporary buildings, small tools and expendable supplies plus field office expenses. These are indirect costs.

\subsubsection{Labor Costs (Table 5-12)}

- Structural Craft Labox - the installation labor costs for the construction of structures, buildings and other civil works, including equipment foundations.

- Mechanical Craft Labox - the installation labor costs for installing or erecting equipment and piping for the reactor or boiler plant, mechanical plant, miscellaneous plant, building services and heat rejection systems and equipment. All craft labor which is not in the structural or electrical accounts is included here.

- Electrical/I\&C Craft Labor - the installation labor costs for the installation of instrumentation, control, and electrical systems and equipment, including emergency engine-driven generators, building lighting, service power and communication systems.

- Construction Services - the costs for labor to construct and maintain temporaxy buildings and facilities, to store, maintain and repair construction tools and equipment, to 
service field office equipment, and to provide security for the area during construction; plus the costs for surveying team support, medical/first aid support, expendable supplies, and construction training meetings. These are indirect costs.

- Salaries - costs for englneering and engineering support, plus costs for non-manual field supervision personnel and services, including quality assurance/quality control, construction management and test/start-up personnel (supporting craft labor for testing/start-up is included in the craft labor categories above and is a direct cost) and services. These are indirect costs.

- Expenses - expenses associated with the personnel and services listed under salaries. These are indirect costs.

- Insurance/Taxes - costs of payroll insurance and taxes plus builders a11 risk insurance. These are indirect costs.

\subsubsection{Use of Commodity/Equipment/Labor Accounting System}

These nine equipment/material and seven labor cost sub-categories identify all of the cost components of the technical data models while segregating those items which have caused major cost differences among nuclear power plant construction projects. Examination of the numerical data in Tables 5-11 and 5-12 can provide many useful insights into the cost relationships of the PWR $\cdot M E$, PWR B BE, LSPB and HS5. For example, examination of the piping for these four plants on a dollars per kilowatt $(\$ / \mathrm{kWe})$ basis reveals the following relationships:

$1986 \$ /$ kWe Ratios

\begin{tabular}{|c|c|c|c|c|}
\hline Piping & $\begin{array}{c}\mathrm{PWR} \cdot \mathrm{ME} \\
1144 \mathrm{MWe} \\
\end{array}$ & $\begin{array}{c}\mathrm{PWR} \cdot \mathrm{BE} \\
1144 \mathrm{MWe} \\
\end{array}$ & $\begin{array}{c}\text { LSPB } \\
1311 \text { MWe } \\
\end{array}$ & $\begin{array}{c}\text { HS5 } \\
488 \text { MWe } \\
\end{array}$ \\
\hline $\begin{array}{l}\text { FE + SM } \\
\text { Labor } \\
\text { Total Costs }\end{array}$ & $\begin{array}{l}1.35 \\
2.69 \\
2.03\end{array}$ & $\begin{array}{l}\text { Base } \\
\text { Base } \\
\text { Base }\end{array}$ & $\begin{array}{l}0.83 \\
0.93 \\
0.88\end{array}$ & $\begin{array}{l}1.17 \\
0.82 \\
0.99\end{array}$ \\
\hline
\end{tabular}

Comparing the PWR $\cdot M E, L S P B$ and HS5 to the PWR BE indicates that the total piping installed costs on a $\$ / k W e$ basis are nearly equal for the PWR BE and HS5, about twice as high for the $P W R \cdot M E$ as for the $P W R \cdot B E$, and about 12 percent lower for the LSPB than for the PWR BE.

$\underline{\mathrm{PWR} \cdot \mathrm{ME} \text { Versus } \mathrm{PWR} \cdot \mathrm{BE}}$

The EEDB Phase VI Update $(1983)^{6}$ indicates that median cost experience plants typically have significantly lower labor productivities and higher material 
quantities than similar best cost experience plants. The above analysis has affirmed and quantified that the expected cost relationships have not changed. Factory equipment (FE) plus site material (SM) costs for the PWR $\cdot M E$ are 35 percent higher than for the PWR $B E$, reflecting the lower quantity (pounds) of pipe in the PWR BE based on current field data. For the PWR $\cdot M E$ piping, labor costs are over 2-1/2 times as high as for the PWR BE emphasizing the combination of higher quantities and lower labor productivity. It should be noted, however, that the lower productivity is the major contributor to the higher labor dollars for the PWR $\cdot \mathrm{ME}$.

$\underline{\text { LSPB Versus } \mathrm{PWR} \cdot \mathrm{BE}}$

Although LSPB piping quantities (pounds) are somewhat higher than PWR BE quantities, the LSPB $\$ / k W e$ for FE + SM, Labor and Total Costs are lower than for the $P W R \cdot B E$. The lower $\$ / k W e$ for the LSPB reflect the fact that the factory equipment costs for the primary and intermediate piping are included in the NSSS cost rather than the piping cost. Although this is also true for the PWR primary piping, there is a much larger quantity of piping in the LSPB primary and intermediate systems than for the PWR primary system. Another contributing factor to the lower LSPB $\$ / \mathrm{kWe}$ is the fact that the LSPB has a capacity (kWe) that is approximately 15 percent higher than that of the PWR. In addition, the LSPB has unique design features which reduce the amount of safety related piping compared to the $\mathrm{PWR} \cdot \mathrm{BE}$ which results in both lower material and lower labor costs.

\section{HS 5 Versus PWR・BE}

The $\$ / k W e$ similarity in the HS 5 and PWR BE piping total costs is a result of offsetting cost factors. The lower capacity of the HS5 produces higher $\$ / \mathrm{kWe}$ costs than for the high capacity PWR BE. Additionally, the higher operating pressures and temperatures of the HS5 produce higher unit piping costs than for the PWR $\cdot B E$. Offsetting these costs, however, are higher cost and lower labor productivities for safety-related piping in the PWR BE. Although PWR BE labor productivities for non-safety related piping are similar to HS5 piping productivities, PWR $\cdot B E$ safety related piping productivities are lower than those for the HS5 piping. Consequently, the analysis has qualitatively affirmed the expected cost relationships. 


\section{ENERGY ECONOMIC DATA BASE}

PHASE VIII UPDATE

MAJOR EQUIPMENT ESTIMATING QUOTATIONS RECEIVED FOR THE PWR, LSPB AND HS5 AND MAJOR EQUIPMENT ESTIMATES BASED ON ESTIMATING QUOTATIONS RECEIVED DURING THE PHASE VII UPDATE

Equipment

Ash Handling System

Boiler (NSSS or FSSS)

Chimney

Circulating Water Piping (Concrete)

Circulating Water Pumps

Coal Handling System

Condensate Polishing System

Condensate Pumps

Condensate Storage Tank

Condenser

Cooling Towers (Natural Draft)

Deaerating Heater

Electrostatic Precipitators

Emergency Diesel-Generator Units

Emergency Gas Turbine Generator Units

Feedwater Heaters

Feedwater Pumps

Flue Gas Booster Fans

Lime Transfer System

Major Cranes

Make-up Demineralizer

Make-up Water Pretreatment System

Radwaste Volume Reduction and

Solidification System

Service Water Pumps

Soot Blowing Air Compressor

Traveling Water Screens

Turbine-Generator Unit
$\frac{\mathrm{PWR}}{\mathrm{Q} \underline{\mathrm{E}}}$

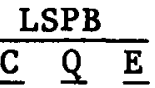

HS5

Q $\underline{E}$

$\mathrm{X}$

$\mathrm{X}$

$\mathrm{X}$

$\mathrm{X}$

$\mathrm{X}$

$\mathrm{X}$

$\mathrm{X}$

$\mathrm{X}$

$\begin{array}{lllll}x & x & & x \\ x & x & x & & x\end{array}$

$\mathrm{X}$

$\mathrm{X}$

$\mathrm{X}$

$\mathbf{X}$

$\mathrm{X}$

$\mathrm{X}$

$\mathrm{X}$

$\mathrm{X}$

$\mathrm{X}$

$\mathrm{X}$

$\mathrm{X}$

$\mathrm{X}$

$\mathrm{X}$

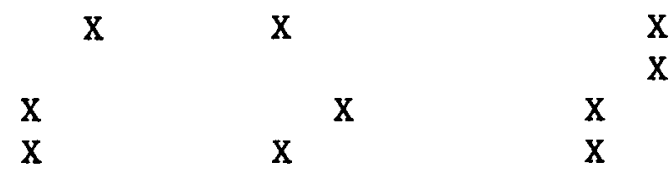

Q = Estimating Quotation Recelved

$\mathrm{E}=$ Estimate Based on Phase VII Estimating Quotations

$\mathrm{C}=$ Based on EPRI/CoMO Data 
TABLE 5-2

ENERGY ECONOMIC DATA BASE

PHASE VIII UPDATE

PREFERENTIAL AND DISCRETIONARY ITEMS EXCLUDED FROM EEDB

BASE CONSTRUCTION COSTS

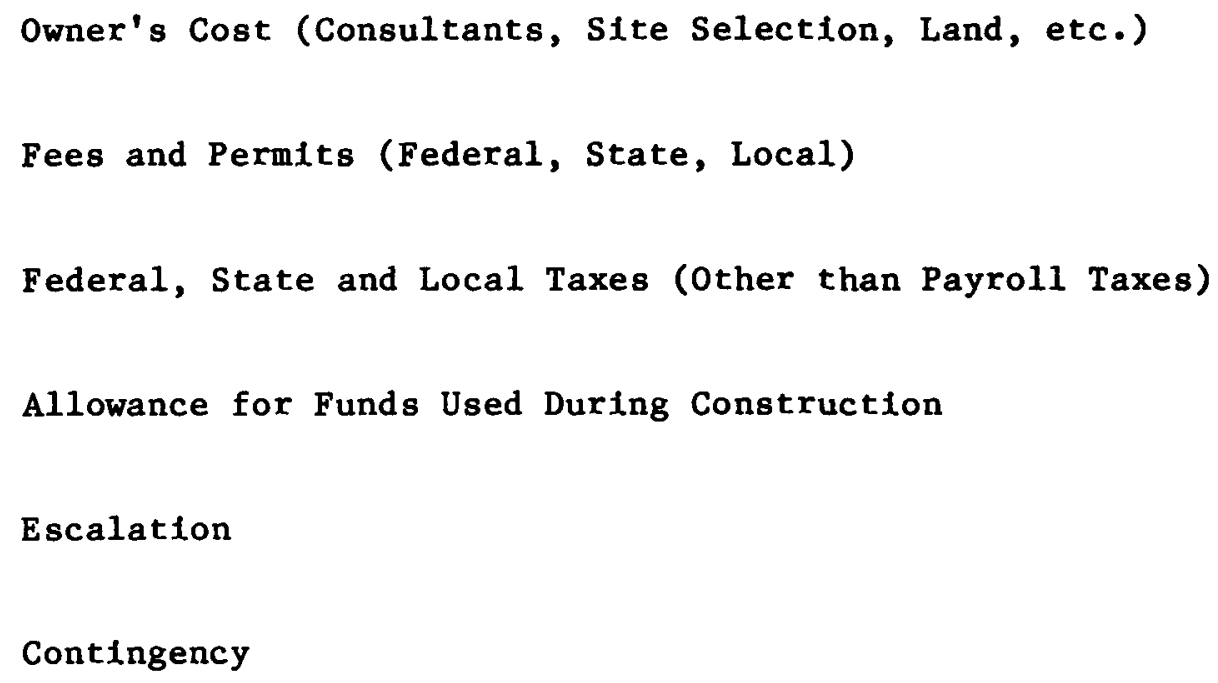


Effective Date $1 / 1 / 86$

TABLE 5-3

ENERGY ECONOMIC DATA BASE - PHASE VIII UPDATE

1144 MWe PRESSURIZED WATER REACTOR NPGS

MEDIAN EXPERIENCE (PWR $\cdot M E)$

BASE CONSTRUCTION COST ESTIMATE 


$\begin{array}{ll}\text { PLANT CODE } & \text { COST BASIS } \\ 148 & 01 / 86\end{array}$

\section{ACCT NO ACCOUNT DESCRIPTION}

************************************

21 STRUCTURES + IMPROVEMENTS

22 . REACTOR PLANT EQUIPMENT

23. TURBINE PLANT EQUIPMENT

24. ELECTRIC PLANT EQUIPMENT

25 . MISCELLANEOUS PLANT EQUIPT

26. MAIN COND HEAT REJECT SYS

TOTAL DIRECT COSTS

91 CONSTRUCTION SERVICES

92 . HOME OFFICE ENGRG. \&SERVICE

93. FIELD OFFICE ENGRG\&SERVICE

TOTAL INDIRECT COSTS

TOTAL BASE COST
UNITED ENGINEERS \& CONSTRUCTORS INC.

EEDB-VIII BASE COSTS (MEDIAN EXPERIENCE BASIS) 1144 MWE PRESSURIZED WATER REACTOR

\section{FACTORY} EQUIP. COSTS

$* * * * * * * * * * * * *$

$23,196,345$

$240,229,192$

$169,727,737$

$31.107,359$

$19,279,977$

$31,011,399$

$514,552,009$

$111,710.000$

$468,684,000$

$389,817,000$

$970.211,000$

$1.484,763,009$
SITE

LABOR HOURS

************

$9028599 \mathrm{MH}$

$4352219 \mathrm{MH}$

3154618 MH

2792072 MH

1730823 MH

$968055 \mathrm{MH}$

22026386 MH

$7523000 \mathrm{MH}$

$932000 \mathrm{MH}$

8455000 MH

$30481386 \mathrm{MH}$
SITE
LABOR COST *************

$187,850,927$

$102,814,880$

$73,734,538$

$64,568,507$

$40,939,697$

$19,335,234$

$489,243,783$

$167,067,000$

$20,719.000$

$187,786,000$

677.029 .783
SUMMARY PAGE

$09 / 16 / 86$

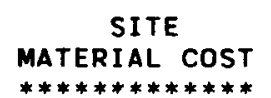

TOTAL

CosTS

*************

$89,525,350$

$20,654,982$

$11,383,997$

$18,251,812$

$6,281,689$

$3,410,625$

$149,508,455$

*********

$300,572,622$

$363,699,054$

$254,846,272$

$113,927.678$

$66,501,363$

$53,757,258$

$1,153,304,247$

$108,573,000$

$387,350,000$

$468,684,000$

$428,533,000$

$126,570,000$

1.284 .567 .000

$276.078,455$

$2.437 .871,247$ 


$\begin{array}{cc}\text { PLANT CODE } & \text { COST BASIS } \\ 148 & 01 / 86\end{array}$

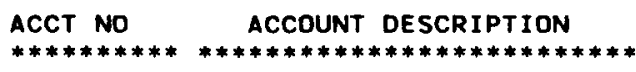

211. YARDWORK

212. REACTOR CONTAINMENT BLDG

213. TURBINE ROOM + HEATER BAY

214. SECURITY BUILOING

215. PRIM AUX BLDG + TUNNELS

216. WASTE PROCESS BUILDING

217. FUEL STORAGE BLDG

218A. CONTROL RM/D-G BUILDING

218B. ADMINISTRATION+SERVICE BLG

2180. FIRE PUMP HOUSE, INC FNDTNS

218E. EMERGENCY FEED PUMP BLDG

218F. MANWAY TUNNELS (RCA TUNLS)

218G. ELEC. TUNNELS

218H. NON-ESSEN. SWGR BLDG.

218U. MN STEAM + FW PIPE ENC.

218K. PIPE TUNNELS

218L. TECHNICAL SUPPORT CENTER

218P. CONTAIN EO HATCH MSLE SHLD

218S. WASTE WATER TREATMENT

218T. ULTIMATE HEAT SINK STRUCT

218V. CONTR RM EMG AIR INTK STR

21. STRUCTURES + IMPROVEMENTS

UNITED ENGINEERS \& CONSTRUCTORS INC.

EEDB-VIII BASE COSTS (MEDIAN EXPERIENCE BASIS)

1144 MWE PRESSURIZED WATER REACTOR

SUMMARY PAGE

$09 / 16 / 86$

FACTORY

EQUIP. COSTS

*************

285.069

$14,914,270$

562.599

77.239

2.992 .273

606.289

964,983

$1,637,755$

915.834

39,598

22,168

10,907

21.693

33.151

61.791

8,241

42.485

$23,196,345$
SITE

******** HOURS

1039008

SITE

LABOR COST

1039008 MH $19,514,914$

$3025169 \mathrm{MH}$

893357 MH

$52128 \mathrm{MH}$

779295 MH

$712439 \mathrm{MH}$

322642 MH

$924808 \mathrm{MH}$

271347 MH

15673 MH

124178 MH

$47558 \mathrm{MH}$

$2228 \mathrm{MH}$

$19462 \mathrm{MH}$

385721 MH

$18+10 \mathrm{MH}$

25241 MH

9571 MH

29307 MH

327198 MH

$4159 \mathrm{MH}$

$9028599 \mathrm{MH}$
$64,055,189$

$19,320,610$

$1,116,280$

$16,249,557$

$14,598,165$

$6,763,583$

$19,827,640$

5.856 .515

333.660

$2,545,324$

948,263

52.407

414,223

$8,058,310$

352,811

508,802

189,620

581,304

$6,483,324$

80,426

$187,850,927$

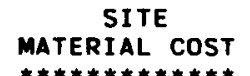

*************

$12,438,937$

20.972 .815

$17,314.877$

569.859

$6,579,110$

$6.685,912$

$5.068,904$

$8,245,719$

3.151 .926

158,410

999,125

312.189

20.404

284.775

$3,547,514$

120.557

263,405

57,779

298.141

$2.404,370$

30,622

$89,525,350$
TOTAL COSTS

$* * * * * * * * * * * * * *$

$32,238,920$

$99,942,274$

$37,198,086$

$1,763,378$

$25,820,940$

$21,890,366$

$12,797,470$

$29,711,114$

$9,924.275$

531.668

$3,566,617$

$1,260.452$

83,718

720,691

11.638 .975

473,368

833.998

247.399

887,686

8.930 .179

111,048

$300,572,622$ 


$\begin{array}{lc}\begin{array}{c}\text { PLANT CODE } \\ 148\end{array} & \text { COST BASIS } \\ & 01 / 86 \\ \text { ACCT NO } & \\ * * * * * * * * * * * * * * * * * * * * * * * * * * * * * * * * * * * *\end{array}$

220A. NUCLEAR STEAM SUPPLY(NSSS)

220B. NSSS OPTIONS

221. REACTOR EQUIPMENT

222. MAIN HEAT XFER XPORT SYS.

223. SAFEGUARDS SYSTEM

224. RADWASTE PROCESSING

225. FUEL HANDLING + STORAGE

226. OTHER REACTOR PLANT EQUIP

227. RX INSTRUMENTATION+CONTROL

228. REACTOR PLANT MISC ITEMS

22 REACTOR PLANT EQUIPMENT

231. TURBINE GENERATOR

233. CONDENSING SYSTEMS

234. FEED HEATING SYSTEM

235. OTHER TURBINE PLANT EQUIP.

236. INSTRUMENTATION + CONTROL

237. TURBINE PLANT MISC ITEMS

23 .

TURBINE PLANT EQUIPMENT
UNITED ENGINEERS \& CONSTRUCTORS INC

EEDB-VIII BASE COSTS (MEDIAN EXPERIENCE BASIS) 1144 MWE PRESSURIZED WATER REACTOR

$\begin{array}{cccc}\text { FACTORY } & \text { SITE } & \text { SITE } & \text { SITE } \\ \text { EQUIP. COSTS } & \text { LABOR HOURS } & \text { LABOR COST } & \text { MATERIAL COST } \\ * * * * * * * * * * * * & * * * * * * * * * * * & * * * * * * * * * * * * & * * * * * * * * * * * *\end{array}$

$176,000,000$

SUMMARY PAGE

$09 / 16 / 86$

************

$4.222 .940 \quad 5.960 .795$

$11,015,708$

$3,133,571$

$181940 \mathrm{MH}$

$15,365,085$

$1,546,926$

$20,045,582$

$7.776,809$

$636896 \mathrm{MH}$

$15,090,607$

1.676 .707

$24,544,123$

16.725 .094

$518762 \mathrm{MH}$

$12,277,686$

$2,247,112$

74632 MH

$1,763,273$

$1,261,420$

167,500

$30,264,200$

20.089 .817

$1672557 \mathrm{MH}$

39.613 .821

6.120 .589

659,460

$3.261,585$

$5,731,183$

$102,814,880$

$20,654,982$

$363,699,054$

\begin{abstract}
$120,820,989$
$16,634,731$

$12,902,988$
\end{abstract}

$465869 \mathrm{MH}$

$10,505,712$

$1,798,758$

$1,833,538$

$1,381,328$

$2,372,844$

456,425

$3,541,104$

$4,177,885$

65.824 .227

$22,834,561$

8.992 .768

$1,912,166$

$230900 \mathrm{MH}$

$5,344,226$

253500 MH

$5,826,481$

$73,734,538$

$11,383,997$

3154618 MH

$133,125,459$

$34,131,272$

$31,882,701$

$38,626,438$

$7,712,817$

$9,367,585$

$254,846,272$ 


$\begin{array}{cc}\text { PLANT CODE } & \text { COST BASIS } \\ 148 & 01 / 86\end{array}$

ACCT NO ACCOUNT DESCRIPTION $* * * * * * * * * * * * * * * * * * * * * * * * * * * * * * * * * * * *$

241. SWITCHGEAR

242. STATION SERVICE EQUIPMENT

243. SWITCHBOARDS

244. PROTECTIVE EQUIPMENT

245. ELECT.STRUC +WIRING CONTNR

246. POWER \& CONTROL WIRING

24. ELECTRIC PLANT EQUIPMENT

251. TRANSPORTATION \& LIFT EQPT

252. AIR, WATER+STEAM SERVICE SY

253. COMMUNICATIONS EQUIPMENT

254. FURNISHINGS + FIXTURES

255. WASTEWATER TREATMENT EQUIP

25. MISCELLANEOUS PLANT EQUiPT

261. STRUCTURES

262. MECHANICAL EQUIPMENT

26. MAIN COND HEAT REJECT SYS

TOTAL DIRECT COSTS

UNITED ENGINEERS \& CONSTRUCTORS INC.

EEDB-VIII BASE COSTS (MEDIAN EXPERIENCE BASIS) 1144 MWE PRESSURIZED WATER REACTOR

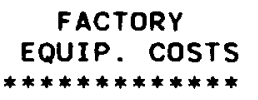

$11.090,208$

16.787 .134

1.546 .682

$1,683,335$

31.107 .359

$4,970,000$

$9,409.756$

$1.948,800$

$2,192,851$

758.570

$19,279,977$

177.249

$30,834,150$

31.011 .399

$514,552,009$

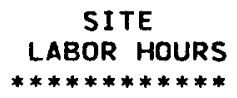

LABOR HOURS
$* * * * * * * * * * * *$

$25180 \mathrm{MH}$

$74182 \mathrm{MH}$

$16340 \mathrm{MH}$

$132050 \mathrm{MH}$

$1663710 \mathrm{MH}$

880610 MH

2792072 MH

$48600 \mathrm{MH}$

$1382113 \mathrm{MH}$

$192200 \mathrm{MH}$

$25910 \mathrm{MH}$

$82000 \mathrm{MH}$

$1730823 \mathrm{MH}$

$136614 \mathrm{MH}$

$831441 \mathrm{MH}$

$968055 \mathrm{MH}$

22026386 MH

\section{SITE}

LABOR COST

581,680

$1,659,532$

377,929

$3,079,537$

$38,333,122$

$20,536,707$

$64,568,507$

$1,148,298$

$32,737,715$

$4,482,296$

576,189

$1,995,199$

$40,939,697$

$2,783.012$

$16,552,222$

$19,335,234$

$489,243,783$
SUMMARY PAGE 4

$09 / 16 / 86$

\section{SITE}

MATERIAL COST

**************

TOTAL

Costs

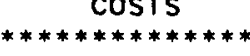

$11,757,518$

$18,768,111$

321,445

$2,051.237$

1.751 .225

$4,830,762$

$6,788.697$

$45,121,819$

$9,178,189$

$31,398,231$

$18,251,812$

$113,927,678$

114.830

5.261 .236

640,754

65,349

199.520

6.281 .689

$66,501,363$

1.487 .882

$4,448,143$

$1,922.743$

$49,309,115$

$3,410,625$

$53,757,258$

$149,508,455$

$1,153,304,247$ 


$\begin{array}{cc}\text { PLANT CODE } & \text { COST BASIS } \\ 148 & 01 / 86\end{array}$

\section{ACCT NO ACCOUNT DESCRIPTION \\ ************************************}

911. TEMPORARY CONSTRUCTION FAC

912. CONSTRUCTION TOOLS \& EQUIP

913. PAYROLL INSURANCE \& TAXES

914. PERMITS, INS. \& LOCAL TAXES

915. TRANSPORTATION

91 CONSTRUCTION SERVICES

921. HOME OFFICE SERVICES

922. HOME OFFICE Q/A

923. HOME OFFICE CONSTRCTN MGMT

92 - HOME OFFICE ENGRG.\&SERVICE

931. FIELD OFFICE EXPENSES

932. FIELD JOB SUPERVISION

933. FIELD OA/OC

934. PLANT STARTUP \& TEST

93. FIELD OFFICE ENGRG\&SERVICE

TOTAL INDIRECT COSTS

TOTAL BASE COST
UNITED ENGINEERS \& CONSTRUCTORS INC.

EEDB-VIII BASE COSTS (MEDIAN EXPERIENCE BASIS)

1144 MWE PRESSURIZED WATER REACTOR

SUMMARY PAGE

$09 / 16 / 86$
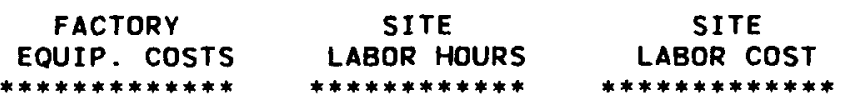

SITE
MATERIAL COST
$* * * * * * * * * * * *$

TOTAL

$* * * * * * * * * * * * *$

$\begin{array}{rr}7095000 \mathrm{MH} & 157,567,000 \\ 428000 \mathrm{MH} & 9,500,000\end{array}$

$33,578,000$

$72,557,000$

$111,710,000$

$2,438,000$ costs

$* * * * * * * * * * * * * *$

$191,145,000$

$82,057,000$

$111.710,000$

$2,438,000$

$111,710,000$

$7523000 \mathrm{MH}$

$167,067,000$

$108,573,000$

$387,350,000$

447.077 .000

$15,045,000$

$6,562.000$

$468,684,000$

$349,862,000$

$61000 \mathrm{MH}$

$1,358,000$

$17.997,000$

$447,077,000$

15.045 .000

$6,562,000$

$468,684,000$

$22.882,000$

$519000 \mathrm{MH}$

$11,533,000$

$352000 \mathrm{MH}$

$7,828,000$

$17,073,000$

$389,817,000$

$932000 \mathrm{MH}$

$20,719,000$

$17,997,000$

$970,211,000$

$8455000 \mathrm{MH}$

$187,786,000$

$126,570,000$

$1,284,567,000$

$1,484,763,009$

$30481386 \mathrm{MH}$

$677,029,783$

$276,078,455$

$2.437,871,247$
$428,533,000$

$361,395,000$

$30.710,000$

$17.073,000$ 
Effective Date $1 / 1 / 86$

TABLE 5-4

ENERGY ECONOMIC DATA BASE - PHASE VIII UPDATE

1144 MWe PRESSURIZED WATER REACTOR NPGS

BEST EXPERIENCE (PWR・BE)

BASE CONSTRUCTION COST ESTIMATE 


\section{PLANT CODE COST BASIS \\ 184$$
01 / 86
$$

\section{ACCT NO \\ ACCOUNT DESCRIPTION}

$* * * * * * * * * * * * * * * * * * * * * * * * * * * * * * * * * * * *$

21 . STRUCTURES + IMPROVEMENTS

22 . REACTOR PLANT EQUIPMENT

23 . TURBINE PLANT EQUIPMENT

24. ELECTRIC PLANT EQUIPMENT

25. MISCELLANEOUS PLANT EQUIPT

26 - MAIN COND HEAT REJECT SYS

TOTAL OIRECT COSTS

91 . CONSTRUCTION SERVICES

92 ENGINEERING \& H/O SERVICE

93 . FIELD SUPER. \& F/O SERVICE

TOTAL INDIRECT COSTS

TOTAL BASE COST

UNITED ENGINEERS \& CONSTRUCTORS INC.

EEDB-VIII BASE COSTS (BEST EXPERIENCE BASIS)

1144 MWE PRESSURIZED WATER REACTOR

SUMMARY PAGE

1

FACTORY EQUIP. COSTS *************

$22,368,321$

234.714 .431

$164,925,704$

31.043 .442

$17.999,000$

$30,494,602$

$501,545,500$

$60,227,000$

$204,632.000$

87.004 .000

$351,863,000$

$853,408,500$

\section{SITE}

LABOR HOURS

***********

5352863 MH

2026207 MH

1749871 MH

1447855 MH

895745 MH

$715791 \mathrm{MH}$

$12188332 \mathrm{MH}$

$4011000 \mathrm{MH}$

$424000 \mathrm{MH}$

$4435000 \mathrm{MH}$

$16623332 \mathrm{MH}$

\section{SITE}

LABOR COST

$110,715,738$

47.725 .800

$40,689,103$

33.410 .932

$21,162,093$

$13,895,141$

267.598 .807

$88,095,000$

9.318 .000

97.413 .000

$365,011,807$
$09 / 16 / 86$

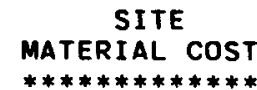

$64,316,744$

$15,607,693$

$8,191,814$

$13,854,516$

$5.024,698$

$1,922,465$

$108,917,930$

$62,844,000$

$211,166,000$

$204,632,000$

$107,578,000$

$74.100,000$

$523,376,000$

$183,017,930$ 


\begin{tabular}{|c|c|}
\hline $\begin{array}{l}\text { PLANT CODE } \\
184\end{array}$ & $\begin{array}{l}\text { COST BASIS } \\
01 / 86\end{array}$ \\
\hline $\begin{array}{l}\text { ACCT ND } \\
* * * * * * * *\end{array}$ & 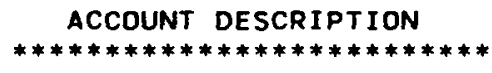 \\
\hline 211. & YARDWORK \\
\hline 212. & REACTOR CONTAINMENT BLDG \\
\hline 213. & TURBINE ROOM + HEATER BAY \\
\hline 214. & SECURITY BUILDING \\
\hline 215. & PRIM AUX BLDG + TUNNELS \\
\hline 216. & WASTE PROCESS BUILDING \\
\hline 217. & FUEL STORAGE BLDG \\
\hline $218 A$. & CONTROL RM/D-G BUILDING \\
\hline $218 B$. & ADMINISTRATION+SERVICE BLG \\
\hline 2180. & FIRE PUMP HOUSE, INC FNDTNS \\
\hline $218 E$. & EMERGENCY FEED PUMP BLDG \\
\hline $218 \mathrm{~F}$. & MANWAY TUNNELS (RCA TUNLS) \\
\hline 218G. & ELEC. TUNNELS \\
\hline $218 \mathrm{H}$. & NON-ESSEN. SWGR BLDG. \\
\hline $218 \mathrm{~J}$. & MN STEAM + FW PIPE ENC. \\
\hline $218 K$. & PIPE TUNNELS \\
\hline $218 L$. & TECHNICAL SUPPORT CENTER \\
\hline $218 P$. & CONTAIN EQ HATCH MSLE SHLD \\
\hline 2185. & WASTE WATER TREATMENT \\
\hline $218 T$. & ULTIMATE HEAT SINK STRUCT \\
\hline $218 V$. & CONTR RM EMG AIR INTK STR \\
\hline & ZUCTURES + IMPROVEMENTS \\
\hline
\end{tabular}

UNITED ENGINEERS \& CONSTRUCTORS INC.

EEDB-VIII BASE COSTS (BEST EXPERIENCE BASIS) 1144 MWE PRESSURIZED WATER REACTOR

\begin{tabular}{|c|c|c|}
\hline $\begin{array}{c}\text { FACTORY } \\
\text { EQUIP. COSTS } \\
* * * * * * * * * * * *\end{array}$ & $\begin{array}{c}\text { SITE } \\
\text { LABOR HOURS } \\
* * * * * * * * * * * *\end{array}$ & $\begin{array}{c}\text { SITE } \\
\text { LABOR COST } \\
* * * * * * * * * * * *\end{array}$ \\
\hline 243,826 & $734203 \mathrm{MH}$ & $13,617,699$ \\
\hline $14,560,308$ & $1667865 \mathrm{MH}$ & $35,665,296$ \\
\hline 553.291 & $503+48 \mathrm{MH}$ & 10.851 .127 \\
\hline 51.458 & $35768 \mathrm{MH}$ & 765,684 \\
\hline $2.904,343$ & $459941 \mathrm{MH}$ & 9.517 .441 \\
\hline 599,302 & $426404 \mathrm{MH}$ & $8,690,922$ \\
\hline 963,778 & $204071 \mathrm{MH}$ & $4,265,484$ \\
\hline 1.410 .991 & $501737 \mathrm{MH}$ & 10.509 .342 \\
\hline 853,742 & $148484 \mathrm{MH}$ & $3,173,039$ \\
\hline 39,321 & $10908 \mathrm{MH}$ & 230,147 \\
\hline \multirow[t]{2}{*}{22.036} & $81488 \mathrm{MH}$ & 1.664 .026 \\
\hline & $26155 \mathrm{MH}$ & 533,373 \\
\hline 9.532 & $1728 \mathrm{MH}$ & 40.623 \\
\hline 21.561 & $13186 \mathrm{MH}$ & 282,629 \\
\hline \multirow[t]{2}{*}{32,787} & 246412 МH & $5,128,257$ \\
\hline & $13323 \mathrm{MH}$ & 266,432 \\
\hline \multirow[t]{2}{*}{51,458} & $19592 \mathrm{MH}$ & 394.239 \\
\hline & $8386 \mathrm{MH}$ & 165,927 \\
\hline 8,233 & $23444 \mathrm{MH}$ & 468,450 \\
\hline \multirow[t]{2}{*}{42,354} & $223152 \mathrm{MH}$ & $4,418,193$ \\
\hline & 3468 МH & 67.408 \\
\hline $22,368,321$ & $5352863 \mathrm{MH}$ & $110,715,738$ \\
\hline
\end{tabular}

SUMMARY PAGE 2

$09 / 16 / 86$

MATERIAL COST

TOTAL Costs

$* * * * * * * * * * * * *$

$10,284,638$

$24,146,163$

$64,937,052$

$22,685,150$

$1,222,897$

$17,027,256$

$13,881.741$

$9,551,020$

17.209 .442

$6,404,444$

414,977

$2,455,488$

772,710

65,863

528,336

$7,702,204$

379,982

651,933

218, 269

746,374

$6,303,857$

95,645

$197,400,803$ 


$\begin{array}{cc}\text { PLANT CODE } & \text { COST BASIS } \\ 184 & 01 / 86\end{array}$

ACCT NO ACCOUNT DESCRIPTION
$* * * * * * * * * * * * * * * * * * * * * * * * * * * * * * * * * *$

220A. NUCLEAR STEAM SUPPLY(NSSS)

220B. NSSS OPTIONS

221. REACTOR EQUIPMENT

222. MAIN HEAT XFER XPORT SYS.

223. SAFEGUARDS SYSTEM

224. RADWASTE PROCESSING

225. FUEL HANDLING + STORAGE

226. OTHER REACTOR PLANT EOUIP

227. RX INSTRUMENTATION+CONTROL

228. REACTOR PLANT MISC ITEMS

22. REACTOR PLANT EQUIPMENT

231. TURBINE GENERATOR

233. CONDENSING SYSTEMS

234. FEED HEATING SYSTEM

235. OTHER TURBINE PLANT EQUIP.

236. INSTRUMENTATION + CONTROL

237. TURBINE PLANT MISC ITEMS

23. TURBINE PLANT EQUIPMENT

UNITED ENGINEERS \& CONSTRUCTORS INC.

EEDB-VIII BASE COSTS (BEST EXPERIENCE BASIS)

1144 MWE PRESSURIZED WATER REACTOR

SUMMARY PAGE 3

FACTORY
EQUIP. COSTS
$* * * * * * * * * * * *$

$176,000,000$
SITE
LABOR HOURS $* * * * * * * * * * * *$

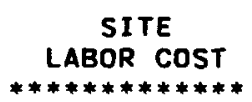

$* * * * * * * * * * * * *$
SITE

MATERIAL COST

$* * * * * * * * * * * * *$

159800 MH
263796 MH
227110 MH
165169 MH
35301 MH
678931 MH
319600 MH
176500 MH

$234,714,431$

120.662 .207

16.099, 766

$15,394,693$

$10,930,416$

$1,838,622$

$164,925,704$
$3,709,021$

6.238 .602

$5,379,366$

$3,900,318$

831.707

$16,070,984$

$7,404,412$

$4,191,390$

$47,725,800$

$7,422,594$

$7.881,758$

$6,924,943$

$9,501,793$

$4,411,475$

$4,546,540$

$40,689,103$
$5,909,403$

618.503

670,645

759,701

103,336

$3,817,652$

557.295

$3,171,158$

15.607 .693

$1,403,029$

1.133 .759

689,382

1.154 .803

379,118

$3,431,723$

$8,191,814$
$09 / 16 / 86$

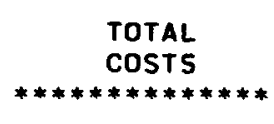

$176,000,000$

$10,425,222$

$9,734,262$

$12,869,327$

$20,613,263$

3. 126.961

$37,046,157$

$20.870,184$

$7,362,548$

298.047 .924

$129,487,830$

25.115 .283

$23,009,018$

21.587 .012

$6,629,215$

$7,978,263$

$213,806,621$ 


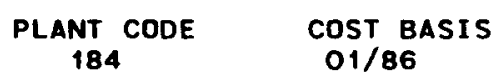

\section{ACCT NO ACCOUNT DESCRIPTION}

$* * * * * * * * * * * * * * * * * * * * * * * * * * * * * * * * * * * *$

241. SWITCHGEAR

242. STATION SERVICE EOUIPMENT

243. SWITCHBOARDS

244. PROTECTIVE EQUIPMENT

245. ELECT.STRUC +WIRING CONTNR

246. POWER \& CONTROL WIRING

24. ELECTRIC PLANT EQUIPMENT

251. TRANSPORTATION \& LIFT EQPT

252. AIR, WATER+STEAM SERVICE SY

253. COMMUNICATIONS EOUIPMENT

254. FURNISHINGS + FIXTURES

255. WASTE WATER TREATMENT EQ

25. MISCELLANEOUS PLANT EQUIPT

261. STRUCTURES

262. MECHANICAL EQUIPMENT

26. MAIN COND HEAT REJECT SYS

TOTAL DIRECT COSTS

UNITED ENGINEERS \& CONSTRUCTORS INC.

EEDB-VIII BASE COSTS (BEST EXPERIENCE BASIS) 1144 MWE PRESSURIZED WATER REACTOR

FACTORY

EQUIP. COSTS

$\underset{* * * * * * * * * * * *}{ }$

11.095 .979

$16,817,676$

$1,546,682$

$1.583,105$

31.043 .442

$4,970,000$

$8,189,860$

1.948 .800

$2,131,770$

758,570

$17,999,000$

173,725

$30,320,877$

$30,494,602$
SITE
LABOR COST

$* * * * * * * * * * * * *$

581,680

$1,539,381$

339,747

2.393 .899

17.287 .223

$11,269.002$

33.410 .932

824,600

$14,307,733$

$3,719,700$

485,182

$1,824,878$

$21,162.093$

1.986 .742

$11,908,399$

$13,895,141$

$715791 \mathrm{MH}$

$12188332 \mathrm{MH}$
SUMMARY PAGE 4

$09 / 16 / 86$

\begin{tabular}{rr}
$\begin{array}{r}\text { SITE } \\
* \text { TERIAL COST }\end{array}$ & \multicolumn{1}{c}{$\begin{array}{l}\text { TOTAL } \\
\text { COSTS }\end{array}$} \\
85,630 & $11,763,289$ \\
293,421 & $18,650.478$ \\
123,507 & $2,009,936$ \\
1.751 .225 & $4,145.124$ \\
$4.320,156$ & $21,607.379$ \\
$7,280,577$ & $20,132,684$ \\
$13,854,516$ & $78,308,890$
\end{tabular}

82,461

5.877 .061

$4,305,160$

580.120

$26,802,753$

$6,248,620$

$2,673,909$

$2,583,448$

$44,185,791$

$3,328,585$

$42,983,623$

$46,312,208$
$1,168,118$

1.922 .465

$108,917,930$ 


\begin{tabular}{|c|c|}
\hline $\begin{array}{l}\text { PLANT CODE } \\
184\end{array}$ & $\begin{array}{l}\text { COST BAStS } \\
01 / 86\end{array}$ \\
\hline $\begin{array}{l}\text { ACCT NO } \\
* * * * * * * * * *\end{array}$ & 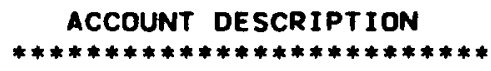 \\
\hline 911. & TEMPORARY CONSTRUCTION FAC \\
\hline 912. & CONSTRUCTION TOOLS E EQUIP \\
\hline 913. & PAYROLL INSURANCE TAXES \\
\hline 914. & PERMITS,INS. LOCAL TAXES \\
\hline 915. & TRANSPORTATION \\
\hline 91. & CONSTRUCTION SERVICES \\
\hline 921. & HOME OFFICE SERVICES \\
\hline 922. & HOME OFFICE Q/A \\
\hline 923. & HOME OFFICE CONSTRCTN MGMT \\
\hline 92 . & ENGINEERING \& H/O SERVICE \\
\hline 934 . & FIELD OFFICE EXPENSES \\
\hline 932. & FIELD JOB SUPERVISION \\
\hline 933. & FIELD OA/OC \\
\hline 934. & PLANT STARTUP \& TEST \\
\hline 93 & FIELD SUPER. \& F/O SERVICE \\
\hline
\end{tabular}

TOTAL INDIRECT COSTS

TOTAL BASE COST
UNITED ENGINEERS \& CONSTRUCTORS INC.

EEDB-VIII BASE COSTS (BEST EXPERIENCE BASIS) 1144 MWE PRESSURIZED WATER REACTOR

FACTORY
EQUIP. COSTS

**************

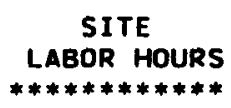
SITE
LABOR COST $* * * * * * * * * * * * *$

***********

$3666000 \mathrm{MH}$

$80,492,000$

$345000 \mathrm{MH}$

$7.603,000$

$60,227,000$

$4011000 \mathrm{MH}$

88.095 .000

$193,215,000$

6.936 .000

$4.481,000$

204.632 .000

70.272 .000
$5.873,000$
10.859 .000
$87.004,000$

$351,863,000$

$853,408,500$

424000 MH

$9,318,000$

4435000 MH

$97,413,000$

$16623332 \mathrm{MH}$

$365,011,807$
SUMMARY PAGE 5

$09 / 16 / 86$

TOTAL

MATERIAL COST

$* * * * * * * * * * * * *$

CosTs

$19,211,000$

$99,703,000$

$42,232,000$

49.835 .000

$60,227,000$

1.401 .000

1.401 .000

$62.844,000$

$211.166,000$

$193,215,000$

$6.936,000$

4.481 .000

204.632 .000

$\begin{array}{rrrr}26000 \mathrm{MH} & 593,000 & 11,256,000 & 11.849,000 \\ 300000 \mathrm{MH} & 6.584 .000 & 76.856 .000 \\ 98000 \mathrm{MH} & 2,141.000 & 8.014 .000 \\ & & 10.859 .000\end{array}$

$11,256,000$

$107,578,000$

$74,100,000$

$523,376,000$

$183,017,930$

$1,401,438,237$ 
Effective Date $1 / 1 / 86$

\section{TABLE 5-5}

ENERGY ECONOMIC DATA BASE - PHASE VIII UPDATE

1311 MWe LARGE SCALE PROTOTYPE BREEDER NPGS (LSPB)

BASE CONSTRUCTION COST ESTIMATE 


\section{PLANT CODE \\ 430 \\ COST BASIS \\ $01 / 86$}

ACCT NO

ACCOUNT DESCRIPTION *************************************

21. STRUCTURES + IMPROVEMENTS

22 - REACTOR PLANT EOUIPMENT

23. TURBINE PLANT EQUIPMENT

24. ELECTRIC PLANT EQUIPMENT

25. MISCELLANEOUS PLANT EQUIPT

26. MAIN COND HEAT REJECT SYS

TOTAL DIRECT COSTS

91 . CONSTRUCTION SERVICES

92 ENGINEERING \& H/O SERVICE

93. FIELD SUPER. \& F/O SERVICE

TOTAL INDIRECT COSTS

TOTAL BASE COST

UNITED ENGINEERS \& CONSTRUCTORS INC.

EEDB-VIII BASE COSTS (BEST EXPERIENCE BASIS)

1311 MWE LARGE SCALE PROTOTYPE BREEDER

FACTORY EQUIP. COSTS

$* * * * * * * * * * * *$

$20,458,157$

$643,970,807$

$196,016,400$

$35.023,581$

$39,180,534$

$29,864,469$

$964,513,948$

$78,304,000$

$278,851,000$

$112,048,000$

$469,203,000$

$1.433,716,948$

\section{SITE}

LABOR HOURS ************

7094475 MH

$3128383 \mathrm{MH}$

2009708 MH

$1742898 \mathrm{MH}$

$1157830 \mathrm{MH}$

$680215 \mathrm{MH}$

15813509 MH

$4936000 \mathrm{MH}$

$664000 \mathrm{MH}$

5600000 MH

21413509 MH
SITE

LABOR COST $* * * * * * * * * * * * *$

$149,772,660$

$73,663,053$

$46,627,500$

$39,984,438$

$27,282,467$

13. 174.672

$350,504,790$

109.384 .000

$14.681,000$

$124,065,000$

$474,569,790$
SUMMARY PAGE

$09 / 30 / 86$

SITE
MATERIAL Cost $* * * * * * * * * * * * *$

$117,297,899$

21,249,655

$9,436,476$

$19,847,621$

$5,821,292$

$2,552,721$

$176,205,664$

$92,253,000$

$13,132,000$

$105,385,000$

TOTAL

***************

$287,528,716$

$738,883.515$

$252,080,376$

$94,855,640$

$72,284,293$

$45,591,862$

$1,491,224,402$

$279.941,000$

$278,851,000$

$139.861,000$

$698,653,000$

$281,590,664$

$2,189,877,402$ 


$\begin{array}{cc}\text { PLANT CODE } & \text { COST BASIS } \\ 430 & 01 / 86\end{array}$

\section{ACCT NO ACCOUNT DESCRIPTION}

$* * * * * * * * * * * * * * * * * * * * * * * * * * * * * * * * * * * *$

211. YARDWORK

212. REACTOR CONTAINMENT BLDG

213. TURBINE ROOM + HEATER BAY

214. GATE HOUSE

215. REACTOR SERVICE BUILOING

216. NUCLEAR ISLAND MAINT. BLDG

217. FUEL STORAGE BLDG

218A. CONTROL BUILDING

218B. PLANT SERVICE (ADMIN) BLDG

218C. GAS TURBINE BUILDING

2180. FIRE PUMP HOUSE, INC FNDTNS

218E. STEAM GENERATOR BUILDING

218H. ELECTRICAL EOUIPMENT BLDG

2181. AUXILIARY BUILDINGS

218K. PIPE TUNNELS

218N. MAINTENANCE SHOP + WAREHSE

218R. AUXILIARY BOILER BUILDING

218S. WASTE WATER TREATMENT

218T. INTERM. SODIUM STOR. VLTS

21. STRUCTURES + IMPROVEMENTS

UNITED ENGINEERS \& CONSTRUCTORS INC.

EEDB-VIII BASE COSTS (BEST EXPERIENCE BASIS)

1311 MWE LARGE SCALE PROTOTYPE BREEDER

FACTORY

EQUIP. COSTS

$* * * * * * * * * * * * *$

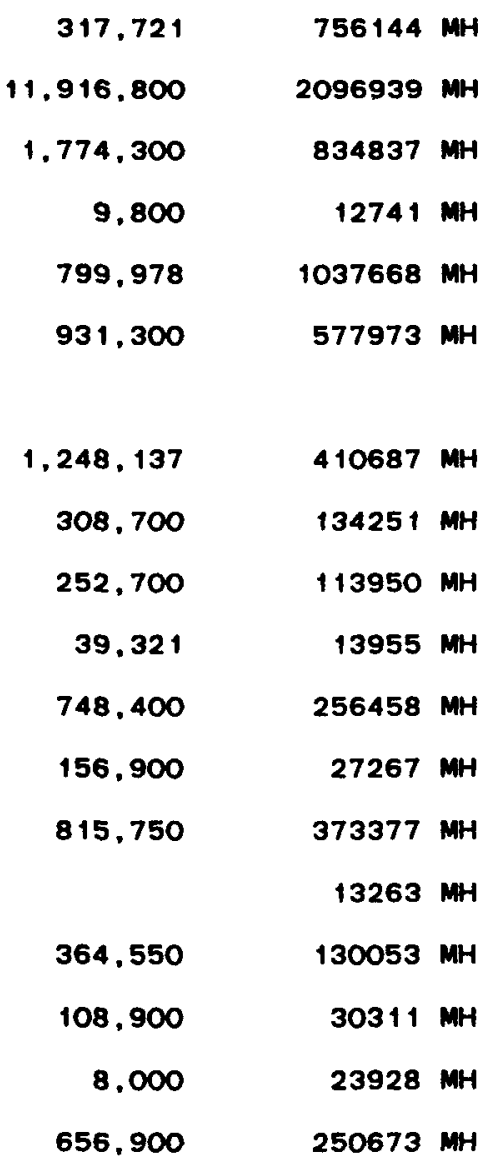

$20,458,157$
SITE

LABOR HOURS

$* * * * * * * * * *$

LABOR COST

$* * * * * * * * * * * * *$

$13,989,539$

45.891 .441

$18,464,244$

278,264

$21,920,266$

$12,064,862$

$8,559.719$

$2,853,765$

$2,347,873$

289.928

$5,481,005$

608,331

$7,621,913$

265,033

$2.789,728$

653,505

478,206

$5,215,038$

7094475 MH

$149,772,660$
SUMMARY PAGE

2

$09 / 30 / 86$

SITE

MATERIAL COST

$* * * * * * * * * * * * *$

TOTAL

**************4)

11.459 .856

$25,767,116$

$39,445,584$

$97,253,825$

$41,607,765$

522,123

$37,452,801$

$14,732,557$

$6,538,088$

$19,534,250$

$5,430,632$

$15,238,488$

$5,279,652$

$3,986,416$

505,390

$9,285,354$

$1,303,382$

$12,880,711$

378,038

$5,539,922$

$1,269,054$

760.099

$8,964,330$

$3,092,392$

$117,297,899$

$287,528,716$ 


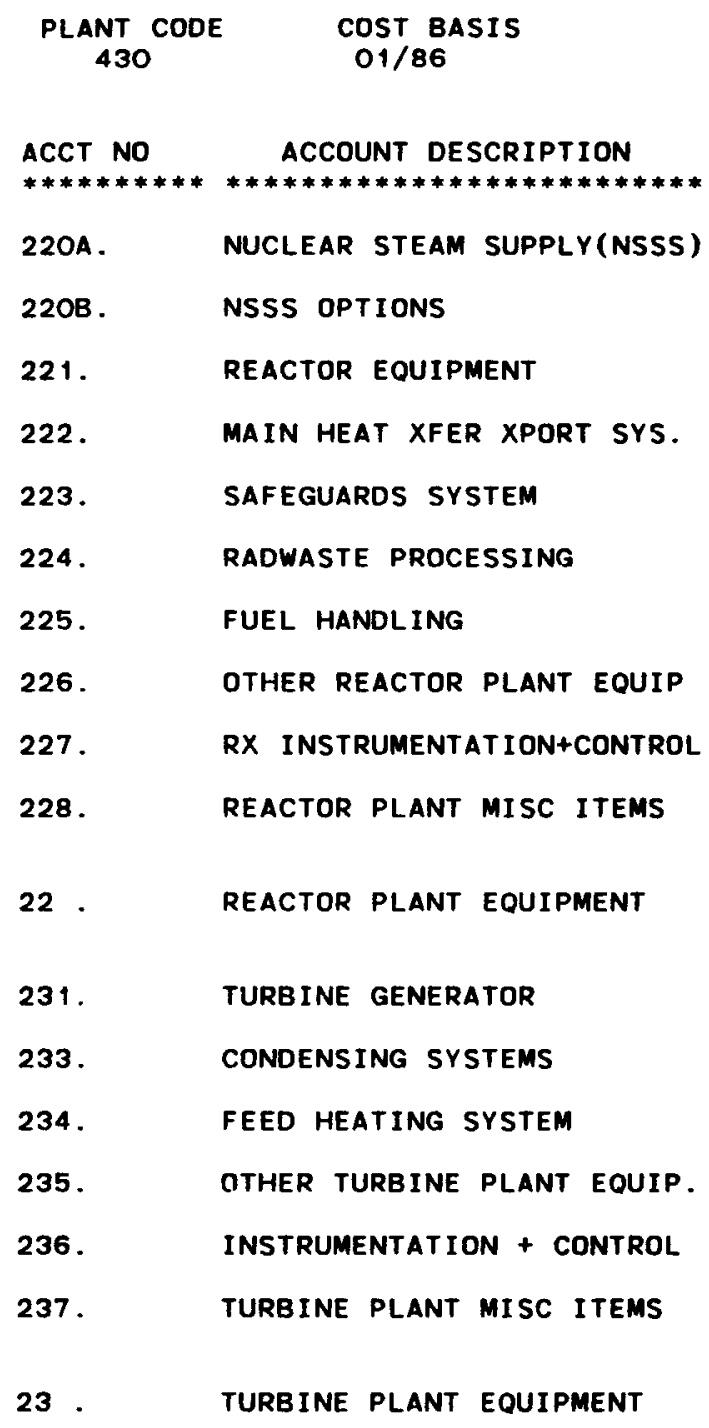

UNITED ENGINEERS \& CONSTRUCTORS INC.

EEDB-VIII BASE COSTS (BEST EXPERIENCE BASIS)

1311 MWE LARGE SCALE PROTOTYPE BREEDER

SUMMARY PAGE

$\begin{array}{ccc}\text { FACTORY } & \text { SITE } & \text { SITE } \\ \text { EQUIP. COSTS } & \text { LABOR HOURS } & \text { LABOR COST } \\ * * * * * * * * * * * * & * * * * * * * * * * * & * * * * * * * * * * * *\end{array}$

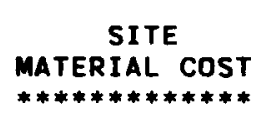

$618,877,462$

LABOR COST

$* * * * * * * *$

\begin{tabular}{|c|c|c|c|c|c|}
\hline 100,000 & 155200 & MH & $3,600,175$ & $5,535,966$ & $9,236,141$ \\
\hline $3,270,711$ & 1173615 & MH & $27,736,251$ & $2,764,136$ & $33,771,098$ \\
\hline 32,837 & 58019 & MH & $1,365,910$ & 136,593 & $1,535,340$ \\
\hline $5,135,063$ & 110901 & $\mathbf{M H}$ & $2,596,975$ & 427,338 & $8,159,376$ \\
\hline 89.456 & 141159 & MH & 3.290 .081 & 282,718 & $3,662,255$ \\
\hline $6,084,193$ & 566359 & MH & $13,288,884$ & $5,653,766$ & $25,026,843$ \\
\hline \multirow[t]{2}{*}{$10,381.085$} & 581130 & MH & $13,497,922$ & $1,076,991$ & $24,955,998$ \\
\hline & 342000 & MH & $8,286,855$ & $5,372,147$ & $13,659,002$ \\
\hline $643,970,807$ & 3128383 & MH & $73,663,053$ & $21,249,655$ & $738,883,515$ \\
\hline $134,317,906$ & 390643 & MH & 8.657 .577 & $1,875,640$ & $144,851,123$ \\
\hline $26,363,431$ & 484068 & MH & $11,385,892$ & $1,539,671$ & 39.288 .994 \\
\hline 22.294 .570 & 370560 & MH & 8.783 .513 & 872,465 & 31.950 .548 \\
\hline $11,653,918$ & 367637 & MH & $8,701,741$ & $1,025,873$ & $21,381,532$ \\
\hline \multirow[t]{2}{*}{1.386 .575} & 181600 & MH & $4,203,169$ & 376,925 & $5,966,669$ \\
\hline & 215200 & MH & $4,895,608$ & $3,745,902$ & $8,641,510$ \\
\hline 196.016 .400 & 2009708 & MH & $46,627,500$ & $9,436,476$ & $252,080,376$ \\
\hline
\end{tabular}




\begin{tabular}{|c|c|}
\hline $\begin{array}{l}\text { PLANT CODE } \\
430\end{array}$ & $\begin{array}{l}\text { COST BASIS } \\
01 / 86\end{array}$ \\
\hline $\begin{array}{l}\text { ACCT NO } \\
* * * * * * * * * *\end{array}$ & $\begin{array}{l}\text { ACCOUNT DESCRIPTION } \\
* * * * * * * * * * * * * * * * * * * * * * * * * * * *\end{array}$ \\
\hline 241. & SWI TCHGEAR \\
\hline 242. & STATION SERVICE EQUIPMENT \\
\hline 243. & SWITCHBOARDS \\
\hline 244. & PROTECTIVE EQUIPMENT \\
\hline 245. & ELECT.STRUC +WIRING CONTNR \\
\hline 246. & POWER \& CONTROL WIRING \\
\hline 24 & ELECTRIC PLANT EQUIPMENT \\
\hline 251. & TRANSPORTATION \& LIFT EOPT \\
\hline 252. & AIR, WATER+STEAM SERVICE SY \\
\hline 253. & COMMUNICATIONS EOUIPMENT \\
\hline 254. & FURNISHINGS + FIXTURES \\
\hline 255. & WASTE WATER TREATMENT EQ \\
\hline 25 & MISCELLANEOUS PLANT EQUIPT \\
\hline 261. & STRUCTURES \\
\hline 262. & MECHANICAL EQUIPMENT \\
\hline & MAIN COND HEAT REJECT SYS \\
\hline
\end{tabular}

TOTAL DIRECT COSTS
UNITED ENGINEERS \& CONSTRUCTORS INC.

EEDB-VIII BASE COSTS (BEST EXPERIENCE BASIS)

1311 MWE LARGE SCALE PROTOTYPE BREEDER

SUMMARY PAGE

4

\begin{tabular}{|c|c|c|c|c|c|}
\hline $\begin{array}{c}\text { FACTORY } \\
\text { EQUIP. COSTS } \\
* * * * * * * * * * * *\end{array}$ & $\begin{array}{c}\text { SITE } \\
\text { LABOR HOL } \\
* * * * * * * * * *\end{array}$ & & $\begin{array}{c}\text { SITE } \\
\text { LABOR COST } \\
* * * * * * * * * * * * *\end{array}$ & $\begin{array}{c}\text { SITE } \\
\text { MATERIAL COST } \\
* * * * * * * * * * * * *\end{array}$ & $\begin{array}{c}\text { TOTAL } \\
\text { COSTS } \\
* * * * * * * * * * * * * *\end{array}$ \\
\hline 12.745 .059 & 36330 & MH & 839.253 & 111.387 & 13.695 .699 \\
\hline $19,003,336$ & 94628 & MH & $2,135,795$ & 451,857 & $21,590,988$ \\
\hline \multirow[t]{3}{*}{$1,356.860$} & 11520 & MH & 266.563 & 135,114 & 1.758 .537 \\
\hline & 110400 & MH & $2,574,638$ & $1,829,262$ & $4,403,900$ \\
\hline & 943215 & $M H$ & $21,416,148$ & $6.413,621$ & $27,829,769$ \\
\hline $1.918,326$ & 546805 & MH & $12,752,041$ & $10,906,380$ & $25,576,747$ \\
\hline $35,023,581$ & 1742898 & MH & $39,984,438$ & $19,847,621$ & $94,855,640$ \\
\hline $5,489,800$ & 50575 & MH & $1,194,967$ & 119,496 & $6,804,263$ \\
\hline $27.999,565$ & 815455 & MH & $19,230,047$ & $4,813,571$ & $52,043,183$ \\
\hline $2,518,427$ & 194500 & $M H$ & $4,535,935$ & 661,744 & $7,716,106$ \\
\hline $2,414,172$ & 22300 & MH & 496,640 & 43,993 & $2,954,805$ \\
\hline 758.570 & 75000 & MH & $1.824,878$ & 182.488 & 2.765 .936 \\
\hline $39,180,534$ & 1157830 & MH & $27,282,467$ & $5,821,292$ & $72,284,293$ \\
\hline 158,192 & 97905 & MH & 1.987 .296 & $1,161,957$ & $3,307,445$ \\
\hline 29.706 .277 & 582310 & MH & $11,187,376$ & 1.390 .764 & $42,284,417$ \\
\hline $29,864,469$ & 680215 & MH & $13,174,672$ & $2,552,721$ & $45,591,862$ \\
\hline $964,513,948$ & 15813509 & MH & $350,504,790$ & $176,205,664$ & $, 491,224,402$ \\
\hline
\end{tabular}




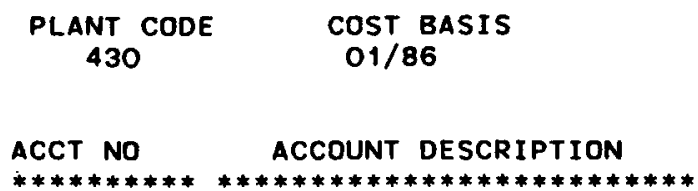

911. TEMPORARY CONSTRUCTION FAC

912. CONSTRUCTION TOOLS \& EQUIP

913. PAYROLL INSURANCE \& TAXES

914. PERMITS, INS. \& LOCAL TAXES

915. TRANSPORTATION

91 CONSTRUCTION SERVICES

921. HOME OFFICE SERVICES

922. HOME OFFICE $Q / A$

923. HOME OFFICE CONSTRCTN MGMT

92. ENGINEERING \& H/O SERVICE

931. FIELD OFFICE EXPENSES

932. FIELD JOB SUPERVISION

933. FIELD QA/OC

934. PLANT STARTUP \& TEST

93 . FIELD SUPER. \& F/O SERVICE

TOTAL INDIRECT COSTS

TOTAL BASE COST
UNITED ENGINEERS \& CONSTRUCTORS INC.

EEDB-VIII BASE COSTS (BEST EXPERIENCE BASIS)

1311 MWE LARGE SCALE PROTOTYPE BREEDER

SUMMARY PAGE 5

\begin{tabular}{|c|c|c|c|}
\hline $\begin{array}{c}\text { FACTORY } \\
\text { EOUIP. COSTS } \\
* * * * * * * * * * * * *\end{array}$ & $\begin{array}{c}\text { SITE } \\
\text { LABOR HOURS } \\
* * * * * * * * * * * *\end{array}$ & $\begin{array}{c}\text { SITE } \\
\text { LABOR COST } \\
* * * * * * * * * * * * *\end{array}$ & $\begin{array}{c}\text { SITE } \\
\text { MATERIAL COST } \\
* * * * * * * * * * * * *\end{array}$ \\
\hline & $4361000 \mathrm{MH}$ & $96,649,000$ & $23,871,000$ \\
\hline & $575000 \mathrm{MH}$ & $12,735, \infty 00$ & $66,192,000$ \\
\hline
\end{tabular}

$09 / 30 / 86$

$78,304,000$

$2,190,000$

TOTAL

COSTS

$* * * * * * * * * * * * * *$

$120,520,000$

$78,927,000$

$78,304,000$

2, 190,000

$78,304,000$

$4936000 \mathrm{MH}$

$109,384,000$

$92,253,000$

$279,941,000$

$263,291,000$

$9,453,000$

6.107 .000

$278,851,000$

$32000 \mathrm{MH}$

693,000

$13,132,000$

$11,184,000$

$90,500,000$

$505000 \mathrm{MH}$

$2,804,000$

$13,984,000$

$112,048,000$

$664000 \mathrm{MH}$

$14,681,000$

$13,132,000$

$263,291,000$

$9,453,000$

$6,107,000$

$278,851,000$

$13,825,000$

101.684 .000

$10,368,000$

$13,984,000$

$139,861,000$

$469,203,000$

5600000 MH

$124,065,000$

$105,385,000$

$698,653,000$

$1,433,716,948$
$474,569,790$
$281,590,664$ 
Effective Date $1 / 1 / 86$

TABLE 5-6

\author{
ENERGY ECONOMIC DATA BASE - PHASE VIII UPDATE \\ 488 MWe HIGH SULFUR COAL FPGS (HS5) \\ BASE CONSTRUCTION COST ESTIMATE
}




PLANT CODE
669

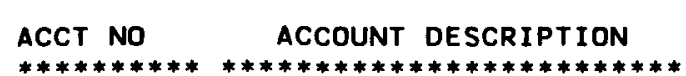

21. STRUCTURES + IMPROVEMENTS

22 . BOILER PLANT EQUIPMENT

23. TURBINE PLANT EQUIPMENT

24 . ELECTRIC PLANT EQUIPMENT

25. MISCELLANEOUS PLANT EQUTPT

26. MAIN COND HEAT REJECT SYS

TOTAL DIRECT COSTS

91 CONSTRUCTION SERVICES

92. ENGINEERING \& H/O SERVICE

93 . FIELD SUPER. \& F/O SERVICE

TOTAL INDIRECT COSTS

TOTAL BASE COST
UNITED ENGINEERS \& CONSTRUCTORS INC. ENERGY ECONOMIC DATA BASE (EEDB)

488 MWE HIGH SULFUR COAL

\begin{tabular}{|c|c|c|c|c|c|}
\hline $\begin{array}{c}\text { FACTORY } \\
\text { EQUIP. COSTS } \\
* * * * * * * * * * * * *\end{array}$ & $\begin{array}{c}\text { SITE } \\
\text { LABOR HOL } \\
* * * * * * * * * *\end{array}$ & & $\begin{array}{c}\text { SITE } \\
\text { LABOR COST } \\
* * * * * * * * * * * * *\end{array}$ & $\begin{array}{c}\text { SITE } \\
\text { MATERIAL COST } \\
* * * * * * * * * * * * *\end{array}$ & $\begin{array}{c}\text { TOTAL } \\
\text { COSTS } \\
* * * * * * * * * * * * * *\end{array}$ \\
\hline $2,533,768$ & 1328288 & MH & $27,123,384$ & $38,340,558$ & $67,997,710$ \\
\hline $148,942,415$ & 2314999 & MH & $53,962,151$ & 15.736 .650 & $218,641,216$ \\
\hline $69,187,230$ & 788439 & MH & $18,209,181$ & $4,589,384$ & $91,985,795$ \\
\hline 13.391 .645 & 671940 & MH & 15.514 .141 & $9,456,566$ & $38,362,352$ \\
\hline $8,900,633$ & 484339 & MH & $11,510,907$ & $1,979.757$ & $22,391,297$ \\
\hline $12,412,957$ & 340055 & MH & $6,592,558$ & $1,957,391$ & $20,962,906$ \\
\hline $255,368,648$ & 5928060 & MH & 132.912 .322 & $72,060,306$ & $460,341,276$ \\
\hline 24.973 .000 & 824000 & MH & $18,433,000$ & 20.539 .000 & $63,945,000$ \\
\hline $28,314,000$ & & & & & $28,314,000$ \\
\hline $18,854,000$ & & & & $1,418,000$ & $20,272,000$ \\
\hline $72,141,000$ & 824000 & MH & $18,433,000$ & 21.957 .000 & $112.531,000$ \\
\hline $327,509,648$ & 6752060 & MH & $151,345,322$ & 94.017 .306 & $572,872,276$ \\
\hline
\end{tabular}




$\begin{array}{cc}\text { PLANT CODE } & \text { COST BASIS } \\ 669 & 01 / 86\end{array}$

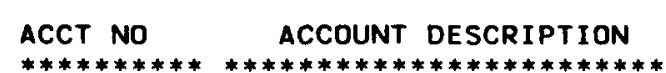

211. YARDWORK

212. STEAM GENERATOR BUILDING

213. TURBINE, HEATER, CONTROL BLD

218B. ADMINISTRATION+SERVICE BLD

219I. ELECTRICAL SWITCHGR BLDGS

218L. STACK/RECLAIM TRANSFR TWR

218M. COAL CAR THAW SHED

218N. ROTARY CAR DUMP BLDG+TUNNL

2180. COAL BREAKER HOUSE

218P. COAL CRUSHER HOUSE

2180. BOILER HOUSE TRANSFR TOWER

218R. DEAD STRG TRANSFER TUNNEL

2185. WASTE WATER TREATMENT BLDG

218T. LOCOMOTIVE REPAIR GARAGE

218U. MATERIAL HANDL+SERVICE BLD

218W. MISC COAL HANDLING STRUCT

219. STACK STRUCTURE

21. STRUCTURES + IMPROVEMENTS
UNITED ENGINEERS \& CONSTRUCTORS INC.

ENERGY ECONOMIC DATA BASE (EEDB)

488 MWE HIGH SULFUR COAL

FACTORY

EOUIP. COSTS

SITE
LABOR HOURS

SITE

LABOR COST

$* * * * * * * * * * * * *$

SITE
MATERIAL COST
$* * * * * * * * * * * * *$

$09 / 16 / 86$

TOTAL

\begin{tabular}{|c|c|c|c|c|c|}
\hline 54,184 & 277645 & MH & $5,007,388$ & $4,789,552$ & $9,851,124$ \\
\hline 778,447 & 352777 & MH & $7,658,184$ & $14,765,000$ & $23,201,631$ \\
\hline 484.010 & 248308 & MH & $5,400,381$ & $8.818,712$ & $14,703,103$ \\
\hline 475,509 & 64066 & MH & $1,408,915$ & 1.426 .643 & $3,311,067$ \\
\hline 12.186 & 2057 & MH & 44,149 & 18,140 & 74,475 \\
\hline \multirow[t]{2}{*}{5.775} & 8883 & MH & 193.727 & 252,627 & 452,129 \\
\hline & 6100 & MH & 122.184 & 51.286 & 173.470 \\
\hline 5,890 & 22146 & $\mathbf{M H}$ & 442,304 & 280,556 & 728,750 \\
\hline 103,152 & 20661 & MH & 452.660 & 638,291 & $1,194,103$ \\
\hline 124,452 & 13267 & MH & 293,328 & 334,233 & 752.013 \\
\hline \multirow[t]{2}{*}{2,438} & 5221 & MH & 117.440 & 169,625 & 289,503 \\
\hline & 17725 & MH & 355,231 & 197.491 & 552,722 \\
\hline 14.422 & 27708 & $M H$ & 558,765 & 456,771 & $1,029,958$ \\
\hline 19.557 & 8911 & MH & 195.598 & 229.950 & 445.105 \\
\hline 23,216 & 9441 & MH & 199,666 & 222,775 & 445,657 \\
\hline 284.790 & 130862 & MH & $2,443,823$ & $1,949,465$ & $4,678,078$ \\
\hline 145.740 & 112510 & $M H$ & $2,229,64 t$ & 3.739 .441 & 6.114 .822 \\
\hline 33,768 & 1328288 & MH & $27,123,384$ & $38,340,558$ & 67.997 .710 \\
\hline
\end{tabular}




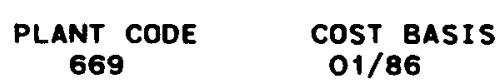

\section{ACCT NO ACCOUNT DESCRIPTION $* * * * * * * * * * * * * * * * * * * * * * * * * * * * * * * * * * * *$}

220A. FOSSIL STEAM SUPPLY SYSTEM

221. STEAM GENERATING SYSTEM

222. DRAFT SYSTEM

223. ASH + DUST HANDLING SYSTEM

224. FUEL HANDLING SYSTEMS

225. FLUE GAS DESULFUR STRUCT

226. DESULFURIZATION EQUIPMENT

227. INSTRUMENTATION + CONTROL

228. BOILER PLANT MISC ITEMS

22 . BOILER PLANT EQUIPMENT

231. TURBINE GENERATOR

233. CONDENSING SYSTEMS

234. FEED HEATING SYSTEM

235. OTHER TURBINE PLANT EQUIP.

236. INSTRUMENTATION + CONTROL

237. TURBINE PLANT MISC ITEMS

23 . TURBINE PLANT EQUIPMENT

UNITED ENGINEERS \& CONSTRUCTORS INC.

ENERGY ECONOMIC DATA BASE (EEDB)

488 MWE HIGH SULFUR COAL

SUMMARY PAGE

FACTORY

EQUIP - COSTS

**************

56, 300,000

1.075. 106

$7,537,672$

$5,429.199$

$19,184,685$

$2,389,654$

$52,174,897$

$4,585,036$

266,166

$148,942,415$

$46,275,110$

5.942 .319

$8,065,496$

8.762 .628

141,677

69.187 .230
SITE

LABOR HOURS

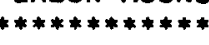

550000 MaH

$16896 \mathrm{MH}$

166256 Мн

$112193 \mathrm{MH}$

$216967 \mathrm{MH}$

254262 MH

853242 MH

77758 MH

$67425 \mathrm{MH}$

$2314999 \mathrm{MH}$

$200354 \mathrm{MH}$

76644 MH

$134163 \mathrm{MH}$

$311142 \mathrm{MH}$

$692 \mathrm{MH}$

$65444 \mathrm{MH}$

788439 MH SITE
LABOR COST
$* * * * * * * * * * * *$

$12,815,000$

401.593

$3,868,521$

2.642 .967

$5.122,871$

$5,454,236$

$20,446,843$

$1,799,735$

$1,410,385$

$53,962,151$

$4,463,828$

1.791 .391

$3,177,977$

$7,362,800$

16,003

$1,397,182$

$18,209,18$

$09 / 16 / 86$

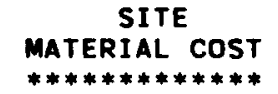

$1,281.500$

45,157

$2,113,096$

301.761

911,328

$6,747,666$

2.833.093

127.783

$1,375,266$

$15,736,650$

$1,154,013$

411.472

319,849

944,116

800

$1,759,134$

$4,589,384$
TOTAL

Costs

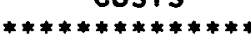

$70,396,500$

1.521 .856

$13,519,289$

8.373.927

$25,218,884$

$14,591,556$

$75,454,833$

$6,512,554$

$3,051,817$

$218,641,216$

$51.892,951$

8. 145.182

$11,563,322$

$17.069,544$

158,480

3.156 .316

$91,985,795$ 


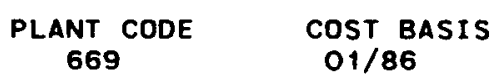

\section{ACCT NO}

ACCOUNT DESCRIPTION

************************************

241. SWITCHGEAR

242. STATION SERVICE EOUIPMENT

243. SWITCHBOARDS

244. PROTECTIVE EQUIPMENT

245. ELECT.STRUC +WIRING CONTNR

246. POWER \& CONTROL WIRING

24 . ELECTRIC PLANT EOUIPMENT

251. TRANSPORTATION \& LIFT EQPT

252. AIR,WATER+STEAM SERVICE SY

253. COMMUNICATIONS EQUIPMENT

254. FURNISHINGS + FIXTURES

255. WASTE WATER TREATMENT EQPT

25. MISCELLANEOUS PLANT EQUIPT

261. STRUCTURES

262. MECHANICAL EOUIPMENT

26. MAIN COND HEAT REJECT SYS

TOTAL DIRECT COSTS
UNITED ENGINEERS \& CONSTRUCTORS INC.

ENERGY ECONOMIC DATA BASE (EEDB)

488 MWE HIGH SULFUR COAL

SUMMARY PAGE

\begin{tabular}{|c|c|c|c|c|c|}
\hline $\begin{array}{c}\text { FACTORY } \\
\text { EQUIP. COSTS } \\
* * * * * * * * * * * * *\end{array}$ & $\begin{array}{c}\text { SITE } \\
\text { LABOR HOL } \\
* * * * * * * * * *\end{array}$ & & $\begin{array}{c}\text { SITE } \\
\text { LABOR COST } \\
* * * * * * * * * * * * *\end{array}$ & $\begin{array}{l}\text { SITE } \\
\text { MATERIAL COST } \\
* * * * * * * * * * * * *\end{array}$ & $\begin{array}{c}\text { TOTAL } \\
\text { COSTS } \\
* * * * * * * * * * * * * *\end{array}$ \\
\hline $6,884,258$ & 16210 & MH & 374,466 & 37.447 & $7,296,171$ \\
\hline $5,073,960$ & 26003 & MH & 579,414 & 138,979 & $5,792,353$ \\
\hline \multirow[t]{3}{*}{836,902} & 8830 & MH & 204,223 & 102.642 & 1.143 .767 \\
\hline & 81030 & MH & $1,891,614$ & $1,313,310$ & 3.204 .924 \\
\hline & 361570 & MH & $8,306,358$ & $2,892,765$ & $11,199,123$ \\
\hline 596,525 & 178297 & MH & $4,158,066$ & $4,971,423$ & 9.726 .014 \\
\hline $13.391,645$ & 671940 & MH & $15,514,141$ & $9,456,566$ & $38,362,352$ \\
\hline $2,074,625$ & 7610 & MH & 178.215 & 17.822 & $2,270,662$ \\
\hline 4.607 .634 & 278125 & MH & $6.582,730$ & $1,295,929$ & $12,486,293$ \\
\hline 191.260 & 48500 & MH & $1,131,069$ & 273,178 & $1,595,507$ \\
\hline 869,832 & 10130 & MH & 224,372 & 32,818 & $1,127,022$ \\
\hline 1.157 .282 & 139974 & MH & $3,394,521$ & 360,010 & 4.911 .813 \\
\hline $8,900,633$ & 484339 & MH & $11,510,907$ & $1,979,757$ & $22,391,297$ \\
\hline 96.884 & 74341 & MH & $1.525,160$ & $1,299,781$ & $2,921,825$ \\
\hline $12,316,073$ & 265714 & MH & $5.067,398$ & 657,610 & $18,041,081$ \\
\hline 12.412 .957 & 340055 & MH & $6.592,558$ & $1,957.391$ & $20,962,906$ \\
\hline 255.368 .648 & 5928060 & MH & $132,912,322$ & $72,060,306$ & 460.341 .276 \\
\hline
\end{tabular}




\section{PLANT CODE COST BASIS \\ 669 \\ $01 / 86$}

\section{ACCT NO}

ACCOUNT DESCRIPTION

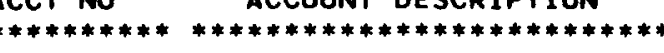

911. TEMPORARY CONSTRUCTION FAC

912. CONSTRUCTION TOOLS \& EOUIP

913. PAYROLL INSURANCE \& TAXES

914. PERMITS, INS. \& LOCAL TAXES

915. TRANSPORTATION

91 CONSTRUCTION SERVICES

921. HOME OFFICE SERVICES

922. HOME OFFICE Q/A

923. HOME OFFICE CONSTRCTN MGMT

92. ENGINEERING \& H/O SERVICE

931. FIELD OFFICE EXPENSES

932. FIELD JOB SUPERVISION

933. FIELD QA/QC

934. PLANT STARTUP \& TEST

93 . FIELD SUPER. \& F/O SERVICE

TOTAL INDIRECT COSTS

TOTAL BASE COST
UNITED ENGINEERS \& CONSTRUCTORS INC.

ENERGY ECONOMIC DATA BASE (EEDB)

488 MWE HIGH SULFUR COAL

SUMMARY PAGE 5

$09 / 16 / 86$

FACTORY EOUIP. COSTS $* * * * * * * * * * * * *$

\section{SITE}

LABOR HOURS

LABOR HOURS

LABOR COST

$\begin{array}{ll}703000 \mathrm{MH} & 15,730,000 \\ 121000 \mathrm{MH} & 2,703,000\end{array}$

$2,703,000$

$24,973,000$

824000 MH

$18,433,000$

$26.023,000$

$2,291,000$

$28,314,000$

$17,619,000$

483,000

752,000

$18,854,000$

72.141 .000

824000 MH

$18,433,000$

$327,509,648$
$151,345,322$
$20,539,000$

$63,945,000$

$21,957,000$

$112,531,000$

TOTAL

Costs

MATERIAL COST

6.509 .000

$22.239,000$

$16,160,000$

$24,973,000$

573.000

$26,023,000$

$2,291,000$

$28,314,000$

1.418 .000

$17,619,000$

483,000

752,000

$20,272,000$

$1.418,000$

$572,872,276$ 
TABLE $5-7$

ENERGY ECONOMIC DATA BASE

PHASE VIII UPDATE

PHASE VII TO PHASE VIII UPDATE BASE CONSTRUCTION COST INCREASES FOR THE PRESSURIZED WATER REACTOR NUCLEAR POWER GENERATING STATION -

MEDIAN EXPERIENCE (PWR -ME)

Commodity/Equipment/Service

$\mathrm{NSSS}+\mathrm{T} / \mathrm{G}(\mathrm{c})$

Mechanical ( $w / 0$ NSSS $+\mathrm{T} / G$ )

Sub-Total Mechantcal

Structural

Electrical/I\&C

Total Direct Costs (TDC)

Construction Services

Eng - and Home Office Services

Field Sup, and Field office Services

Total Indirect Costs (TIC)

TOTAL BASE CONSTRUCTION COSTS (TDC + TIC) $\frac{\text { Installed Costs }\left(\$ 1986 \times 10^{6}\right)(a)}{\text { Phase VII Phase VIII Delta }}$ as a \% of $\underline{(1984)} \quad$ (1986) Delta Phase VII(b)

313

312

(1)

$(\star)$

378

394

16

4

691

706

15

2

293

286

( 7)

(2)

155

161

6

4

1139

1153

14

1

379

387

8

2

369

469

100

27

401

429

28

7

1149

1285

136

12

2288

2438

150

7

(a) Data in Constant $\$ 1986$

(b) Inflation from $1 / 1 / 84$ to $1 / 1 / 86$ is taken as approximately 7.4 Percent

(c) Nucleax Steam Supply System Plus Turbine-Generator Untt

* Less than One Percent 
TABLE 5-8

ENERGY ECONOMIC DATA BASE

PHASE VIII UPDATE

PHASE VII TO PHASE VIII UPDATE BASE CONSTRUCTION COST INCREASES FOR THE PRESSURIZED WATER REACTOR NUCLEAR POWER GENERATING STATION -

BEST EXPERIENCE (PWR・BE)

Commodity/Equipment/Service

NSSS $+\mathrm{T} / \mathrm{G}(\mathrm{c})$

Mechantcal (w/o NSSS $+\mathrm{T} / G)$

Sub-Total Mechanical

Structural

Electrical/I\&C

Total Direct Costs (TDC)

Construction Services

Eng - and Home Office Services

Fleld Sup. and Fleld office Services

Total Indirect Costs (TIC)

TOTAL BASE CONSTRUCTION COSTS $(T D C+T I C)$

\begin{tabular}{|c|c|c|c|}
\hline Installed & $\$ 1986$ & $0, c$ & De1ta \\
\hline $\begin{array}{l}\text { Phase VII } \\
(1984)\end{array}$ & $\begin{array}{c}\text { Phase VIII } \\
\text { (1986) }\end{array}$ & Delta & $\begin{array}{l}\text { as a \% of } \\
\text { Phase VII (b) }\end{array}$ \\
\hline 312 & 311 & ( 1$)$ & (*) \\
\hline
\end{tabular}

242

$\underline{252}$

10

4

554

563

9

2

191

195

4

2

115

120

5

4

860

878

18

2

205

211

6

3

201

205

4

2

101

107

6

6

507

$\underline{523}$

16

3

1367

1401

34

2

(a) Data in Constant $\$ 1986$

(b) Inflation from $1 / 1 / 84$ to $1 / 1 / 86$ is taken as approximately 7.4 Percent

(c) Nuclear Steam Supply System Plus Turbine-Generator Unit

* Less than One Pexcent 
TABLE 5-9

ENERGY ECONOMIC DATA BASE

PHASE VIII UPDATE

PHASE VII TO PHASE VIII UPDATE BASE CONSTRUCTION COST INCREASES FOR THE 488 MWe HIGH SULFUR COAL-FIRED POWER GENERATING STATION (HS5)

\begin{tabular}{|c|c|c|c|c|}
\hline Commodity/Equipment/Service & $\begin{array}{c}\text { Installed } \\
\text { Phase VII } \\
(1984) \\
\end{array}$ & $\begin{array}{c}\text { sts }(\$ 1986 \\
\text { Phase VIII } \\
(1986) \\
\end{array}$ & $\frac{\left.10^{6}\right)(a)}{\text { Delta }}$ & $\begin{array}{r}\text { Delta } \\
\text { as a } \% \text { of } \\
\text { Phase VII (b) } \\
\end{array}$ \\
\hline FGD System $(c)$ & 59 & 57 & (2) & (3) \\
\hline FSSS $+T / G(d)$ & 120 & 119 & (1) & $(*)$ \\
\hline Other Mechanical & 146 & 147 & 1 & * \\
\hline Sub-Total Mechanical & 325 & 323 & (2) & $(*)$ \\
\hline Structural & 89 & 85 & (4) & (4) \\
\hline Electrical/I\&C & 51 & 52 & 1 & 2 \\
\hline Total Direct Costs (TDC) & 465 & 460 & (5) & (1) \\
\hline Construction Services & 63 & 64 & 1 & 2 \\
\hline Eng. and Home Office Services & 28 & 29 & $\#$ & 2 \\
\hline $\begin{array}{l}\text { Field Sup. and Field office } \\
\text { Services }\end{array}$ & 20 & 20 & $\#$ & 1 \\
\hline Total Indirect Costs (TIC) & 111 & 113 & 2 & 2 \\
\hline $\begin{array}{l}\text { TOTAL BASE CONSTRUCTION COSTS } \\
\text { (TDC + TIC) }\end{array}$ & 576 & 573 & (3) & $(*)$ \\
\hline
\end{tabular}

(a) Data in Constant $\$ 1986$

(b) Inflation From $1 / 1 / 84$ to $1 / 1 / 86$ is taken as approximately 7.4 Percent

(c) Flue Gas Desulfurization System: Does Not Include Commodities for Structures and Equipment Foundations; Draft Booster Fans; Piping; or HVAC, $I \& C$, and Electrical Equipment

(d) Fossil Steam Supply System plus Turbine-Generator Unit

* Less Than One Percent \# Less Than One 


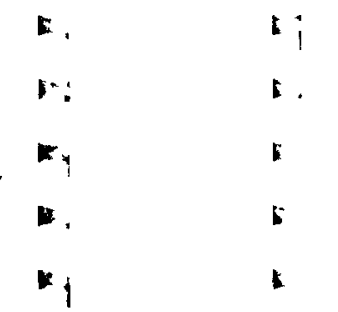

$\begin{array}{ll}1 & 1 \\ 1 & 1 \\ 5 & 1 \\ m & 1\end{array}$

1
1
1
1
+1

1
1
$m$
$m$

\section{$\underline{\text { DIRECT COSTS }}$}

FGD System (HS5 Only) - Flue gas desulfurization system plus site materials required for its installation (does not include commodities for structures and equipment foundations; draft booster fans; piping; or HVAC, I\&C, and electrical equipment). NSSS or FSSS + T/s - Nuclear or fossil steam supply system scope of supply
plus the turbine-generator unit plus site materials required for their tnstallation.

Mechanical (w/o NSSS + TG) - All mechanical components, equipment and interconnecting piping, including ductwork plus site material required for their installation.

Structura1 - All structural (e.g., concrete, reinforcing steel, formwork) and architectural (e.g., roofing, doors, miscellaneous steel) commodities plus structure equipment (e.g., elevators).

Electrical/IdC - All electrical, instrumentation and control equipment plus interconnecting wiring and associated raceways, including building lighting and service power, communications equipment and site materials required for their Installation.

\section{INDIRECT COSTS}

Construction Services - Temporary construction factilities, construction tools and equipment, payroll insurance and taxes, and builder's all-risk insurance.

Engineering and Home Office Services - Home office and field engineering services, home office quality assurance (QA) services and home office constructs and purchesing and expediting.

Field Supervision and Field Office Services - Field office expenses, field job supervision, field office quality assurance/quality control (QA/QC) services, and start-up and testing services. 
ENERGY ECONOMIC DATA BASE

PHASE VIII UPDATE

COMPARISON OF THE PWR $\cdot M E$, PWR $\cdot$ BE, LSPB, AND HS

DACTORY EQUIPMENT PLUS SITE MATERIAL COSTS

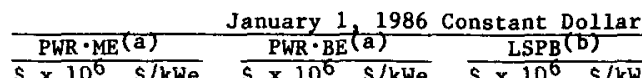

NSSS or FSSS :

FGD System:(d)

T/O Unit:

Mechanical

lectrica1/I\&C:

I

Structural Support

$$
\begin{aligned}
& \text { Concrete/Erbedded Steel } \\
& \text { Structural Steel } \\
& \text { Reinforcing Steel } \\
& \text { Formwork } \\
& \text { Sub-Total - Struc. Support }
\end{aligned}
$$

Structural (Other)

Construction Serv: (f) Ma for Equipment

Temp. Bldgs./Facilitie

Field Office Expense

Expendable Supplies/Safety

$$
\text { Small Tools }
$$

Sub-Total - Const. Serv.

TOTAL EQUIPMENT PLUS MATERIAL COSTS

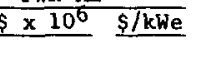

$183 \quad 160$

$$
183
$$

$119 \quad 104$
1986 Constant Dollar

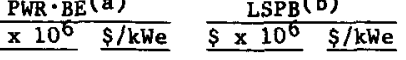

\section{$\frac{\mathrm{HS} 5(\mathrm{c})}{\mathrm{s} \times 10^{6}}$}

118

\begin{tabular}{rr}
- & - \\
- & - \\
29 & 24 \\
17 & 2 \\
12 & 15 \\
11 & 10 \\
-3 & \\
\hline &
\end{tabular}

\begin{tabular}{l}
- \\
- \\
24 \\
20 \\
15 \\
11 \\
10 \\
$\frac{3}{83}$ \\
\hline
\end{tabular}

\begin{tabular}{rr}
19 & 104 \\
- & - \\
- & - \\
26 & 23 \\
17 & 20 \\
12 & 11 \\
11 & \\
3 & \\
\hline
\end{tabular}

104
-
-
23
20
15
11
9
$\frac{3}{81}$

132101

$42 \quad 86$

$\begin{array}{lll}\text { (a) } 1144 \mathrm{MWe} & \text { (b) } 1311 \mathrm{MWe} & \text { (c) } 488 \mathrm{MWe}\end{array}$

\begin{tabular}{rr}
63 & 55 \\
12 & 11 \\
8 & 7 \\
\hline 83 & 73
\end{tabular}

$\begin{array}{r}43 \\ 12 \\ 7 \\ \hline 62\end{array}$

$\begin{array}{r}38 \\ 10 \\ 6 \\ \hline 54\end{array}$

$\begin{array}{cl}- & - \\ - & - \\ 52(\mathrm{e}) & 40(\mathrm{e}) \\ 22 & 17 \\ 17 & 13 \\ 2 & 2 \\ 12 & 9 \\ \frac{2}{107} & \frac{1}{82}\end{array}$

$46 \quad 95$

(c) piptis equipment. Does not include commoditles

(e) The cost of HVAC piping is included in other Equipment for this data model only

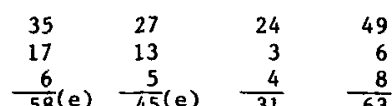

$\begin{array}{llllllll}35 & 31 & 34 & 30 & 40 & 31 & 16 & 33\end{array}$

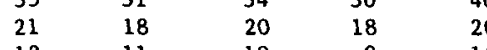

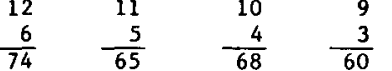

14
$\frac{6}{80}$

$\begin{array}{r}31 \\ 15 \\ 11 \\ 4 \\ \hline 61\end{array}$

16
7
6
2

33
14
11
$-\frac{5}{63}$

$\begin{array}{ll}21 & 18 \\ 22 & 20 \\ 21 & 18\end{array}$

$\begin{array}{r}18 \\ 20 \\ 18 \\ 5 \\ \hline\end{array}$

16
14
16

$\begin{array}{r}16 \\ \quad 4 \\ \hline 50\end{array}$

14
12
14
$\frac{3}{43}$

19
43
15

$\begin{array}{r}15 \\ \quad 4 \\ \hline 81\end{array}$

15
32
12
$\frac{3}{62}$

$40 \quad 35$

43
34
18

18
18

37
29
16
16

$\begin{array}{r}16 \\ 10 \\ \hline 108 \\ \hline\end{array}$

689

\begin{tabular}{rr}
36 & 32 \\
28 & 24 \\
19 & 17 \\
11 & 10 \\
8 & 7 \\
$\frac{6}{73}$ & $\frac{5}{63}$ \\
\hline
\end{tabular}

$58 \quad 44$

$\begin{array}{llll}47 & 36 & 9 & 18\end{array}$

$\begin{array}{rrrr}47 & 36 & 9 & 18 \\ 24 & 18 & 7 & 13 \\ 13 & 10 & 2 & 3 \\ 11 & 8 & 2 & 5 \\ \frac{8}{103} & \frac{6}{78} & \frac{2}{22} & \frac{5}{44}\end{array}$

$\frac{103}{949} \quad \frac{78}{349} \quad \frac{44}{715}$

(f) Indirect Costs

$\begin{array}{ll}1 & 1 \\ r 1 & m \\ 11 & m \\ 11 & m\end{array}$

1
1
11
$1 .:$
1
1
.
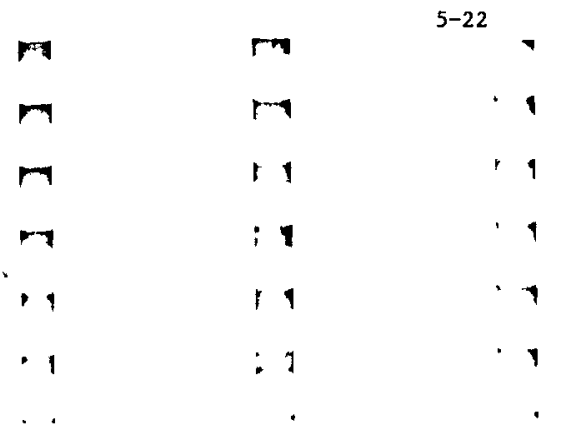

$\begin{array}{ll}m & m \\ m & m \\ m & m \\ m & m \\ m & m\end{array}$




$\begin{array}{ll}E & 11 \\ m & 1 \\ m & 1 \\ 1 & 1\end{array}$

1
5
5
5

$\begin{array}{ll}1 & 1 \\ 1 & 1 \\ r & b \\ r & 1\end{array}$

1:

ENERGY ECONOMIC DATA BASE PHASE VIII UPDATE

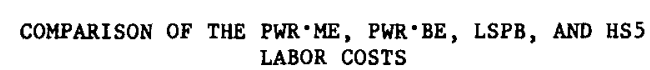

\begin{tabular}{|c|c|c|c|c|c|c|c|c|c|}
\hline & \multirow{2}{*}{\multicolumn{6}{|c|}{ 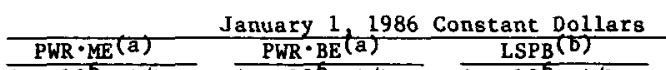 }} & \multicolumn{2}{|c|}{ HSS(c) } \\
\hline & & & & & & & & 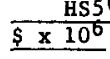 & s/kwe \\
\hline \multirow[t]{5}{*}{ Craft Labor: } & $\begin{array}{c}\text { Structural - Concrete Work } \\
\text { - Other } \\
\text { Sub-Total - Structural }\end{array}$ & $\begin{array}{r}110 \\
\frac{66}{176}\end{array}$ & $\begin{array}{r}96 \\
\frac{58}{154}\end{array}$ & $\begin{array}{r}72 \\
\frac{38}{110}\end{array}$ & $\begin{array}{l}63 \\
\frac{33}{96}\end{array}$ & $\begin{array}{r}78 \\
59 \\
\frac{59}{137}\end{array}$ & $\begin{array}{r}59 \\
\frac{45}{104}\end{array}$ & $\begin{array}{l}\frac{12}{22} \\
\frac{22}{34}\end{array}$ & $\begin{array}{l}24 \\
45 \\
69\end{array}$ \\
\hline & $\begin{aligned} \text { Mechanical } & - \text { P1ping } \\
& - \text { PGD } / \text { Coal/Ash } \\
& - \text { Other } \\
\text { Sub-Tota1 } & \text { Mechanical }\end{aligned}$ & $\begin{array}{l}169 \\
- \\
\frac{57}{226}\end{array}$ & $\begin{array}{l}148 \\
\frac{50}{198}\end{array}$ & $\begin{array}{l}\frac{63}{43} \\
\frac{43}{106}\end{array}$ & $\begin{array}{l}55 \\
-\frac{38}{93}\end{array}$ & $\begin{array}{l}\frac{67}{(d)}^{(\mathrm{d})}{ }^{(\mathrm{d})} \\
144\end{array}$ & $\begin{array}{l}\frac{51(d)}{(d)} \\
\frac{59}{110}{ }^{(d)}\end{array}$ & $\begin{array}{l}22 \\
21 \\
35 \\
78\end{array}$ & $\begin{array}{r}45 \\
42 \\
73 \\
160\end{array}$ \\
\hline & $\begin{aligned} & \text { Electrical/I } \& C- \text { Raceway } \\
&- \text { Other } \\
&- \text { Cable } \\
& \text { Sub-Total - Electrical/I } \& C\end{aligned}$ & $\begin{array}{r}36 \\
30 \\
21 \\
87\end{array}$ & $\begin{array}{l}31 \\
27 \\
18 \\
76\end{array}$ & $\begin{array}{l}16 \\
25 \\
11 \\
52\end{array}$ & $\begin{array}{l}14 \\
21 \\
10 \\
45\end{array}$ & $\begin{array}{l}18 \\
38 \\
13 \\
69\end{array}$ & $\begin{array}{l}14 \\
29 \\
10 \\
53\end{array}$ & $\begin{array}{r}8 \\
9 \\
4 \\
21\end{array}$ & $\begin{array}{r}16 \\
19 \\
8 \\
43\end{array}$ \\
\hline & Construction Services (e) & 188 & $\underline{164}$ & 97 & 85 & 124 & 95 & 18 & 38 \\
\hline & Sub-Total - Craft Labor & 677 & 592 & 365 & 319 & 474 & 362 & 151 & 310 \\
\hline Salaries:(e) & $\begin{array}{l}\text { Engineering } \\
\text { Field Job Supervision } \\
\text { Other } \\
\text { Sub-Total - Salaries }\end{array}$ & $\begin{array}{l}180 \\
202 \\
\frac{31}{413}\end{array}$ & $\begin{array}{l}157 \\
177 \\
\frac{27}{361}\end{array}$ & $\begin{array}{r}78 \\
40 \\
13 \\
131\end{array}$ & $\begin{array}{r}68 \\
35 \\
12 \\
115\end{array}$ & $\begin{array}{r}106 \\
52 \\
17 \\
175\end{array}$ & $\begin{array}{r}81 \\
40 \\
13 \\
134\end{array}$ & $\begin{array}{r}10 \\
10 \\
2 \\
22\end{array}$ & $\begin{array}{r}21 \\
2 i \\
-3 \\
45\end{array}$ \\
\hline Expenses: $(e)(f)$ & $\begin{array}{l}\text { Engineering } \\
\text { Fie1d Job Supervision } \\
\text { Other } \\
\text { Sub-Tota1 - Expenses }\end{array}$ & $\begin{array}{l}267 \\
147 \\
\frac{32}{446}\end{array}$ & $\begin{array}{l}233 \\
129 \\
\frac{27}{389}\end{array}$ & $\begin{array}{r}115 \\
30 \\
15 \\
160\end{array}$ & $\begin{array}{r}101 \\
26 \\
13 \\
140\end{array}$ & $\begin{array}{r}157 \\
39 \\
20 \\
216\end{array}$ & $\begin{array}{r}120 \\
29 \\
\frac{15}{164}\end{array}$ & $\begin{array}{r}16 \\
7 \\
-2 \\
25\end{array}$ & $\begin{array}{r}32 \\
15 \\
4 \\
51\end{array}$ \\
\hline Insurance/Taxes: (e) & Sub-Total - Insurance/Taxes & $s \underline{114}$ & 100 & 62 & 54 & 81 & 61 & $\underline{26}$ & 53 \\
\hline TOTAL LABOR COSTS & & 1650 & 1442 & 718 & 628 & 946 & 721 & 224 & 459 \\
\hline $\begin{array}{l}\text { TOTAL EQUIPMENT PLUS } \\
\text { (FROM TABLE 5-11) }\end{array}$ & MATEE & 788 & 689 & 683 & 597 & $\underline{1244}$ & 949 & 349 & 715 \\
\hline TOTAL & ION CC & 2438 & 2131 & 01 & 1225 & 190 & 670 & 573 & 174 \\
\hline
\end{tabular}

$\begin{array}{lll}\text { (a) } 1144 \mathrm{MWe} & \text { (b) } 1311 \mathrm{MWe} & \text { (c) } 488 \mathrm{MWe}\end{array}$

(a) The labor cost for Installation of HVAC piping 18 1ncluded with "Mechanical - other" for this data model only
(d) Indirect costs

(f) Includes payroll expenses, overhead loading, fees, outside consultants, and relocation and other expenses 


\section{SECTION 6}

\subsection{REVIEW OF ADVANCED LWR CONCEPTS FOR THE 1990 'S AND BEYOND}

\subsection{INTRODUCTION}

During the Phase VII (1984) Update, a review of developing nuclear power plant design concepts and practices was initiated. The focus of the review was to determine the directions that plant designs for the $1990^{\prime}$ s and beyond might take. The objective of the review was to identify what changes should be made in future EEDB updates of the PWR BE or a modification of the PWR BE to reflect the potential base construction costs of future nuclear power plants.

The review was extended into the Phase VIII Update because much of the advanced work being done was still in a preliminary or formative stage by January 1 , 1984, the cost and regulation date of the Phase VII Update. During the Phase VIII Update, preliminary results of the ongoing work began to become available. The following sections give a brief overview of the ongoing activities and the early results of some of these activities.

\subsection{POTENTIAL DESIGN FEATURES AND PRACTICES FOR FUTURE NUCLEAR POWER PLANTS}

A variety of organizations are working on the development of improved design features and design/construction/operation/maintenance practices that may be applied to current (operating or under construction) and future nuclear power plants. The objective of these efforts is to improve nuclear power plant safety, costs, availability, maintainability and operability. The major nuclear industry groups and their significant areas of investigation are as follows:

Institute of Nuclear Power Operations (INPO): This utility supported group has developed a managed event analysis program and various other programs, such as the National Academy for Nuclear Training, that provide guidance and data for the improvement of nuclear power plant operations and maintenance and related design modifications.

Electric Power Research Institute (EPRI): This utility supported institute is sponsoring development of a requirements document for future nuclear power plants and, with USDOE, conceptual designs for small advanced light water reactor nuclear power generating stations. These two activities are a part of the EPRI Advanced Light Water Reactor (ALWR) Program. EPRI is also developing guidance on operations and maintenance activities and equipment qualification testing and maintenance through their good practice guide program.

The Nucleax Utility Management and Resources Committee (NUMARC): This committee is composed of senior executive and management representatives from all nuclear utilities. Its purpose is to conduct an integrated review of management and other issues related to nuclear power plant operation or construction. Among its current areas of oversight are maintenance activities and the station blackout issue. 
The Seismic Qualification Utility Group (SQUG): This group is composed of utility representatives from most nuclear utilities. It has investigated the effects of earthquakes on operating conventional power plant equipment and assembled data to permit seismic qualification of certain nuclear power plant equipment by experience, one of three acceptable methods (the other two are test and analysis).

The American Nuclear Society (ANS) Special Committee on Source Terms: This committee, with the assistance of the NRC, USDOE and EPRI, investigated the ramifications of the TMI-2 incident on the methodologies for predicting source terms. Their findings will most likely have a significant impact on future nuclear power plant design features. In the wake of the Chernobyl incident, however, the study results will probably be reviewed to assure that the impact of any additional lessons to be learned are included.

U.S. Department of Energy Three-Mile Island (TMI) Research and Development Program: The objectives of the TMI element are to develop the technology that will improve the capability of civilian nuclear power plants to safely recover from accidents and to conduct relevant research and development that will substantially enhance nuclear power plant safety and operation. There are two primary subelements within this activity: (1) TMI Waste Immobilization; and (2) Reactor Evaluation and Data Acquisition Program.

All of these activities are producing data that will be useful and needed in developing a viable and competitive generation of nuclear power plants for the future. It is likely that the EPRI requirements document and the EPRI/DOE ALWR program will have the most significant impact on future units for the near term. These efforts are so comprehensive that they may prove to be the focal point for the incorporation of findings from the other areas into future plant designs.

\subsection{U.S. ADVANCED LWR PROGRAM}

The Electric Power Research Institute is currently sponsoring, with assistance from the U.S. Department of Energy, the basic elements of a U.S. Advanced LWR Program. This program has the following activities and responsibilities:(17)

1. Determine the set of regulatory requixements for the next generation LWR (EPRI).

2. Generate utility-approved NRC certified plant requirements documents for advanced LWR nuclear power plants (EPRI).

3. Develop detailed designs and final licensing certification of advanced LWR nuclear power plants (DOE).

4. Develop designs of a small ( $\leq 600$ MWe) advanced PWR and BWR (EPRI-design, DOE-hardware development). 
The first element of work (Item 1) has been completed in conjunction with the NRC, while the other elements are currently in progress. The results of this work are being used as guidance for the remaining elements.

The requirements document is composed of 13 chapters which will provide a comprehensive set of criteria and requirements for the design of a nuclear power plant when completed. A utility steering committee provides utility input on desired approaches and numerical goals for various requirements. When completed, each chapter will be reviewed by industry (utilities, manufacturers and architect-engineers) and the NRC. The contents of the 13 chapters are proposed to be as follows:

1. Overall Requirements

2. Power Generation

3. Primary Coolant System and NonSafety Auxiliary Systems

4. Reactor Systems

5. Safety Systems

6. Arrangements

7. Fueling and Refueling

\section{Plant Cooling Water Systems}

9. Site Support Systems

10. Plant Instrumentation and Control

11. Electric Power Systems

12. Radioactive Waste Processing Systems

13. Turbine Generator Systems

Chapter 1 , the Overall Requirements, sets enveloping requirements for general design, design basis events, structural design basis, materials, reliability and availability, constructability, operability and maintainability, quality assurance, and licensing. The current status of the requirements document is that Chapter 1 is complete and Chapter 2 is under review.

The objective of the EPRI small advanced PWR/BWR design development is to develop a conceptual design for a 600 MWe or smaller nuclear power plant based on current technology, but having advanced design features that permit it to be a viable option for the future. This effort has been under way for about 18 months and will continue for an additional 42 months. DOE is sponsoring various supporting projects related to such topics as passive safety systems and constructability. From the standpoint of the EEDB Program objectives, this activity promises to be one of the most useful source of advanced design/practice data for future updates.

\subsection{ADVANCED LWR NUCLEAR POWER PLANT TECHNICAL DESIGN FEATURES}

It is expected that medium capacity light water reactor nuclear power generating station (LWR) designs for the 1990's and beyond will be based on the extensive body of existing U.S. technology and experience. The first of the future medium capacity LWR (LWR $\cdot F$ ) designs will most likely be advanced modifications of the current technology, based on the lessons learned from 
current and past experience. If there are to be LWR $F^{\prime} s$, they will need to be pexceptually safer, simpler and more forgiving in design, able to be built more quickly and cheaply, and easier to maintain and operate. The EPRI ALWR Requirements Document cites the following as preferred design characteristics:

Enhanced Safety

No Licensing Uncertainty

Enhanced Operability

Enhanced Maintainability

Enhanced Availability

60 Year Life

Increased Design Margins
Simplified Plant

Reduced Waste Disposal

Proved Systems/Components

Existing Problems Minimized

Short Construction Schedule

Cost Competitive

\subsubsection{Regulatory Considerations for the LWR $F$}

The safety/licensing objectives for the LWR $F$ will most assuredly not change from those of today. The LWR $F$ will need to be designed to preserve public safety, minimize occupational exposure and follow the defense-in-depth concept. Nevertheless, some kind of regulatory reform, similar to the bills currently being considered by congress, will be required to make the regulatory process less time consuming and more predictable. The combined construction and operating license ( $\mathrm{COL}$ ) concept and the requirement to introduce additional material facts before a settled issue may be reopened are examples of the kind of reforms that can meet these objectives.

Current regulations are applicable to LWR's that are in the 800 to 1250 MWe capacity range and that depend on active safety systems to meet the above safety/licensing objectives. The LWR $\cdot F$ is likely to depart from this norm because the medium sized advanced plants being considered have nominal capacities of 600 MWe and rely to a great extent on passive safety features and inherent safety. It will be necessary to carefully review the current body of regulations to ascertain their suitability for application to medium to small advanced plants. Where their application would unnecessarily dilute the unique advantages of the advanced design features, alternatives for the general design criteria and design review bases would need to be developed. The alternatives would have to specifically address the medium to small capacity advanced plant design features, while assuring their conformance to the basic safety objectives.

\subsubsection{Advanced Design Feature Objectives for the LWR •F}

In order to provide the preferred design characteristics listed above, the advanced plants under development are attempting to meet a variety of design feature objectives. Table 6-1 lists some of the major design objectives for the nuclear steam supply system. Table 6-2 lists some of the major design objectives for the balance of plant systems and structures. An overall objective is enhanced constructability that is based on modularity, ease of transportation and ease and speed of field assembly. Many of these design objectives relate to or may be achieved by plant simplification. 
Incorporation of the findings of various industry organizations such as INPO and SQUG can lead to simplification of complex systems and structures. The simplification can lead to greater safety, shorter construction schedules, lower cost, and higher maintainability, operability and availability. The objective of a 60 year plant life will most likely be met by designs that include 60 year life reactor vessels and provisions to replace everything else, including the steam generators for the PWRs. A detailed description of each preliminary concept may be found in Reference 18 .

One of the major attributes of the advanced plant designs is that their design objectives, if met, will reduce labor hours and associated costs. For current plants under construction, labor (craft, engineering and field supervision) costs have been the fastest rising cost components since 1978. In fact, rising labor costs have been the major reason that nuclear power has lost its competitive edge to coal-fired power plants. Plant simplification, reduction in Category I structures, reducing the area within the safety related boundary of the plant and the incorporation of passive safety features will significantly reduce both craft and engineering hours related to current plant best experience. Reduction in craft and engineexing hours will reduce field supervision hours, because there will be fewer craftsmen to supervise and fewer engineering activities with which to interface.

\subsection{ADVANCED LWR NUCLEAR POWER PLANT POTENTIAL COST AND SCHEDULES}

\subsubsection{LWR $\cdot \mathrm{F}$ Cost and Schedule}

The advanced plants appear to have the potential to achieve construction schedules of 48 months (an EPRI ALWR Program requirement) with overall plant licensing/engineering/construction schedules of about 70 months. An abbreviated estimated schedule was developed for an LWR F based on EPRI ALWR and DOE EEDB Program requirements and data. Table 6-3 compares this schedule with those of the EEDB PWR BE and HS5. The LWR F construction schedule is based on the plant simplification achieved in meeting the NSSS and BOP design objectives discussed above. The nth-of-a-kind LWR $F$ licensing/engineering schedule is based on a fifth-of-a-kind or higher plant with a standard design. For this scenario, only site related differences that are outside of the base design envelope require licensing or engineering attention.

The advanced plants also appear to have the potential to achieve base construction costs that permit nuclear power plants to be competitive with coal-fired power plants. A rough estimate of the base construction cost was developed for an LWR $F$ based on EPRI ALWR and DOE EEDB Program requirements and data. Table 6-4 compares the LWR $\cdot F$ potential base construction costs with those of an EEDB PWR BE and HS5. The LWR $\cdot F$ costs are based on the plant simplification and short schedule discussed above and the resulting reduction in craft, engineering and field supervision hours, particularly the engineering hours. 


\subsubsection{LWR F Advantages Relative to a Large PWR}

If the NSSS and BOP design objectives given in Tables 6-1 and 6-2 can be met for the $L W R \cdot F$, a number of advantages are realized. A medium capacity nuclear power plant becomes available that is about the same cost (on a $\$ / \mathrm{kWe}$ basis) as the current best experience large PWR's and only about four percent more expensive than an equivalent sized coal-fired power plant, as represented by the EEDB HS5. The capital expenditure (in dollars) is only about half that for the large PWR and 11 percent higher than that for a coal-fired power plant of 600 MWe capacity. Additionally, the construction schedule is about 67 and 112 percent of the large PWR and the median capacity coal-fired power plant, respectively. Relative to the large PWR, the LWR F would be a bettex capacity match to currently projected growth and overall radiation exposure to personnel would be lower because of the improved and advanced design features. Also, based on plant simplification and reduction in the number of Seismlc Category I structures and safety class systems, improvements could occur in safety, maintainability, operability and availability. Consequently, it appears that a combination of the current technology base with appropriate advanced design features and plant simplification could hold the promise of a viable future for nuclear power. 
TABLE 6-1

ENERGY ECONOMIC DATA BASE

PHASE VIII UPDATE

NSSS ADVANCED DESIGN FEATURE OBJECTIVES FOR THE LWR F

- Extend Life of Reactor Vessel [60 Years(a)]

- Increase Primary System Water Inventory

- Eliminate Reactor Coolant Pump Seals

- Enhance Natural Circulation of Reactor Coolant

- Provide Passive Core Flooding

- Provide Passive Decay Heat Removal

- Provide Passive Safety Injection

(a) Based on EPRI ALWR Requirements Document 
TABLE 6-2

ENERGY ECONOMIC DATA BASE

PHASE VIII UPDATE

BOP ADVANCED DESIGN FEATURE OBJECTIVES FOR THE LWR $\cdot$ F

- Reduce Extent of Plant Safety-Class Boundary

- Reduce Seismic Category I Building Volume

- Provide Passive Containment Pressure/Temperature Control

- Provide Passive Containment Cooling

- Provide Improved Fuel Handling

- Refueling Interval 18-24 Months(a)

- Down Time 45-60 Days(a)

- Simplify Systems and Equipment

- Reduce Occupational Radiation Exposure and Off-Site Radwaste Shipping Volumes

- Improve Turbine Plant Systems and Equipment

- Improve Electrical and I\&C Systems and Equipment

- Reduce In-Service Inspection

- Provide for Replacing Major Components (Including Steam Generators)

(a) Based on EPRI ALWR Requirements Document 
TABLE 6-3

ENERGY ECONOMIC DATA BASE

PHASE VIII UPDATE

POTENTIAL CONSTRUCTION SCHEDULES FOR THE LWR - F AS COMPARED WITH THE EEDB PWR B BE AND THE EEDB HS5

Duration (Months)

\begin{tabular}{lcc}
\multicolumn{3}{c}{ Duration (Months) } \\
\hline LWR $\cdot$ F & & \\
Nth-of- & EEDB & EEDB \\
a-kind & PWR $\cdot$ BE & HS5 \\
600 MWe & $1144 \mathrm{MWe}$ & $488 \mathrm{MWe}$ \\
\hline
\end{tabular}

Plant Specific Engineering and Licensing,

18

42

42

and Site Development

Construction (First Structural Concrete

$48(a)$

72

43

Pour to Fuel Load)

startup (b)

$\underline{4}$

70

120

88

(a) Based on EPRI ALWR Requirements Document

(b) Start-up tests and checks actually start when electric power is aval1able, and is conducted in parallel with other work, prior to fuel load (nuclear) or commercial operation (fossil). 
TABLE 6-4

\section{ENERGY ECONOMIC DATA BASE}

PHASE VIII UPDATE

$\mathrm{LWR} \cdot \mathrm{F} / \mathrm{PWR} \cdot \mathrm{BE} / \mathrm{HS} 5$

BASE CONSTRUCTION COST COMPARISON

Account

Total Direct Costs

Total Indirect Costs

Base Construction Costs

\$/kWe
Constant $\$ \times 10^{6}(1 / 86)$

\begin{tabular}{|c|c|c|}
\hline $\begin{array}{l}\mathrm{LWR} \cdot \mathrm{F} \\
\mathrm{n} \text { th-of- } \\
\text { a-kind } \\
600 \text { MWe }\end{array}$ & $\begin{array}{c}\text { EEDB } \\
\mathrm{PWR} \cdot \mathrm{BE} \\
1144 \mathrm{MWe}\end{array}$ & $\begin{array}{c}\text { EEDB } \\
\text { HS5 } \\
488 \text { MWe }\end{array}$ \\
\hline 514 & 878 & 460 \\
\hline 219 & 523 & 113 \\
\hline 733 & 1401 & 573 \\
\hline 1222 & 1225 & 1174 \\
\hline
\end{tabular}


$\underline{\text { SECTION } 7}$

7.0 REFERENCES, DEFINITIONS AND GLOSSARY

\subsection{REFERENCES AND BIBLIOGRAPHY}

1. "Final Report and Initial Update of the Energy Economic Data Base (EEDB) Program - Phase I," United Engineers \& Constructors Inc., Philadelphia, PA 19101, UE\&C/ DOE-790930, U.S. Department of Energy Contract EN-78-C-02-4954, December, 1979.

2. "Phase II Final Report and Second Update of the Energy Economic Data Base (EEDB) Program," United Engineers \& Constructors Inc., Philadelphia, PA 19101, UE\&C/DOE -810430 , U.S. Department of Energy Contract DE-ACO2-78ET33020, (former1y EN-78-C -02-4954), Apri1, 1981 .

3. "Phase III Final Report and Third Update of the Energy Economic Data Base (EEDB) Program," United Engineers \& Constructors Inc., Philadelphia, PA 19101, UE\&C/DOE -810731 , U.S. Department of Energy Contract DE-AC02-78ET33020, (former1y EN-78-C $-02-4954)$, July, 1981 .

4. "Phase IV Final Report and Fourth Update of the Energy Economic Data Base (EEDB) Program," United Engineers \& Constructors Inc., Philadelphia, PA 19101, UE\&C/DOE -810930 , U.S. Department of Energy Contract, (Argonne National Laboratory) 31109-38-6411, September, 1981; and Supplement, November, 1981 .

5. DOE/NE-0051 "Phase V Update (1982) Report for the Energy Economic Data Base Program EEDB-V," prepared for the U.S. Department of Energy by United Engineers \& Constructors Inc., Philadelphia, PA 19101, under Contract No. CH-ENG-38-6818, July, 1983 .

6. DOE/NE-0051/1 "Phase VI Update (1983) Report for the Energy Economic Data Base Program - EEDB VI," prepared for the U.S. Department of Energy by United Engineers \& Constructors Inc., Philadelphia, PA 19101, under Contract No. DE-AC05840R21400, September, 1984 .

7. DOE/NE-0051/2 "Phase VII Update (1984) Report for the Energy Economic Data Base Program EEDB-VII," prepared for the U.S. Department of Energy by United Engineers \& Constructors Inc., Philadelphia, PA 19101, under Contract No. DE-AC05840R21400, August, 1985 .

8. DOE/NE-0052 "Program Reference Book for the Energy Economic Data Base Program EEDB," prepared for the U.S. Department of Energy by United Engineers \& Constructors Inc., Philadelphia, PA 19101, under Contract No. CH-ENG-38-6818, July, 1983 .

9. DOE/NE-0059/1, "Technical Reference Book for the Energy Economic Data Base Program - EEDB," prepared for the U.S. Department of Energy by United Engineers \& Constructors Inc., Ph1ladelphia, PA 19101, under Contract No. DE-AC05-840R21400, August, 1985. 
10. "Energy Economic Data Base (EEDB) Program, EEDB Program Technical Reference Book, Phase VIII Update (1986)," United Engineers \& Constructors Inc., Philadelphia, PA 19101, UE\&C/ORNL-860731, Martin Marietta, Subcontract No. 43X$86004 \mathrm{~V}, \mathrm{July}, 1986$.

11. DOE/NE-0059 "Technical Reference Book for the Energy Economic Data Base Program - EEDB," prepared for the U.S. Department of Energy by United Engineers \& Constructors Inc., Philadelphia, PA 19101, under Contract No. DE-AC05-840R21400, September, 1984 .

12. "Energy Economic Data Base (EEDB) Program, Phase VIII Update (1986) Report BWR Supplement," United Engineers \& Constructors Inc., Philadelphia, PA 19101, UE\&C/ORNL-860930-B, Martin Marietta Subcontract No. 43X-86004V, September, 1986.

13. "A Compilation of Federal Regulations for the Design and Licensing of Power Plants Including Engineering Guidelines for Their Implementation," prepared by United Engineers \& Constructors Inc., Philadelphia, PA 19101, December, 1973 and Revisions through September, 1985 (Controlled Distribution).

14. "Commercial Electric Power Cost Studies," United Engineers \& Constructors Inc., NUREG: U.S. Nuclear Regulatory Commission and/or COO: U.S. Energy Research and Development Administration.

a. "Capital Cost: Pressurized Water Reactor Plant," Volumes 1 and 2 , NUREG-0241, C00-2477-5, June 1977.

b. "Capital Cost: Low and High Sulfur Coal Plants - 800 MWe (Nominal)" Volumes 1, 2 and 3, NUREG-0244, C00-2477-8, June, 1977.

15. "Large Scale Prototype Breeder," (Document Number CDS 100-2, Revision 7, 2/84, Overall Plant Design Specification et seq.) prepared for the U.S. Department of Energy by Consolidated Management office for the LMFBR of the Electric Power Research Institute, September, 1984.

16. R. A. Lindley and J. E. Stader, "American Designers Achieve Reduced Costs," Nuclear Engineering International, July, 1986, pp. 31-33.

17. Karl E. Stahlkopf et al, ALWR Program - EPRI, "Next Generation LWR's," presented at ANS/ASME Nuclear Power Conference, July 21, 1986.

18. Nuclear Europe, March 1986:

a. D. Wilkins et al, "Advanced Boiling Water Reactor Plant," pp. 11-15.

b. R. A. George, "Advanced PWR: New Reactor Design for Next Decade," pp. 15-17.

c. G. E. Kulynych and J. E. Lemon, "Babcock \& Wilcox Advanced PWR Development," pp. 17-19. 


\subsection{DEFINITIONS}

The following commonly used terms are specifically defined as they are used in the Energy Economic Data Base Program.

\subsubsection{Rated Load (Unit or Equipment)}

The operating condition that exists when the unit steam turbine-generator is operating at that load for which the turbine-generator performance is guaranteed by the manufacturer.

\subsubsection{Rated Thermal Power (Nuclear Power Generating Stations)}

The rated load heat input to the reactor coolant from the reactor core expressed as thermal megawatts (MWt).

\subsubsection{Rated Thermal Power (Fossil Power Generating Stations)}

The rated load heat input to the boiler in the form of fuel, expressed as thermal megawatts (MWt).

\subsubsection{Net Power to GSU (Generator Step-up Transformer)}

The generator output at rated load and voltage reduced by the plant auxiliary power load at these conditions, expressed as electric megawatts (MWe).

\subsubsection{Net P lant Heat Rate}

The figure of merit obtalned by dividing the product of the "Thermal Power" and the constant 3412.14 (British thermal units per hour per electric kilowatt) by the "Net Power to GSU," expressed as British thermal units/kilowatt-hour (Btu/kWh).

\subsubsection{Net Plant Efficiency}

The figure of merit obtained by dividing the "Net Power to GSU" by the "Thermal Power," expressed as a percentage.

\subsubsection{Maximum Continuous Rating (Fossil Boiler)}

The boiler design condition which corresponds to a main and reheat steam pressure of five percent above that for rated load conditions, and a flow rate in excess of that predicted by the turbine manufacturer at the valves wide open, five percent overpressure condition.

\subsubsection{Valves Wide Open, Five Percent Overpressure Condition (VWO, 5\% OP)}

The operating condition that exists when the turbine is operated with the main throttle valve(s) fully opened and the main steam pressure at the throttle valve inlet five percent greater than that at rated load. Typically, this is 
the maximum load condition for which the turbine manufacturer predicts (but does not guarantee) performance.

\subsubsection{Base Construction Cost}

The base construction cost is the overnight plant cost for the direct and indirect costs only. It does not include contingency, owner's cost, allowance for funds used during construction, initial supply of fuel nor other costs related to utility preference or specific utility system requirements (Refex to Table 5-2).

\subsubsection{Constant Dollars}

Constant dollar cost is the cost which would be paid for an item or a service in the future if there were no inflation between the time that the cost is estimated and the time the cost is incurred.

\subsubsection{Site Materials}

Site materials are field purchased or bulk commodity items. Piping two inches in diameter and smaller nominal pipe size is site material. All galvanized pipe, storm, roof and floor drainage piping and sanitary piping are also site material. Wire/cable and raceways, including those in building service lighting and power systems, are site material.

\subsubsection{Factory Equipment}

Factory equipment is manufactured or factory fabricated items. Piping 2.5 inches and larger is factory equipment, with the exception of galvanized pipe, storm, roof and floor drainage piping and sanitary piping.

\subsubsection{Force Account}

Force account refers to the direct hiring and supervision of craftsmen by a prime contractor to perform a construction task.

\subsubsection{Subcontract Account}

Subcontract account refers to the hiring of a subcontractor by a prime contractor to perform a construction task in accordance with appropriate specifications. 


\subsection{GLOSSARY OF ACRONYMS AND ABBREVIATIONS}

This glossary contains acronyms and abbreviations that are applicable to the EEDB Program Update Reports and Technical Reference Books.

\subsubsection{Governmental Organizations}

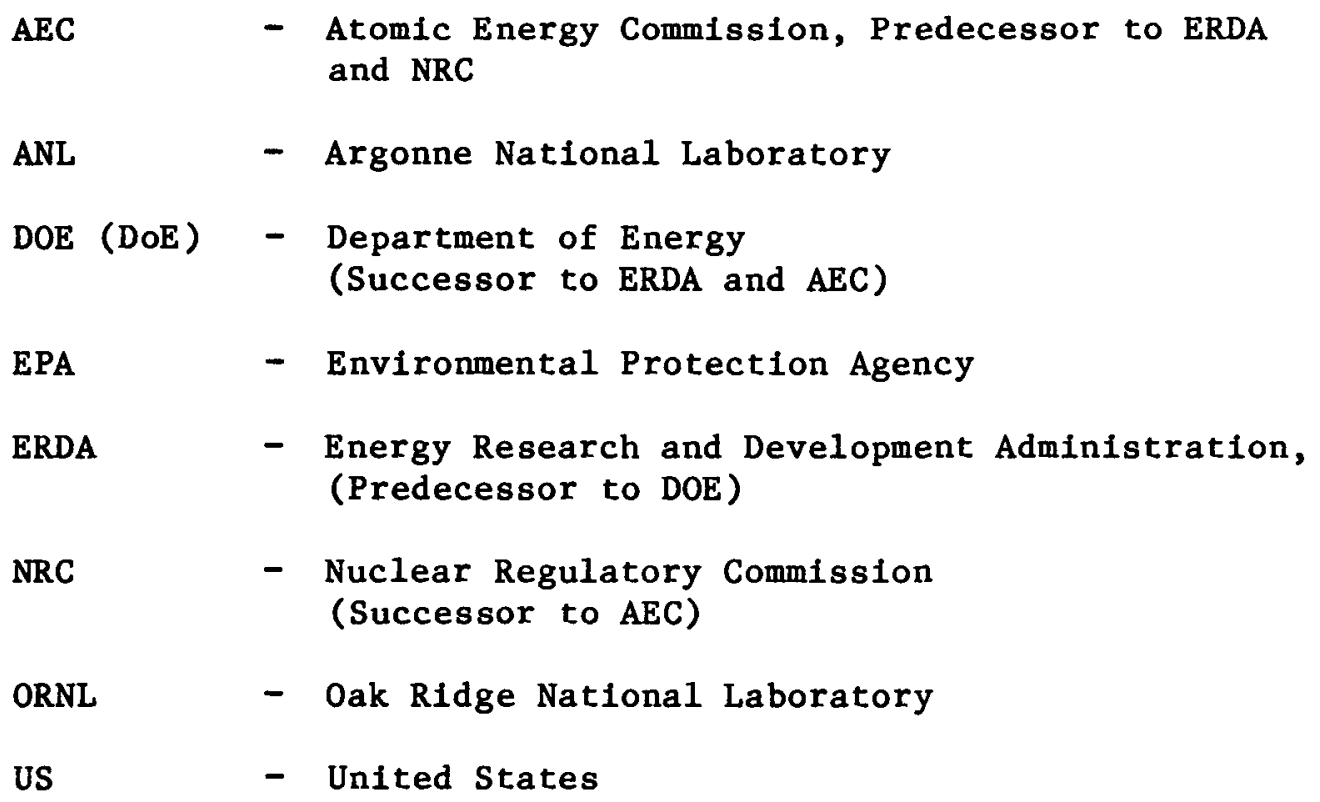

\subsubsection{Other Organizations}

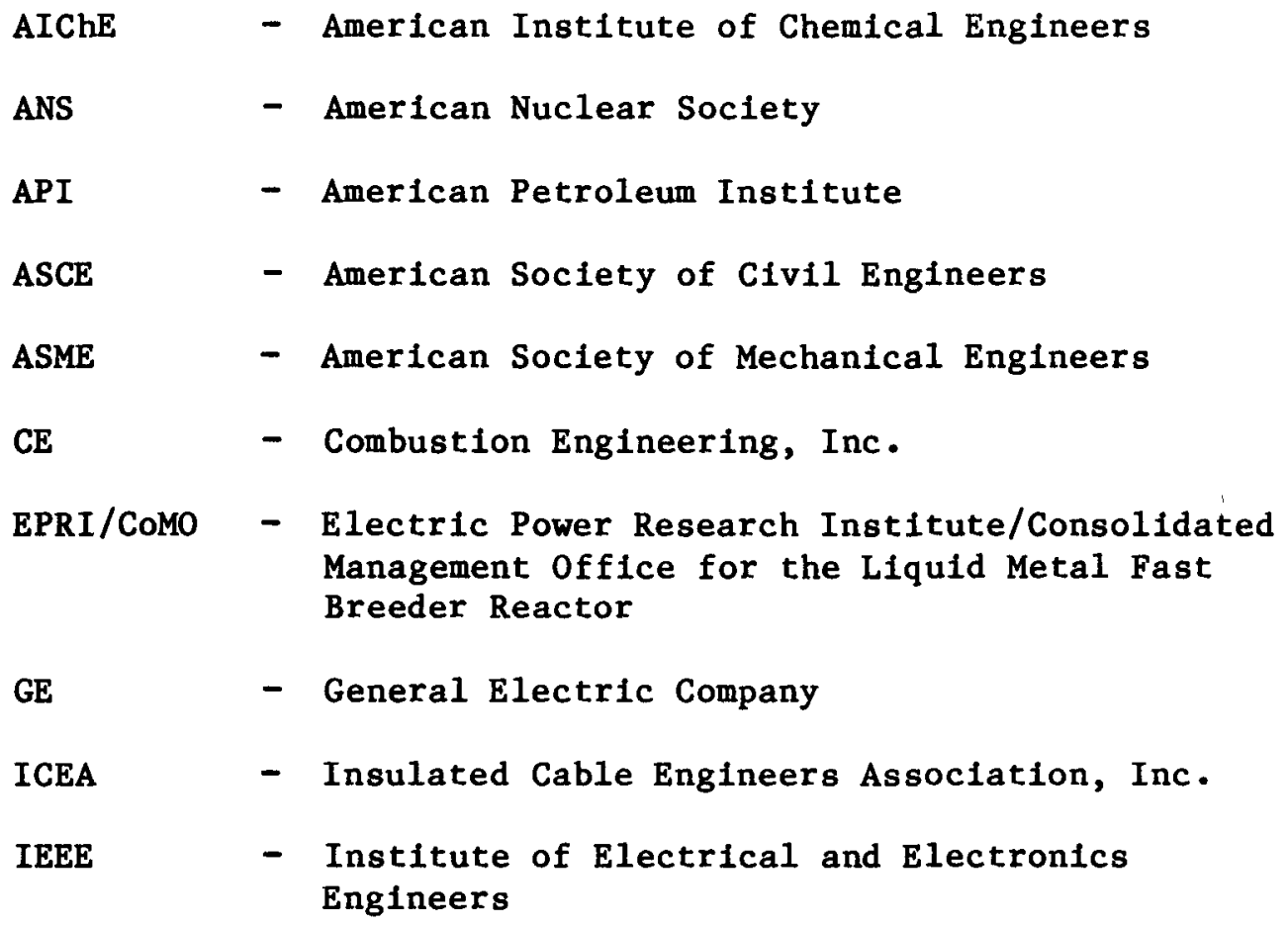




\subsection{2 (Cont'd)}

$\begin{array}{ll}\text { ISA } & - \text { Instrument Society of America } \\ \text { NFPA } & - \text { National Fire Protection Association } \\ \text { UE\&C } & -\begin{array}{l}\text { United Engineers \& Constructors Inc. } \\ \text { (A Raytheon Subsidiary) }\end{array} \\ \text { UL } & - \text { Underwriters' Laboratories, Inc. } \\ \text { WE } & - \text { Westinghouse Electric Corporation } \\ \text { WEC } & \end{array}$

\subsubsection{Technical/Cost Identification and Programs}

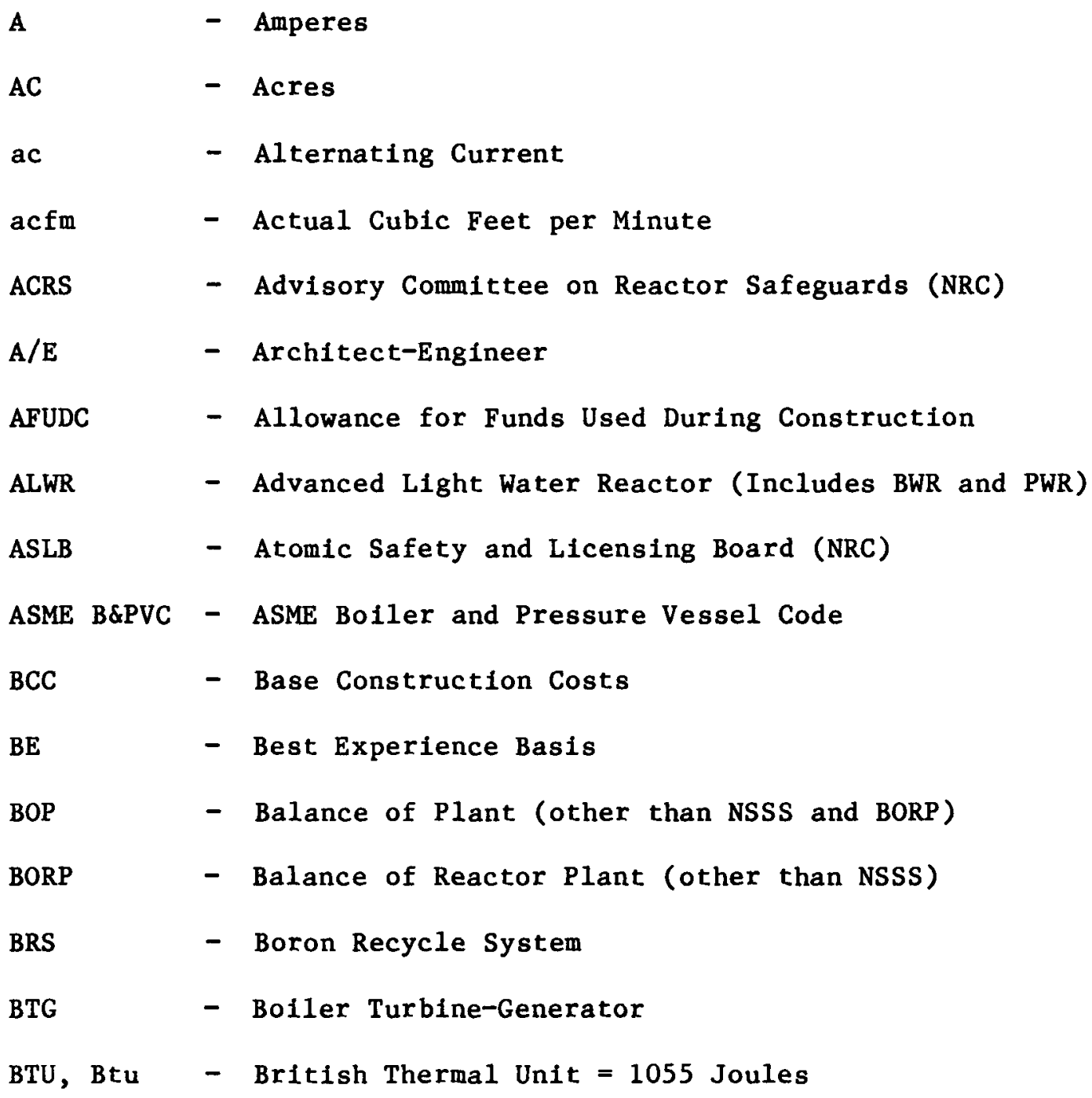




\subsection{3 (Cont'd)}

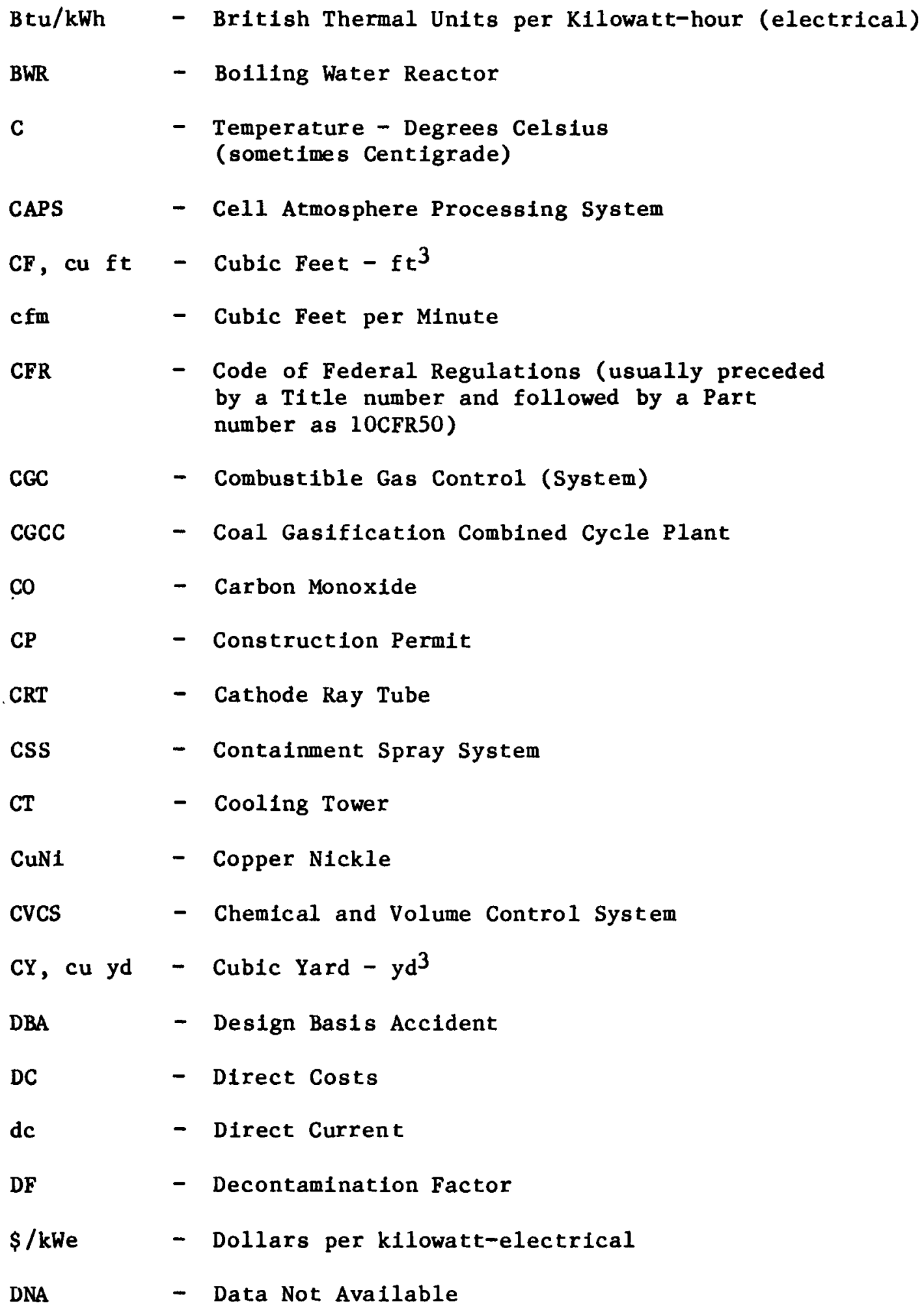




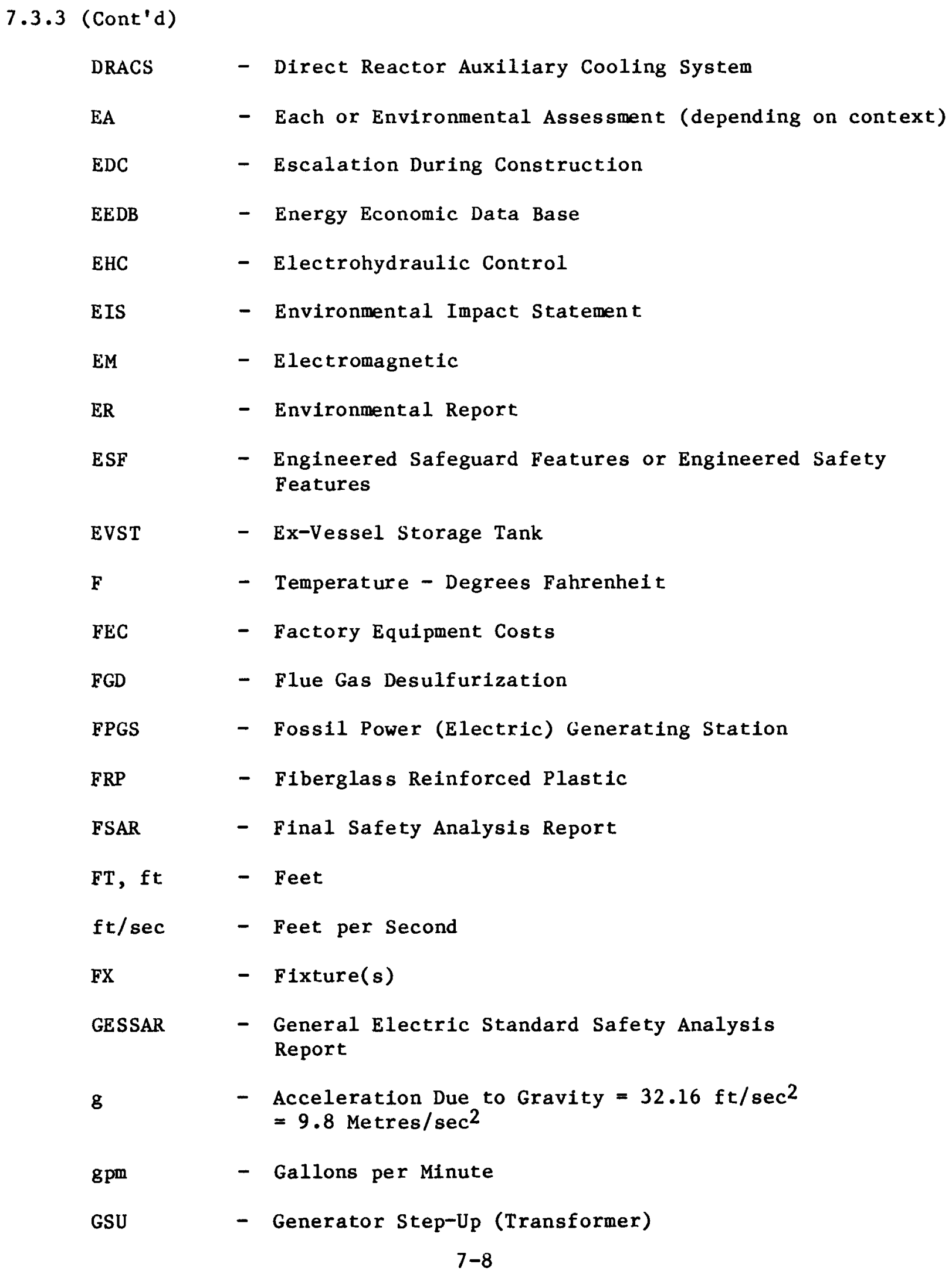




\section{3 .3 (Cont'd)}

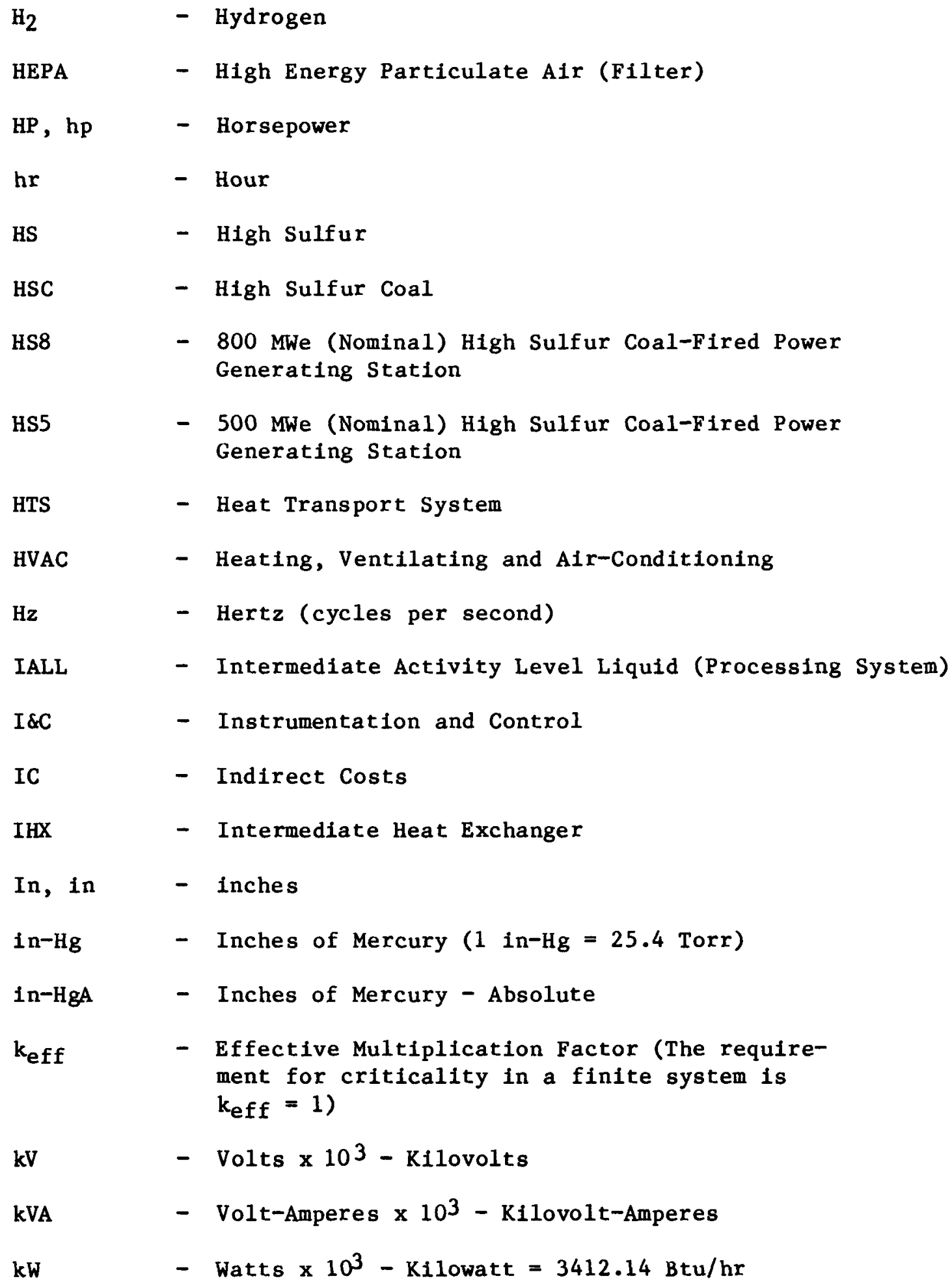


7.3.3 (Cont'd)

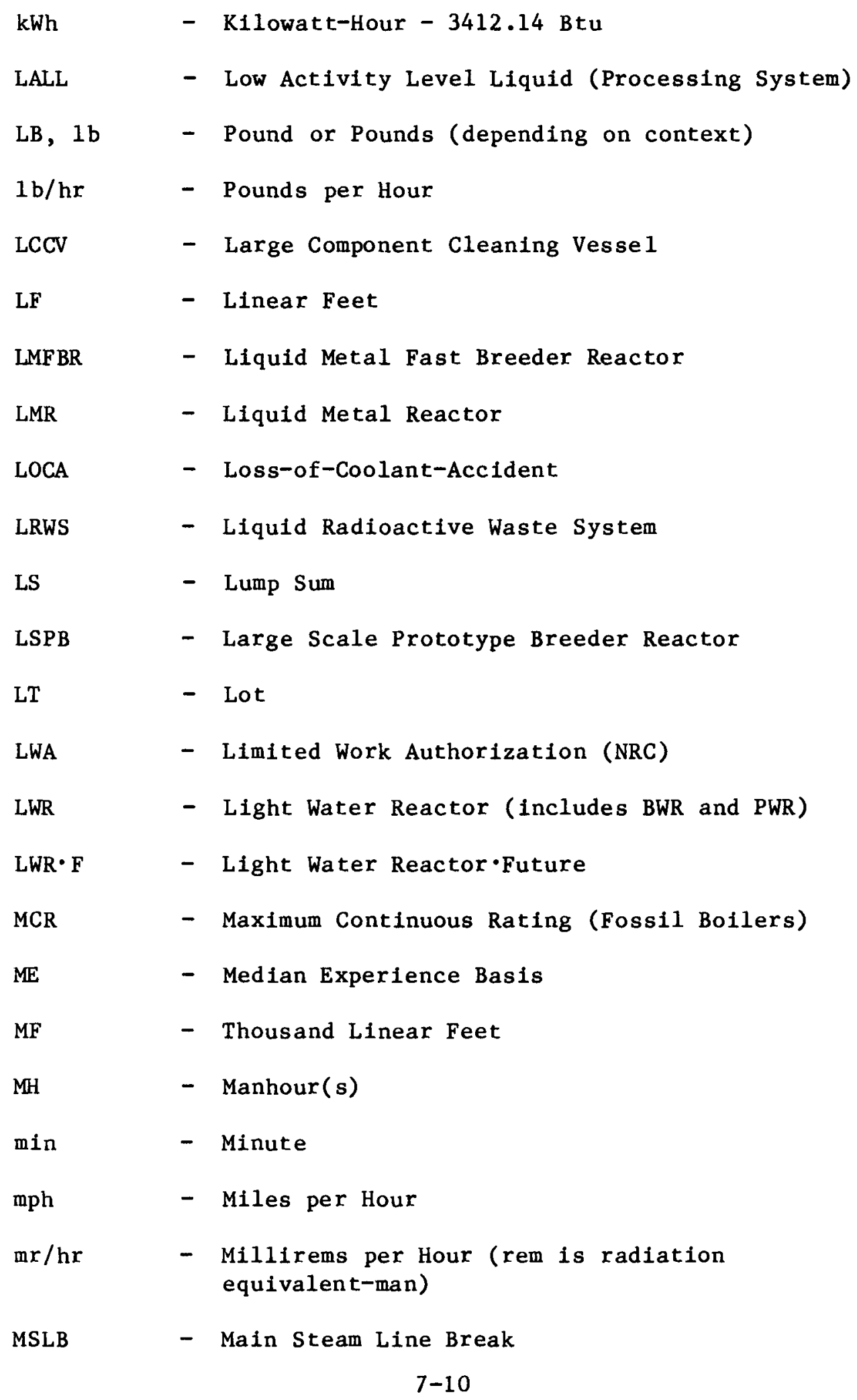


7.3.3 (Cont'd)

\begin{tabular}{|c|c|c|}
\hline MVA & - & Volt-amperes $\times 10^{6}$ - Megavolt-amperes \\
\hline MW & - & Watts $\times 10^{6}$ - Megawatt \\
\hline MWe & - & Megawatts - Electrical \\
\hline MWt & - & Megawatts - Thermal \\
\hline N/A & - & Not Applicable \\
\hline $\mathrm{N} / \mathrm{Av}$ & - & Not Available \\
\hline $\mathrm{Na}$ & - & Element No. 11 - Sodium - Liquid Metal Coolant \\
\hline $\mathrm{Na} / \mathrm{H}_{2} \mathrm{O}$ & - & Sodium/Watex (Reaction) \\
\hline $\mathrm{NaK}$ & - & $\begin{array}{l}\text { A low melting point mixture of Sodium and } \\
\text { Potassium }\end{array}$ \\
\hline NEPA & - & National Environmental Policy Act \\
\hline NNS & - & $\begin{array}{l}\text { American Nuclear Society Non-Nuclear Safety } \\
\text { Class }\end{array}$ \\
\hline $\mathrm{NO}_{\mathbf{x}}$ & - & Oxides of Nitrogen (general) \\
\hline NPDES & - & National Pollution Discharge Elimination System \\
\hline NPGS & - & Nuclear Power (Electric) Generating Station \\
\hline NPSH & - & Net Positive Suction Head \\
\hline NS & - & Nuclear Safety \\
\hline NSPS & - & New Source Performance Standards \\
\hline NSSS & - & Nuclear Steam Supply System \\
\hline NSW & - & Nuclear Service Water (System) \\
\hline OBE & - & Operating Basis Earthquake \\
\hline OP & - & Overpressure \\
\hline $\mathrm{Pb}$ & - & Element No. 82 - Lead \\
\hline PCCW & - & Primary Component Cooling Water (System) \\
\hline
\end{tabular}




\section{3 .3 (Cont'd)}

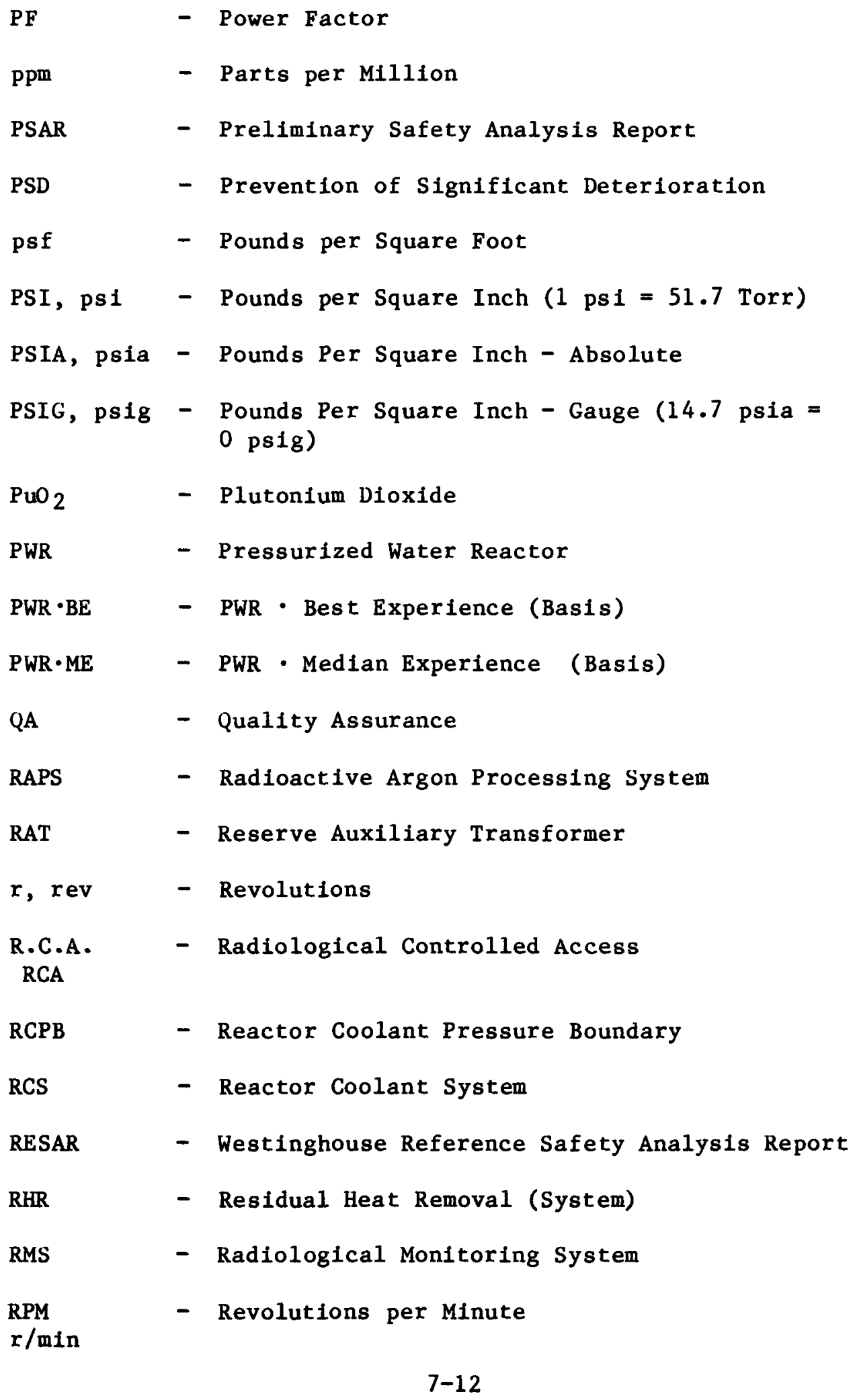




\section{3 .3 (Cont'd)}

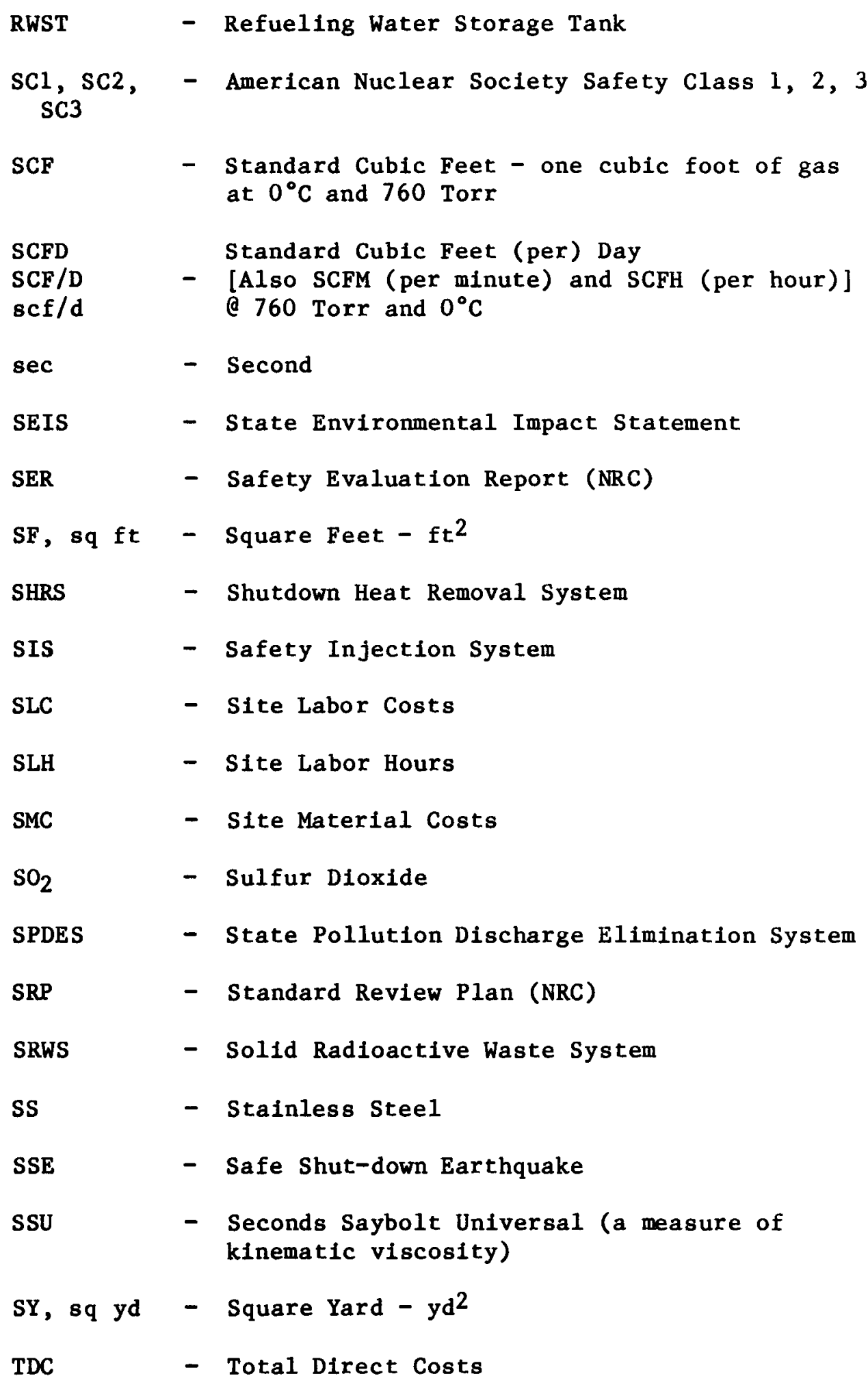


7.3.3 (Cont'd)

$\begin{array}{ll}\text { TDH } & - \text { Total Dynamic Head (discharge head minus } \\ \text { suction head for centrifugal pumps) } \\ \text { T/G } & - \text { Turbine-Generator (Unit) } \\ \text { TIC } & - \text { Total Indirect Costs } \\ \text { TN } & - \text { Ton(s) [2000 pounds }] \\ \text { tph } & - \text { Tons per Hour } \\ \text { tpy } & - \text { Tons per Year } \\ \text { UAT } & - \text { Unit Auxiliary Transformer } \\ \text { UO2 } & - \text { Uranium Dioxide } \\ \text { V } & - \text { Volts } \\ \text { VWO } & - \text { Valves Wide Open } \\ \text { WBS } & - \text { Work Breakdown Structure } \\ \text { We } & - \text { Watts - Electrical } \\ \text { W.g. } & - \text { Water Gage (usually inches of water, } 1 \text { in. w.g. } \\ \text { Wt } & - \text { Watts - Thermal or Weight (depending on context) } \\ \text { yr } & \end{array}$


APPENDIX-A-1

ENERGY ECONOMIC DATA BASE (EEDB) PROGRAM

BIBLIOGRAPHY

POTENTIAL DESIGN FEATURES/PRACTICES FOR NUCLEAR POWER PLANTS OF THE 1990'S AND BEYOND 


\section{APPENDIX A-1}

ENERGY ECONOMIC DATA BASE (EEDB) PROGRAM

BIBLIOGRAPHY OF POTENTIAL DESIGN FEATURES/PRACTICES FOR

NUCLEAR POWER PLANTS OF THE 1990'S AND BEYOND

The following bibliographles provide a limited listing of resources on current activities that may generate data that will be used in the designs of advanced nuclear power plants for the 1990 's and beyond.

Bibliography $I$ is an alphabetized compendium of relevant reports and studies. Bibllography II is a rearrangement of Bibliography I by topic, because many of the citations in Bibliography I cover several subjects. 


\section{A1.1 BIBLIOGRAPHY I (ALPHABETICAL)}

1. .... "A-PWR Aims to Minimize Risks," Nuclear Engineering International, November 1985 , p. 49 .

2. Breeder Reactor Division, Use of Mobile Radioactive Waste Processing Services Cost Reduction Report, Burns \& Roe, Inc., Oradel1, New Jersey: August 1983.

3. Cruickshank, Andrew. "Robots Take a Hand in Inspection, Maintenance and Repair," Nuclear Engineering International, April 1985, pp. 25-39.

4. Iacovino, John. "Advanced PWR Aims at 100 Man-Rems/Year," Nuclear Engineering International (November 1985) pp. 48-50.

5. .... INPO 82-003, Significant Event Evaluation and Information Network (SEE-IN), Program Description, Atlanta: Institute of Nuclear Power Operations, January 1982 .

6. .... INPO 85-010, Good Practice TS-410, Surveillance and Periodic Task Scheduling Program, Atlanta: Institute of Nuclear Power Operations, April 1985.

7. .... INPO 85-013, Good Practice TS-402, Plant Modification Control Program, Atlanta: Institute of Nuclear Power Operations, May 1985.

8. .... INPO 85-014, Good Practice EP-801, Generic Guidance for Emergency Preparedness Review, Atlanta: Institute of Nuclear Power Operations, May 1985.

9. .... INPO 85-016, Good Practice OP-202, Temporary Modification Control, Atlanta: Institute of Nuclear Power Operations, May 1985.

10. .... INPO 85-018, Good Practice OEN-06, Conduct of Practical Exercises During General Employee Training, Atlanta: Institute of Nuclear Power Operations, June 1985 .

11. .... INPO 85-27, An Analysis of Root Causes in 1983 and 1984 Significant Event Reports, Special Event Reports, Atlanta: Institute of Nuclear Power Operations, July 1985.

12. Lapides, Melvin. "Minac - A Small Linear Accelerator with Large Capabilities," Nuclear Engineering International, April 1985, pp. 40-42.

13. Lefkowitz, Sheldon and Parlee, Kenneth A. "Polar Crane Guidelines Evolve from TMI Damage Assessment," Power Engineering, May 1984, pp. 40-43.

14. MacPhee, D. David and Anderson, John. "Retrofit of Feedwater Heaters Reduces Contaminants and Increases Output," Power Engineering, Vo1. 89 No. 4, April 1985, pp. 35-38. 
15. Meininger, Richard D., GEND-050, Three Mile Island Technical Information and Examination Program, Instrumentation and Electrical Summary Report, U.S. Department of Energy, July 1985.

16. Moore, Taylor. "Robots Join the Nuclear Workforce," EPRI Journal, November 1984 , pp. 6-17.

17. Moore, Taylor. "Artificial Intelligence: Human Expertise from Machines," EPRI Journal, June 1985, pp. 6-15.

18. Mosey, David. "Pickering Retubing Keeps Ahead of Schedule," Nuclear Engineering International, April 1985, pp. 21-23.

19. Norgate, Graham. "Artificlal Intelligence: The Future in Nuclear Power Plant Maintenance," Nuclear News, December 1984, pp. 57-61.

20. Office of Nuclear Reactor Regulation, NUREG-0207, Interim Format and Content for a Physical Security Plan for Nuclear Power Plants, U.S. Nuclear Regulatory Commission, February 1977.

21. Operations. "Robotics, EPRI Demo Highlights Various Systems," Nuclear News, December 1984, pp. 50-51.

22. Operations. "Study Compares Japanese, U.S. Plant Maintenance," Nuclear News, Vo1. 28, No. 11, September 1985, pp. 42-50.

23. Payne, Jon. "Japan: Alming For Excellence," Nuclear News, August 1985, pp. $68-75$.

24. Remote Technology Corporation, NUREG/CR-3717, Evaluation of Robotic Inspection Systems at Nuclear Power Plants, U.S. Nuclear Regulatory Commission, March 1984.

25. Rittenhouse, R.C. "Diagnostics for Operation, Maintenance and Upgrading," Power Engineering, March 1985, pp. 30-38.

26. Rittenhouse, R.C. "The Intricacies of Purchasing Power Plant Equipment," Power Engineering, Vol. 89 No. 9, September 1985, pp. 30-38.

27. Smith, Douglas J. "Preventative Maintenance and Cleaning of Major Power Plant Equipment," Power Engineering, Vol. 89, No. 7, July 1985, pp. 42-50.

28. Stone \& Webster Englneering Corporation, CO0-4039-1, Final Report, Plant Systems/ Components Modularization Study, U.S. ERDA, July 1977.

29. Staehr, James P. "A New Approach for Effective Solidification of LLR Waste," Nuclear Plant Safety, Vo1. 2 No. 4, July-August 1984, pp 12-14. 
30. Sutter, Herbert. "The Clean-up of Power Plant Waste Water by Selective Ion Exchange," Nuclear Plant Safety, Vol. 2 No. 4, July-August 1984, pp. 4-8.

31. Tally, Charles W. "Ensuring the Design Lifè of a Nuclear Steam Supply System," Power Engineering, April 1985, pp. 48-50. 


\section{Al.2 BIBLIOGRAPHY II (TOPICAL)}

\section{ALARA}

1. Cruickshank, Andrew. "Robots Take a Hand in Inspection, Maintenance and Repair," Nuclear Engineering International, April 1985, pp. 25-39.

2. Iacovino, John. "Advanced PWR Aims at 100 Man-Rems/Year," Nuclear Engineering International (November 1985) pP. 48-50.

3. Mosey, David. "Pickering Retubing Keeps Ahead of Schedule," Nuclear Engineering International, April 1985, pp. 21-23.

4. Operations. "Robotics, EPRI Demo Highlights Various Systems," Nuclear News, December 1984, pp. 50-51.

5. Remote Technology Corporation, NUREG/CR-3717, Evaluation of Robotic Inspection Systems at Nuclear Power Plants, U.S. Nuclear Regulatory Commission, March 1984 .

\section{Availability}

1. ... "A-PWR Aims to Minimize Risk," Nuclear Engineering International, November 1985 , p. 49.

2. Cruickshank, Andrew. "Robots Take a Hand in Inspection, Maintenance and Repair," Nuclear Engineering International, April 1985, pp. 25-39.

3. Iacovino, John. "Advanced PWR Aims at 100 Man-Rems/Year," Nuclear Engineering International (November 1985) pp. 48-50.

4. Lapides, Melvin. "Minac - A Small Linear Accelerator with Large Capabilities," Nuclear Engineering International, Apri1 1985, pp. 40-42.

5. Lefkowitz, Sheldon and Parlee, Kenneth A. "Polar Crane Guidellnes Evolve from TMI Damage Assessment," Power Engineering, May 1984, pp. 40-43.

6. MacPhee, D. David and Anderson, John. "Retrofit of Feedwater Heaters Reduces Contaminants and Increases Output," Power Engineering, Vol. 89 No. 4, April 1985, pp. 35-38.

7. Meininger; Richard D., GEND-050, Three Mile Island Technical Information and Examination Program, Instrumentation and Electrical Summary Report, U.S. Department of Energy, July 1985 .

8. Moore, Taylor. "Robots Join the Nuclear Workforce," EPRI Journal, November 1984, pp. 6-17. 
9. Moore, Taylor. "Artificial Intelligence: Human Expertise from Machines," EPRI Journal, June 1985, pp. 6-15.

10. Norgate, Graham. "Artificial Intelligence: The Future in Nuclear Power Plant Maintenance," Nuclear News, December 1984, pp. 57-61.

11. Operations. "Robotics, EPRI Demo Highlights Various Systems," Nuclear News, December 1984, pp. 50-51.

12. Operations. "Study Compares Japanese, U.S. Plant Maintenance," Nuclear News, Vol. 28, No. 11, September 1985, pp. 42-50.

13. Payne, Jon. "Japan: Aiming For Excellence," Nuclear News, August 1985, pp. 68-75.

14. Remote Technology Corporation, NUREG/CR-3717, Evaluation of Robotic Inspection Systems at Nuclear Power Plants, U.S. Nuclear Regulatory Commission, March 1984.

15. Rittenhouse, R.C. "Diagnostics for Operation, Maintenance and Upgrading," Power Engineering, March 1985, pp. 30-38.

16. Tally, Charles W. "Ensuring the Design Life of a Nuclear Steam Supply System," Power Engineering, April 1985, pp. 48-50.

\section{Computer}

1. Rittenhouse, R.C. "Diagnostics for Operation, Maintenance and Upgrading," Power Engineering, March 1985, pp. 30-38.

2. Smith, Douglas J. "Preventative Maintenance and Cleaning of Major Power Plant Equipment," Power Engineering, Vol. 89, No. 7, July 1985, pp. 42-50.

\section{Equipment Qualification}

1. Lefkowitz, Sheldon and Parlee, Kenneth A. "Polar Crane Guidelines Evolve from TMI Damage Assessment," Power Engineering, May 1984, pp. 40-43.

2. MacPhee, D. David and Anderson, John. "Retrofit of Feedwater Heaters Reduces Contaminants and Increases Output," Power Engineering, Vol. 89 No. 4, April 1985, pp. 35-38.

3. Meininger, Richard D., GEND-050, Three Mile Island Technical Information and Examination Program, Instrumentation and Electrical Summary Report, U.S. Department of Energy, July 1985. 
4. Payne, Jon. "Japan: Aiming For Excellence," Nuclear News, August 1985, pp. $68-75$.

5. Rittenhouse, R.C. "Diagnostics for Operation, Maintenance and Upgrading," Power Engineering, March 1985, pp. 30-38.

\section{Maintenance Activities}

1. Cruickshank, Andrew. "Robots Take a Hand in Inspection, Maintenance and Repair," Nuclear Engineering International, Apri1 1985, pp. 25-39.

2. Lapides, Melvin. "Minac - A Sma11 Linear Accelerator with Large Capabil1ties," Nuclear Engineering International, April 1985, pp. 40-42.

3. Lefkowitz, Sheldon and Parlee, Kenneth A. "Polar Crane Guidelines Evolve from TMI Damage Assessment," Power Engineering, May 1984, pp. 40-43.

4. Moore, Taylor. "Robots Join the Nuclear Workforce," EPRI Journal, November 1984 , pp. 6-17.

5. Moore, Taylor. "Artificial Intelligence: Human Expertise from Machines," EPRI Journal, June 1985, pp. 6-15.

6. Mosey, David. "Pickering Retubing Keeps Ahead of Schedule," Nuclear Eng1neering International, Apri1 1985, pp. 21-23.

7. Norgate, Graham. "Artificial Intelligence: The Future in Nuclear Power Plant Maintenance," Nuclear News, December 1984, pp. 57-61.

8. Operations. "Robotics, EPRI Demo Highlights Various Systems," Nuclear News, December 1984, pp. 50-51.

9. Operations. "Study Compares Japanese, U.S. Plant Maintenance," Nuclear News, Vol. 28, No. 11, September 1985, pp. 42-50.

10. Remote Technology Corporation, NUREG/CR-3717, Evaluation of Robotic Inspection Systems at Nuclear Power Plants, U.S. Nuclear Regulatory Commission, March 1984 .

11. Rittenhouse, R.C. "Diagnostics for Operation, Maintenance and Upgrading," Power Engineering, March 1985, pp. 30-38.

12. Smith, Douglas J. "Preventative Maintenance and Cleaning of Major Power Plant Equipment," Power Engineering, Vol. 89, No. 7, July 1985, pp. 42-50. 


\section{Modularization}

1. Payne, Jon. "Japan: Alming For Excellence," Nuclear News, August 1985, pp. 68-75.

2. Stone \& Webster Engineering Corporation, C00-4039-1, Final Report, Plant Systems/Components Modularization Study, U.S. ERDA, Ju1y 1977.

\section{Operations and Operability}

1. .... "A-PWR Alms to Minimize Risks," Nuclear Engineering International, November 1985, p. 49.

2. Crulckshank, Andrew. "Robots Take a Hand in Inspection, MaIntenance and Repair," Nuclear Engineering International, April 1985, pp. 25-39.

3. Iacovino, John. "Advanced PWR Aims at 100 Man-Rems/Year," Nuclear Eng1neering International (November 1985) pp. 48-50.

4. Lapides, Melvin. "Minac - A Small Linear Accelerator with Large Capabilities," Nuclear Engineering International, Apri1 1985, pp. 40-42.

5. Lefkowitz, Sheldon and Parlee, Kenneth A. "Polar Crane Guidelines Evolve from TMI Damage Assessment," Power Engineering, May 1984, pp. 40-43.

6. Moore, Taylor. "Robots Joln the Nuclear Workforce," EPRI Journal, November 1984, pp. 6-17.

7. Moore, Taylor. "Artificial Intelligence: Human Expertise from Machines," EPRI Journal, June 1985, pp. 6-15.

8. Norgate, Graham. "Artificial Intelligence: The Future in Nuclear Power Plant Maintenance," Nuclear News, December 1984, pp. 57-61.

9. Office of Nuclear Reactor Regulation, NUREG-0207, Interim Format and Content for a Physical Security Plan for Nuclear Power Plants, U.S. Nuclear Regulatory Commission, February 1977.

10. Operations. "Robotics, EPRI Demo Highlights Various Systems," Nuclear News, December 1984, pp. 50-51.

11. Payne, Jon. "Japan: Alming For Excellence," Nuclear News, August 1985, pp. 68-75.

12. Remote Technology Corporation, NUREG/CR-3717, Evaluation of Robotic Inspection Systems at Nuclear Power Plants, U.S. Nuclear Regulatory Commission, March 1984. 
13. Rittenhouse, R.C. "Diagnostics for Operation, Maintenance and Upgrading," Power Engineering, March 1985, pp. 30-38.

14. Tally, Charles W. "Ensuring the Design Life of a Nuclear Steam Supply System," Power Engineering, April 1985, pp. 48-50.

\section{Radioactive Waste Disposal}

1. Breeder Reactor Division, Use of Mobile Radioactive Waste Processing Services Cost Reduction Report, Burns \& Roe, Inc., Orade11, New Jersey: August 1983.

\section{$\underline{\text { Robotics and Artificial Intelligence }}$}

1. Cruickshank, Andrew. "Robots Take a Hand in Inspection, Maintenance and Repair," Nuclear Engineering International, Apri1 1985, pp. 25-39.

2. Moore, Taylor. "Robots Join the Nuclear Workforce," EPRI Journal, November 1984, pp. 6-17.

3. Moore, Taylor. "Artificial Intelligence: Human Expertise from Machines," EPRI Journal, June 1985, pp. 6-15.

4. Norgate, Graham. "Artificial Intelligence: The Future in Nuclear Power Plant Maintenance," Nuclear News, December 1984, pp. 57-61.

5. Operations. "Robotics, EPRI Demo Highlights Various Systems," Nuclear News, December 1984, pp. 50-51.

6. Payne, Jon. "Japan: Aiming For Excellence," Nuclear News, August 1985, PP. 68-75.

7. Remote Technology Corporation, NUREG/CR-3717, Evaluation of Robotic Inspection Systems at Nuclear Power Plants, U.S. Nuclear Regulatory Commission, March 1984.

8. Stone \& Webster Engineering Corporation, C00-4039-1, Final Report, Plant Systems/Components Modularization Study, U.S. ERDA, July 1977 .

\section{Steam Generators and Turbine Generators}

1. .... "A-PWR Aims to Minimize Risks," Nuclear Engineering Internationa1, November 1985 , p. 49.

2. Cruickshank, Andrew. "Robots Take a Hand in Inspection, Maintenance and Repair," Nuclear Engineering International, April 1985, pp. 25-39. 
3. Iacovino, John. "Advanced PWR Aims at 100 Man-Rems/Year," Nuclear Engineering International, November 1985, pp. 48-50.

4. MacPhee, D. David and Anderson, John. "Retrofit of Feedwater Heaters Reduces Contaminants and Increases Output," Power Engineering, Vol. 89 No. 4, April 1985, pp. 35-38.

5. Payne, Jon. "Japan: Aiming For Excellence," Nuclear News, August 1985, pp. 68-75.

6. Smith, Douglas J. "Preventative Maintenance and Cleaning of Major Power Plant Equipment," Power Engineering, Vol. 89, No. 7, July 1985, pp. 42-50.

7. Tally, Charles W. "Ensuring the Design Life of a Nuclear Steam Supply System," Power Englneering, April 1985, pp. 48-50. 